\title{
COMPACTIFICATIONS OF PEL-TYPE SHIMURA VARIETIES IN RAMIFIED CHARACTERISTICS
}

\author{
KAI-WEN LAN \\ University of Minnesota, Minneapolis, MN 55455, USA; \\ email: kwlan@math.umn.edu
}

Received 14 October 2014; accepted 5 December 2015

\begin{abstract}
We show that, by taking normalizations over certain auxiliary good reduction integral models, one obtains integral models of toroidal and minimal compactifications of PEL-type Shimura varieties which enjoy many features of the good reduction theory studied as in the earlier works of Faltings and Chai's and the author's. We treat all PEL-type cases uniformly, with no assumption on the level, ramifications, and residue characteristics involved.
\end{abstract}

2010 Mathematics Subject Classification: 11G18 (primary); 11G15, 14D06 (secondary)

\section{Introduction}

In recent years, we have witnessed a rapid development in the arithmetic applications of noncompact Shimura varieties, in which the integral models of toroidal and minimal compactifications have played important roles. So far, such applications have almost always assumed that there are some bottom levels at which the integral models have good reductions, which is the case when the residue characteristics are unramified in all linear algebraic data involved, so that the Shimura varieties in question have smooth integral models which can be constructed and compactified using the theories of deformation and degeneration as in [10] and [30].

Nevertheless, as remarked in the introduction of [31], since the theory of degeneration developed in [42], [10], and [30] works as long as the generic characteristic is good (and as long as the base of degenerations are noetherian

(c) The Author 2016. This is an Open Access article, distributed under the terms of the Creative Commons Attribution licence (http://creativecommons.org/licenses/by/4.0/), which permits unrestricted re-use, distribution, and reproduction in any medium, provided the original work is properly cited. 
normal), there is, a priori, no reason that we cannot consider integral models of Shimura varieties and their compactifications with bad reductions. Also, recent breakthroughs in the theory of local models have shown that, even when allowing rather deep ramifications, it is not so unreasonable to consider integral models of Shimura varieties defined by taking normalizations of the (schematic) closures of the images of characteristic zero Shimura varieties in certain auxiliary good reduction integral models (for simplicity, we shall just say that such integral models are constructed 'by taking normalizations of certain auxiliary good reduction integral models', or just 'by normalization'). This is because a large class of useful models defined by representing moduli problems can be shown to be normal, or close to being so, in the sense that the closures of their characteristic zero fibers are normal. Since the theory of degeneration works well over noetherian normal base schemes, the time seems ripe for systematically studying the construction of integral models of compactifications by normalization.

Let us be more precise about the integral models we will consider (without explicit definitions, to be given later in the main text). Consider any PEL moduli problem $\mathrm{M}_{\mathcal{H}}$ over $F_{0}$ (the reflex field of the PEL datum defining $\mathrm{M}_{\mathcal{H}}$ ), as in [30, Section 1.4.1] (with $\square=\emptyset$ there), parameterizing tuples $\left(A, \lambda, i, \alpha_{\mathcal{H}}\right)$, where $A$ is an abelian scheme over some base scheme, $\lambda: A \rightarrow A^{\vee}$ is a polarization, $i$ is an endomorphism structure, and $\alpha_{\mathcal{H}}$ is a level- $\mathcal{H}$ structure. Here $\mathcal{H}$ is an open compact subgroup of $\mathrm{G}(\hat{\mathbb{Z}})$, where the group functor $\mathrm{G}$ over $\operatorname{Spec}(\mathbb{Z})$ is defined as in [30, Definition 1.2.1.6]. Let $p>0$ be any rational prime number. For technical simplicity, let us assume that the image $\mathcal{H}^{p}$ of $\mathcal{H}$ under the canonical homomorphism $\mathrm{G}(\hat{\mathbb{Z}}) \rightarrow \mathrm{G}\left(\hat{\mathbb{Z}}^{p}\right)$ is neat. Consider a collection of auxiliary moduli problems $\left\{\mathrm{M}_{\mathcal{H}_{\mathrm{j}, \text { aux }}}\right\}_{\mathrm{j} \in \mathrm{J}}$, where each $\mathrm{M}_{\mathcal{H}_{\mathrm{j} \text {,aux }}}$ is a $\operatorname{good}$ reduction moduli problem over $\mathcal{O}_{F_{0, \mathrm{j}, \text { aux }},(p)}$ (the ring of $p$-integers in the reflex field $F_{0, \text { j,aux }}$ of the PEL datum defining $\mathrm{M}_{\mathcal{H}_{\mathrm{j}, \text { aux }}}$ ), defined as in [30, Section 1.4.1] (with $\square=\{p\}$ there), parameterizing tuples $\left(A_{\mathrm{j}, \text { aux }}, \lambda_{\mathrm{j}, \text { aux }}, i_{\mathrm{j}, \text { aux }}, \alpha_{\mathcal{H}_{\mathrm{j}, \text { aux }}}\right)$, with morphisms $\mathrm{M}_{\mathcal{H}} \rightarrow \mathrm{M}_{\mathcal{H}_{\mathrm{j}, \text { aux }}} \otimes_{\mathbb{Z}} \mathbb{Q}$ between moduli problems defined by assigning $\left(A_{\mathrm{j}, \text { aux }}\right.$, $\left.\lambda_{\mathrm{j}, \text { aux }}, i_{\mathrm{j} \text {,aux }}, \alpha_{\mathcal{H}_{\mathrm{j} \text {,ax }}}\right)$ to $\left(A, \lambda, i, \alpha_{\mathcal{H}}\right)$ (in a way that will be made explicit), where $\left(A_{\mathrm{j}, \text { aux }}, \lambda_{\mathrm{j} \text {,aux }}\right)$ is a prime-to- $p$ polarized abelian scheme defined by $(A, \lambda)$, using Zarhin's trick when $\lambda$ is not prime to $p$, where $i_{\text {aux }}$ is the restriction of $i$ to a subalgebra unramified at $p$, and where $\alpha_{\mathcal{H}_{\mathrm{j} \text {,aux }}}$ is a (possibly lower) level structure away from $p$ induced by $\alpha_{\mathcal{H}}$ (up to Hecke twist). Then we construct a $p$-integral model $\overrightarrow{\mathrm{M}}_{\mathcal{H}, \mathrm{J}}$ of $\mathrm{M}_{\mathcal{H}}$ as the normalization of $\prod_{\mathrm{j} \in \mathrm{J}} \mathrm{M}_{\mathcal{H}_{\mathrm{j}, \text { aux }}}$ under the canonical morphism $\mathrm{M}_{\mathcal{H}} \rightarrow \prod_{\mathrm{j} \in \mathrm{J}} \mathrm{M}_{\mathcal{H}_{\mathrm{j}, \text { aux }}}$ (the use of a collection $\left\{\mathrm{M}_{\mathcal{H}_{\mathrm{j}, \text { aux }}}\right\}_{\mathrm{j} \in \mathrm{J}}$ takes care of the consideration of multichains of isogenies as in [51, Ch. 3], or of more general collections of quasiisogenous polarized abelian schemes). We similarly 
define $p$-integral models of toroidal and minimal compactifications of $\mathrm{M}_{\mathcal{H}}$ using the toroidal and minimal compactifications of the auxiliary moduli problems constructed as in [30, Chs. 6 and 7]. For simplicity, we shall call these integral models rather than $p$-integral models (we obtain models over the whole integer ring, or any localizations of it, by similar considerations).

For example, if $\mathrm{M}_{\mathcal{H}}$ is the modular curve (over $\mathbb{Q}$ ) of principal level $n$, where $n=n_{0} p^{r}$ for some integer $n_{0} \geqslant 3$ prime to $p$ and for some integer $r \geqslant 0$, then we can take $\mathrm{M}_{\mathrm{j}_{0} \text {,aux }}$ (where $\mathrm{J}$ is a singleton $\left\{\mathrm{j}_{0}\right\}$ ) to be the modular curve (over $\mathbb{Z}_{(p)}$ ) of principal level $m$ for any integer $m \geqslant 3$ dividing $n_{0}$ (this is essentially the same approach taken in [9, III, Definition 3.3]). For another example, if $\mathrm{M}_{\mathcal{H}}$ is the Siegel moduli (over $\mathbb{Q}$ ) of genus $g$, degree $d_{\mathrm{j}}^{2}$ (possibly divisible by $p$ ), and principal level $n$ for some integer $n=n_{0} p^{r}$ as above, then we can take $\mathrm{M}_{\mathrm{j}_{0} \text { aux }}$ (where $\mathrm{J}$ is a singleton $\left\{\mathrm{j}_{0}\right\}$ ) to be the principally polarized Siegel moduli (over $\mathbb{Z}_{(p)}$ ) of genus $8 g$ and principal level $m$, for any integer $m \geqslant 3$ dividing $n_{0}$. For yet another example, if $\mathrm{M}_{\mathcal{H}}$ is a unitary Shimura variety with endomorphisms by a maximal order of a $\mathrm{CM}$ field totally ramified at $p$, and with Iwahori level structures (at $p$ ) realized by chains of ( $p$-power-degree) isogenies $A=A_{0} \rightarrow A_{1} \rightarrow \cdots \rightarrow A_{m}=A$ of abelian schemes with compatible additional structures, then we can take $\mathrm{J}$ to be any subset of $\{0,1, \ldots, m\}$, and $\left\{\mathrm{M}_{\mathrm{j}, \text { aux }}\right\}_{\mathrm{j} \in \mathrm{J}}$ to be a collection of principally polarized Siegel moduli, with the morphism $\mathrm{M}_{\mathcal{H}} \rightarrow \mathrm{M}_{\mathrm{j}, \text { aux }}$ given by applying Zarhin's trick to $A_{\mathrm{j}}$ (and its polarization), by forgetting the endomorphism structure, and by retaining only the level structure away from $p$, for each $\mathrm{j} \in \mathrm{J}$. Different choices of the subset $\mathrm{J}$ generally define different $\overrightarrow{\mathrm{M}}_{\mathcal{H}, \mathrm{J}}$ 's by normalization (it might be helpful to take a quick look at Examples 2.3, 2.4, 13.11, and 13.12, without studying them in detail).

This article aims at showing that many features of the good reduction theory as in [30] extend to the integral models of toroidal and minimal compactifications constructed by normalization, despite the fact that the constructions (as explained above) are rather crude. We will justify the folklore belief that 'the toroidal boundary should be no more singular than the interior', without studying the interior.

We will show that, by taking normalizations over certain auxiliary good reduction integral models of toroidal compactifications (as above), we obtain integral models of toroidal compactifications associated with certain compatible collections of (possibly nonsmooth) induced cone decompositions, whose local properties in terms of geometric normality of fibers and Cohen-Macaulayness are nevertheless as nice as the integral models of Shimura varieties defined by normalization. Moreover, these integral models of toroidal and minimal compactifications admit boundary stratifications analogous to the ones in good characteristics (including zero), and the completions of the integral models of 
toroidal compactifications along their strata can be explicitly compared with the completions of certain putative boundary charts parameterizing degeneration data of PEL structures. These assertions will be proved with essentially no assumption on the integral PEL data defining the moduli problems (we will only need [30, Condition 1.4.3.10] to hold, which can always be achieved by slightly modifying the choices of integral PEL data).

As examples of applications of our results, we will combine our results with related results in the theory of local models in the ramified case, and show that certain integral models of toroidal and minimal compactifications have the same number of geometric irreducible components in their generic and special fibers. We also show the density of ordinary loci in certain deeply ramified cases, by combining the above with the technique for showing nonemptiness as in [31, Section 6.3.3].

Here is an outline of the article.

In Section 2, we review the basic setting for the definition of our PEL-type moduli problems in characteristic zero, which will be called PEL-type Shimura varieties for the sake of simplicity, despite the well-known issue of the failure of Hasse's principle (this is harmless because the canonical models as in [18] and [49], which are based on the construction in [2], are open and closed in the complex fibers of our models; see [28]). For the sake of completeness, we also consider collections of lattices twisted by group actions, which define moduli problems for collections of abelian schemes with PEL structures related to each other by $\mathbb{Q}^{\times}$-isogenies (that is, quasiisogenies; see [30, Definitions 1.3.1.16 and 1.3.1.17]) (our theory applies, in particular, to the parahoric setting in [51] and in later works built on it).

In Section 3, we explain how the association of degeneration data behaves under $\mathbb{Q}^{\times}$-isogenies defined by the collections of lattices introduced in Section 2. The assertions in this section are perhaps unsurprising, but the explanations for them are quite elaborate. Since the technical difficulties in this section are rather different from those in later sections, we suggest that first-time readers skip this section.

In Sections 4 and 5, we introduce certain auxiliary choices of good reduction integral models of PEL-type Shimura varieties, together with their toroidal and minimal compactifications. In Section 6, we define integral models of the Shimura varieties in question, together with their minimal compactifications, by taking normalizations of (products of) such auxiliary good reduction integral models (Propositions 6.1 and 6.4). For the integral models of Shimura varieties thus defined, we can easily show that they are independent of the auxiliary choices in Sections 4 and 5. However, for the integral models of the minimal compactifications, our argument is rather indirect, and we will have to wait until 
many other results are proved; see Section 12 below. The materials in these three sections follow closely those in [31, Sections 2.1.1, 2.1.2, and 2.2.1], except that we have to consider auxiliary choices compatibly associated with the collections of lattices.

In Section 7, we define certain toroidal compactifications of the integral models of Shimura varieties, with compatible collections of cone decompositions induced by those of the auxiliary toroidal compactifications, and show that they satisfy certain universal property generalizing the one in [30, Theorem 6.4.1.1(6)] (see Theorem 7.14). Such a universal property is the foundation for all our later arguments.

In Section 8, we construct putative boundary charts, and show that certain formal schemes defined by them admit canonical morphisms to the toroidal compactifications defined by normalization. In Section 9, we show that the images of the underlying topological maps of such morphisms are locally closed and define stratifications of the toroidal compactifications defined by normalization, with properties as in characteristic zero as in [30, Theorem 6.4.1.1(2)] (see Theorem 9.13). In Section 10, we show that the canonical morphisms from the formal schemes defined by putative boundary charts to the toroidal compactifications defined by normalization induce isomorphisms from the former to the formal completions of the latter along the (locally closed) image strata (see Theorem 10.13). Such isomorphisms will play important roles in subsequent sections. It follows that the special fibers of integral models defined by normalization are dense in the corresponding special fibers of toroidal compactifications defined by normalization (see Corollary 10.18).

In Section 11, we show that the tautological objects over our moduli problems in characteristic zero, which are collections of abelian schemes with PEL structures related to each other by $\mathbb{Q}^{\times}$-isogenies, uniquely extends to collections of semiabelian schemes equipped with similar structures over the toroidal compactifications in mixed characteristics defined by normalization (see Theorem 11.2). We also improve the universal property in Theorem 7.14 and show that the toroidal compactifications defined by normalization are independent of the auxiliary choices in Sections 4 and 5 (see Theorem 11.4 and Corollary 11.7).

In Section 12, we show that the minimal compactifications defined by normalization admit stratifications with properties as in characteristic zero as in [30, Theorem 7.2.4.1 (4) and (5)] (see Theorems 12.1 and 12.16). It follows that the special fibers of integral models defined by normalization are dense in the corresponding special fibers of minimal compactifications defined by normalization (see Corollary 12.5 and Remark 12.6). Consequently, we can finally show that the integral models of minimal compactifications defined by 
normalization are also independent of the auxiliary choices in Sections 4 and 5 (see Corollary 12.7).

In Section 13, we study the morphisms induced functorially by varying the levels, collections of lattices, and cone decompositions, and work out some examples. In particular, we obtain morphisms extending the ones in characteristic zero defining Hecke correspondences among Shimura varieties (see Proposition 13.15).

In Section 14, we show that the local properties of the toroidal compactifications defined by normalization are as nice as the integral models of Shimura varieties themselves, when it comes to the geometric normality of fibers and Cohen-Macaulayness (and also the regularity and geometric regularity of fibers when the cone decompositions are smooth) (see Propositions 14.1 and 14.2; see also Corollary 14.4 for some well-known application). We also work out some examples, based on the theory of local models, where such results apply (see Lemmas 14.6 and 14.7). In Section 15, we show the density of ordinary loci in some of such examples (see Proposition 15.1 and Corollary 15.2).

In Section 16, we conclude the article with some remarks comparing the results in this article with other known results, including our own earlier ones.

The arguments in this article will be built on the theory developed in [30], and some familiarity with the theory there will be necessary. The readers may find the summaries, explanations, and reformulations in [29, Section 1] and [31, Sections 1.1, 1.2, 1.3.1, and 1.3.2] helpful. The notation system in this article is probably more complicated than it absolutely has to be, but we have chosen to make it consistent with most of those in [30] and [31] (and other works dependent on them), so that readers already familiar with them will not have to learn a completely new notation system. We will make it clear when we occasionally do introduce some simplifications.

We shall follow [30, Notation and Conventions] unless otherwise specified. While for practical reasons we are unable to review all notions we have inherited from [30] and [31], we recommend that the readers make use of the reasonably detailed indices and tables of contents of these works when looking for the numerous definitions.

\section{Basic setting}

Suppose we have an integral PEL datum $\left(\mathcal{O}, \star, L,\langle\cdot, \cdot\rangle, h_{0}\right)$, where $\mathcal{O}$ is an order in a semisimple algebra finite-dimensional over $\mathbb{Q}$, together with a positive involution ${ }^{\star}$, and where $\left(L,\langle\cdot, \cdot\rangle, h_{0}\right)$ is a PEL-type $\mathcal{O}$-lattice as in [30, Definition 1.2.1.3], where $\langle\cdot, \cdot\rangle: L \times L \rightarrow \mathbb{Z}(1)$ is an alternating pairing satisfying $\langle b x, y\rangle=$ $\left\langle x, b^{\star} y\right\rangle$ for any $x, y \in L$ and $b \in \mathcal{O}$, together with an $\mathbb{R}$-algebra homomorphism 
$h_{0}: \mathbb{C} \rightarrow \operatorname{End}_{\mathcal{O} \otimes_{\mathbb{R}} \mathbb{R}}\left(L \otimes_{\mathbb{Z}} \mathbb{R}\right)$ satisfying $\left\langle h_{0}(z) x, y\right\rangle=\left\langle x, h_{0}\left(z^{c}\right) y\right\rangle$ for any $x$, $y \in L$ and $z \in \mathbb{C}$, and satisfying $(2 \pi \sqrt{-1})^{-1}\left\langle x, h_{0}(\sqrt{-1}) x\right\rangle>0$ for any nonzero $x \in L$ (in [30, Definition 1.2.1.3] $h_{0}$ was denoted by $h$ ). Such a tuple $(\mathcal{O}, \star, L$, $\left.\langle\cdot, \cdot\rangle, h_{0}\right)$ is an integral version of the PEL datum $\left(B, \star, V,\langle\cdot, \cdot\rangle, h_{0}\right)$ in [26] and related works.

The datum of $\left(\mathcal{O}, \star, L,\langle\cdot, \cdot\rangle, h_{0}\right)$ defines a group functor $\mathrm{G}$ over $\operatorname{Spec}(\mathbb{Z})$ (as in [30, Definition 1.2.1.6]), and defines the reflex field $F_{0}$ (as in [30, Definition 1.2.5.4]). In what follows, we will allow $F_{0}$ to be any finite extension field of the reflex field (the theory works for any such extension field).

Let $\mathcal{H}$ be an open compact subgroup of $\mathrm{G}(\hat{\mathbb{Z}})$. By [30, Definition 1.4.1.4] (with $=\emptyset$ there $)$, the data of $\left(L,\langle\cdot, \cdot\rangle, h_{0}\right)$ and $\mathcal{H}$ define a moduli problem $\mathrm{M}_{\mathcal{H}}$ over $\mathrm{S}_{0}=\operatorname{Spec}\left(F_{0}\right)$, parameterizing tuples $\left(A, \lambda, i, \alpha_{\mathcal{H}}\right)$ over schemes $S$ over $\mathrm{S}_{0}$, where:

(1) $A \rightarrow S$ is an abelian scheme;

(2) $\lambda: A \rightarrow A^{\vee}$ is a polarization;

(3) $i: \mathcal{O} \hookrightarrow \operatorname{End}_{S}(A)$ is an $\mathcal{O}$-endomorphism structure as in [30, Definition 1.3.3.1];

(4) $\underline{\mathrm{Lie}}_{A / S}$ with its $\mathcal{O} \otimes_{\mathbb{Z}} \mathbb{Q}$-module structure given by $i$ satisfies the determinantal condition in [30, Definition 1.3.4.1] given by $\left(L \otimes_{\mathbb{Z}} \mathbb{R},\langle\cdot, \cdot\rangle, h_{0}\right)$; and

(5) $\alpha_{\mathcal{H}}$ is an (integral) level- $\mathcal{H}$ structure of $(A, \lambda, i)$ of type $\left(L \otimes_{\mathbb{Z}} \hat{\mathbb{Z}},\langle\cdot, \cdot\rangle\right)$ as in [30, Definition 1.3.7.6].

By [30, Theorem 1.4.1.11 and Corollary 7.2.3.10], $\mathrm{M}_{\mathcal{H}}$ is an algebraic stack separated, smooth, and of finite type over $\mathrm{S}_{0}$, which is representable by a scheme quasiprojective (and smooth) over $\mathrm{S}_{0}$ when $\mathcal{H}$ is neat (see [49, Section 0.6] or [30, Definition 1.4.1.8] for the definition of neatness).

Given the above $\left(\mathcal{O}, \star, L,\langle\cdot, \cdot\rangle, h_{0}\right)$ and $\mathcal{H} \subset \mathrm{G}(\hat{\mathbb{Z}})$, suppose moreover that we have a nonempty collection $\left\{\left(g_{\mathrm{j}}, L_{\mathrm{j}},\langle\cdot, \cdot\rangle_{\mathrm{j}}\right)\right\}_{\mathrm{j} \in \mathrm{J}}$, where for each $\mathrm{j} \in \mathrm{J}$ the triple $\left(g_{\mathrm{j}}, L_{\mathrm{j}},\langle\cdot, \cdot\rangle_{\mathrm{j}}\right)$ consists of the following data.

(1) $g_{\mathrm{j}} \in \mathrm{G}\left(\mathbb{A}^{\infty}\right)$;

(2) $L_{\mathrm{j}} \subset L \otimes_{\mathbb{Z}} \mathbb{Q}$ is a $\mathcal{O}$-lattice;

(3) $\langle\cdot, \cdot\rangle_{\mathrm{j}}: L_{\mathrm{j}} \times L_{\mathrm{j}} \rightarrow \mathbb{Z}(1)$ is an alternating pairing such that $\langle\cdot, \cdot\rangle_{\mathrm{j}} \otimes_{\mathbb{Z}} \mathbb{Q}$ is a $\mathbb{Q}_{>0}^{\times}$-multiple of $\langle\cdot, \cdot\rangle \otimes_{\mathbb{Z}} \mathbb{Q}$ (when both are viewed as $\mathbb{Q}(1)$-valued pairings on $L \otimes_{\mathbb{Z}} \mathbb{Q}$ ), which defines a group functor $\mathrm{G}_{\mathrm{j}}$ over $\operatorname{Spec}(\mathbb{Z})$ (as in [30, Definition 1.2.1.6]), equipped with a canonical isomorphism

$$
\mathrm{G}_{\mathrm{j}} \underset{\mathbb{Z}}{\otimes} \mathbb{Q} \cong \underset{\mathbb{Z}}{\otimes} \underset{\mathbb{Q}}{\mathrm{Q}}
$$


(4) by also viewing $h_{0}$ as a polarization of $\left(L_{\mathrm{j}},\langle\cdot, \cdot\rangle_{\mathrm{j}}, \mathbb{Z}(1)\right)$, as in [30, Definition 1.2.1.2], $\left(L_{\mathrm{j}},\langle\cdot, \cdot\rangle_{\mathrm{j}}, h_{0}\right)$ is also a PEL-type $\mathcal{O}$-lattice as in [30, Definition 1.2.1.3]; and

(5) let us denote by $\mathcal{H}_{\mathrm{j}}$ the preimage of $g_{\mathrm{j}}^{-1} \mathcal{H} g_{\mathrm{j}}$ under the canonical isomorphism $\mathrm{G}_{\mathrm{j}}\left(\mathbb{A}^{\infty}\right) \cong \mathrm{G}\left(\mathbb{A}^{\infty}\right)$. Then we require that $\mathcal{H}_{\mathrm{j}} \subset \mathrm{G}_{\mathrm{j}}(\hat{\mathbb{Z}})$, or equivalently that the action of $g_{\mathrm{j}}^{-1} \mathcal{H} g_{\mathrm{j}}$ stabilizes the submodule $L_{\mathrm{j}} \otimes_{\mathbb{Z}} \hat{\mathbb{Z}}$ of $L \otimes_{\mathbb{Z}} \mathbb{A}^{\infty}$.

Moreover, as in [30, Condition 1.4.3.10], we shall assume that there exists some maximal order $\mathcal{O}^{\prime}$ in $\mathcal{O} \otimes_{\mathbb{Z}} \mathbb{Q}$ containing $\mathcal{O}$ such that, for every $\mathrm{j} \in \mathrm{J}$, the action of $\mathcal{O}$ on $L_{\mathrm{j}}$ extends to an action of $\mathcal{O}^{\prime}$.

For each $\mathrm{j} \in \mathrm{J}$, we have a moduli problem $\mathrm{M}_{\mathcal{H}_{\mathrm{j}}}$ defined by the integral PEL datum $\left(\mathcal{O}, \star, L_{\mathrm{j}},\langle\cdot, \cdot\rangle_{\mathrm{j}}, h_{0}\right)$ as in [30, Definition 1.4.1.4] (with $\square=\emptyset$ there), parameterizing tuples $\left(A_{\mathrm{j}}, \lambda_{\mathrm{j}}, i_{\mathrm{j}}, \alpha_{\mathcal{H}_{\mathrm{j}}}\right)$ over schemes $S$ over $\mathrm{S}_{0}$ as above (but with $\left(L \otimes_{\mathbb{Z}} \hat{\mathbb{Z}},\langle\cdot, \cdot\rangle\right)$ replaced with $\left(L_{\mathrm{j}} \otimes_{\mathbb{Z}} \hat{\mathbb{Z}},\langle\cdot, \cdot\rangle_{\mathrm{j}}\right)$ in the definition of level- $\mathcal{H}_{\mathrm{j}}$ structures), and where

$$
\mathrm{M}_{\mathcal{H}} \stackrel{\sim}{\rightarrow} \mathrm{M}_{\mathcal{H}_{\mathrm{j}}}
$$

is a canonical isomorphism given by [30, Proposition 1.4.3.4 and Corollary 1.4.3.8], realized by sending objects parameterized by $\mathrm{M}_{\mathcal{H}}$ to their isogeny twists (a special case of this will be spelled out in Section 3).

REMARK 2.2. While $\mathbf{M}_{\mathcal{H}}$ and $\mathrm{M}_{\mathcal{H}_{\mathrm{j}}}$ are canonically isomorphic to each other, their tautological abelian schemes differ by a $\mathbb{Q}^{\times}$-isogeny, which is generally not a $\mathbb{Z}_{(p)}^{\times}$isogeny. We will see in the next few sections that different $\mathrm{M}_{\mathcal{H}_{\mathrm{j}}}$ 's are associated with naturally different auxiliary integral models.

EXAMPLE 2.3 (Simplest case). Suppose $\mathrm{J}=\left\{\mathrm{j}_{0}\right\}$ is a singleton, with the simplest choice $\left(g_{\mathrm{j}_{0}}, L_{\mathrm{j}_{0}},\langle\cdot, \cdot\rangle_{\mathrm{j}_{0}}\right)=(1, L,\langle\cdot, \cdot\rangle)$. In this case, we will study (mixed characteristics) degenerations of objects parameterized by $M_{\mathcal{H}}$.

EXAMPLE 2.4 (Parahoric levels at $p$ ). Suppose that $p>0$ is a rational prime number, that $\mathcal{O} \otimes_{\mathbb{Z}} \mathbb{Q}_{p}$ is simple, and that $\mathcal{O} \otimes_{\mathbb{Z}} \mathbb{Z}_{p}$ is a maximal order in $\mathcal{O} \otimes_{\mathbb{Z}} \mathbb{Q}_{p}$. Suppose $\mathbf{J}=\left\{\mathrm{j}_{0}, \mathrm{j}_{1}, \ldots, \mathrm{j}_{m}\right\}$ is a finite totally ordered set, with

$$
\mathrm{j}_{0}<\mathrm{j}_{1}<\cdots<\mathrm{j}_{m},
$$

such that $g_{\mathrm{j}}=1$ for all $\mathrm{j} \in \mathrm{J}$, and such that

$$
L=L_{\mathrm{j}_{0}} \subsetneq L_{\mathrm{j}_{1}} \subsetneq \cdots \subsetneq L_{\mathrm{j}_{m}}
$$

are proper sublattices of $\frac{1}{p} L$ which are representatives of some self-dual periodic lattice chain considered in [51, Ch. 3] (we can allow general $\mathcal{O} \otimes_{\mathbb{Z}} \mathbb{Q}_{p}$ and consider 
multichains-we focus on the special case here only for the sake of simplicity of exposition). Suppose that $\mathcal{H}=\mathcal{H}^{p} \mathcal{H}_{p}$, where $\mathcal{H}^{p}$ is a neat open compact subgroup of $\mathrm{G}\left(\hat{\mathbb{Z}}^{p}\right)$, and where $\mathcal{H}_{p}:=\bigcap \mathrm{G}_{\mathrm{j}}\left(\mathbb{Z}_{p}\right)$, with $\mathrm{G}_{\mathrm{j}}\left(\mathbb{Z}_{p}\right)$ abusively denoting the image of $\mathrm{G}_{\mathrm{j}}\left(\mathbb{Z}_{p}\right)$ in $\mathrm{G}\left(\mathbb{Q}_{p}\right)$ under the canonical isomorphism $\mathrm{G}_{\mathrm{j}}\left(\mathbb{Q}_{p}\right) \cong$ $\mathrm{G}\left(\mathbb{Q}_{p}\right)$. Then $\mathrm{M}_{\mathcal{H}}$, with its additional structures given by the isomorphisms (2.1), for all $\mathrm{j} \in \mathrm{J}$, parameterizes chains of isogenies

$$
A=A_{\mathrm{j}_{0}} \rightarrow A_{\mathrm{j}_{1}} \rightarrow \cdots \rightarrow A_{\mathrm{j}_{m}} \rightarrow A
$$

(whose composition is the multiplication by $p$ on $A$ ) satisfying certain additional conditions, and extends to a moduli problem over $\overrightarrow{\mathrm{S}}_{0}:=\operatorname{Spec}\left(\mathcal{O}_{F_{0},(p)}\right)$ given by the moduli scheme of chains of isogenies between abelian schemes (with additional PEL structures) as in [51] and later works built on it. In this case, we will study (mixed characteristics) degenerations of such chains of isogenies. While this enriched moduli problem in characteristic zero is canonically isomorphic to $M_{\mathcal{H}}$ and is finite over $M_{\mathcal{H}^{p} \mathrm{G}_{\mathrm{j}}\left(\mathbb{Z}_{p}\right)}$ for each $\mathrm{j} \in \mathrm{J}$, the extended moduli problem in mixed characteristics is in general not finite over any mixed characteristics moduli problem extending $\mathrm{M}_{\mathcal{H}^{p} \mathrm{G}_{\mathrm{j}}\left(\mathbb{Z}_{p}\right)}$.

EXAMPLE 2.5 (Hecke twists). Suppose $\mathbf{J}=\left\{\mathrm{j}_{0}, \mathrm{j}_{1}\right\}$ has two elements, and suppose $\left(g_{\mathrm{j}_{0}}, L_{\mathrm{j}_{0}},\langle\cdot, \cdot\rangle_{\mathrm{j}_{0}}\right)=(1, L,\langle\cdot, \cdot\rangle)$ and $\left(g_{\mathrm{j}_{1}}, L_{\mathrm{j}_{1}},\langle\cdot, \cdot\rangle_{\mathrm{j}_{1}}\right)=(g, L,\langle\cdot, \cdot\rangle)$ for some $g \in \mathrm{G}(\hat{\mathbb{Z}})$. In this case, we will study (mixed characteristics) degenerations of $\mathbb{Q}^{\times}$-isogenies

$$
f:\left(A, \lambda, i, \alpha_{\mathcal{H}}\right) \rightarrow\left(A^{\prime}, \lambda^{\prime}, i^{\prime}, \alpha_{g^{-1} \mathcal{H}_{g}}^{\prime}\right)
$$

realizing the Hecke twists of $\left(A, \lambda, i, \alpha_{\mathcal{H}}\right)$ by $g$ (see [30, Section 6.4.3]). More generally, given any collection $\left\{\left(g_{\mathrm{j}}, L_{\mathrm{j}},\langle\cdot, \cdot\rangle_{\mathrm{j}}\right)\right\}_{\mathrm{j} \in \mathrm{J}^{\prime}}$, we can introduce a twicelarger collection $\left\{\left(g^{\epsilon} g_{\mathrm{j}}, L_{\mathrm{j}},\langle\cdot, \cdot\rangle_{\mathrm{j}}\right)\right\}_{(\epsilon, \mathrm{j}) \in\{0,1\} \times \mathrm{J}^{\prime}}$, provided that both $g_{\mathrm{j}}^{-1} \mathcal{H} g_{\mathrm{j}}$ and $g_{\mathrm{j}}^{-1} g^{-1} \mathcal{H} g g_{\mathrm{j}}$ stabilize the submodule $L_{\mathrm{j}} \otimes_{\mathbb{Z}} \hat{\mathbb{Z}}$ of $L \otimes_{\mathbb{Z}} \mathbb{A}^{\infty}$, for each $\mathrm{j} \in \mathrm{J}^{\prime}$. In this case, we will study (mixed characteristics) degenerations of $\mathbb{Q}^{\times}$-isogenies

$$
f_{\mathrm{j}}:\left(A_{\mathrm{j}}, \lambda_{\mathrm{j}}, i_{\mathrm{j}}, \alpha_{\mathcal{H}_{\mathrm{j}}}\right) \rightarrow\left(A_{\mathrm{j}}^{\prime}, \lambda_{\mathrm{j}}^{\prime}, i_{\mathrm{j}}^{\prime}, \alpha_{g^{-1} \mathcal{H}_{\mathrm{j}} g}^{\prime}\right)
$$

realizing the Hecke twists of $\left(A_{\mathrm{j}}, \lambda_{\mathrm{j}}, i_{\mathrm{j}}, \alpha_{\mathcal{H}_{\mathrm{j}}}\right)$, for all $\mathrm{j} \in \mathrm{J}^{\prime}$, which are related to each other via $\mathbb{Q}^{\times}$-isogenies.

In what follows, we shall fix the choice of a rational prime number $p>0$, and we shall assume that the image $\mathcal{H}^{p}$ of $\mathcal{H}$ under the canonical homomorphism $\mathrm{G}(\hat{\mathbb{Z}}) \rightarrow \mathrm{G}\left(\hat{\mathbb{Z}}^{p}\right)$ is neat (which means, a fortiori, that $\mathcal{H}$ is also neat). Then $\mathcal{H}_{\mathrm{j}}$ and its image $\mathcal{H}_{\mathrm{j}}^{p}$ under the canonical morphism $\mathrm{G}_{\mathrm{j}}(\hat{\mathbb{Z}}) \rightarrow \mathrm{G}_{\mathrm{j}}\left(\hat{\mathbb{Z}}^{p}\right)$ are also neat, for every $\mathrm{j} \in \mathrm{J}$. 
We suggest that first-time readers take, for simplicity, $\mathbf{J}=\left\{\mathrm{j}_{0}\right\}$ (a singleton) and $\left(g_{\mathrm{j}_{0}}, L_{\mathrm{j}_{0}},\langle\cdot, \cdot\rangle_{\mathrm{j}_{0}}\right)=(1, L,\langle\cdot, \cdot\rangle)$ (with no nontrivial Hecke twist) as in Example 2.3, and ignore all the indices $\mathrm{j}$ (and products indexed by them) in the exposition. Furthermore, they might assume that the pairing $\langle\cdot, \cdot\rangle$ is self-dual at $p$, so that Zarhin's trick is not needed. The key points are already novel under these two simplifying assumptions.

\section{Quasiisogeny twists of degenerations}

In this section, let us fix the choice of an index $\mathrm{j} \in \mathrm{J}$.

Let $V$ be a complete discrete valuation ring with fraction field $K$ and algebraically closed residue field $k$. Suppose that there exists a morphism

$$
\eta:=\operatorname{Spec}(K) \rightarrow \mathrm{M}_{\mathcal{H}}
$$

By abuse of notation, let us denote by $\left(G_{\eta}, \lambda_{\eta}, i_{\eta}, \alpha_{\mathcal{H}, \eta}\right)$ the pullback of the tautological object over $\mathrm{M}_{\mathcal{H}}$ under this morphism. By the semistable reduction theorem (see, for example, [30, Theorem 3.3.2.4]), up to replacing $K$ with a finite extension field and replacing $V$ accordingly, we may assume that $G_{\eta}$ extends to a semiabelian scheme $G$ over $\operatorname{Spec}(V)$. By the theory of Néron models (see [4]; cf. [52, IX, 1.4], [10, Ch. I, Proposition 2.7], or [30, Proposition 3.3.1.5]), $\left(G_{\eta}, \lambda_{\eta}, i_{\eta}, \alpha_{\mathcal{H}, \eta}\right)$ extends to a degenerating family $\left(G, \lambda, i, \alpha_{\mathcal{H}}\right)$ of type $\mathrm{M}_{\mathcal{H}}$ over $\operatorname{Spec}(V)$, where $\alpha_{\mathcal{H}}$ is defined only over $\eta=\operatorname{Spec}(K)$, which defines an object of $\mathrm{DEG}_{\mathrm{PEL}, \mathrm{M}_{\mathcal{H}}}(V)$ corresponding to a tuple

$$
\left(B, \lambda_{B}, i_{B}, \underline{X}, \underline{Y}, \phi, c, c^{\vee}, \tau,\left[\alpha_{\mathcal{H}}^{\natural}\right]\right)
$$

in $\mathrm{DD}_{\mathrm{PEL}, \mathrm{M}_{\mathcal{H}}}(V)$ under [30, Theorem 5.3.1.19]. Since the base ring $V$ is strict local, the étale sheaves $\underline{X}$ and $\underline{Y}$ are necessarily constant, which we shall denote by $X$ and $Y$, respectively.

Let $\bar{\eta} \rightarrow \mathrm{M}_{\mathcal{H}}$ be any geometric point above $\eta \rightarrow \mathrm{M}_{\mathcal{H}}$. Then $\alpha_{\mathcal{H}, \eta}$ can be identified with the $\pi_{1}(\eta, \bar{\eta})$-invariant $\mathcal{H}$-orbit of some symplectic isomorphism

$$
\hat{\alpha}_{\bar{\eta}}: L \underset{\mathbb{Z}}{\otimes} \hat{\mathbb{Z}} \stackrel{\sim}{\rightarrow} \mathrm{T} G_{\bar{\eta}}
$$

(cf. [30, Lemma 1.3.6.5]), which induces the $\pi_{1}(\eta, \bar{\eta})$-invariant $\mathcal{H}$-orbit of the induced symplectic isomorphism

$$
\hat{\alpha}_{\bar{\eta}} \underset{\hat{\mathbb{Z}}}{\otimes} \mathbb{A}^{\infty}: L \underset{\mathbb{Z}}{\otimes} \mathbb{A}^{\infty} \stackrel{\sim}{\rightarrow} \mathrm{V} G_{\bar{\eta}} .
$$

The image of $L_{\mathrm{j}} \otimes_{\mathbb{Z}} \hat{\mathbb{Z}}$ under $\left(\hat{\alpha}_{\bar{\eta}} \otimes_{\hat{\mathbb{Z}}} \mathbb{A}^{\infty}\right) \circ g_{\mathrm{j}}: L \otimes_{\mathbb{Z}} \mathbb{A}^{\infty} \stackrel{\sim}{\rightarrow} \mathrm{V} G_{\bar{\eta}}$ is an open compact subgroup of $\mathrm{V} G_{\bar{\eta}}$, which is $\pi_{1}(\eta, \bar{\eta})$-invariant because the preimage $\mathcal{H}_{\mathrm{j}}$ 
of $g_{\mathrm{j}}^{-1} \mathcal{H} g_{\mathrm{j}}$ under the canonical isomorphism $\mathrm{G}_{\mathrm{j}}\left(\mathbb{A}^{\infty}\right) \cong \mathrm{G}\left(\mathbb{A}^{\infty}\right)$ is contained in $\mathrm{G}_{\mathrm{j}}(\hat{\mathbb{Z}})$. Hence, by [30, Lemma 1.3.5.2], we have a $\mathbb{Q}^{\times}$-isogeny

$$
f_{\mathrm{j}, \eta}:\left(G_{\eta}, \lambda_{\eta}, i_{\eta}\right) \rightarrow\left(G_{\mathrm{j}, \eta}, \lambda_{\mathrm{j}, \eta}, i_{\mathrm{j}, \eta}\right)
$$

over $\eta$, together with the $\pi_{1}(\eta, \bar{\eta})$-invariant $\mathcal{H}_{\mathrm{j}}$-orbit of a symplectic isomorphism

$$
\hat{\alpha}_{\mathrm{j}, \bar{\eta}}: L_{\mathrm{j}} \underset{\mathbb{Z}}{\otimes} \hat{\mathbb{Z}} \stackrel{\sim}{\rightarrow} \mathrm{T} G_{\mathrm{j}, \bar{\eta}}
$$

such that the induced symplectic isomorphism

$$
\hat{\alpha}_{\mathrm{j}, \bar{\eta}} \underset{\hat{\mathbb{Z}}}{\otimes} \mathbb{A}^{\infty}: L_{\mathrm{j}} \underset{\mathbb{Z}}{\otimes} \mathbb{A}^{\infty} \stackrel{\sim}{\rightarrow} \mathrm{V} G_{\mathrm{j}, \bar{\eta}}
$$

satisfies the characterizing property

$$
\hat{\alpha}_{\mathrm{j}, \bar{\eta}} \underset{\hat{\mathbb{Z}}}{\otimes} \mathbb{A}^{\infty}=\mathrm{V}\left(f_{\mathrm{j}}\right) \circ\left(\hat{\alpha}_{\bar{\eta}} \underset{\hat{\mathbb{Z}}}{\otimes} \mathbb{A}^{\infty}\right) \circ g_{\mathrm{j}} .
$$

Up to replacing $\lambda_{\mathrm{j}, \bar{\eta}}$ with a $\mathbb{Q}_{>0}^{\times}$-multiple, the $\pi_{1}(\eta, \bar{\eta})$-invariant $\mathcal{H}_{\mathrm{j}}$-orbit of $\hat{\alpha}_{\mathrm{j}, \bar{\eta}}$ induces a level- $\mathcal{H}_{\mathrm{j}}$ structure $\alpha_{\mathcal{H}_{\mathrm{j}}, \eta}$ for $\left(G_{\mathrm{j}, \eta}, \lambda_{\mathrm{j}, \eta}, i_{\mathrm{j}, \eta}\right)$, which defines an object $\left(G_{\mathrm{j}, \eta}, \lambda_{\mathrm{j}, \eta}, i_{\mathrm{j}, \eta}, \alpha_{\mathcal{H}_{\mathrm{j}, \eta}}\right)$ of $\mathrm{M}_{\mathcal{H}_{\mathrm{j}}}(\eta)$ parameterized by a morphism $\eta \rightarrow \mathrm{M}_{\mathcal{H}_{\mathrm{j}}}$. By the proofs of [30, Proposition 1.4.3.4 and Corollary 1.4.3.8], this is just the composition of $\eta \rightarrow \mathrm{M}_{\mathcal{H}}$ with (2.1).

By the theory of Néron models again, the above $\mathbb{Q}^{\times}$-isogeny $f_{\mathrm{j}, \eta}$ extends to a $\mathbb{Q}^{\times}$-isogeny

$$
f_{\mathrm{j}}:(G, \lambda, i) \rightarrow\left(G_{\mathrm{j}}, \lambda_{\mathrm{j}}, i_{\mathrm{j}}\right) .
$$

Together with the level- $\mathcal{H}_{\mathrm{j}}$ structure $\alpha_{\mathcal{H}_{\mathrm{j}, \eta}}$ defined only over $\eta$, which we abusively denote by $\alpha_{\mathcal{H}_{\mathrm{j}}}$, we obtain a degenerating family $\left(G_{\mathrm{j}}, \lambda_{\mathrm{j}}, i_{\mathrm{j}}, \alpha_{\mathcal{H}_{\mathrm{j}}}\right)$ of type $\mathrm{M}_{\mathcal{H}_{\mathrm{j}}}$ over $\operatorname{Spec}(V)$, which defines an object of $\operatorname{DEG}_{\mathrm{PEL}, \mathrm{M}_{\mathcal{H}_{\mathrm{j}}}}(V)$ corresponding to a tuple

$$
\left(B_{\mathrm{j}}, \lambda_{B_{\mathrm{j}}}, i_{B_{\mathrm{j}}}, \underline{X}_{\mathrm{j}}, \underline{Y}_{\mathrm{j}}, \phi_{\mathrm{j}}, c_{\mathrm{j}}, c_{\mathrm{j}}^{\vee}, \tau_{\mathrm{j}},\left[\alpha_{\mathcal{H}_{\mathrm{j}}}^{\natural}\right]\right)
$$

in $\mathrm{DD}_{\mathrm{PEL}, \mathrm{M}_{\mathcal{H}_{\mathrm{j}}}}(V)$ under [30, Theorem 5.3.1.19]. Again, the étale sheaves $\underline{X}_{\mathrm{j}}$ and $\underline{Y}_{\mathrm{j}}$ are necessarily constant, which we shall denote by $X_{\mathrm{j}}$ and $Y_{\mathrm{j}}$, respectively.

Using the canonical isomorphism (2.1), and using the equivalences of categories given by [30, Theorem 5.3.1.19] as above, we know that the object $\left(B, \lambda_{B}, i_{B}, X, Y, \phi, c, c^{\vee}, \tau,\left[\alpha_{\mathcal{H}}^{\natural}\right]\right)$ in $\mathrm{DD}_{\mathrm{PEL}, \mathrm{M}_{\mathcal{H}}}(V)$ determines and is determined by the object $\left(B_{\mathrm{j}}, \lambda_{B_{\mathrm{j}}}, i_{B_{\mathrm{j}}}, X_{\mathrm{j}}, Y_{\mathrm{j}}, \phi_{\mathrm{j}}, c_{\mathrm{j}}, c_{\mathrm{j}}^{\vee}, \tau_{\mathrm{j}},\left[\alpha_{\mathcal{H}_{\mathrm{j}}}^{\natural}\right]\right)$ in $\mathrm{DD}_{\mathrm{PEL}, \mathrm{M}_{\mathcal{H}_{\mathrm{j}}}}(V)$. For our purpose, we will need a more explicit relation between these objects. Let

$$
\alpha_{\mathcal{H}}^{\natural}=\left(\mathrm{Z}_{\mathcal{H}}, \varphi_{-2, \mathcal{H}}^{\sim}, \varphi_{-1, \mathcal{H}}, \varphi_{0, \mathcal{H}}^{\sim}, \delta_{\mathcal{H}}, c_{\mathcal{H}}, c_{\mathcal{H}}^{\vee}, \tau_{\mathcal{H}}\right)
$$

be any representative of $\left[\alpha_{\mathcal{H}}^{\natural}\right]$. 
By construction, $Z_{\mathcal{H}}$ is the $\mathcal{H}$-orbit of the pullback $\mathrm{Z}$ of the geometric filtration $0 \subset \mathrm{T} T_{\bar{\eta}} \subset \mathrm{T} G_{\bar{\eta}}^{\natural} \subset \mathrm{T} G_{\bar{\eta}}$ under $\hat{\alpha}_{\bar{\eta}}: L \otimes_{\mathbb{Z}} \hat{\mathbb{Z}} \stackrel{\sim}{\rightarrow} \mathrm{T} G_{\bar{\eta}}$. Since $\hat{\alpha}_{\mathrm{j}, \bar{\eta}} \otimes_{\hat{\mathbb{Z}}} \mathbb{A}^{\infty}=$ $\mathrm{V}\left(f_{\mathrm{j}, \bar{\eta}}\right) \circ\left(\hat{\alpha}_{\bar{\eta}} \otimes_{\hat{\mathbb{Z}}} \mathbb{A}^{\infty}\right) \circ g_{\mathrm{j}}$, the pullback $\mathrm{Z}_{\mathrm{j}}$ of the geometric filtration $0 \subset \mathrm{T} T_{\bar{\eta}} \subset$ $\mathrm{T} G_{\bar{\eta}}^{\natural} \subset \mathrm{T} G_{\bar{\eta}}$ under $\hat{\alpha}_{\bar{\eta}}: L \otimes_{\mathbb{Z}} \hat{\mathbb{Z}} \stackrel{\sim}{\rightarrow} \mathrm{T} G_{\bar{\eta}}$ is related to $\mathrm{z}$ by

$$
\mathrm{Z}_{\mathrm{j},-i}=\left(g_{\mathrm{j}}^{-1}\left(\mathrm{Z}_{-i} \underset{\hat{\mathbb{Z}}}{\otimes} \mathbb{A}^{\infty}\right)\right) \cap\left(L_{\mathrm{j}} \underset{\mathbb{Z}}{\otimes} \hat{\mathbb{Z}}\right)
$$

for all $i$, whose $\mathcal{H}_{\mathrm{j}}$-orbit is independent of the choices, which we abusively denote by $Z_{\mathcal{H}_{\mathrm{j}}}$ (this notation is abusive because $Z_{\mathcal{H}_{\mathrm{j}}}$ is the $\mathcal{H}_{\mathrm{j}}$-orbit of $Z_{\mathrm{j}}$, not $\mathrm{Z}$ ). Thus, we have a well-defined assignment

$$
\mathrm{Z}_{\mathcal{H}} \mapsto \mathrm{Z}_{\mathcal{H}_{\mathrm{j}}}
$$

which is bijective because $Z_{\mathcal{H}}$ is also determined by $Z_{\mathcal{H}_{\mathrm{j}}}$. By construction, we have isomorphisms

$$
g_{\mathrm{j}}: \mathrm{Z}_{-i} \underset{\hat{\mathbb{Z}}}{\otimes} \mathbb{A}^{\infty} \stackrel{\sim}{\rightarrow} \mathrm{Z}_{\mathrm{j},-i} \underset{\hat{\mathbb{Z}}}{\otimes} \mathbb{A}^{\infty}
$$

for all $i$, which induce isomorphisms

$$
\mathrm{Gr}_{-i}\left(g_{\mathrm{j}}\right): \mathrm{Gr}_{-i}^{\mathrm{z}} \underset{\hat{\mathbb{Z}}}{\otimes} \mathbb{A}^{\infty} \stackrel{\sim}{\rightarrow} \mathrm{Gr}_{\mathrm{j},-i}^{\mathrm{Z}} \underset{\hat{\mathbb{Z}}}{\otimes} \mathbb{A}^{\infty} .
$$

With such an isomorphism for $i=-1$, by the analogs of [30, Proposition 1.4.3.4 and Corollary 1.4.3.8] for the abelian parts of the objects, we obtain a canonical isomorphism

$$
\mathrm{M}_{\mathcal{H}}^{Z_{\mathcal{H}}} \stackrel{\sim}{\rightarrow} \mathrm{M}_{\mathcal{H}_{\mathrm{j}}}^{Z_{\mathcal{H}_{\mathrm{j}}}}
$$

(over $\mathrm{S}_{0}=\operatorname{Spec}\left(F_{0}\right)$ ) such that the tautological object $\left(B, \lambda_{B}, i_{B}, \varphi_{-1, \mathcal{H}}\right)$ over $\mathrm{M}_{\mathcal{H}}^{Z \mathcal{H}}$ and the pullback of the tautological object $\left(B_{\mathrm{j}}, \lambda_{B_{\mathrm{j}}}, i_{B_{\mathrm{j}}}, \varphi_{-1, \mathcal{H}_{\mathrm{j}}}\right)$ over $\mathrm{M}_{\mathcal{H}_{\mathrm{j}}}^{\mathrm{Z \mathcal {H } _ { \mathrm { j } }}}$ are related via a $\mathbb{Q}^{\times}$-isogeny $f_{\mathrm{j}, B}:\left(B, \lambda_{B}, i_{B}\right) \rightarrow\left(B_{\mathrm{j}}, \lambda_{B_{\mathrm{j}}}, i_{B_{\mathrm{j}}}\right)$ (canonically induced by the above $\mathbb{Q}^{\times}$-isogeny $f_{\mathrm{j}}:(G, \lambda, i) \rightarrow\left(G_{\mathrm{j}}, \lambda_{\mathrm{j}}, i_{\mathrm{j}}\right)$, or rather by the corresponding $\mathbb{Q}^{\times}$-isogeny $f_{\mathrm{j}}^{\natural}:\left(G^{\natural}, \lambda^{\natural}, i^{\natural}\right) \rightarrow\left(G_{\mathrm{j}}^{\natural}, \lambda_{\mathrm{j}}^{\natural}, i_{\mathrm{j}}^{\natural}\right)$ induced by taking Raynaud extensions), where $\varphi_{-1, \mathcal{H}}$ (respectively $\varphi_{-1, \mathcal{H}_{\mathrm{j}}}$ ) is induced by the $\pi_{1}(\eta$, $\bar{\eta})$-invariant $\mathcal{H}$-orbit of $\varphi_{-1}:=\mathrm{Gr}_{-1}\left(\hat{\alpha}_{\bar{\eta}}\right): \mathrm{Gr}_{-1}^{\mathrm{z}} \stackrel{\sim}{\rightarrow} \mathrm{T} B_{\bar{\eta}} \cong\left(\mathrm{T} G_{\bar{\eta}}^{\natural}\right) /\left(\mathrm{T} T_{\bar{\eta}}\right)$ (respectively $\mathcal{H}_{\mathrm{j}}$-orbit of $\varphi_{\mathrm{j},-1}:=\mathrm{Gr}_{-1}\left(\hat{\alpha}_{\mathrm{j}, \bar{\eta}}\right): \mathrm{Gr}_{-1}^{Z_{\mathrm{j}}} \stackrel{\sim}{\rightarrow} \mathrm{T} B_{\mathrm{j}, \bar{\eta}} \cong\left(\mathrm{T} G_{\mathrm{j}, \bar{\eta}}^{\natural}\right) /\left(\mathrm{T} T_{\mathrm{j}, \bar{\eta}}\right)$ ).

By construction, $X$ and $Y$ are the character groups of the torus parts $T$ and $T^{\vee}$ of $G^{\natural}$ and $G^{\vee, \natural}$, respectively. Consider the submodule $\left(v\left(g_{\mathrm{j}}\right)^{-1} \mathrm{Gr}_{-2}\left(g_{\mathrm{j}}\right)\right)\left(\mathrm{Gr}_{-2}^{\mathrm{Z}_{\mathrm{j}}}\right)$ of $\mathrm{Gr}_{-2}^{Z} \otimes_{\hat{\mathbb{Z}}} \mathrm{A}^{\infty}$. By [30, Lemmas 5.4.3.6 and 5.4.3.7], there exists a unique $\mathcal{O}$-lattice $X_{\mathrm{j}}$ in $X \otimes_{\mathbb{Z}} \mathbb{Q}$, together with the canonical isomorphism $f_{\mathrm{j}, X}: X_{\mathrm{j}} \otimes_{\mathbb{Z}} \mathbb{Q} \stackrel{\sim}{\rightarrow} X \otimes_{\mathbb{Z}} \mathbb{Q}$ 
and a canonically induced isomorphism $\varphi_{\mathrm{j},-2}: \mathrm{Gr}_{-2}^{Z_{\mathrm{j}}} \stackrel{\sim}{\rightarrow} \operatorname{Hom}_{\hat{\mathbb{Z}}}\left(X_{\mathrm{j}} \otimes_{\mathbb{Z}} \hat{\mathbb{Z}}, \hat{\mathbb{Z}}(1)\right)$ such that

$$
\varphi_{\mathrm{j},-2} \underset{\hat{\mathbb{Z}}}{\otimes} \mathbb{A}^{\infty}=\left({ }^{t} f_{\mathrm{j}, X} \underset{\mathbb{Z}}{\otimes} \mathbb{A}^{\infty}\right) \circ\left(\varphi_{-2} \underset{\mathbb{\mathbb { Z }}}{\otimes} \mathbb{A}^{\infty}\right) \circ\left(v\left(g_{\mathrm{j}}\right)^{-1} \mathrm{Gr}_{-2}\left(g_{\mathrm{j}}\right)\right) .
$$

Similarly, there exists a unique $\mathcal{O}$-lattice $Y_{\mathrm{j}}$ in $Y \otimes_{\mathbb{Z}} \mathbb{Q}$, together with the canonical isomorphism $f_{\mathrm{j}, Y}: Y \otimes_{\mathbb{Z}} \mathbb{Q} \stackrel{\sim}{\rightarrow} Y_{\mathrm{j}} \otimes_{\mathbb{Z}} \mathbb{Q}$ and a canonically induced isomorphism $\varphi_{\mathrm{j}, 0}: \mathrm{Gr}_{0}^{Z_{\mathrm{j}}} \stackrel{\sim}{\rightarrow} Y_{\mathrm{j}} \otimes_{\mathbb{Z}} \hat{\mathbb{Z}}$ such that

$$
\varphi_{\mathrm{j}, 0} \underset{\hat{\mathbb{Z}}}{\otimes} \mathbb{A}^{\infty}=\left(f_{\mathrm{j}, Y} \underset{\mathbb{Z}}{\otimes} \mathbb{A}^{\infty}\right) \circ\left(\varphi_{0} \underset{\hat{\mathbb{Z}}}{\otimes} \mathbb{A}^{\infty}\right) \circ\left(\mathrm{Gr}_{0}\left(g_{\mathrm{j}}\right)\right) .
$$

Then $X_{\mathrm{j}}$ and $Y_{\mathrm{j}}$ are canonically isomorphic to the character groups of the torus parts $T_{\mathrm{j}}$ and $T_{\mathrm{j}}^{\vee}$ of $G_{\mathrm{j}}^{\natural}$ and $G_{\mathrm{j}}^{\vee, \natural}$, respectively, such that the morphisms $f_{\mathrm{j}, X}$ and $f_{\mathrm{j}, Y}$ are induced by the $\mathbb{Q}^{\times}$-isogenies $f_{\mathrm{j}, T}: T \rightarrow T_{\mathrm{j}}$ and $f_{\mathrm{j}, T^{\vee}}: T_{\mathrm{j}}^{\vee} \rightarrow T^{\vee}$ induced by $f_{\mathrm{j}}: G \rightarrow G_{\mathrm{j}}$ and $f_{\mathrm{j}}^{\vee}: G_{\mathrm{j}}^{\vee} \rightarrow G^{\vee}$, respectively. By abuse of notation, let $\left(\varphi_{-2, \mathcal{H}_{\mathrm{j}}}, \varphi_{0, \mathcal{H}_{\mathrm{j}}}\right)$ be induced by the $\mathcal{H}_{\mathrm{j}}$-orbit of $\left(\varphi_{\mathrm{j},-2}, \varphi_{\mathrm{j}, 0}\right)$, and let $\left(\varphi_{-2, \mathcal{H}_{\mathrm{j}}}^{\sim}, \varphi_{-1, \mathcal{H}_{\mathrm{j}}}\right.$, $\left.\tilde{\varphi_{0, \mathcal{H}_{\mathrm{j}}}}\right)$ be induced by the $\mathcal{H}_{\mathrm{j}}$-orbit of $\left(\varphi_{\mathrm{j},-2}, \varphi_{\mathrm{j},-1}, \varphi_{\mathrm{j}, 0}\right)$.

Consider the unique $r_{\mathrm{j}} \in \mathbb{Q}_{>0}^{\times}$such that $v(g)\left(\langle\cdot, \cdot\rangle \otimes_{\mathbb{Z}} \mathbb{A}^{\infty}\right)=r_{\mathrm{j}} u\left(\langle\cdot, \cdot\rangle_{\mathrm{j}} \otimes_{\mathbb{Z}} \mathbb{A}^{\infty}\right)$ for some $u \in \hat{\mathbb{Z}}^{\times}$. Then there is a unique homomorphism $\phi_{\mathrm{j}}: Y_{\mathrm{j}} \hookrightarrow X_{\mathrm{j}}$ such that $\phi_{\mathrm{j}} \otimes_{\mathbb{Z}} \mathbb{Q}=r_{\mathrm{j}}^{-1} f_{\mathrm{j}, X}^{-1} \circ\left(\phi \otimes_{\mathbb{Z}} \mathbb{Q}\right) \circ f_{\mathrm{j}, Y}^{-1}$, which is induced by the pairing $\mathrm{Gr}_{-2}^{\mathrm{Z}_{\mathrm{j}}} \times \mathrm{Gr}_{0}^{\mathrm{Z}_{\mathrm{j}}} \rightarrow$ $\hat{\mathbb{Z}}(1)$ induced by $\langle\cdot, \cdot\rangle_{\mathrm{j}}: L_{\mathrm{j}} \times L_{\mathrm{j}} \rightarrow \mathbb{Z}(1)$.

Thus we have obtained well-defined assignments of torus arguments

$$
\Phi_{\mathcal{H}}=\left(X, Y, \phi, \varphi_{-2, \mathcal{H}}, \varphi_{0, \mathcal{H}}\right) \mapsto \Phi_{\mathcal{H}_{\mathrm{j}}}=\left(X_{\mathrm{j}}, Y_{\mathrm{j}}, \phi_{\mathrm{j}}, \varphi_{-2, \mathcal{H}_{\mathrm{j}}}, \varphi_{0, \mathcal{H}_{\mathrm{j}}}\right)
$$

and of the orbits above the abelian parts

$$
\Phi_{\mathcal{H}}^{\sim}=\left(X, Y, \phi, \varphi_{-2, \mathcal{H}}^{\sim}, \tilde{\varphi_{0, \mathcal{H}}}\right) \mapsto \Phi_{\mathcal{H}_{\mathrm{j}}}^{\sim}=\left(X_{\mathrm{j}}, Y_{\mathrm{j}}, \phi_{\mathrm{j}}, \varphi_{-2, \mathcal{H}_{\mathrm{j}}}^{\sim}, \varphi_{0, \mathcal{H}_{\mathrm{j}}}^{\sim}\right),
$$

where the latter induces the former. By the above construction and by the definition of $\Gamma_{\Phi_{\mathcal{H}}}$ and $\Gamma_{\Phi_{\mathcal{H}_{\mathrm{j}}}}$ as the respective automorphism groups of $\Phi_{\mathcal{H}}$ and $\Phi_{\mathcal{H}_{\mathrm{j}}}$ (see [30, Definitions 6.2.4.1 and 5.4.1.6]), we also obtain a canonical isomorphism

$$
\Gamma_{\Phi_{\mathcal{H}}} \stackrel{\sim}{\rightarrow} \Gamma_{\Phi_{\mathcal{H}_{\mathrm{j}}}}
$$

Accordingly, we have a canonical isomorphism

$$
\mathrm{M}_{\mathcal{H}}^{\Phi_{\mathcal{H}}} \stackrel{\sim}{\rightarrow} \mathrm{M}_{\mathcal{H}_{\mathrm{j}}}^{\Phi_{\mathcal{H}_{\mathrm{j}}}}
$$

(over $\mathrm{S}_{0}=\operatorname{Spec}\left(F_{0}\right)$ ) compatible with (3.2) and equivariant with (3.5), which matches the object $\left(\varphi_{-2, \mathcal{H}}^{\sim}, \varphi_{0, \mathcal{H}}^{\sim}\right)$ parameterized by $\mathrm{M}_{\mathcal{H}}^{\Phi_{\mathcal{H}}} \rightarrow \mathrm{M}_{\mathcal{H}}^{Z \mathcal{H}}$ with the object $\left(\varphi_{-2, \mathcal{H}_{\mathrm{j}}}^{\sim}, \varphi_{0, \mathcal{H}_{\mathrm{j}}}^{\sim}\right)$ parameterized by $\mathrm{M}_{\mathcal{H}_{\mathrm{j}}}^{\Phi_{\mathcal{H}_{\mathrm{j}}}} \rightarrow \mathrm{M}_{\mathcal{H}_{\mathrm{j}}}^{\mathrm{ZH}_{\mathcal{H}_{\mathrm{j}}}}$. 
If we take any splitting $\delta_{\mathrm{j}}$ of $Z_{\mathrm{j}}$, and abusively denote its $\mathcal{H}_{\mathrm{j}}$-orbit by $\delta_{\mathcal{H}_{\mathrm{j}}}$, then we obtain a cusp label $\left[\left(\mathrm{Z}_{\mathcal{H}_{\mathrm{j}}}, \Phi_{\mathcal{H}_{\mathrm{j}}}, \delta_{\mathcal{H}_{\mathrm{j}}}\right)\right]$. Thus we have a well-defined assignment

$$
\left[\left(\mathrm{Z}_{\mathcal{H}}, \Phi_{\mathcal{H}}, \delta_{\mathcal{H}}\right)\right] \mapsto\left[\left(\mathrm{Z}_{\mathcal{H}_{\mathrm{j}}}, \Phi_{\mathcal{H}_{\mathrm{j}}}, \delta_{\mathcal{H}_{\mathrm{j}}}\right)\right]
$$

Let us fix once and for all the choices of $\delta_{\mathrm{j}}$ for all pairs $\left(\mathrm{Z}_{\mathrm{j}}, \Phi_{\mathrm{j}}\right)$, which determine the choices of $\delta_{\mathcal{H}_{\mathrm{j}}}$ for all pairs $\left(Z_{\mathcal{H}_{\mathrm{j}}}, \Phi_{\mathcal{H}_{\mathrm{j}}}\right)$. Then the assignment (3.7) is induced by an assignment of representatives of cusp labels

$$
\left(\mathrm{Z}_{\mathcal{H}}, \Phi_{\mathcal{H}}, \delta_{\mathcal{H}}\right) \mapsto\left(\mathrm{Z}_{\mathcal{H}_{\mathrm{j}}}, \Phi_{\mathcal{H}_{\mathrm{j}}}, \delta_{\mathcal{H}_{\mathrm{j}}}\right) .
$$

By the definition of the group $\mathbf{S}_{\Phi_{\mathcal{H}}}$ (see [30, (6.2.3.5), Convention 6.2.3.20, Lemma 6.2.4.4]) and by [28, Corollary 3.6.10], we can identify elements of $\operatorname{Hom}\left(\mathbf{S}_{\Phi_{\mathcal{H}}}, \hat{\mathbb{Z}}(1)\right)$ with maps in $Y \otimes_{\mathbb{Z}} \hat{\mathbb{Z}} \rightarrow \operatorname{Hom}_{\hat{\mathbb{Z}}}\left(X \otimes_{\mathbb{Z}} \hat{\mathbb{Z}}, \hat{\mathbb{Z}}(1)\right)$ (satisfying certain additional conditions), and we can identify elements $H \in \operatorname{Hom}\left(\mathbf{S}_{\Phi_{\mathcal{H}}}, \mathbb{Z}(1)\right)$ with elements $h \in \mathcal{H}$ which induce maps

$$
\mathrm{Gr}_{-2}^{\mathrm{Z}} \oplus \mathrm{Gr}_{-1}^{\mathrm{z}} \oplus \mathrm{Gr}_{0}^{\mathrm{z}}=\mathrm{Gr}^{\mathrm{z}} \stackrel{\stackrel{\delta}{\rightarrow}}{\rightarrow} L \underset{\mathbb{Z}}{\otimes} \hat{\mathbb{Z}} \stackrel{h}{\rightarrow} L \underset{\mathbb{Z}}{\otimes} \underset{\mathbb{Z}}{\stackrel{\delta^{-1}}{\rightarrow}} \mathrm{Gr}^{\mathrm{z}}=\mathrm{Gr}_{-2}^{\mathrm{z}} \oplus \mathrm{Gr}_{-1}^{\mathrm{z}} \oplus \mathrm{Gr}_{0}^{\mathrm{Z}}
$$

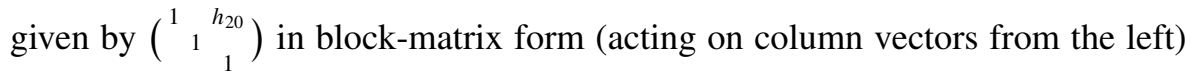
with $h_{20}: \mathrm{Gr}_{0}^{\mathrm{Z}} \rightarrow \mathrm{Gr}_{-2}^{\mathrm{Z}}$ induced by

$$
\mathrm{Gr}_{0}^{\mathrm{Z}} \stackrel{\varphi_{0}}{\rightarrow} Y \underset{\mathbb{Z}}{\otimes} \hat{\mathbb{Z}} \stackrel{h \otimes_{\mathbb{Z}} \hat{\mathbb{Z}}}{\rightarrow} \operatorname{Hom}_{\hat{\mathbb{Z}}}(X \underset{\mathbb{Z}}{\otimes} \hat{\mathbb{Z}}, \hat{\mathbb{Z}}(1)) \stackrel{\varphi_{-2}^{-1}}{\stackrel{\sim}{\rightarrow}} \mathrm{Gr}_{-2}^{\mathrm{z}} .
$$

This identification depends not on the choice of $\delta$, but on the choices of $Z$ and $\left(\varphi_{-2}, \varphi_{0}\right)$, which is canonical (only) up to the action of $\Gamma_{\Phi_{\mathcal{H}}}$. Similarly, we have an identification between elements $H_{\mathrm{j}} \in \operatorname{Hom}\left(\mathbf{S}_{\Phi_{\mathcal{H}_{\mathrm{j}}}}, \mathbb{Z}(1)\right)$ and elements $h_{\mathrm{j}} \in \mathcal{H}_{\mathrm{j}}$, depending not on the choice of $\delta_{\mathrm{j}}$, but on the choices of $z_{\mathrm{j}}$ and $\left(\varphi_{\mathrm{j},-2}, \varphi_{\mathrm{j}, 0}\right)$, which is canonical (only) up to the action of $\Gamma_{\Phi_{\mathcal{H}_{\mathrm{j}}}}$. Nevertheless, if $z_{\mathrm{j}}$ and $\left(\varphi_{\mathrm{j},-2}, \varphi_{\mathrm{j}, 0}\right)$ are determined by $\mathrm{Z}$ and $\left(\varphi_{-2}, \varphi_{0}\right)$ as above, then the above identifications are compatible with the isomorphisms $\mathcal{H} \stackrel{\sim}{\rightarrow} g_{\mathrm{j}}^{-1} \mathcal{H} g_{\mathrm{j}} \stackrel{\sim}{\rightarrow} \mathcal{H}_{\mathrm{j}}$ (by definition of $\mathcal{H}_{\mathrm{j}}$ ), and we have a canonical isomorphism $\operatorname{Hom}\left(\mathbf{S}_{\Phi_{\mathcal{H}}}, \mathbb{Z}(1)\right) \stackrel{\sim}{\rightarrow} \operatorname{Hom}\left(\mathbf{S}_{\Phi_{\mathcal{H}}}, \mathbb{Z}(1)\right)$ of abelian groups equivariant with (3.5), which induces canonical isomorphisms

$$
\begin{aligned}
& \mathbf{S}_{\Phi_{\mathcal{H}}} \stackrel{\sim}{\rightarrow} \mathbf{S}_{\Phi_{\mathcal{H}}}, \\
& \left(\mathbf{S}_{\Phi_{\mathcal{H}_{\mathrm{j}}}}\right)_{\mathbb{Q}}:=\mathbf{S}_{\Phi_{\mathcal{H}_{\mathrm{j}}}} \underset{\mathbb{Z}}{\otimes} \mathbb{Q} \stackrel{\sim}{\rightarrow}\left(\mathbf{S}_{\Phi_{\mathcal{H}}}\right)_{\mathbb{Q}}:=\mathbf{S}_{\Phi_{\mathcal{H}}} \underset{\mathbb{Z}}{\otimes} \mathbb{Q}, \\
& \left(\mathbf{S}_{\Phi_{\mathcal{H}}}\right)_{\mathbb{Q}}^{\vee} \stackrel{\sim}{\rightarrow}\left(\mathbf{S}_{\Phi_{\mathcal{H}_{\mathrm{j}}}}\right)_{\mathbb{Q}}^{\vee}, \\
& \left(\mathbf{S}_{\Phi_{\mathcal{H}}}\right)_{\mathbb{R}}^{\vee} \stackrel{\sim}{\rightarrow}\left(\mathbf{S}_{\Phi_{\mathcal{H}_{\mathrm{j}}}}\right)_{\mathbb{R}}^{\vee},
\end{aligned}
$$


and

$$
E_{\Phi_{\mathcal{H}}}=\operatorname{Hom}\left(\mathbf{S}_{\Phi_{\mathcal{H}}}, \mathbf{G}_{\mathrm{m}}\right) \stackrel{\sim}{\rightarrow} E_{\Phi_{\mathcal{H}_{\mathrm{j}}}}=\operatorname{Hom}\left(\mathbf{S}_{\Phi_{\mathcal{H}_{\mathrm{j}}}}, \mathbf{G}_{\mathrm{m}}\right),
$$

which are compatible with each other and compatibly equivariant with (3.5). If we identify elements of $\left(\mathbf{S}_{\Phi_{\mathcal{H}}}\right)_{\mathbb{Q}}^{\vee}$ (respectively $\left.\left(\mathbf{S}_{\Phi_{\mathcal{H}_{\mathrm{j}}}}\right)_{\mathbb{Q}}^{\vee}\right)$ with Hermitian pairings on $Y \otimes_{\mathbb{Z}} \mathbb{Q}$ (respectively $Y_{\mathrm{j}} \otimes_{\mathbb{Z}} \mathbb{Q}$ ) as in [30, Section 6.2.5], then (3.11) is defined by pullback under the inverse of $f_{\mathrm{j}, Y}: Y \otimes_{\mathbb{Z}} \mathbb{Q} \stackrel{\sim}{\rightarrow} Y_{\mathrm{j}} \otimes_{\mathbb{Z}} \mathbb{Q}$. Hence (3.12) preserves the positive definiteness and semidefiniteness of pairings, and maps $\mathbf{P}_{\Phi_{\mathcal{H}}}$ (respectively $\mathbf{P}_{\Phi_{\mathcal{H}}}^{+}$) to $\mathbf{P}_{\Phi_{\mathcal{H}_{\mathrm{j}}}}$ (respectively $\mathbf{P}_{\Phi_{\mathcal{H}_{\mathrm{j}}}}^{+}$).

By [30, Lemma 5.4.2.11], the representative $\left(Z_{\mathcal{H}_{\mathrm{j}}}, \Phi_{\mathcal{H}_{\mathrm{j}}}, \delta_{\mathcal{H}_{\mathrm{j}}}\right)$ of the cusp label $\left[\left(\mathrm{Z}_{\mathcal{H}_{\mathrm{j}}}, \Phi_{\mathcal{H}_{\mathrm{j}}}, \delta_{\mathcal{H}_{\mathrm{j}}}\right)\right]$ uniquely determines a representative

$$
\alpha_{\mathcal{H}_{\mathrm{j}}}^{\natural}=\left(\mathrm{Z}_{\mathcal{H}_{\mathrm{j}}}, \varphi_{-2, \mathcal{H}_{\mathrm{j}}}^{\sim}, \varphi_{-1, \mathcal{H}_{\mathrm{j}}}, \stackrel{\sim}{\sim} \mathcal{H}_{\mathrm{H}}, \delta_{\mathcal{H}_{\mathrm{j}}}, c_{\mathcal{H}_{\mathrm{j}}}, c_{\mathcal{H}_{\mathrm{j}}}^{\vee}, \tau_{\mathcal{H}_{\mathrm{j}}}\right)
$$

of $\left[\alpha_{\mathcal{H}_{\mathrm{j}}}^{\natural}\right]$, where $\mathrm{Z}_{\mathcal{H}_{\mathrm{j}}}, \varphi_{-2, \mathcal{H}_{\mathrm{j}}}^{\sim}, \varphi_{-1, \mathcal{H}_{\mathrm{j}}}, \varphi_{0, \mathcal{H}_{\mathrm{j}}}^{\sim}$, and $\delta_{\mathcal{H}_{\mathrm{j}}}$ are as above. It remains to relate $\left(c_{\mathcal{H}_{\mathrm{j}}}, c_{\mathcal{H}_{\mathrm{j}}}^{\vee}, \tau_{\mathcal{H}_{\mathrm{j}}}\right)$ to $\left(c_{\mathcal{H}}, c_{\mathcal{H}}^{\vee}, \tau_{\mathcal{H}}\right)$. For this purpose, let us also fix some representatives $\mathrm{Z}, \Phi=\left(X, Y, \phi, \varphi_{-2}, \varphi_{0}\right)$, and $\varphi_{-1}$ in their $\mathcal{H}$-orbits, which induce $\mathrm{Z}_{\mathrm{j}}, \Phi_{\mathrm{j}}=\left(X_{\mathrm{j}}, Y_{\mathrm{j}}, \phi_{\mathrm{j}}, \varphi_{\mathrm{j},-2}, \varphi_{\mathrm{j}, 0}\right)$, and $\varphi_{\mathrm{j},-1}$ by the procedures explained above.

By comparing the universal properties (as in [31, Lemma 1.3.2.11 and Proposition 1.3.2.12]) of $C_{\Phi_{\mathcal{H}}, \delta_{\mathcal{H}}} \rightarrow \mathrm{M}_{\mathcal{H}}^{\Phi_{\mathcal{H}}}$ and $C_{\Phi_{\mathcal{H}_{\mathrm{j}}}, \delta_{\mathcal{H}_{\mathrm{j}}}} \rightarrow \mathrm{M}_{\mathcal{H}_{\mathrm{j}}}^{\Phi_{\mathcal{H}_{\mathrm{j}}}}$, there is a canonical isomorphism

$$
C_{\Phi_{\mathcal{H}}, \delta_{\mathcal{H}}} \stackrel{\sim}{\rightarrow} C_{\Phi_{\mathcal{H}_{\mathrm{j}}}, \delta_{\mathcal{H}_{\mathrm{j}}}}
$$

(over $\mathrm{S}_{0}=\operatorname{Spec}\left(F_{0}\right)$ ) compatible with (3.6) and equivariant with (3.5), which matches the object $\left(c_{\mathcal{H}}, c_{\mathcal{H}}^{\vee}\right)$ parameterized by $C_{\Phi_{\mathcal{H}}, \delta_{\mathcal{H}}} \rightarrow \mathrm{M}_{\mathcal{H}}^{\Phi_{\mathcal{H}}}$ with the object $\left(c_{\mathcal{H}_{\mathrm{j}}}, c_{\mathcal{H}_{\mathrm{j}}}^{\vee}\right)$ parameterized by $C_{\Phi_{\mathcal{H}_{\mathrm{j}}}, \delta_{\mathcal{H}_{\mathrm{j}}}} \rightarrow \mathrm{M}_{\mathcal{H}_{\mathrm{j}}}^{\Phi_{\mathcal{H}_{\mathrm{j}}}}$. Concretely, by [31, Lemma 1.3.2.11 and Proposition 1.3.2.12], the pair $\left(c_{\mathcal{H}}, c_{\mathcal{H}}^{\vee}\right)$ is equivalent to a tuple

$$
\left(G_{\eta}^{\natural}, \lambda_{\eta}^{\natural}: G_{\eta}^{\natural} \rightarrow G_{\eta}^{\vee, \natural}, i_{\eta}^{\natural}, \beta_{\mathcal{H}, \eta}^{\natural}\right)
$$

over $\eta$, where the subtuple $\left(G_{\eta}^{\natural}, \lambda_{\eta}^{\natural}: G_{\eta}^{\natural} \rightarrow G_{\eta}^{\vee, \natural}, i_{\eta}^{\natural}\right)$ is determined by two homomorphisms $c_{\eta}: Y \rightarrow B_{\eta}^{\vee}$ and $c_{\eta}^{\vee}: X \rightarrow B_{\eta}$ compatible with each other under the homomorphisms $\phi: Y \hookrightarrow X$ and $\lambda_{B_{\eta}}: B_{\eta} \rightarrow B_{\eta}^{\vee}$, and where $\beta_{\mathcal{H}, \eta}^{\natural}$ is equivalent to the $\pi_{1}(\eta, \bar{\eta})$-invariant $\mathcal{H}$-orbit of a triple

$$
\hat{\beta}_{\bar{\eta}}^{\natural}=\left(\hat{\beta}_{\bar{\eta}}^{\natural, 0}: \mathrm{Z}_{-1} \stackrel{\sim}{\rightarrow} \mathrm{T} G_{\bar{\eta}}^{\natural}, \hat{\beta}_{\bar{\eta}}^{\natural, \#, 0}: \mathrm{Z}_{-1}^{\#} \stackrel{\sim}{\rightarrow} \mathrm{T} G_{\bar{\eta}}^{\vee, \natural}, \hat{v}_{\bar{\eta}}^{\natural}: \hat{\mathbb{Z}}(1) \stackrel{\sim}{\rightarrow} \mathrm{T} \mathbf{G}_{\mathrm{m}, \bar{\eta}}\right),
$$

where $\mathrm{Z}^{\#}$ is the filtration on the dual lattice $L^{\#} \otimes_{\mathbb{Z}} \hat{\mathbb{Z}}$ defined by

$$
\mathrm{Z}_{-i}^{\#}:=\left(\underset{\hat{\mathbb{Z}}}{\mathrm{Z}_{-i} \underset{\mathbb{A}}{\otimes}} \mathbb{A}^{\infty}\right) \cap\left(L_{\mathbb{Z}}^{\#} \underset{\mathbb{Z}}{\otimes}\right)
$$


for each $i$, such that $\hat{\beta}_{\bar{\eta}}^{\natural, 0}$ and $\hat{\beta}_{\bar{\eta}}^{\natural, \#, 0}$ are compatible with each other under the canonical morphisms induced by $\mathrm{Z}_{-i} \rightarrow \mathrm{Z}_{-i}^{\#}$ and $\lambda^{\natural}$, inducing the above-chosen $\varphi_{-2}, \varphi_{-1}$, and $\varphi_{0}$ on the graded pieces, and such that $\hat{\nu}_{\bar{\eta}}^{\natural}=v\left(\varphi_{-1}\right)$. Under the homomorphism $\left(\hat{\beta}_{\bar{\eta}}^{\natural, 0} \otimes_{\hat{\mathbb{Z}}} \mathbb{A}^{\infty}\right) \circ g_{\mathrm{j}}: g_{\mathrm{j}}^{-1}\left(\mathrm{Z}_{-1} \otimes_{\hat{\mathbb{Z}}} \mathbb{A}^{\infty}\right) \stackrel{\sim}{\rightarrow} \mathrm{V} G_{\bar{\eta}}^{\natural}$, the image of $\mathrm{Z}_{\mathrm{j},-1}=\left(g_{\mathrm{j}}^{-1}\left(\mathrm{Z}_{-1} \otimes_{\hat{\mathbb{Z}}} \mathbb{A}^{\infty}\right)\right) \cap\left(L_{\mathrm{j}} \otimes_{\mathbb{Z}} \hat{\mathbb{Z}}\right)$ is a $\pi_{1}(\eta, \bar{\eta})$-invariant open compact subgroup of $\mathrm{V} G_{\bar{\eta}}^{\natural}$, which induces a $\mathbb{Q}^{\times}$-isogeny $f_{\mathrm{j}, \eta}^{\natural}: G_{\eta}^{\natural} \rightarrow G_{\mathrm{j}, \eta}^{\natural}$. Similarly, under the homomorphism $\left(\hat{\beta}_{\bar{\eta}}^{\natural, \#, 0} \otimes_{\hat{\mathbb{Z}}} \mathbb{A}^{\infty}\right) \circ\left(v\left(g_{\mathrm{j}}\right)^{-1} g_{\mathrm{j}}\right): g_{\mathrm{j}}^{-1}\left(\mathrm{Z}_{-1} \otimes_{\hat{\mathbb{Z}}} \mathbb{A}^{\infty}\right) \stackrel{\sim}{\rightarrow} \mathrm{V} G_{\bar{\eta}}^{\vee, \natural}$, the image of $Z_{\mathrm{j},-1}^{\#}=\left(g_{\mathrm{j}}^{-1}\left(\mathrm{Z}_{-1} \otimes_{\hat{\mathbb{Z}}} \mathbb{A}^{\infty}\right)\right) \cap\left(L_{\mathrm{j}}^{\#} \otimes_{\mathbb{Z}} \hat{\mathbb{Z}}\right)$ is a $\pi_{1}(\eta, \bar{\eta})$-invariant open compact subgroup of $\mathrm{V} G_{\bar{\eta}}^{\vee, \natural}$, which induces a $\mathbb{Q}^{\times}$-isogeny $\left(f_{\mathrm{j}, \eta}^{\vee, \natural}\right)^{-1}: G_{\eta}^{\vee, \natural} \rightarrow$ $G_{\mathrm{j}, \eta}^{\vee, \natural}$. Here $G_{\mathrm{j}, \eta}^{\natural}$ and $G_{\mathrm{j}, \eta}^{\vee, \natural}$ are determined by two homomorphisms $c_{\mathrm{j}, \eta}: Y_{\mathrm{j}} \rightarrow B_{\mathrm{j}, \eta}^{\vee}$ and $c_{\mathrm{j}, \eta}^{\vee}: X \rightarrow B_{\mathrm{j}, \eta}$ compatible with each other under the homomorphisms $\phi_{\mathrm{j}}: Y_{\mathrm{j}} \hookrightarrow X_{\mathrm{j}}$ and $\lambda_{B_{\mathrm{j}, \eta}}: B_{\mathrm{j}, \eta} \rightarrow B_{\mathrm{j}, \eta}^{\vee}$, which induce a homomorphism

$$
\lambda_{\mathrm{j}, \eta}^{\natural}: G_{\mathrm{j}, \eta}^{\natural} \rightarrow G_{\mathrm{j}, \eta}^{\vee, \natural} .
$$

Let

$$
\hat{\beta}_{\mathrm{j}, \bar{\eta}}^{\natural, 0}: \mathrm{Z}_{\mathrm{j},-1} \stackrel{\sim}{\rightarrow} \mathrm{T} G_{\mathrm{j}, \bar{\eta}}^{\natural}
$$

and

$$
\hat{\beta}_{\mathrm{j}, \bar{\eta}}^{\natural, \#, 0}: \mathrm{Z}_{\mathrm{j},-1}^{\#} \stackrel{\sim}{\rightarrow} \mathrm{T} G_{\bar{\eta}}^{\vee, \natural}
$$

denote the induced isomorphisms, and let

$$
\hat{v}_{\mathrm{j}, \bar{\eta}}^{\natural}:=v\left(\varphi_{\mathrm{j},-1}\right) .
$$

Then the $\mathcal{H}_{\mathrm{j}}$-orbit of

$$
\hat{\beta}_{\mathrm{j}, \bar{\eta}}^{\natural}:=\left(\hat{\beta}_{\mathrm{j}, \bar{\eta}}^{\natural, 0}: \mathrm{Z}_{\mathrm{j},-1} \stackrel{\sim}{\rightarrow} \mathrm{T} G_{\mathrm{j}, \bar{\eta}}^{\natural}, \hat{\beta}_{\mathrm{j}, \bar{\eta}}^{\natural, \#, 0}: \mathrm{z}_{\mathrm{j},-1}^{\#} \stackrel{\sim}{\rightarrow} \mathrm{T} G_{\bar{\eta}}^{\vee, \natural}, \hat{v}_{\mathrm{j}, \bar{\eta}}^{\natural}: \hat{\mathbb{Z}}(1) \stackrel{\sim}{\rightarrow} \mathrm{T} \mathbf{G}_{\mathrm{m}, \bar{\eta}}\right)
$$

is $\pi_{1}(\eta, \bar{\eta})$-invariant and induces the $\beta_{\mathcal{H}_{\mathrm{j}}, \eta}^{\natural}$ such that the tuple

$$
\left(G_{\mathrm{j}, \eta}^{\natural}, \lambda_{\mathrm{j}, \eta}^{\natural}: G_{\mathrm{j}, \eta}^{\natural} \rightarrow G_{\mathrm{j}, \eta}^{\vee, \natural}, i_{\mathrm{j}, \eta}^{\natural}, \beta_{\mathcal{H}_{\mathrm{j}}, \eta}^{\natural}\right)
$$

over $\eta$ corresponds to the pair $\left(c_{\mathcal{H}_{\mathrm{j}}}, c_{\mathcal{H}_{\mathrm{j}}}^{\vee}\right.$ ) (note that the choices of $\delta$ and $\delta_{\mathrm{j}}$ play no role in the comparison between $\hat{\beta}_{\bar{\eta}}^{\natural}$ and $\left.\hat{\beta}_{\mathrm{j}, \bar{\eta}}^{\natural}\right)$.

Similarly, by comparing the universal properties (as in [31, Lemma 1.3.2.28 and Proposition 1.3.2.31]) of $\Xi_{\Phi_{\mathcal{H}}, \delta_{\mathcal{H}}} \rightarrow C_{\Phi_{\mathcal{H}}, \delta_{\mathcal{H}}}$ and $\Xi_{\Phi_{\mathcal{H}_{\mathrm{j}}}, \delta_{\mathcal{H}_{\mathrm{j}}}} \rightarrow C_{\Phi_{\mathcal{H}_{\mathrm{j}}}, \delta_{\mathcal{H}_{\mathrm{j}}}}$, there is a canonical isomorphism

$$
\Xi_{\Phi_{\mathcal{H}}, \delta_{\mathcal{H}}} \stackrel{\sim}{\rightarrow} \Xi_{\Phi_{\mathcal{H}_{\mathrm{j}}}, \delta_{\mathcal{H}_{\mathrm{j}}}}
$$


(over $\mathrm{S}_{0}=\operatorname{Spec}\left(F_{0}\right)$ ) compatible with (3.14) and equivariant with (3.5) and (3.13), which matches the object $\tau_{\mathcal{H}}$ parameterized by $\Xi_{\Phi_{\mathcal{H}}, \delta_{\mathcal{H}}} \rightarrow C_{\Phi_{\mathcal{H}}, \delta_{\mathcal{H}}}$ with the object $\tau_{\mathcal{H}_{\mathrm{j}}}$ parameterized by $\Xi_{\Phi_{\mathcal{H}_{\mathrm{j}}}, \delta_{\mathcal{H}_{\mathrm{j}}}} \rightarrow C_{\Phi_{\mathcal{H}_{\mathrm{j}}}, \delta_{\mathcal{H}_{\mathrm{j}}}}$. Concretely, by [31, Lemma 1.3.2.28 and Proposition 1.3.2.31], the triple $\left(c_{\mathcal{H}}, c_{\mathcal{H}}^{\vee}, \tau_{\mathcal{H}}\right)$ is equivalent to a tuple

$$
\left(G_{\eta}^{\natural}, \lambda_{\eta}^{\natural}: G_{\eta}^{\natural} \rightarrow G_{\eta}^{\vee, \natural}, i_{\eta}^{\natural}, \tau_{\eta}, \beta_{\mathcal{H}, \eta}\right)
$$

over $\eta$. Here the subtuple $\left(G_{\eta}^{\natural}, \lambda_{\eta}^{\natural}, i_{\eta}^{\natural}\right)$ is as in the previous paragraph, and $\tau_{\eta}$ : $\mathbf{1}_{Y \times X, \eta} \stackrel{\sim}{\rightarrow}\left(c_{\eta}^{\vee} \times_{\eta} c_{\eta}\right)^{*} \mathcal{P}_{B_{\eta}}^{\otimes(-1)}$ is a trivialization of biextensions which induces two homomorphisms $\iota_{\eta}: Y \rightarrow G_{\eta}^{\natural}$ and $\iota_{\eta}^{\vee}: X \rightarrow G_{\eta}^{\vee, \natural}$ compatible with each other under the homomorphisms $\phi: Y \hookrightarrow X$ and $\lambda_{\eta}^{\natural}: G_{\eta}^{\natural} \rightarrow G_{\eta}^{\vee, \natural}$, which allow us to recover the modules $\mathrm{T} G_{\bar{\eta}}$ and $\mathrm{T} G_{\bar{\eta}}^{\vee}$ as extensions of $Y \otimes_{\mathbb{Z}} \hat{\mathbb{Z}}$ and $X \otimes_{\mathbb{Z}} \hat{\mathbb{Z}}$ by $\mathrm{T} G_{\bar{\eta}}^{\natural}$ and $\mathrm{T} G_{\bar{\eta}}^{\vee, \natural}$, respectively, together with the morphism $\mathrm{T}\left(\lambda_{\bar{\eta}}\right): \mathrm{T} G_{\bar{\eta}} \rightarrow \mathrm{T} G_{\bar{\eta}}^{\vee}$ inducing $\phi \otimes_{\mathbb{Z}} \hat{\mathbb{Z}}$ and $\mathrm{T}\left(\lambda_{\bar{\eta}}^{\natural}\right)$ on the graded pieces, without having to recover $G_{\bar{\eta}}$, $G_{\bar{\eta}}^{\vee}$, and $\lambda_{\bar{\eta}}: G_{\bar{\eta}} \rightarrow G_{\bar{\eta}}^{\vee}$ themselves. Based on these, $\beta_{\mathcal{H}, \eta}$ is equivalent to the $\pi_{1}(\eta, \bar{\eta})$-invariant $\mathcal{H}$-orbit of a triple

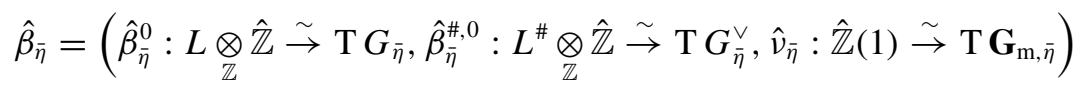

such that $\hat{\beta}_{\bar{\eta}}^{0}$ and $\hat{\beta}_{\bar{\eta}}^{\#, 0}$ are compatible with each other under the canonical morphisms induced by $L \rightarrow L^{\#}$ and $\mathrm{T}\left(\lambda_{\bar{\eta}}\right)$, which induce the above-chosen $\varphi_{-2}, \varphi_{-1}$, and $\varphi_{0}$ on the graded pieces, and such that $\hat{v}_{\bar{\eta}}=v\left(\varphi_{-1}\right)$. Such a triple $\hat{\beta}_{\bar{\eta}}$ induces a triple $\hat{\beta}_{\bar{\eta}}^{\natural}$ as above; in particular, it induces the $\mathbb{Q}^{\times}$-isogenies $f_{\mathrm{j}, \eta}^{\natural}: G_{\eta}^{\natural} \rightarrow G_{\mathrm{j}, \eta}^{\natural}$ and $\left(f_{\mathrm{j}, \eta}^{\vee, \natural}\right)^{-1}: G_{\eta}^{\vee, \natural} \rightarrow G_{\mathrm{j}, \eta}^{\vee, \natural}$ in the previous paragraph. Together with the isomorphisms $f_{\mathrm{j}, X}: X_{\mathrm{j}} \otimes_{\mathbb{Z}} \mathbb{Q} \stackrel{\sim}{\rightarrow} X \otimes_{\mathbb{Z}} \mathbb{Q}$ and $f_{\mathrm{j}, Y}: Y \otimes_{\mathbb{Z}} \mathbb{Q} \stackrel{\sim}{\rightarrow} Y_{\mathrm{j}} \otimes_{\mathbb{Z}} \mathbb{Q}$, they induce a trivialization $\tau_{\mathrm{j}, \eta}: \mathbf{1}_{Y_{\mathrm{j}} \times X_{\mathrm{j}, \eta}} \stackrel{\sim}{\rightarrow}\left(c_{\mathrm{j}, \eta}^{\vee} \times{ }_{\eta} c_{\mathrm{j}, \eta}\right)^{*} \mathcal{P}_{B_{\mathrm{j}, \eta}}^{\otimes(-1)}$ of biextensions which induces two homomorphisms $\iota_{\mathrm{j}, \eta}: Y \rightarrow G_{\eta}^{\natural}$ and $\iota_{\mathrm{j}, \eta}^{\vee}: X \rightarrow G_{\eta}^{\vee, \natural}$ compatible with each other under the homomorphisms $\phi_{\mathrm{j}}: Y_{\mathrm{j}} \hookrightarrow X_{\mathrm{j}}$ and $\lambda_{\mathrm{j}, \eta}^{\natural}: G_{\mathrm{j}, \eta}^{\natural} \rightarrow G_{\mathrm{j}, \eta}^{\vee, \natural}$, which allows us to recover the modules $\mathrm{T} G_{\mathrm{j}, \bar{\eta}}$ and $\mathrm{T} G_{\mathrm{j}, \bar{\eta}}^{\vee}$ as extensions of $Y_{\mathrm{j}} \otimes_{\mathbb{Z}} \hat{\mathbb{Z}}$ and $X_{\mathrm{j}} \otimes_{\mathbb{Z}} \hat{\mathbb{Z}}$ by $\mathrm{T} G_{\mathrm{j}, \bar{\eta}}^{\natural}$ and $\mathrm{T} G_{\mathrm{j}, \bar{\eta}}^{\vee, \natural}$, respectively, together with the morphism $\mathrm{T}\left(\lambda_{\mathrm{j}, \bar{\eta}}\right): \mathrm{T} G_{\mathrm{j}, \bar{\eta}} \rightarrow \mathrm{T} G_{\mathrm{j}, \bar{\eta}}^{\vee}$ inducing $\phi_{\mathrm{j}} \otimes_{\mathbb{Z}} \hat{\mathbb{Z}}$ and $\mathrm{T}\left(\lambda_{\mathrm{j}, \bar{\eta}}^{\natural}\right)$ on the graded pieces, without having to recover $G_{\mathrm{j}, \bar{\eta}}, G_{\mathrm{j}, \bar{\eta}}^{\vee}$, and $\lambda_{\mathrm{j}, \bar{\eta}}: G_{\mathrm{j}, \bar{\eta}} \rightarrow G_{\mathrm{j}, \bar{\eta}}^{\vee}$ themselves; and to recover the canonical isomorphisms $\mathrm{V}\left(f_{\mathrm{j}, \bar{\eta}}\right): \mathrm{V} G_{\bar{\eta}} \rightarrow \mathrm{V} G_{\mathrm{j}, \bar{\eta}}$ and $\mathrm{V}\left(f_{\mathrm{j}, \bar{\eta}}^{\vee}\right)^{-1}$ : $\mathrm{V} G_{\bar{\eta}}^{\vee} \rightarrow \mathrm{V} G_{\mathrm{j}, \bar{\eta}}^{\vee}$, without having to recover the $\mathbb{Q}^{\times}$-isogenies $f_{\mathrm{j}, \bar{\eta}}: G_{\bar{\eta}} \rightarrow G_{\mathrm{j}, \bar{\eta}}$ and $\left(f_{\mathrm{j}, \bar{\eta}}^{\vee}\right)^{-1}: G_{\bar{\eta}}^{\vee} \rightarrow G_{\mathrm{j}, \bar{\eta}}^{\vee}$ themselves. Let

$$
\hat{\beta}_{\mathrm{j}, \bar{\eta}}^{0}: L_{\mathrm{j}} \underset{\mathbb{Z}}{\otimes} \hat{\mathbb{Z}} \stackrel{\sim}{\rightarrow} \mathrm{T} G_{\mathrm{j}, \bar{\eta}}
$$


denote the restriction of

$$
\mathrm{V}\left(f_{\mathrm{j}, \bar{\eta}}\right) \circ\left(\hat{\beta}_{\bar{\eta}}^{0} \underset{\hat{\mathbb{Z}}}{\otimes} \mathbb{A}^{\infty}\right) \circ g_{\mathrm{j}}: L \underset{\hat{\mathbb{Z}}}{\otimes} \mathbb{A}^{\infty} \stackrel{\sim}{\rightarrow} \mathrm{V} G_{\mathrm{j}, \bar{\eta}}^{\natural}
$$

to $L_{\mathrm{j}} \otimes_{\mathbb{Z}} \hat{\mathbb{Z}}$, and let

$$
\hat{\beta}_{\mathrm{j}, \bar{\eta}}^{\#, 0}: L_{\mathrm{j}}^{\#} \underset{\mathbb{Z}}{\otimes} \hat{\mathbb{Z}} \stackrel{\sim}{\rightarrow} \mathrm{T} G_{\bar{\eta}}^{\vee}
$$

denote the restriction of

$$
\mathrm{V}\left(f_{\mathrm{j}, \bar{\eta}}^{\vee}\right)^{-1} \circ\left(\hat{\beta}_{\bar{\eta}}^{\#, 0} \underset{\hat{\mathbb{Z}}}{\otimes} \mathbb{A}^{\infty}\right) \circ\left(\nu\left(g_{\mathrm{j}}\right)^{-1} g_{\mathrm{j}}\right): L^{\#} \underset{\mathbb{Z}}{\otimes} \mathbb{A}^{\infty} \stackrel{\sim}{\rightarrow} \mathrm{V} G_{\mathrm{j}, \bar{\eta}}^{\vee}
$$

to $L_{\mathrm{j}}^{\#} \otimes_{\mathbb{Z}} \hat{\mathbb{Z}}$ (the images of these restrictions are $\mathrm{T} G_{\mathrm{j}, \bar{\eta}}$ and $\mathrm{T} G_{\mathrm{j}, \bar{\eta}}^{\vee}$, respectively, by checking the images on the graded pieces). Let

$$
\hat{v}_{\mathrm{j}, \bar{\eta}}:=v\left(\varphi_{\mathrm{j},-1}\right) .
$$

Then the $\mathcal{H}_{\mathrm{j}}$-orbit of

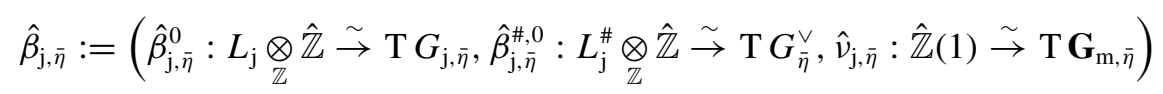

is $\pi_{1}(\eta, \bar{\eta})$-invariant and induces the $\beta_{\mathcal{H}_{\mathrm{j}}, \eta}$ such that the tuple

$$
\left(G_{\mathrm{j}, \eta}^{\natural}, \lambda_{\mathrm{j}, \eta}^{\natural}: G_{\mathrm{j}, \eta}^{\natural} \rightarrow G_{\mathrm{j}, \eta}^{\vee, \natural}, i_{\mathrm{j}, \eta}^{\natural}, \tau_{\mathrm{j}, \eta}, \beta_{\mathcal{H}_{\mathrm{j}, \eta}}\right)
$$

over $\eta$ corresponds to the triple $\left(c_{\mathcal{H}_{\mathrm{j}}}, c_{\mathcal{H}_{\mathrm{j}}}^{\vee}, \tau_{\mathcal{H}_{\mathrm{j}}}\right)$.

LEMMA 3.16. If $v: \operatorname{Inv}(V) \rightarrow \mathbb{Z}$ is the homomorphism induced by the discrete valuation of $V$, where $\operatorname{Inv}(V)$ denotes the group of invertible $V$-submodules of $K$, and if we denote by $B: \mathbf{S}_{\Phi_{\mathcal{H}}} \rightarrow \operatorname{Inv}(V)$ (respectively $B_{\mathrm{j}}: \mathbf{S}_{\Phi_{\mathcal{H}_{\mathrm{j}}}} \rightarrow \operatorname{Inv}(V)$ ) the homomorphism defined by $\tau_{\mathcal{H}}$ (respectively $\tau_{\mathrm{j}}$ ), or rather $\tau_{\eta}$ (respectively $\tau_{\mathrm{j}, \eta}$ ), as in [30, Construction 6.3.1.1], then (3.11) maps the element $v \circ B: \mathbf{S}_{\Phi_{\mathcal{H}}} \rightarrow \mathbb{Z}$ of $\mathbf{S}_{\Phi_{\mathcal{H}}}^{\vee}$ to $a \mathbb{Q}_{>0}^{\times}$-multiple of the element $v \circ B_{\mathrm{j}}: \mathbf{S}_{\Phi_{\mathcal{H}_{\mathrm{j}}}} \rightarrow \mathbb{Z}$ of $\mathbf{S}_{\Phi_{\mathcal{H}_{\mathrm{j}}}}^{\vee}$

Proof. This follows from the above argument. Alternatively, it suffices to note that the collection of all multiples of $\iota_{\eta}: Y_{\eta} \rightarrow G_{\eta}^{\natural}$ determines the collection of all multiples of $\iota_{\mathrm{j}, \eta}: Y_{\mathrm{j}, \eta} \rightarrow G_{\mathrm{j}, \eta}^{\natural}$, via the isomorphism $f_{\mathrm{j}, Y}: Y \otimes_{\mathbb{Z}} \mathbb{Q} \stackrel{\sim}{\rightarrow} Y_{\mathrm{j}} \otimes_{\mathbb{Z}} \mathbb{Q}$ and the $\mathbb{Q}^{\times}$-isogeny $f_{\mathrm{j}, \eta}^{\natural}: G_{\eta}^{\natural} \rightarrow G_{\mathrm{j}, \eta}^{\natural}$.

The $E_{\Phi_{\mathcal{H}}}$-torsor structure of $\Xi_{\Phi_{\mathcal{H}}, \delta_{\mathcal{H}}} \rightarrow C_{\Phi_{\mathcal{H}}, \delta_{\mathcal{H}}}$ allows us to identify the pushforward of $\mathscr{O}_{\Xi_{\Phi_{\mathcal{H}}, \delta_{\mathcal{H}}}}$ (under the structural morphism $\Xi_{\Phi_{\mathcal{H}}, \delta_{\mathcal{H}}} \rightarrow C_{\Phi_{\mathcal{H}}, \delta_{\mathcal{H}}}$ ) with 
an $\mathscr{O}_{C_{\Phi_{\mathcal{H}}, \delta_{\mathcal{H}}}}$-algebra given by the direct sum $\bigoplus_{\ell \in \mathbf{S}_{\Phi_{\mathcal{H}}}} \Psi_{\Phi_{\mathcal{H}}, \delta_{\mathcal{H}}}(\ell)$, and allows us to define, for each nondegenerate rational polyhedral cone $\sigma \subset\left(\mathbf{S}_{\Phi_{\mathcal{H}}}\right)_{\mathbb{R}}^{\vee}$, an affine toroidal embedding

$$
\Xi_{\Phi_{\mathcal{H}}, \delta \mathcal{H}} \hookrightarrow \Xi_{\Phi_{\mathcal{H}}, \delta \mathcal{H}}(\sigma):=\underline{\operatorname{Spec}}_{\mathscr{C}_{C_{\Phi_{\mathcal{H}}, \delta \mathcal{H}}}}\left(\bigoplus_{\ell \in \sigma^{\vee}} \Psi_{\Phi_{\mathcal{H}}, \delta \mathcal{H}}(\ell)\right),
$$

where

$$
\sigma^{\vee}:=\left\{\ell \in \mathbf{S}_{\Phi_{\mathcal{H}}}:\langle\ell, y\rangle \geqslant 0 \forall y \in \sigma\right\}
$$

as usual (see [30, Definition 6.1.1.8]). Similarly, for each nondegenerate rational polyhedral cone $\sigma_{\mathrm{j}} \subset\left(\mathbf{S}_{\Phi_{\mathcal{H}_{\mathrm{j}}}}\right)_{\mathbb{R}}^{\vee}$, we have an affine toroidal embedding

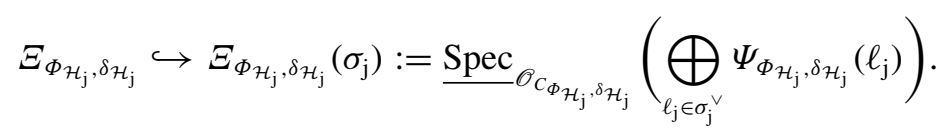

Since (3.15) is equivariant with (3.13), it induces a canonical isomorphism from

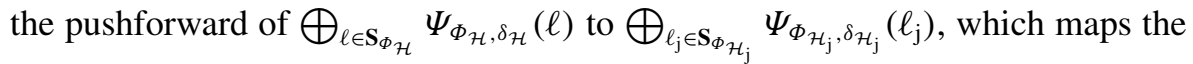
pushforward of $\Psi_{\Phi_{\mathcal{H}}, \delta_{\mathcal{H}}}(\ell)$ to $\Psi_{\Phi_{\mathcal{H}_{\mathrm{j}}}, \delta_{\mathcal{H}_{\mathrm{j}}}}\left(\ell_{\mathrm{j}}\right)$ when $\ell_{\mathrm{j}}$ is mapped to $\ell$ under (3.9). Consequently, if $\sigma_{\mathrm{j}}$ is the image of $\sigma$ under (3.12), in which case $\sigma^{\vee}$ is the image of $\sigma_{\mathrm{j}}^{\vee}$ under (3.9), then the isomorphism (3.15) (necessarily uniquely) extends to an isomorphism

$$
\Xi_{\Phi_{\mathcal{H}}, \delta \mathcal{H}}(\sigma) \stackrel{\sim}{\rightarrow} \Xi_{\Phi_{\mathcal{H}_{\mathrm{j}}}, \delta_{\mathcal{H}_{\mathrm{j}}}}\left(\sigma_{\mathrm{j}}\right)
$$

compatible with (3.14), (3.15), (3.17), and (3.18).

Let

$$
\sigma^{\perp}:=\left\{\ell \in \mathbf{S}_{\Phi_{\mathcal{H}}}:\langle\ell, y\rangle=0 \forall y \in \sigma\right\}
$$

as usual (see [30, Definition 6.1.2.5]) and let $\mathfrak{X}_{\Phi_{\mathcal{H}}, \delta_{\mathcal{H}}, \sigma}$ denote the formal completion of $\Xi_{\Phi_{\mathcal{H}}, \delta_{\mathcal{H}}}(\sigma)$ along the $\sigma$-stratum

$$
\Xi_{\Phi_{\mathcal{H}}, \delta_{\mathcal{H}}, \sigma}:=\underline{\operatorname{Spec}}_{\mathscr{O}_{C_{\Phi_{\mathcal{H}}, \delta_{\mathcal{H}}}}}\left(\bigoplus_{\ell \in \sigma^{\perp}} \Psi_{\Phi_{\mathcal{H}}, \delta_{\mathcal{H}}}(\ell)\right) .
$$

Similarly, let $\mathfrak{X}_{\Phi_{\mathcal{H}_{\mathrm{j}}}, \delta_{\mathcal{H}_{\mathrm{j}}}, \sigma_{\mathrm{j}}}$ denote the formal completion of $\Xi_{\Phi_{\mathcal{H}_{\mathrm{j}}}, \delta_{\mathcal{H}_{\mathrm{j}}}}\left(\sigma_{\mathrm{j}}\right)$ along the $\sigma_{\mathrm{j}}$-stratum

$$
\Xi_{\Phi_{\mathcal{H}_{\mathrm{j}}}, \delta_{\mathcal{H}_{\mathrm{j}}}, \sigma_{\mathrm{j}}}:=\underline{\operatorname{Spec}} \mathscr{O}_{C_{\Phi_{\mathcal{H}_{\mathrm{j}}}, \delta_{\mathcal{H}_{\mathrm{j}}}}}\left(\bigoplus_{\ell_{\mathrm{j}} \in \sigma_{\mathrm{j}}^{\perp}} \Psi_{\Phi_{\mathcal{H}_{\mathrm{j}}}, \delta_{\mathcal{H}_{\mathrm{j}}}}\left(\ell_{\mathrm{j}}\right)\right) .
$$

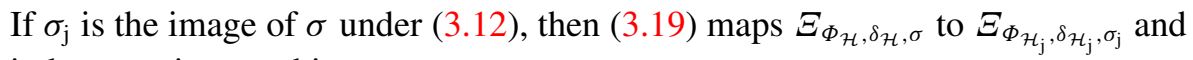
induces an isomorphism

$$
\mathfrak{X}_{\Phi_{\mathcal{H}}, \delta_{\mathcal{H}}, \sigma} \stackrel{\sim}{\rightarrow} \mathfrak{X}_{\Phi_{\mathcal{H}_{\mathrm{j}}}, \delta_{\mathcal{H}_{\mathrm{j}}}, \sigma_{\mathrm{j}}}
$$

compatible with (3.14) and (3.19). 
By the theory of two-step degenerations (see [10, Ch. III, Section 10] and [30, Section 4.5.6]), the above argument also shows that the assignments (3.7) and (3.8) are compatible with the formation of surjections as in [30, Definitions 5.4.2.12 and 5.4.2.13].

LEMMA 3.21. Suppose $\Sigma=\left\{\Sigma_{\Phi_{\mathcal{H}}}\right\}_{\left[\left(\Phi_{\mathcal{H}}, \delta_{\mathcal{H}}\right)\right]}$ is a compatible choice of admissible smooth rational polyhedral cone decomposition data for $\mathrm{M}_{\mathcal{H}}$ as in [30, Condition 6.3.3.2 and Definition 6.3.3.4]. Then there exists a unique compatible choice $\Sigma_{\mathrm{j}}=\left\{\Sigma_{\Phi_{\mathcal{H}_{\mathrm{j}}}}\right\}_{\left[\left(\Phi_{\mathcal{H}_{\mathrm{j}}}, \delta_{\mathcal{H}_{\mathrm{j}}}\right)\right]}$ of admissible smooth rational polyhedral cone decomposition data for $\mathrm{M}_{\mathcal{H}_{\mathrm{j}}}$ such that, for each representative $\left(\mathrm{Z}_{\mathcal{H}}, \Phi_{\mathcal{H}}, \delta_{\mathcal{H}}\right)$ of cusp label for $\mathrm{M}_{\mathcal{H}}$, which induces a representative $\left(\mathrm{Z}_{\mathcal{H}_{\mathrm{j}}}, \Phi_{\mathcal{H}_{\mathrm{j}}}, \delta_{\mathcal{H}_{\mathrm{j}}}\right)$ of cusp label for $\mathrm{M}_{\mathcal{H}_{\mathrm{j}}}$ via (3.8), the cone decomposition $\Sigma_{\Phi_{\mathcal{H}_{\mathrm{j}}}}$ of $\mathbf{S}_{\Phi_{\mathcal{H}_{\mathrm{j}}}}$ is the image of the cone decomposition $\Sigma_{\Phi_{\mathcal{H}}}$ of $\mathbf{S}_{\Phi_{\mathcal{H}}}$ under any isomorphism (3.12) as above. In this case, we say that $\Sigma_{\mathrm{j}}$ is induced by $\Sigma$. Consequently, we also have canonical isomorphisms

$$
\mathrm{M}_{\mathcal{H}, \Sigma}^{\text {tor }} \stackrel{\sim}{\rightarrow} \mathrm{M}_{\mathcal{H}_{\mathrm{j}}, \Sigma_{\mathrm{j}}}^{\text {tor }}
$$

and

$$
\mathrm{M}_{\mathcal{H}}^{\min } \stackrel{\sim}{\rightarrow} \mathrm{M}_{\mathcal{H}_{\mathrm{j}}}^{\min }
$$

(over $\mathrm{S}_{0}=\operatorname{Spec}\left(F_{0}\right)$ ) between the toroidal and minimal compactifications for $\mathrm{M}_{\mathcal{H}}$ and $\mathrm{M}_{\mathcal{H}_{\mathrm{j}}}$ (see [30, Theorems 6.4.1.1 and 7.2.4.1]), which are compatible with each other under the canonical morphisms

$$
\oint_{\mathcal{H}}: \mathrm{M}_{\mathcal{H}, \Sigma}^{\text {tor }} \rightarrow \mathrm{M}_{\mathcal{H}}^{\mathrm{min}}
$$

and

$$
\oint_{\mathcal{H}_{\mathrm{j}}}: \mathrm{M}_{\mathcal{H}_{\mathrm{j}}, \Sigma_{\mathrm{j}}}^{\text {tor }} \rightarrow \mathrm{M}_{\mathcal{H}_{\mathrm{j}}}^{\text {min }},
$$

and with the canonical isomorphism (2.1). When $\left(\mathrm{Z}_{\mathcal{H}}, \Phi_{\mathcal{H}}, \delta_{\mathcal{H}}\right)$ and $\left(\mathrm{Z}_{\mathcal{H}_{\mathrm{j}}}, \Phi_{\mathcal{H}_{\mathrm{j}}}\right.$, $\delta_{\mathcal{H}_{\mathrm{j}}}$ ) are as above such that $\sigma_{\mathrm{j}}$ is the image of $\sigma$ under (3.12), the morphism (3.22) maps the $\left[\left(\mathrm{Z}_{\mathcal{H}}, \delta_{\mathcal{H}}, \sigma\right)\right]$-stratum $\mathrm{Z}_{\left[\left(\mathrm{Z}_{\mathcal{H}}, \delta_{\mathcal{H}}, \sigma\right)\right]}$ of $\mathrm{M}_{\mathcal{H}, \Sigma}^{\text {ter }}$ to the $\left[\left(\mathrm{Z}_{\mathcal{H}_{\mathrm{j}}}, \delta_{\mathcal{H}_{\mathrm{j}}}, \sigma_{\mathrm{j}}\right)\right]$ stratum $\mathrm{Z}_{\left[\left(\mathrm{Z}_{\mathcal{H}_{\mathrm{j}}}, \delta_{\mathcal{H}_{\mathrm{j}}}, \sigma_{\mathrm{j}}\right)\right]}$ of $\mathrm{M}_{\mathcal{H}_{\mathrm{j}}, \Sigma_{\mathrm{j}}}^{\text {tor }}$, and induces a canonical isomorphism

$$
\mathrm{Z}_{\left[\left(\mathrm{Z}_{\mathcal{H}}, \delta_{\mathcal{H}}, \sigma\right)\right]} \stackrel{\sim}{\rightarrow} \mathrm{Z}_{\left[\left(\mathrm{Z}_{\mathcal{H}_{\mathrm{j}}}, \delta_{\mathcal{H}_{\mathrm{j}}}, \sigma_{\mathrm{j}}\right)\right]}
$$

Moreover, under the canonical isomorphisms

$$
\left(\mathrm{M}_{\mathcal{H}, \Sigma}^{\mathrm{tor}}\right)_{\mathrm{Z}_{\left[\left(z_{\mathcal{H}}, \delta_{\mathcal{H}}, \sigma\right)\right]}^{\wedge}}^{\wedge} \cong \mathfrak{X}_{\Phi_{\mathcal{H}}, \delta_{\mathcal{H}}, \sigma}
$$


and

$$
\left(\mathrm{M}_{\mathcal{H}_{\mathrm{j}}, \Sigma_{\mathrm{j}}}^{\text {tor }}\right)_{\mathrm{Z}_{\left[\left(z_{\mathcal{H}_{\mathrm{j}}}, \delta_{\left.\left.\mathcal{H}_{\mathrm{j}}, \sigma_{\mathrm{j}}\right]\right]}\right.\right.}}^{\wedge} \cong \mathfrak{X}_{\Phi_{\mathcal{H}_{\mathrm{j}}}, \delta_{\mathcal{H}_{\mathrm{j}}}, \sigma_{\mathrm{j}}}
$$

given by [30, Theorem 6.4.1.1(5)], the canonical isomorphism

$$
\left(\mathrm{M}_{\mathcal{H}, \Sigma}^{\text {tor }}\right)_{\mathrm{Z}_{\left[\left(Z_{\mathcal{H}}, \delta \mathcal{H}, \sigma\right)\right]}}^{\wedge} \stackrel{\sim}{\rightarrow}\left(\mathrm{M}_{\mathcal{H}_{\mathrm{j}}, \Sigma_{\mathrm{j}}}^{\text {tor }}\right)_{\mathrm{Z}_{\left[\left(Z_{\mathcal{H}_{\mathrm{j}}}, \delta \mathcal{H}_{\mathrm{j}}, \sigma_{\mathrm{j}}\right)\right]}^{\wedge}}^{\wedge}
$$

induced by (3.22) can be identified with the canonical isomorphism (3.20). Accordingly, the morphism (3.23) maps the $\left[\left(\mathrm{Z}_{\mathcal{H}}, \delta_{\mathcal{H}}\right)\right]$-stratum $\mathrm{Z}_{\left[\left(\mathrm{Z}_{\mathcal{H}}, \delta_{\mathcal{H}}\right)\right]}$ of $\mathrm{M}_{\mathcal{H}}^{\min }$ to the $\left[\left(\mathrm{Z}_{\mathcal{H}_{\mathrm{j}}}, \delta_{\mathcal{H}_{\mathrm{j}}}\right)\right]$-stratum $\mathrm{Z}_{\left[\left(\mathrm{Z}_{\mathcal{H}_{\mathrm{j}}}, \delta \mathcal{H}_{\mathrm{j}}\right)\right]}$ of $\mathrm{M}_{\mathcal{H}_{\mathrm{j}}}^{\text {min }}$, and induces a canonical isomorphism

$$
\mathrm{Z}_{\left[\left(\mathrm{Z}_{\mathcal{H}}, \delta \mathcal{H}\right)\right]} \stackrel{\sim}{\rightarrow} \mathrm{Z}_{\left[\left(\mathrm{Z}_{\mathcal{H}_{\mathrm{j}}}, \delta \mathcal{H}_{\mathrm{j}}\right)\right]} .
$$

Proof. The canonical isomorphism (3.22) exists and satisfies the desired properties by comparing the universal properties of $\mathrm{M}_{\mathcal{H}, \Sigma}^{\text {tor }}$ and $\mathrm{M}_{\mathcal{H}_{\mathrm{j}}, \Sigma_{\mathrm{j}}}^{\text {tor }}$ as in [30, Theorem 6.4.1.1(6)], by comparing the induced degeneration data over complete discrete valuation rings, as explained in this section thus far, and by comparing the Mumford families as in the proof of [30, Theorem 6.4.1.1(5)]. Consequently, the canonical isomorphism (3.23) exists and satisfies the desired properties because the minimal compactifications are isomorphic to the respective projective spectra of rings of global sections of powers of Hodge invertible sheaves, as in [30, Theorem 7.2.4.1(3)], and because the stratifications of the minimal compactifications are compatible with those of the toroidal compactifications as in [30, Theorem 7.2.4.1(5)].

\section{Auxiliary choices of smooth moduli problems}

For each $\mathrm{j} \in \mathrm{J}$, let $L_{\mathrm{j}}^{\#}$ denote the dual lattice of $L_{\mathrm{j}}$ in $L_{\mathrm{j}} \otimes_{\mathbb{Z}} \mathbb{Q} \cong L \otimes_{\mathbb{Z}} \mathbb{Q}$ with respect to the pairing $\langle\cdot, \cdot\rangle_{\mathrm{j}}$ valued in $\mathbb{Z}(1)$ (as in [30, Definition 1.1.4.11]).

Lemma 4.1. Suppose $\mathrm{j} \in \mathrm{J}$. For each integer $d_{\mathrm{j}} \geqslant 1$, there exist integers $a_{\mathrm{j}, 1}>0$ and $a_{\mathrm{j}, 2} \geqslant 0$, and a positive definite symmetric bilinear pairing

$$
(\cdot, \cdot)_{\mathrm{j}}: \mathbb{Z}^{\oplus\left(a_{\mathrm{j}, 1}+a_{\mathrm{j}, 2}\right)} \times \mathbb{Z}^{\oplus\left(a_{\mathrm{j}, 1}+a_{\mathrm{j}, 2}\right)} \rightarrow \mathbb{Z}
$$

satisfying the following properties.

(1) Suppose $\left[L_{\mathrm{j}}^{\#}: L_{\mathrm{j}}\right]=d_{\mathrm{j}}^{2}$. Under the canonical embedding

$$
L_{\mathrm{j}}^{\oplus\left(a_{\mathrm{j}, 1}+a_{\mathrm{j}, 2}\right)} \hookrightarrow L_{\mathrm{j}, \text { aux }}:=L_{\mathrm{j}}^{\oplus a_{\mathrm{j}, 1}} \oplus\left(L_{\mathrm{j}}^{\#}\right)^{\oplus a_{\mathrm{j}, 2}}
$$


induced by $L_{\mathrm{j}} \hookrightarrow L_{\mathrm{j}}^{\#}$, the alternating pairing $\langle\cdot, \cdot\rangle_{\mathrm{j}} \otimes(\cdot, \cdot)_{\mathrm{j}}$ on

$$
L_{\mathrm{j}}^{\oplus\left(a_{\mathrm{j}, 1}+a_{\mathrm{j}, 2}\right)} \cong L_{\mathrm{j}} \underset{\mathbb{Z}}{\otimes \mathbb{Z}^{\oplus\left(a_{\mathrm{j}, 1}+a_{\mathrm{j}, 2}\right)}}
$$

extends to an alternating pairing $\langle\cdot, \cdot\rangle_{\mathrm{j}, \text { aux }}$ on $L_{\mathrm{j} \text {,aux }}$ valued in $\mathbb{Z}(1)$ that is self-dual at $p$ in the sense that $p \nmid\left[L_{\mathrm{j}, \text { aux }}^{\#}: L_{\mathrm{j} \text {,aux }}\right]$.

(2) Let $W$ be a (relative) abelian scheme over an algebraic stack $S$, and let $\lambda_{W}$ : $W \rightarrow W^{\vee}$ be a polarization such that $\operatorname{deg}\left(\lambda_{W}\right)=d_{\mathrm{j}}^{2}$. Let

$$
W_{\text {aux }}^{\Delta}:=W^{\times\left(a_{\mathrm{j}, 1}+a_{\mathrm{j}, 2}\right)}
$$

and

$$
W_{\mathrm{aux}}^{\nabla}:=W^{\times a_{\mathrm{j}, 1}} \underset{S}{\times}\left(W^{\vee}\right)^{\times a_{\mathrm{j}, 2}},
$$

which are fiber products over $S$; and let

$$
f:=\operatorname{Id}_{W}^{\times a_{\mathrm{j}, 1}} \underset{S}{\times} \lambda_{W}^{\times a_{\mathrm{j}, 2}}: W_{\mathrm{aux}}^{\Delta} \rightarrow W_{\mathrm{aux}}^{\nabla}
$$

Then $\lambda_{W}: W \rightarrow W^{\vee}$ and the morphism

$$
(\cdot, \cdot)_{\mathrm{j}}^{*}: \mathbb{Z}^{\oplus\left(a_{\mathrm{j}, 1}+a_{\mathrm{j}, 2}\right)} \stackrel{\sim}{\rightarrow} \mathbb{Z}^{\oplus\left(a_{\mathrm{j}, 1}+a_{\mathrm{j}, 2}\right)}
$$

canonical induced by $(\cdot, \cdot)_{\mathrm{j}}$ induce a polarization

$$
\lambda_{W, \text { aux }}^{\Delta}: W_{\text {aux }}^{\Delta} \rightarrow W_{\text {aux }}^{\Delta, v}
$$

(cf. [29, Lemmas 2.5, 2.6, and 2.9, and their proofs]), and

$$
\lambda_{W, \text { aux }}^{\nabla}:=\left(f^{\vee}\right)^{-1} \circ \lambda_{W, \text { aux }}^{\Delta} \circ f: W_{\text {aux }}^{\nabla} \rightarrow W_{\text {aux }}^{\nabla, \vee}
$$

is a polarization (not just a $\mathbb{Q}^{\times}$-polarization) of degree prime to $p$. Moreover, we can arrange that $\operatorname{deg}\left(\lambda_{W, \text { aux }}^{\nabla}\right)$ depends only on $d_{\mathrm{j}}$ and the choices of $\left(a_{\mathrm{j}, 1}\right.$, $\left.a_{\mathrm{j}, 2}\right)$ and $(\cdot, \cdot)_{\mathrm{j}}$, but not on $W$ and $\lambda_{W}$.

If $p \nmid d_{\mathrm{j}}$, then we take $\left(a_{\mathrm{j}, 1}, a_{\mathrm{j}, 2}\right)=(1,0)$ and take $(\cdot, \cdot)_{\mathrm{j}}: \mathbb{Z} \times \mathbb{Z} \rightarrow \mathbb{Z}$ to be the pairing sending $(1,1)$ to 1 . Otherwise, we take $\left(a_{\mathrm{j}, 1}, a_{\mathrm{j}, 2}\right)=(4,4)$, and take $(\cdot, \cdot)_{\mathrm{j}}$ to be defined by some $2 \times 2$ matrix $\left(\begin{array}{cc}1 & x \\ t_{x} & d_{\mathrm{j}}^{2}\end{array}\right)$ over $\mathrm{M}_{4}(\mathbb{Z})$ such that ${ }^{t} x x=d_{\mathrm{j}}^{2}-1$.

Proof. The statement is obvious when $p \nmid d_{\mathrm{j}}$. Otherwise, we can arrange that $\langle\cdot, \cdot\rangle_{\mathrm{j}, \text { aux }}$ is self-dual (at every prime) by the proof of Zarhin's trick (as in [61, Section 2] and [39, IX, 1.1]), by taking $x=\left(\begin{array}{cccc}x_{1} & -x_{2} & -x_{3} & -x_{4} \\ x_{2} & x_{1} & -x_{4} & x_{3} \\ x_{3} & x_{4} & x_{1} & -x_{2} \\ x_{4} & -x_{3} & x_{2} & x_{1}\end{array}\right)$ for any integers $x_{1}$, $x_{2}, x_{3}, x_{4}$ such that $x_{1}^{2}+x_{2}^{2}+x_{3}^{2}+x_{4}^{2}=d_{\mathrm{j}}^{2}-1$, which exist by the fact (due to Lagrange) that every nonnegative integer can be written as the sum of four squares of integers. 
LEMMA 4.5. Let $\left(Z, \lambda_{Z}\right)$ be any polarized abelian scheme over a scheme $S$. Given any integer $d_{\mathrm{j}} \geqslant 1$, let us fix the choices of $\left(a_{\mathrm{j}, 1}, a_{\mathrm{j}, 2}\right)$ and $(\cdot, \cdot)_{\mathrm{j}}$ as in Lemma 4.1. Then the functor that assigns to each scheme $T$ over $S$ the set of isomorphism classes of polarized abelian schemes $\left(W, \lambda_{W}\right)$ over $T$ such that $\operatorname{deg}\left(\lambda_{W}\right)=d_{\mathrm{j}}^{2}$ and $\left(Z, \lambda_{Z}\right) \times_{S} T \cong\left(W_{\text {aux }}^{\nabla}, \lambda_{W \text {,aux }}^{\nabla}\right)$ over $T$, where $\left(W_{\text {aux }}^{\nabla}, \lambda_{W \text {,aux }}^{\nabla}\right)$ is defined by $\left(W, \lambda_{W}\right)$ as in (2) of Lemma 4.1 , is representable by a scheme finite over $S$.

Proof. By [41, Section 16], $\operatorname{deg}\left(\lambda_{Z}\right)=d_{\mathrm{j}, \text { aux }}^{2}$ for some integer $d_{\mathrm{j}, \text { aux }} \geqslant 1$. The assertion to prove is trivially true unless the construction in (2) of Lemma 4.1 assigns to each pair $\left(W, \lambda_{W}\right)$ of genus $g$ and polarization degree $d_{\mathrm{j}}^{2}$ a pair $\left(W_{\mathrm{aux}}^{\nabla}\right.$, $\left.\lambda_{W \text {,aux }}^{\nabla}\right)$ of genus $g_{\mathrm{j} \text {,aux }}=\left(a_{\mathrm{j}, 1}+a_{\mathrm{j}, 2}\right) g$ and polarization degree $d_{\mathrm{j}, \text { aux }}^{2}$. Hence it suffices to treat the universal case, which we explain as follows.

Consider the Siegel moduli $\mathcal{A}_{g, d_{\mathrm{j}}}$ (respectively $\mathcal{A}_{g_{\mathrm{j}, \text { aux }}, d_{\mathrm{j}, \text { aux }}}$ ) of genus $g$ (respectively $g_{\mathrm{j}, \text { aux }}$ ) and polarization degree $d_{\mathrm{j}}^{2}$ (respectively $d_{\mathrm{j} \text {,aux }}^{2}$ ), which is an algebraic stack separated and of finite type over $\operatorname{Spec}(\mathbb{Z})$ (see [39, VII, 4.3] or [6, Definition 1.1 and Remark 1.2]). The assignment of pairs $\left(W_{\text {aux }}^{\nabla}, \lambda_{W, \text { aux }}^{\nabla}\right)$ to pairs $\left(W, \lambda_{W}\right)$ parameterized by $\mathcal{A}_{g, d_{\mathrm{j}}}$ as in (2) of Lemma 4.1 is functorial, and defines (by universal property) a morphism

$$
\mathcal{A}_{g, d_{\mathrm{j}}} \rightarrow \mathcal{A}_{g_{\mathrm{j}, \text { aux }}, d_{\mathrm{j}, \mathrm{aux}}} .
$$

In order to prove the lemma, it suffices to show that (4.6) is finite.

Suppose $V$ is the spectrum of a discrete valuation ring $V$ with fraction field $K$. Suppose $\left(W_{K}, \lambda_{W, K}\right)$ is an object of $\mathcal{A}_{g, d_{\mathrm{j}}}(\operatorname{Spec}(K))$, and suppose the corresponding object $\left(W_{\text {aux }, K}^{\nabla}, \lambda_{W \text {,aux }, K}^{\nabla}\right)$ of $\mathcal{A}_{g_{\mathrm{j}, \text { aux }}, d_{\mathrm{j}, \text { aux }}}(\operatorname{Spec}(K))$ extends to an object $\left(W_{\text {aux }, V}^{\nabla}, \lambda_{W, \text { aux }, V}^{\nabla}\right)$ of $\mathcal{A}_{g_{\mathrm{j}, \text { aux }}, d_{\text {j,aux }}}(\operatorname{Spec}(V))$. By the semistable reduction theorem (see, for example, [30, Theorem 3.3.2.4]), up to replacing $K$ with a finite extension field and replacing $V$ accordingly, we may assume that $W_{K}$ extends to a semiabelian scheme $W_{V}$ over $\operatorname{Spec}(V)$. By the theory of Néron models (see [4]; cf. [52, IX, 1.4], [10, Ch. I, Proposition 2.7], or [30, Proposition 3.3.1.5]), the isogeny

$$
f_{K}: W_{\mathrm{aux}, K}^{\triangle}=W_{K}^{\times\left(a_{\mathrm{j}, 1}+a_{\mathrm{j}, 2}\right)} \rightarrow W_{\mathrm{aux}, K}^{\nabla}
$$

extends to an isogeny

$$
W_{V}^{\times\left(a_{\mathrm{j}, 1}+a_{\mathrm{j}, 2}\right)} \rightarrow W_{\mathrm{aux}, V}^{\nabla}
$$

and (since $a_{\mathrm{j}, 1}+a_{\mathrm{j}, 2}>0$ ) this is possible only when $W_{V}$ is an abelian scheme; also, the polarization $\lambda_{W, K}$ extends to a polarization $\lambda_{W, V}$ of $W_{V}$. Consequently, we have an object $\left(W_{V}, \lambda_{W, V}\right)$ of $\mathcal{A}_{g, d_{\mathrm{j}}}(\operatorname{Spec}(V))$, which must correspond to the unique extension $\left(W_{\text {aux }, V}^{\nabla}, \lambda_{W, \text { aux }, V}^{\nabla}\right)$ of $\left(W_{\text {aux }, K}^{\nabla}, \lambda_{W, \text { aux }, K}^{\nabla}\right)$ (up to unique isomorphism, by the theory of Néron models again, or by the separateness 
of $\mathcal{A}_{g_{\mathrm{j}, \text { aux }}, d_{\mathrm{j}, \mathrm{aux}}}$ ). Hence (4.6) is proper by the valuative criterion (and the fact that $\mathcal{A}_{g, d_{\mathrm{j}}}$ and $\mathcal{A}_{g_{\mathrm{j}, \text { aux }}, d_{\mathrm{j}, \text { aux }}}$ are separated and of finite type over $\operatorname{Spec}(\mathbb{Z})$ ).

In order to show that (4.6) is finite, it suffices to show that the induced proper morphism

$$
\mathcal{A}_{g, d_{\mathrm{j}}} \underset{\mathbb{Z}}{\otimes} \mathbb{\mathbb { Z }}\left[\frac{1}{n}\right] \rightarrow \mathcal{A}_{g_{\mathrm{j}, \mathrm{aux},}, d_{\mathrm{j}, \mathrm{aux}}} \underset{\mathbb{Z}}{\otimes} \mathbb{Z}\left[\frac{1}{n}\right]
$$

is finite for at least two integers $n$ prime to each other. For each $n \geqslant 3$, the algebraic stack $\mathcal{A}_{g_{\mathrm{j}, \text { aux }}, d_{\mathrm{j}, \text { aux }}} \otimes_{\mathbb{Z}} \mathbb{Z}\left[\frac{1}{n}\right]$ admits a finite étale cover by the quasi projective scheme $\mathcal{A}_{g_{\mathrm{j} \text {,aux }}, d_{\mathrm{j}, \text { aux }}, n}$, defined as in [43, Ch. 7], parameterizing isomorphisms $\mathbb{Z}^{\oplus 2 g_{\mathrm{j}, \text { aux }}} \stackrel{\sim}{\rightarrow} Z[n]$ for each object $\left(Z, \lambda_{Z}\right)$ of $\mathcal{A}_{g_{\mathrm{j}, \text { aux }}, d_{\mathrm{j}, \text { aux }}} \otimes_{\mathbb{Z}} \mathbb{Z}\left[\frac{1}{n}\right]$ (in order to avoid confusion with our later terminologies, we refrain from calling such isomorphisms level structures, because they are not required to respect the pairings on both sides). Similarly, the algebraic stack $\mathcal{A}_{g, d_{\mathrm{j}}} \otimes_{\mathbb{Z}} \mathbb{Z}\left[\frac{1}{n}\right]$ admits a finite étale cover by the quasiprojective scheme $\mathcal{A}_{g, d_{\mathrm{j}}, n, n}$ parameterizing two isomorphisms $\gamma_{n}: \mathbb{Z}^{\oplus 2 g} \stackrel{\sim}{\rightarrow} W[n]$ and $\gamma_{n}^{\vee}: \mathbb{Z}^{\oplus 2 g} \stackrel{\sim}{\rightarrow} W^{\vee}[n]$ for each object ( $W, \lambda_{W}$ ) of $\mathcal{A}_{g, d_{\mathrm{j}}}$ (this is even more naive-the two isomorphisms $\gamma_{n}$ and $\gamma_{n}^{\vee}$ are not required to be related to each other under $\left.\lambda_{W}\right)$. By assigning to each object $\left(W, \lambda_{W}, \gamma_{n}, \gamma_{n}^{\vee}\right)$ of $\mathcal{A}_{g, d_{\mathrm{j}}, n, n}$ the object $\left(W_{\text {aux }}^{\nabla}, \lambda_{W, \text { aux }}^{\nabla}, \gamma_{n}^{\times a_{\mathrm{j}, 1}} \times\left(\gamma_{n}^{\vee}\right)^{\times a_{\mathrm{j}, 2}}\right)$ of

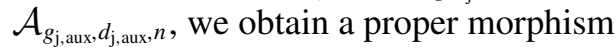

$$
\mathcal{A}_{g, d_{\mathrm{j}}, n, n} \rightarrow \mathcal{A}_{g_{\mathrm{j}, a \mathrm{au}}, d_{\mathrm{j}, \mathrm{aux}, n}}
$$

lifting (4.7). Then it suffices to show that (4.8) is finite, or rather just quasiaffine, by [14, III-1, 4.4.2].

Let $\omega_{\mathcal{A}_{g, d_{\mathrm{j}}, n, n}}$ and $\omega_{\mathcal{A}_{g_{\mathrm{j}, \text { aux }}, d_{\mathrm{j}, \text { aux }}, n}}$ denote the Hodge invertible sheaves over $\mathcal{A}_{g, d_{\mathrm{j}}, n, n}$ and $\mathcal{A}_{g_{\mathrm{j} \text {,aux }}, d_{\mathrm{j} \text {,aux }}, n}$, respectively, defined by the top exterior powers of the duals of the relative Lie algebras of the tautological abelian schemes, which are ample by [39, IX, 3.1]. By [39, IX, 2.4] and by the construction of (4.8), the pullback of a positive power of $\omega_{\mathcal{A}_{g \mathrm{j}, \text { aux } x d_{\mathrm{j}, \text { aux }, n}}}$ to $\mathcal{A}_{g, d_{\mathrm{j}}, n, n}$ is isomorphic to a positive power of $\omega_{\mathcal{A}_{g, d_{j}, n, n}}$. By [14, II, 5.1.6], these show that (4.8) is quasiaffine, as desired.

Any choices of $\left(a_{\mathrm{j}, 1}, a_{\mathrm{j}, 2}\right)$ and $(\cdot, \cdot)_{\mathrm{j}}$ as in Lemma 4.1 , for all $\mathrm{j} \in \mathrm{J}$, allow us to define the following auxiliary data.

(1) $\mathcal{O}_{\text {aux }}$ is any subring of $\mathcal{O}$ stabilized by *, with induced involution ${ }^{\star}$ aux , such that $\mathcal{O}_{\text {aux }} \otimes_{\mathbb{Z}} \mathbb{Q}$ is a semisimple algebra (finite-dimensional) over $\mathbb{Q}$; and

(2) for each $\mathrm{j} \in \mathrm{J}$, we have $L_{\mathrm{j} \text {,aux }}$ and $\langle\cdot, \cdot\rangle_{\mathrm{j} \text {, aux }}$ as in Lemma 4.1, which defines a group functor $\mathrm{G}_{\mathrm{j}, \text { aux }}$ over $\operatorname{Spec}(\mathbb{Z})$. Moreover, we have the polarization $h_{0, \mathrm{j} \text {,aux }}$ of $\left(L_{\mathrm{j}, \text { aux }},\langle\cdot, \cdot\rangle_{\mathrm{j} \text {,aux }}, \mathbb{Z}(1)\right)$ (as in [30, Definition 1.2.1.2]) canonically induced 
by $h_{0}$ by the isomorphism

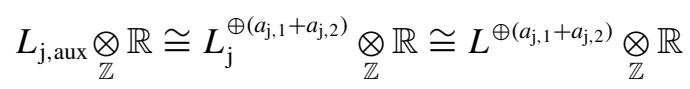

induced by (4.3), which defines an integral PEL datum

$$
\left(\mathcal{O}_{\text {aux }}, \star_{\text {aux }}, L_{\mathrm{j}, \text { aux }},\langle\cdot, \cdot\rangle_{\mathrm{j}, \text { aux }}, h_{0, \mathrm{j}, \text { aux }}\right)
$$

as in the beginning of Section 2.

Suppose moreover that, for every $\mathrm{j} \in \mathrm{J}$, the prime $p$ is good for the integral PEL datum $\left(\mathcal{O}_{\text {aux }}, \star_{\text {aux }}, L_{\mathrm{j} \text {,aux }},\langle\cdot, \cdot\rangle_{\mathrm{j} \text {,aux }}, h_{0, \mathrm{j} \text {,aux }}\right)$ as in [30, Definition 1.4.1.1], which is possible because we already know that $p \nmid\left[L_{\mathrm{j} \text {,aux }}^{\#}: L_{\mathrm{j} \text {,aux }}\right]$. Moreover, suppose that there exists a maximal order $\mathcal{O}_{\text {aux }}^{\prime}$ in $\mathcal{O}_{\text {aux }} \otimes_{\mathbb{Z}} \mathbb{Q}$ containing $\mathcal{O}_{\text {aux }}$ such that, for every $\mathrm{j} \in \mathrm{J}$, the action of $\mathcal{O}_{\text {aux }}$ on $L_{\mathrm{j} \text {,aux }}$ extends to an action of $\mathcal{O}_{\text {aux }}^{\prime}$ (see [30, Condition 1.4.3.10] and the definition of $\left\{\left(g_{\mathrm{j}}, L_{\mathrm{j}},\langle\cdot, \cdot\rangle_{\mathrm{j}}\right)\right\}_{\mathrm{j} \in \mathrm{J}}$ in Section 2). These are possible, for example, by taking $\mathcal{O}_{\text {aux }}=\mathbb{Z}$ with trivial involution ${ }^{\star}$ aux. From now on, we shall fix the auxiliary choices of $\left\{\left(a_{\mathrm{j}, 1}, a_{\mathrm{j}, 2}\right)\right\}_{\mathrm{j} \in \mathrm{J}},\left\{(\cdot, \cdot)_{\mathrm{j}}\right\}_{\mathrm{j} \in \mathrm{J}}$, and $\left\{\left(\mathcal{O}_{\text {aux }}\right.\right.$, $\left.\left.\star_{\text {aux }}, L_{\mathrm{j}, \text { aux }},\langle\cdot, \cdot\rangle_{\mathrm{j} \text {,aux }}, h_{0, \mathrm{j}, \text { aux }}\right)\right\}_{\mathrm{j} \in \mathrm{J}}$.

LEMMA 4.9. With the assumptions as above, for each $\mathrm{j} \in \mathrm{J}$, the assignment

$$
(g, r) \mapsto\left(g^{\times a_{\mathrm{j}, 1}} \times\left(r^{t} g^{-1}\right)^{\times a_{\mathrm{j}, 2}}, r\right)
$$

defines an injective homomorphism

$$
\mathrm{G}_{\mathrm{j}} \rightarrow \mathrm{G}_{\mathrm{j}, \text { aux }}
$$

of algebraic group functors over $\operatorname{Spec}(\mathbb{Z})$, which is compatible with the similitude characters and induces an injective homomorphism $\mathrm{G}_{\mathrm{j}}(\hat{\mathbb{Z}}) \rightarrow \mathrm{G}_{\mathrm{j}, \text { aux }}(\hat{\mathbb{Z}})$.

Proof. The assignment is injective because $a_{\mathrm{j}, 1}>0$, and defines a homomorphism as asserted because $\mathcal{O}_{\text {aux }}$ is a subring of $\mathcal{O}$, because ${ }^{\star}$ aux is the restriction of ${ }^{\star}$, and because $\left\langle x, r g^{-1} y\right\rangle=\langle g x, y\rangle=\left\langle x,{ }^{t} g y\right\rangle$.

LEMma 4.11. For each $\mathrm{j} \in \mathrm{J}$, the reflex field $F_{0, \mathrm{j}, \text { aux }}$ defined by the integral PEL datum $\left(\mathcal{O}_{\text {aux }}, \star_{\text {aux }}, L_{\mathrm{j}, \text { aux }},\langle\cdot, \cdot\rangle_{\mathrm{j} \text {,aux }}, h_{0, \mathrm{j} \text {,aux }}\right)$ (see [26, page 389] or [30, Definition 1.2.5.4]) is contained in $F_{0}$ (as subfields of $\mathbb{C}$ ).

Proof. In this proof, we may and we shall assume that $F_{0}$ is exactly the reflex field defined by $\left(\mathcal{O}, \star, L,\langle\cdot, \cdot\rangle, h_{0}\right)$. Since $h_{0, \mathrm{j} \text {,aux }}$ is canonically induced by $h_{0}$ by the isomorphism $L_{\mathrm{j}, \text { aux }} \otimes_{\mathbb{Z}} \mathbb{R} \cong L^{\oplus\left(a_{\mathrm{j}, 1}+a_{\mathrm{j}, 2}\right)} \otimes_{\mathbb{Z}} \mathbb{R}$ induced by (4.3), we have 
a canonical isomorphism $V_{0, \mathrm{j}, \text { aux }} \cong V_{0}^{\oplus\left(a_{\mathrm{j}, 1}+a_{\mathrm{j}, 2}\right)}$ as $\mathcal{O}_{\text {aux }} \otimes_{\mathbb{Z}} \mathbb{C}$-modules, where $V_{0}$ (respectively $V_{0, \mathrm{j}, \text { aux }}$ ) is the maximal submodule of $L \otimes_{\mathbb{Z}} \mathbb{C}$ (respectively $L_{\mathrm{j} \text {,aux }} \otimes_{\mathbb{Z}} \mathbb{C}$ ) on which $h_{0}(z)$ (respectively $\left.h_{0, \mathrm{j}, \text { aux }}(z)\right)$ acts by $1 \otimes z$. By [30, Corollary 1.2.5.6], $F_{0}$ (respectively $\left.F_{0, \mathrm{j} \text {,aux }}\right)$ is the subfield of $\mathbb{C}$ generated over $\mathbb{Q}$ by the traces $\operatorname{Tr}_{\mathbb{C}}\left(b \mid V_{0}\right)$ for $b \in \mathcal{O}$ (respectively $\operatorname{Tr}_{\mathbb{C}}\left(b \mid V_{0, \mathrm{j} \text {, aux }}\right)$ for $\left.b \in \mathcal{O}_{\text {aux }}\right)$. Hence $F_{0, \mathrm{j} \text {,aux }}$ is contained in $F_{0}$, as desired.

For each $\mathrm{j} \in \mathrm{J}$, suppose that $\mathcal{H}_{\mathrm{j} \text {,aux }}$ is an open compact subgroup of $\mathrm{G}_{\mathrm{j}, \text { aux }}\left(\hat{\mathbb{Z}}^{p}\right)$. Then we have the moduli problem $\mathrm{M}_{\mathcal{H}_{\mathrm{j} \text { aux }}}$ defined by the integral PEL datum $\left(\mathcal{O}_{\text {aux }}, \star_{\text {aux }}, L_{\mathrm{j}, \text { aux }},\langle\cdot, \cdot\rangle_{\mathrm{j} \text {,aux }}, h_{0, \mathrm{j} \text {,aux }}\right)$ and $\mathcal{H}_{\mathrm{j} \text {,aux }} \operatorname{over} \operatorname{Spec}\left(\mathcal{O}_{F_{0, \mathrm{j}, \text { aux }},(p)}\right)$, as in [30, Definition 1.4.1.4] (with $\square=\{p\}$ there). By [30, Theorem 1.4.1.11 and Corollary 7.2.3.10] again, $\mathrm{M}_{\mathcal{H}_{\mathrm{j} \text {,aux }}}$ is an algebraic stack separated, smooth, and of finite type over $\operatorname{Spec}\left(\mathcal{O}_{F_{0, \mathrm{j}, \text { aux }},(p)}\right)$, which is representable by a scheme quasiprojective (and smooth) over $\operatorname{Spec}\left(\mathcal{O}_{F_{0, \text { j,aux }},(p)}\right)$ when $\mathcal{H}_{\mathrm{j} \text {, aux }}$ is neat (our notation system here is slightly different from the one in [31, Ch. 2]: for simplicity, we dropped the superscripts ' $p$ ' in the notation of auxiliary objects).

Proposition 4.12. With assumptions as above, for each $\mathrm{j} \in \mathrm{J}$, suppose $\mathcal{H}_{\mathrm{j}, \text { aux }}$ is an open compact subgroup of $\mathrm{G}_{\mathrm{j}, \text { aux }}\left(\hat{\mathbb{Z}}^{p}\right)$ containing the image of $\mathcal{H}_{\mathrm{j}}$ under the homomorphism $\mathrm{G}_{\mathrm{j}}(\hat{\mathbb{Z}}) \rightarrow \mathrm{G}_{\mathrm{j}, \text { aux }}\left(\hat{\mathbb{Z}}^{p}\right)$ given by (4.10). Then there is a finite morphism

$$
\mathrm{M}_{\mathcal{H}} \rightarrow \mathrm{M}_{\mathcal{H}_{\mathrm{j}, \text { aux }}} \underset{\mathbb{Z}}{\otimes} \mathbb{Q}
$$

which is the composition of (2.1) with a morphism

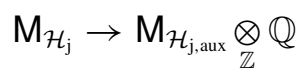

under which the pullback $\left(A_{\mathrm{j}, \text { aux }}^{\nabla}, \lambda_{\mathrm{j}, \text { aux }}^{\nabla}, i_{\mathrm{j}, \text { aux }}^{\nabla}, \alpha_{\mathcal{H}_{\mathrm{j}, \text { aux }}}^{\nabla}\right)$ of the tautological object $\left(A_{\mathrm{j}, \text { aux }}, \lambda_{\mathrm{j}, \text { aux }}, i_{\mathrm{j}, \text { aux }}, \alpha_{\mathcal{H}_{\mathrm{j}, \text { aux }}}\right)$ over $\mathrm{M}_{\mathcal{H}_{\mathrm{j} \text {,aux }}}$ to $\mathrm{M}_{\mathcal{H}_{\mathrm{j}}}$ satisfies the following properties (in terms of the tautological object $\left(A_{\mathrm{j}}, \lambda_{\mathrm{j}}, i_{\mathrm{j}}, \alpha_{\mathcal{H}_{\mathrm{j}}}\right)$ over $\left.\mathrm{M}_{\mathcal{H}_{\mathrm{j}}}\right)$.

(1) $A_{\mathrm{j}, \text { aux }}^{\nabla}$ is isomorphic to $A_{\mathrm{j}}^{\times a_{\mathrm{j}, 1}} \times_{\mathrm{M}_{\mathcal{H}_{\mathrm{j}}}}\left(A_{\mathrm{j}}^{\vee}\right)^{\times a_{\mathrm{j}, 2}}$ for the same integers $\left(a_{\mathrm{j}, 1}, a_{\mathrm{j}, 2}\right)$ as in Lemma 4.1, which is equipped with an isogeny

$$
f_{\mathrm{j}}: A_{\mathrm{j}, \text { aux }}^{\Delta}:=A_{\mathrm{j}}^{\times\left(a_{\mathrm{j}, 1}+a_{\mathrm{j}, 2}\right)} \rightarrow A_{\mathrm{j}, \text { aux }}^{\nabla}
$$

induced by $\lambda_{\mathrm{j}}: A_{\mathrm{j}} \rightarrow A_{\mathrm{j}}^{\vee}$.

(2) The polarization $\lambda_{\mathrm{j} \text {,aux }}^{\nabla}: A_{\mathrm{j} \text {,aux }}^{\nabla} \rightarrow A_{\mathrm{j} \text {,aux }}^{\nabla, \vee}$ coincides with the composition $\left(f^{\vee}\right)^{-1} \circ \lambda_{\mathrm{j}, \text { aux }}^{\Delta} \circ f\left(\right.$ as $\mathbb{Q}^{\times}$-isogenies $)$, where $\lambda_{\mathrm{j}, \text { aux }}^{\Delta}: A_{\mathrm{j}, \text { aux }}^{\Delta} \rightarrow A_{\mathrm{j}, \text { aux }}^{\Delta, \vee}$ is induced by $\lambda_{\mathrm{j}}: A_{\mathrm{j}} \rightarrow A_{\mathrm{j}}^{\vee}$ and $(\cdot, \cdot)_{\mathrm{j}}$ as in (2) of Lemma 4 .1. 
(3) The isogeny $f_{\mathrm{j}}$ above is compatible with the $\mathcal{O}_{\text {aux }}$-actions defined by the $\mathcal{O}_{\text {aux }}{ }^{-}$ structure $i_{\mathrm{j}, \text { aux }}^{\Delta}: \mathcal{O}_{\text {aux }} \rightarrow \operatorname{End}_{\mathrm{M}_{\mathcal{H}_{\mathrm{j}}}}\left(A_{\mathrm{j} \text {,aux }}^{\Delta}\right)$ induced by the restriction of $i_{\mathrm{j}}: \mathcal{O} \rightarrow$ $\operatorname{End}_{\mathrm{M}_{\mathcal{H}_{\mathrm{j}}}}\left(A_{\mathrm{j}}\right)$ to $\mathcal{O}_{\mathrm{aux}}$ and by $i_{\mathrm{j}, \text { aux }}^{\nabla}: \mathcal{O}_{\mathrm{aux}} \rightarrow \operatorname{End}_{\mathrm{M}_{\mathcal{H}_{\mathrm{j}}}}\left(A_{\mathrm{j}, \text { aux }}^{\nabla}\right)$.

(4) At each geometric point $\bar{s}$ of $\mathrm{M}_{\mathcal{H}_{\mathrm{j}}}$, the level structure $\alpha_{\mathcal{H}_{\mathrm{j}}}$ induces an $\mathcal{H}_{\mathrm{j}}$-orbit of isomorphisms

$$
\hat{\alpha}_{\bar{s}}: L_{\mathrm{j}} \underset{\mathbb{Z}}{\otimes} \hat{\mathbb{Z}} \stackrel{\sim}{\rightarrow} \mathrm{T} A_{\mathrm{j}, \bar{s}},
$$

which in turn induces an $\mathcal{H}_{\mathrm{j}, \text { aux }}$-orbit of isomorphisms

$$
\hat{\alpha}_{\bar{s}}^{\oplus\left(a_{\mathrm{j}, 1}+a_{\mathrm{j}, 2}\right)} \underset{\mathbb{\mathbb { Z }}}{\otimes} \mathbb{A}^{\infty, p}: L_{\mathrm{j}, \text { aux }} \underset{\mathbb{Z}}{\otimes} \mathbb{A}^{\infty, p} \stackrel{\sim}{\rightarrow} \mathrm{V} A_{\mathrm{j}, \text { aux }, \bar{s}}^{\nabla}
$$

(which makes sense because, by assumption, $\mathcal{H}_{\mathrm{j}}$ is mapped into $\mathcal{H}_{\mathrm{j}, \text { aux }}$ under the homomorphism $\mathrm{G}_{\mathrm{j}}(\hat{\mathbb{Z}}) \rightarrow \mathrm{G}_{\mathrm{j} \text {, aux }}\left(\hat{\mathbb{Z}}^{p}\right)$ given by (4.10)). On the other hand, the level structure $\alpha_{\mathcal{H}_{\mathrm{j}, \text { aux }}}^{\nabla}$ induces an $\mathcal{H}_{\mathrm{j}, \text { aux }}$-orbit of isomorphisms

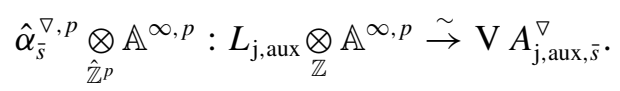

These two $\mathcal{H}_{\mathrm{j}, \text { aux }}$-orbits of isomorphisms coincide.

Suppose we replace $\mathcal{H}_{\mathrm{j}, \text { aux }}$ with an open compact subgroup $\mathcal{H}_{\mathrm{j}, \text { aux }}^{\prime}$ still containing the image of $\mathcal{H}_{\mathrm{j}}$ under the homomorphism $\mathrm{G}_{\mathrm{j}}(\hat{\mathbb{Z}}) \rightarrow \mathrm{G}_{\mathrm{j}, \text { aux }}\left(\hat{\mathbb{Z}}^{p}\right)$ given by (4.10). Then the morphism $\mathrm{M}_{\mathcal{H}_{\mathrm{j}}} \rightarrow \mathrm{M}_{\mathcal{H}_{\mathrm{j}, \text { aux }}^{\prime}} \otimes_{\mathbb{Z}} \mathbb{Q}$ thus obtained is compatible with (4.13) and the canonical morphism $\mathrm{M}_{\mathcal{H}_{\mathrm{j}, \text { aux }}^{\prime}} \otimes_{\mathbb{Z}} \mathbb{Q} \rightarrow \mathrm{M}_{\mathcal{H}_{\mathrm{j} \text {,aux }}} \otimes_{\mathbb{Z}} \mathbb{Q}$.

The morphisms (4.13), for all $\mathrm{j} \in \mathrm{J}$, induce a morphism

$$
\mathrm{M}_{\mathcal{H}} \rightarrow \prod_{\mathrm{j} \in \mathrm{J}} \mathrm{M}_{\mathcal{H}_{\mathrm{j}, \text { aux }}} \underset{\mathbb{Z}}{\otimes} \mathbb{Q}
$$

Proof. Let us first construct the morphism (4.14). Let $\left(A_{\mathrm{j}}, \lambda_{\mathrm{j}}, i_{\mathrm{j}}, \alpha_{\mathcal{H}_{\mathrm{j}}}\right)$ be the tautological object over $\mathrm{M}_{\mathcal{H}_{\mathrm{j}}}$ as in the statement of the proposition. Let $A_{\mathrm{j}, \text { aux }}^{\Delta}$, $A_{\mathrm{j}, \text { aux }}^{\nabla}, \lambda_{\mathrm{j}, \text { aux }}^{\Delta}, \lambda_{\mathrm{j} \text {,aux }}^{\nabla}$, and $f$ be defined by $\left(A_{\mathrm{j}}, \lambda_{\mathrm{j}}\right)$ as in (2) of Lemma 4.1 (with $S=\mathrm{M}_{\mathcal{H}_{\mathrm{j}}}$ there). Since $\mathcal{O}_{\text {aux }} \subset \mathcal{O}$ and since the involution ${ }^{\star}$ aux is the restriction of ${ }^{\star}$, the $\mathcal{O}$-structure $i_{\mathrm{j}}: \mathcal{O} \rightarrow \operatorname{End}_{\mathrm{M}_{\mathcal{H}_{\mathrm{j}}}}\left(A_{\mathrm{j}}\right)$ of $\left(A_{\mathrm{j}}, \lambda_{\mathrm{j}}\right)$ induces an $\mathcal{O}_{\text {aux }}$-structure

$$
i_{\mathrm{j}, \text { aux }}^{\Delta}: \mathcal{O}_{\text {aux }} \rightarrow \operatorname{End}_{\mathrm{M}_{\mathcal{H}_{\mathrm{j}}}}\left(A_{\mathrm{j}, \text { aux }}^{\Delta}\right)
$$

of $\left(A_{\mathrm{j}, \text { aux }}^{\Delta}, \lambda_{\mathrm{j} \text {,aux }}^{\Delta}\right)$, which in turn induces an $\mathcal{O}_{\text {aux }} \otimes_{\mathbb{Z}} \mathbb{Q}$-structure

$$
i_{\mathrm{j}, \text { aux }}^{\nabla}: \mathcal{O}_{\text {aux }} \underset{\mathbb{Z}}{\otimes} \mathbb{Q} \rightarrow \operatorname{End}_{\mathrm{M}_{\mathcal{H}_{\mathrm{j}}}}\left(A_{\mathrm{j}, \text { aux }}^{\nabla}\right) \underset{\mathbb{Z}}{\otimes} \mathbb{Q}
$$


of $\left(A_{\mathrm{j}, \text { aux }}^{\nabla}, \lambda_{\mathrm{j}, \text { aux }}^{\nabla}\right)$ as in [30, Definition 1.3.3.1] by

$$
i_{\mathrm{j}, \text { aux }}^{\nabla}(b):=f \circ i_{\mathrm{j}, \text { aux }}^{\Delta}(b) \circ f^{-1}
$$

for each $b \in \mathcal{O}_{\text {aux. }}$

At each geometric point $\bar{s}$ of $\mathrm{M}_{\mathcal{H}, \mathrm{j}}$, the level structure $\alpha_{\mathcal{H}_{\mathrm{j}}}$ lifts to some $\mathcal{O} \otimes_{\mathbb{Z}} \hat{\mathbb{Z}}$ equivariant isomorphism

$$
\hat{\alpha}_{\bar{s}}: L_{\mathrm{j}} \underset{\mathbb{Z}}{\otimes} \hat{\mathbb{Z}} \stackrel{\sim}{\rightarrow} \mathrm{T} A_{\mathrm{j}, \bar{s}},
$$

which induces an $\mathcal{O}_{\text {aux }} \otimes_{\mathbb{Z}} \hat{\mathbb{Z}}$-equivariant isomorphism

$$
\hat{\alpha}_{\bar{s}}^{\Delta}:=\hat{\alpha}_{\bar{s}}^{\oplus\left(a_{\mathrm{j}, 1}+a_{\mathrm{j}, 2}\right)}:\left(L_{\mathrm{j}}^{\oplus\left(a_{\mathrm{j}, 1}+a_{\mathrm{j}, 2}\right)}\right) \underset{\mathbb{Z}}{\otimes} \hat{\mathbb{Z}} \stackrel{\sim}{\rightarrow} \mathrm{T} A_{\mathrm{j}, \text { aux }, \bar{s}}^{\Delta}
$$

and an $\mathcal{O}_{\text {aux }} \otimes_{\mathbb{Z}} \mathbb{A}^{\infty}$-equivariant isomorphism

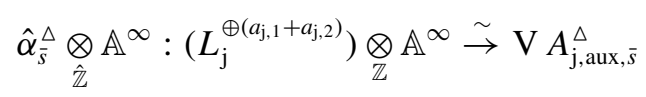

(all matching similitudes, implicitly). By [30, Lemma 1.3.5.2], under the isomorphism $\hat{\alpha}_{\bar{s}} \otimes_{\hat{\mathbb{Z}}} \mathbb{A}^{\infty}: L_{\mathrm{j}} \otimes_{\mathbb{Z}} \mathbb{A}^{\infty} \stackrel{\sim}{\rightarrow} \mathrm{V} A_{\mathrm{j}, \bar{s}}$, the polarization (as an $\mathcal{O}$ equivariant isogeny) $\lambda_{\mathrm{j}, \bar{s}}: A_{\mathrm{j}, \bar{s}} \rightarrow A_{\mathrm{j}, \bar{s}}^{\vee}$ corresponds to the open compact subgroup $L_{\mathrm{j}}^{\#} \otimes_{\mathbb{Z}} \hat{\mathbb{Z}}$ of $L_{\mathrm{j}} \otimes_{\mathbb{Z}} \mathbb{A}^{\infty}$. Hence the restriction of $\hat{\alpha}_{\bar{s}}^{\Delta} \otimes_{\hat{\mathbb{Z}}} \mathbb{A}^{\infty}$ induces an $\mathcal{O}_{\text {aux }} \otimes_{\mathbb{Z}} \hat{\mathbb{Z}}$ equivariant isomorphism

$$
\hat{\alpha}_{\bar{s}}^{\nabla}: L_{\mathrm{j}, \text { aux }} \underset{\mathbb{Z}}{\otimes} \hat{\mathbb{Z}} \stackrel{\sim}{\rightarrow} \mathrm{T} A_{\mathrm{j}, \text { aux }, \bar{s}}^{\nabla} \cdot
$$

Since the choices of $\bar{s}$ and $\hat{\alpha}_{\bar{s}}$ are arbitrary, by [30, Lemma 1.3.5.2] again, the $\mathcal{O}_{\text {aux }} \otimes_{\mathbb{Z}} \mathbb{Q}$-structure $i_{\mathrm{j} \text {,aux }}^{\nabla}$ above induces an $\mathcal{O}_{\text {aux }}$-structure

$$
i_{\mathrm{j}, \text { aux }}^{\nabla}: \mathcal{O}_{\mathrm{aux}} \rightarrow \operatorname{End}_{\mathrm{M}_{\mathcal{H}_{\mathrm{j}}}}\left(A_{\mathrm{j}, \text { aux }}^{\nabla}\right)
$$

of $\left(A_{\mathrm{j} \text {,aux }}^{\nabla}, \lambda_{\mathrm{j}, \text { aux }}^{\nabla}\right)$. Moreover, by forgetting the factor at $p$, the $\hat{\alpha}_{\bar{s}}^{\nabla}$ above induces an $\mathcal{O}_{\text {aux }} \otimes_{\mathbb{Z}} \hat{\mathbb{Z}}^{p}$-equivariant isomorphism

$$
\hat{\alpha}_{\bar{s}}^{\nabla, p}: L_{\mathrm{j}, \text { aux }} \underset{\mathbb{Z}}{\otimes} \hat{\mathbb{Z}}^{p} \stackrel{\sim}{\rightarrow} \mathrm{T}^{p} A_{\mathrm{j}, \text { aux }, \bar{s}}^{\nabla} .
$$

Since the $\mathcal{H}_{\mathrm{j}}$-orbit of $\hat{\alpha}_{\bar{s}}$ is $\pi_{1}\left(\mathrm{M}_{\mathcal{H}_{\mathrm{j}}}, \bar{s}\right)$-invariant, and since $\mathcal{H}_{\mathrm{j}}$ is mapped to a subgroup of $\mathcal{H}_{\mathrm{j} \text {, aux }}$ under the homomorphism $\mathrm{G}_{\mathrm{j}}(\hat{\mathbb{Z}}) \rightarrow \mathrm{G}_{\mathrm{j}, \text { aux }}\left(\hat{\mathbb{Z}}^{p}\right)$ given by (4.10), the $\mathcal{H}_{\mathrm{j} \text {,aux }}$-orbit $\left[\hat{\alpha}_{\bar{s}}^{\nabla, p}\right]_{\mathcal{H}_{\mathrm{j} \text {,aux }}}$ of $\hat{\alpha}_{\bar{s}}^{\nabla, p}$ is $\pi_{1}\left(\mathrm{M}_{\mathcal{H}_{\mathrm{j}}}, \bar{s}\right)$-invariant. By [30, Proposition 1.4.3.4], the tuple $\left(A_{\mathrm{j}, \text { aux }}^{\nabla}, \lambda_{\mathrm{j}, \text { aux }}^{\nabla}, i_{\mathrm{j}, \text { aux }}^{\nabla},\left[\hat{\alpha}_{\bar{s}}^{\nabla, p}\right]_{\mathcal{H}_{\mathrm{j}, \text { aux }}}\right)$ defines an object

$$
\left(A_{\mathrm{j}, \text { aux }}^{\nabla}, \lambda_{\mathrm{j}, \text { aux }}^{\nabla}, i_{\mathrm{j}, \text { aux }}^{\nabla}, \alpha_{\mathcal{H}_{\mathrm{j}, \text { aux }}}^{\nabla}\right)
$$


of $M_{\mathcal{H}_{\mathrm{j}, \text { aux }}}$ over $\mathrm{M}_{\mathcal{H}_{\mathrm{j}}}$, which satisfies the properties described in the proposition by its very construction.

We would like to show that $\underline{\mathrm{Lie}}_{A_{\mathrm{j}, \text { aux }}^{\nabla} / \mathrm{M}_{\mathcal{H}_{\mathrm{j}}}}$ with its $\mathcal{O} \otimes_{\mathbb{Z}} \mathbb{Q}$-module structure given by $i_{\mathrm{j}, \text { aux }}^{\nabla}$ satisfies the determinantal condition given by

$$
\left(L_{\mathrm{j}, \text { aux }} \underset{\mathbb{Z}}{\otimes} \mathbb{R},\langle\cdot, \cdot\rangle_{\mathrm{j}, \text { aux }}, h_{0, \mathrm{j}, \text { aux }}\right)
$$

as in [30, Definition 1.3.4.1]. Since this condition is closed by definition, and is open in characteristic zero by [30, Lemma 1.2.5.11], it suffices to verify it at each $\mathbb{C}$-point $t$ of $\mathrm{M}_{\mathcal{H}_{\mathrm{j}}}$. Let $\left(A_{\mathrm{j}, t}, \lambda_{\mathrm{j}, t}, i_{\mathrm{j}, t}\right)$ and $\left(A_{\mathrm{j}, \text { aux }, t}^{\nabla}, \lambda_{\mathrm{j}, \text { aux }, t}^{\nabla}, i_{\mathrm{j}, \text { aux }, t}^{\nabla}\right)$ denote the respective pullbacks of $\left(A_{\mathrm{j}}, \lambda_{\mathrm{j}}, i_{\mathrm{j}}\right)$ and $\left(A_{\mathrm{j}, \text { aux }}^{\nabla}, \lambda_{\mathrm{j}, \text { aux }}^{\nabla}, i_{\mathrm{j} \text {,aux }}^{\nabla}\right)$ to such a $\mathbb{C}$-point $t$. By [30, Lemma 1.2.5.11] again, since $\underline{\mathrm{Lie}}_{A_{\mathrm{j}} / \mathrm{M}_{\mathcal{H}_{\mathrm{j}}}}$ with its $\mathcal{O} \otimes_{\mathbb{Z}} \mathbb{Q}$-module structure given by $i$ satisfies the determinantal condition given by

$$
\left(L_{\mathrm{j}} \underset{\mathbb{Z}}{\otimes} \mathbb{R},\langle\cdot, \cdot\rangle_{\mathrm{j}}, h_{0}\right) \cong\left(L \underset{\mathbb{Z}}{\otimes} \mathbb{R},\langle\cdot, \cdot\rangle, h_{0}\right),
$$

we have $\operatorname{Lie}_{A_{\mathrm{j}, t}} \cong V_{0}$ as $\mathcal{O} \otimes_{\mathbb{Z}} \mathbb{C}$-modules, and it suffices to note that

$$
\operatorname{Lie}_{A_{\mathrm{j}, \text { aux }, t}^{\nabla}} \cong \operatorname{Lie}_{A_{\mathrm{j}, t}}^{\oplus a_{\mathrm{j}, 1}} \oplus \operatorname{Lie}_{A_{\mathrm{j}, t}^{\mathrm{v}}}^{\oplus a_{\mathrm{j}, 2}} \cong V_{0}^{\oplus\left(a_{\mathrm{j}, 1}+a_{\mathrm{j}, 2}\right)} \cong V_{0, \mathrm{j}, \text { aux }}
$$

as $\mathcal{O}_{\text {aux }} \otimes_{\mathbb{Z}} \mathbb{C}$-modules (cf. the proof of Lemma 4.11).

Thus we have obtained the desired (4.14) by the moduli interpretation of $\mathrm{M}_{\mathcal{H}_{\mathrm{j}, \text { aux }} \text {, }}$, whose precomposition with (2.1) gives the desired (4.13). The morphism (4.14) between algebraic stacks is schematic and finite by Lemma 4.5 (for the abelian schemes and polarizations), by [30, Proposition 1.3.3.7] (for the endomorphism structures), and by the fact that the level structures are defined by isomorphisms between finite étale group schemes; hence so is the morphism (4.13).

LEMMA 4.16. With assumptions as above, suppose the image $\mathcal{H}^{p}$ of $\mathcal{H}$ under the canonical homomorphism $\mathrm{G}(\hat{\mathbb{Z}}) \rightarrow \mathrm{G}\left(\hat{\mathbb{Z}}^{p}\right)$ is neat (which means, a fortiori, that $\mathcal{H}$ is also neat). Then, for each $\mathrm{j} \in \mathrm{J}$, there exists a neat open compact subgroup $\mathcal{H}_{\mathrm{j}, \text { aux }}$ of $\mathrm{G}_{\mathrm{j}, \text { aux }}\left(\hat{\mathbb{Z}}^{p}\right)$ such that $\mathcal{H}_{\mathrm{j}}$ is mapped to a subgroup of $\mathcal{H}_{\mathrm{j}, \text { aux }}$ under the homomorphism $\mathrm{G}_{\mathrm{j}}(\hat{\mathbb{Z}}) \rightarrow \mathrm{G}_{\mathrm{j}, \text { aux }}\left(\hat{\mathbb{Z}}^{p}\right)$ given by (4.10).

Proof. Since $\mathcal{H}_{\mathrm{j}}$ is the preimage of $g_{\mathrm{j}}^{-1} \mathcal{H} g_{\mathrm{j}}$ under the canonical isomorphism $\mathrm{G}_{\mathrm{j}}\left(\mathbb{A}^{\infty}\right) \cong \mathrm{G}\left(\mathbb{A}^{\infty}\right)$, the assumption implies that, for each $\mathrm{j} \in \mathrm{J}$, the image $\mathcal{H}_{\mathrm{j}}^{p}$ of $\mathcal{H}_{\mathrm{j}}$ under the canonical homomorphism $\mathrm{G}_{\mathrm{j}}(\hat{\mathbb{Z}}) \rightarrow \mathrm{G}_{\mathrm{j}}\left(\hat{\mathbb{Z}}^{p}\right)$ is also neat. Let $n_{0} \geqslant 3$ be an integer prime to $p$ such that $\mathcal{H}_{\mathrm{j}}^{p}$ contains

$$
\mathcal{U}_{\mathrm{j}}^{p}\left(n_{0}\right):=\operatorname{ker}\left(\mathrm{G}_{\mathrm{j}}\left(\hat{\mathbb{Z}}^{p}\right) \rightarrow \mathrm{G}_{\mathrm{j}}\left(\hat{\mathbb{Z}}^{p} / n_{0} \hat{\mathbb{Z}}^{p}\right)=\mathrm{G}_{\mathrm{j}}\left(\mathbb{Z} / n_{0} \mathbb{Z}\right)\right),
$$


and let $\mathcal{H}_{\mathrm{j} \text {,aux }}$ be generated by

$$
\mathcal{U}_{\mathrm{j}, \text { aux }}\left(n_{0}\right):=\operatorname{ker}\left(\mathrm{G}_{\mathrm{j}, \text { aux }}\left(\hat{\mathbb{Z}}^{p}\right) \rightarrow \mathrm{G}_{\mathrm{j}, \text { aux }}\left(\hat{\mathbb{Z}}^{p} / n_{0} \hat{\mathbb{Z}}^{p}\right)=\mathrm{G}_{\mathrm{j}, \text { aux }}\left(\mathbb{Z} / n_{0} \mathbb{Z}\right)\right)
$$

and the image of $\mathcal{H}_{\mathrm{j}}^{p}$ under the injective homomorphism $\mathrm{G}_{\mathrm{j}}\left(\hat{\mathbb{Z}}^{p}\right) \rightarrow \mathrm{G}_{\mathrm{j}, \text { aux }}\left(\hat{\mathbb{Z}}^{p}\right)$ given by (4.10). Then every element of $\mathcal{H}_{\mathrm{j}, \text { aux }}$ is congruent modulo $n_{0}$ to the image of some element of $\mathcal{H}_{\mathrm{j}}^{p}$, which is neat as explained above; and so $\mathcal{H}_{\mathrm{j} \text {, aux }}$ is also neat, by definition (see [49, Section 0.6] or [30, Definition 1.4.1.8]), and by Serre's lemma that no nontrivial root of unity can be congruent to 1 modulo $n$ if $n \geqslant 3$.

\section{Auxiliary choices of toroidal and minimal compactifications}

Let us fix a choice of $\mathrm{j} \in \mathrm{J}$ in the following paragraphs. Each symplectic admissible filtration $Z_{\mathrm{j}}=\left\{Z_{\mathrm{j},-i}\right\}_{i}$ of $L_{\mathrm{j}} \otimes_{\mathbb{Z}} \hat{\mathbb{Z}}$ (see [30, Section 1.2.6]) induces a symplectic admissible filtration $Z_{\mathrm{j}, \text { aux }}=\left\{Z_{\mathrm{j}, \text { aux },-i}\right\}_{i}$ of $L_{\mathrm{j} \text {,aux }} \otimes_{\mathbb{Z}} \hat{\mathbb{Z}}^{p}$ by setting

$$
\mathrm{Z}_{\mathrm{j}, \text { aux },-i}:=\left(\left(\mathrm{Z}_{\mathrm{j},-i}^{\oplus\left(a_{\mathrm{j}, 1}+a_{\mathrm{j}, 2}\right)}\right) \underset{\hat{\mathbb{Z}}}{\otimes} \mathbb{A}^{\infty, p}\right) \cap\left(L_{\mathrm{j}, \text { aux }} \underset{\mathbb{Z}}{\otimes} \hat{\mathbb{Z}}^{p}\right)
$$

as submodules of $L_{\mathrm{j}, \text { aux }} \otimes_{\mathbb{Z}} \mathbb{A}^{\infty, p}$. If $Z_{\mathrm{j}}$ is fully symplectic (see [30, Definition 5.2.7.1]), which means $Z_{j}$ extends to a symplectic filtration $Z_{j, \mathbb{A}}=\left\{Z_{j,-i, \mathbb{A}}\right\}_{i}$ of $L_{\mathrm{j}} \otimes_{\mathbb{Z}} \mathbb{A}$, then $\mathrm{Z}_{\mathrm{j} \text {,aux }}=\left\{\mathrm{Z}_{\mathrm{j} \text {,aux, }-i}\right\}_{i}$ also extends to a filtration $\mathrm{Z}_{\mathrm{j} \text {,aux, } \mathbb{A}^{p}}=$ $\left\{Z_{\mathrm{j}, \text { aux },-i, \mathbb{A}^{p}}\right\}_{i}$ on $L_{\mathrm{j} \text {,aux }} \otimes_{\mathbb{Z}} \mathbb{A}^{p}$, by setting $\mathrm{Z}_{\mathrm{j}, \text { aux },-i, \mathbb{A}^{p}}:=\mathrm{Z}_{\mathrm{j},-i, \mathbb{A}}^{\oplus\left(a_{\mathrm{j}, 1}+a_{\mathrm{j}, 2}\right)} \otimes_{\mathbb{A}} \mathbb{A}^{p}$. These definitions are compatible with the actions of $\mathrm{G}_{\mathrm{j}}\left(\mathbb{A}^{p}\right)$ and $\mathrm{G}_{\mathrm{j}, \text { aux }}\left(\mathbb{A}^{p}\right)$ (and with the homomorphism $G_{\mathrm{j}}\left(\mathbb{A}^{p}\right) \rightarrow \mathrm{G}_{\mathrm{j}, \text { aux }}\left(\mathbb{A}^{p}\right)$ given by (4.10)), and are compatible with reductions modulo $n$ for any integer $n \geqslant 1$ prime to $p$. Thus, there is a welldefined assignment

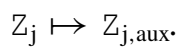

If $\Phi_{\mathrm{j}}=\left(X_{\mathrm{j}}, Y_{\mathrm{j}}, \phi_{\mathrm{j}}, \varphi_{\mathrm{j},-2}, \varphi_{\mathrm{j}, 0}\right)$ is a torus argument of $\mathrm{Z}_{\mathrm{j}}$ (see [30, Definition 5.4.1.3]), then we define

$$
X_{\mathrm{j}, \text { aux }}:=X_{\mathrm{j}}^{\oplus a_{\mathrm{j}, 1}} \oplus Y_{\mathrm{j}}^{\oplus a_{\mathrm{j}, 2}}
$$

and

$$
Y_{\mathrm{j}, \text { aux }}:=Y_{\mathrm{j}}^{\oplus a_{\mathrm{j}, 1}} \oplus X_{\mathrm{j}}^{\oplus a_{\mathrm{j}, 2}} .
$$

LEMMA 5.3. With the setting as above, there exist canonically induced morphisms

$$
\begin{gathered}
\phi_{\mathrm{j}, \text { aux }}: Y_{\mathrm{j}, \text { aux }} \hookrightarrow X_{\mathrm{j}, \text { aux }}, \\
\varphi_{\mathrm{j}, \text { aux },-2}: \mathrm{Gr}_{-2}^{\mathrm{Z}_{\mathrm{j}, \text { aux }}} \stackrel{\sim}{\rightarrow} \operatorname{Hom}_{\hat{\mathbb{Z}}}\left(X_{\mathrm{j}, \text { aux }} \underset{\mathbb{Z}}{\otimes} \hat{\mathbb{Z}}^{p}, \hat{\mathbb{Z}}^{p}(1)\right),
\end{gathered}
$$


and

$$
\varphi_{\mathrm{j}, \text { aux }, 0}: \mathrm{Gr}_{0}^{\mathrm{z}_{\mathrm{j}, \text { aux }}} \stackrel{\sim}{\rightarrow} Y_{\mathrm{j}, \text { aux }} \underset{\mathbb{Z}}{\otimes} \hat{\mathbb{Z}}^{p}
$$

making

$$
\Phi_{\mathrm{j}, \text { aux }}:=\left(X_{\mathrm{j}, \text { aux }}, Y_{\mathrm{j}, \text { aux }}, \phi_{\mathrm{j}, \text { aux }}, \varphi_{\mathrm{j}, \text { aux },-2}, \varphi_{\mathrm{j}, \text { aux }, 0}\right)
$$

a torus argument of $\mathrm{Z}_{\mathrm{j}, \text { aux }}$, and making the diagrams

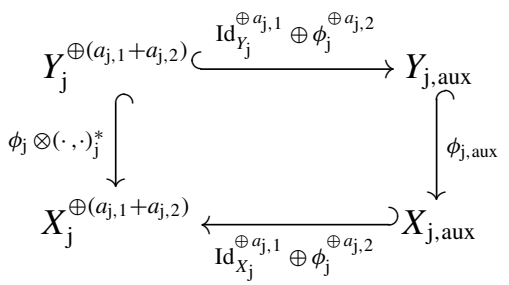

$$
\begin{aligned}
& \left(\mathrm{Gr}_{-2}^{\mathrm{Z}_{\mathrm{j}}}\right)^{\oplus\left(a_{\mathrm{j}, 1}+a_{\mathrm{j}, 2}\right)} \longrightarrow \mathrm{Gr}_{-2}^{\mathrm{Z}_{\mathrm{j}, \text { aux }}} \\
& \varphi_{\mathrm{j},-2}^{\oplus\left(a_{\mathrm{j}, 1}+a_{\mathrm{j}, 2}\right)} \downarrow_{2} \quad 2 \downarrow \varphi_{\mathrm{j}, \text { aux },-2}
\end{aligned}
$$

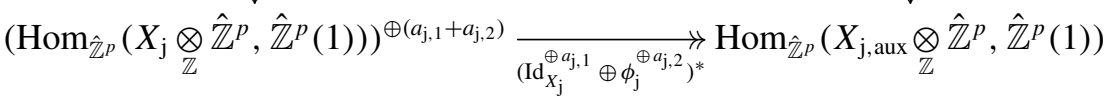

and

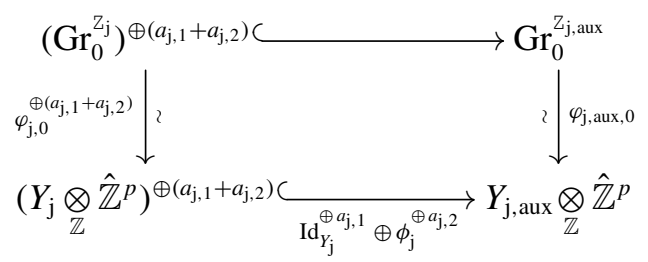

commutative, where $(\cdot, \cdot)_{\mathrm{j}}^{*}$ is canonically induced by $(\cdot, \cdot)_{\mathrm{j}}$ as in Lemma 4.1.

Proof. These follow from Lemma 4.1 and from the construction of the filtration $\mathrm{Z}_{\mathrm{j}, \text { aux },-i}$ in (5.1) above.

Let us take any splitting $\delta_{\mathrm{j} \text {,aux }}$ of $\mathrm{Z}_{\mathrm{j} \text {,aux }}$, and abusively denote its $\mathcal{H}_{\mathrm{j} \text {,aux }}$-orbit by $\delta_{\mathcal{H}_{\mathrm{j}, \text { aux }}}$. Since $\mathcal{H}_{\mathrm{j}}$ is mapped into $\mathcal{H}_{\mathrm{j} \text {, aux }}$ under the homomorphism

$$
\mathrm{G}_{\mathrm{j}}(\hat{\mathbb{Z}}) \rightarrow \mathrm{G}_{\mathrm{j}, \text { aux }}\left(\hat{\mathbb{Z}}^{p}\right)
$$

given by (4.10), and since the above assignments are compatible with the formations of orbits, we obtain a well-defined assignment of cusp labels

$$
\left[\left(\mathrm{Z}_{\mathcal{H}_{\mathrm{j}}}, \Phi_{\mathcal{H}_{\mathrm{j}}}, \delta_{\mathcal{H}_{\mathrm{j}}}\right)\right] \mapsto\left[\left(\mathrm{Z}_{\mathcal{H}_{\mathrm{j}, \text { aux }}}, \Phi_{\mathcal{H}_{\mathrm{j}, \text { aux }}}, \delta_{\mathcal{H}_{\mathrm{j}, \text { aux }}}\right)\right]
$$


Let us fix once and for all the choices of $\delta_{\mathrm{j} \text {,aux }}$ for all pairs $\left(\mathrm{Z}_{\mathrm{j}, \text { aux }}, \Phi_{\mathrm{j}, \text { aux }}\right)$, which determine the choices of $\delta_{\mathcal{H}_{\mathrm{j}, \text { aux }}}$ for all pairs $\left(\mathrm{Z}_{\mathcal{H}_{\mathrm{j} \text {,aux }}}, \Phi_{\mathcal{H}_{\mathrm{j}, \text { aux }}}\right)$. Then (5.7) is induced by an assignment of representatives of cusp labels

$$
\left(\mathrm{Z}_{\mathcal{H}_{\mathrm{j}}}, \Phi_{\mathcal{H}_{\mathrm{j}}}, \delta_{\mathcal{H}_{\mathrm{j}}}\right) \mapsto\left(\mathrm{Z}_{\mathcal{H}_{\mathrm{j}, \text { aux }}}, \Phi_{\mathcal{H}_{\mathrm{j}, \text { aux }}}, \delta_{\mathcal{H}_{\mathrm{j} \text {,aux }}}\right) .
$$

Moreover, by Lemma 5.3, tensor products with the symmetric bilinear pairing $(\cdot, \cdot)_{\mathrm{j}}$ in Lemma 4.1 induce an embedding

$$
\left(\mathbf{S}_{\Phi_{\mathcal{H}_{\mathrm{j}}}}\right)_{\mathbb{Q}}^{\vee} \hookrightarrow\left(\mathbf{S}_{\Phi_{\mathcal{H}_{\mathrm{j}, \text { aux }}}}\right)_{\mathbb{Q}}^{\vee}: y \mapsto y \otimes(\cdot, \cdot)_{\mathrm{j}}
$$

(by forgetting the compatibility of the pairings with $\mathcal{O}$, but retaining only the compatibility of the pairings with $\mathcal{O}_{\text {aux }}$. Since $(\cdot, \cdot)_{\mathrm{j}}$ is positive definite, the embedding

$$
\left(\mathbf{S}_{\Phi_{\mathcal{H}_{\mathrm{j}}}}\right)_{\mathbb{R}}^{\vee} \hookrightarrow\left(\mathbf{S}_{\Phi_{\mathcal{H}_{\mathrm{j} \text {,aux }}}}\right)_{\mathbb{R}}^{\vee}
$$

induced by (5.9) maps $\mathbf{P}_{\Phi_{\mathcal{H}_{\mathrm{j}}}}$ (respectively $\mathbf{P}_{\Phi_{\mathcal{H}_{\mathrm{j}}}^{+}}$) to $\mathbf{P}_{\Phi_{\mathcal{H}_{\mathrm{j}, \text { aux }}}}$ (respectively $\mathbf{P}_{\Phi_{\mathcal{H}_{\mathrm{j}, \text { aux }}}^{+}}$). The dual of (5.9) gives a surjection

$$
\left(\mathbf{S}_{\Phi_{\mathcal{H}_{\mathrm{j}, \text { aux }}}}\right)_{\mathbb{Q}}:=\mathbf{S}_{\Phi_{\mathcal{H}_{\mathrm{j}, \text { aux }}}} \underset{\mathbb{Z}}{\otimes} \mathbb{Q} \rightarrow\left(\mathbf{S}_{\Phi_{\mathcal{H}_{\mathrm{j}}}}\right)_{\mathbb{Q}},
$$

which induces a homomorphism

$$
\mathbf{S}_{\Phi_{\mathcal{H}_{\mathrm{j}, \text { aux }}}} \rightarrow \mathbf{S}_{\Phi_{\mathcal{H}_{\mathrm{j}}}}
$$

The composition of (3.8) and (5.8) gives an assignment

$$
\left(\mathrm{Z}_{\mathcal{H}}, \Phi_{\mathcal{H}}, \delta_{\mathcal{H}}\right) \mapsto\left(\mathrm{Z}_{\mathcal{H}_{\mathrm{j} \text {,aux }}}, \Phi_{\mathcal{H}_{\mathrm{j} \text {,aux }}}, \delta_{\mathcal{H}_{\mathrm{j}, \text { aux }}}\right)
$$

of representatives of cusp labels, which induces the assignment

$$
\left[\left(\mathrm{Z}_{\mathcal{H}}, \Phi_{\mathcal{H}}, \delta_{\mathcal{H}}\right)\right] \mapsto\left[\left(\mathrm{Z}_{\mathcal{H}_{\mathrm{j} \text {,aux }}}, \Phi_{\mathcal{H}_{\mathrm{j} \text {,aux }}}, \delta_{\mathcal{H}_{\mathrm{j}, \text { aux }}}\right)\right]
$$

of cusp labels, which is the composition of (3.7) and (5.7). Suppose $\left(Z_{\mathcal{H}}, \Phi_{\mathcal{H}}\right.$, $\left.\delta_{\mathcal{H}}\right)$ is mapped to $\left(Z_{\mathcal{H}_{\mathrm{j}}}, \Phi_{\mathcal{H}_{\mathrm{j}}}, \delta_{\mathcal{H}_{\mathrm{j}}}\right)$ in (3.8). By pre- or postcomposition of the maps (5.9), (5.10), (5.11), and (5.12) with (3.11), (3.12), (3.10), and (3.9), respectively, we obtain the maps

$$
\begin{aligned}
& \left(\mathbf{S}_{\Phi_{\mathcal{H}}}\right)_{\mathbb{Q}}^{\vee} \hookrightarrow\left(\mathbf{S}_{\Phi_{\mathcal{H}_{\mathrm{j}} \text {,aux }}}\right)_{\mathbb{Q}}^{\vee}, \\
& \left(\mathbf{S}_{\Phi_{\mathcal{H}}}\right)_{\mathbb{R}}^{\vee} \hookrightarrow\left(\mathbf{S}_{\Phi_{\mathcal{H}_{\mathrm{j}} \text {,aux }}}\right)_{\mathbb{R}}^{\vee}, \\
& \left(\mathbf{S}_{\Phi_{\mathcal{H}_{\mathrm{j}, \text { aux }}}}\right)_{\mathbb{Q}} \rightarrow\left(\mathbf{S}_{\Phi_{\mathcal{H}}}\right)_{\mathbb{Q}},
\end{aligned}
$$

and

$$
\mathbf{S}_{\Phi_{\mathcal{H}_{\mathrm{j}, \mathrm{aux}}}} \rightarrow \mathbf{S}_{\Phi_{\mathcal{H}}}
$$


which are compatible with each other, where (5.16) maps $\mathbf{P}_{\Phi_{\mathcal{H}}}$ (respectively $\mathbf{P}_{\Phi_{\mathcal{H}}}^{+}$) to $\mathbf{P}_{\Phi_{\mathcal{H}_{\mathrm{j} \text {,aux }}}}$ (respectively $\mathbf{P}_{\Phi_{\mathcal{H}_{\mathrm{j}, \text { aux }}}^{+}}$). By taking products over the indices $\mathrm{j} \in \mathrm{J}$, these maps (5.15), (5.16), (5.17), and (5.18) induce the maps

$$
\begin{gathered}
\left(\mathbf{S}_{\Phi_{\mathcal{H}}}\right)_{\mathbb{Q}}^{\vee} \hookrightarrow \prod_{\mathrm{j} \in \mathrm{J}}\left(\mathbf{S}_{\Phi_{\mathcal{H}_{\mathrm{j}, \mathrm{aux}}}}\right)_{\mathbb{Q}}^{\vee}, \\
\left(\mathbf{S}_{\Phi_{\mathcal{H}}}\right)_{\mathbb{R}}^{\vee} \hookrightarrow \prod_{\mathrm{j} \in \mathrm{J}}\left(\mathbf{S}_{\Phi_{\mathcal{H}_{\mathrm{j}, \mathrm{aux}}}}\right)_{\mathbb{R}}^{\vee}, \\
\prod_{\mathrm{j} \in \mathrm{J}}\left(\mathbf{S}_{\Phi_{\mathcal{H}_{\mathrm{j}, \text { aux }}}}\right)_{\mathbb{Q}} \rightarrow\left(\mathbf{S}_{\Phi_{\mathcal{H}}}\right)_{\mathbb{Q}},
\end{gathered}
$$

and

$$
\prod_{\mathrm{j} \in \mathrm{J}} \mathbf{S}_{\Phi_{\mathcal{H}_{\mathrm{j}, \mathrm{aux}}}} \rightarrow \mathbf{S}_{\Phi_{\mathcal{H}}}
$$

respectively, which are compatible with each other, where (5.20) maps $\mathbf{P}_{\Phi_{\mathcal{H}}}$ (respectively $\mathbf{P}_{\Phi_{\mathcal{H}}}^{+}$) to $\prod_{\mathrm{j} \in \mathrm{J}} \mathbf{P}_{\Phi_{\mathcal{H}_{\mathrm{j}, \text { aux }}}}$ (respectively $\prod_{\mathrm{j} \in \mathrm{J}} \mathbf{P}_{\Phi_{\mathcal{H}_{\mathrm{j}, \mathrm{aux}}}}^{+}$). Given a nondegenerate rational polyhedral cone $\sigma_{\mathrm{j}, \text { aux }}$ in $\mathbf{P}_{\Phi_{\mathcal{H}_{\mathrm{j}, \text { aux }}}}$ (respectively $\mathbf{P}_{\Phi_{\mathcal{H}_{\mathrm{j}} \text {,aux }}}^{+}$) for each $\mathrm{j} \in \mathrm{J}$, the pullback of $\prod_{\mathrm{j} \in \mathrm{J}} \sigma_{\mathrm{j} \text {, aux }}$ under (5.20) is either empty or a nondegenerate rational polyhedral cone $\sigma$ in $\mathbf{P}_{\Phi_{\mathcal{H}}}$ (respectively $\mathbf{P}_{\Phi_{\mathcal{H}}}^{+}$) (however, $\sigma$ might not be smooth even when $\sigma_{\mathrm{j} \text {,aux }}$ is for all $\mathrm{j} \in \mathrm{J}$ ).

Definition 5.23. Suppose $\mathrm{j} \in \mathrm{J}$. Let $\Sigma_{\mathrm{j}}$ (respectively $\Sigma_{\mathrm{j} \text {,aux }}$ ) be a compatible choice of admissible smooth rational polyhedral cone decomposition data for

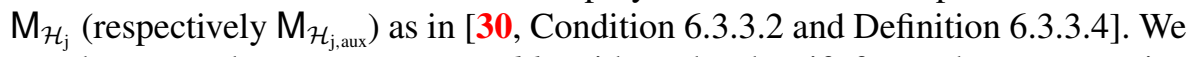
say that $\Sigma_{\mathrm{j}}$ and $\Sigma_{\mathrm{j}, \text { aux }}$ are compatible with each other if, for each representative $\left(Z_{\mathcal{H}_{\mathrm{j}}}, \Phi_{\mathcal{H}_{\mathrm{j}}}, \delta_{\mathcal{H}_{\mathrm{j}}}\right)$ of cusp label of $\mathrm{M}_{\mathcal{H}_{\mathrm{j}}}$ with assigned representative $\left(\mathrm{Z}_{\mathcal{H}_{\mathrm{j}, \text { aux }}}, \Phi_{\mathcal{H}_{\mathrm{j}, \text { aux }}}\right.$, $\delta_{\mathcal{H}_{\mathrm{j}, \text { aux }}}$ ) of cusp label of $\mathrm{M}_{\mathcal{H}_{\mathrm{j} \text {,aux }}}$ as in (5.8), the image of each $\sigma_{\mathrm{j}} \in \Sigma_{\Phi_{\mathcal{H}_{\mathrm{j}}}}$ under the embedding (5.10) is contained in some cone $\sigma_{\mathrm{j}, \text { aux }} \in \Sigma_{\Phi_{\mathcal{H}_{\mathrm{j}, \mathrm{aux}}}}$ In this case, we say that $\left(\Phi_{\mathcal{H}_{\mathrm{j}, \text { aux }}}, \delta_{\mathcal{H}_{\mathrm{j}, \text { aux }}}, \sigma_{\mathrm{j} \text {,aux }}\right)$ is assigned to $\left(\Phi_{\mathcal{H}_{\mathrm{j}}}, \delta_{\mathcal{H}_{\mathrm{j}}}, \sigma_{\mathrm{j}}\right)$, and (since this is compatible with the equivalence relations) we also say that $\left[\left(\Phi_{\mathcal{H}_{\mathrm{j} \text {,aux }}}, \delta_{\mathcal{H}_{\mathrm{j} \text {,aux }}}, \sigma_{\mathrm{j} \text {,aux }}\right)\right]$ is assigned to $\left[\left(\Phi_{\mathcal{H}_{\mathrm{j}}}, \delta_{\mathcal{H}_{\mathrm{j}}}, \sigma_{\mathrm{j}}\right)\right]$. Suppose $\Sigma_{\mathrm{j}}$ is induced by $\Sigma$ as in Lemma 3.21, and that $\sigma_{\mathrm{j}} \in \Sigma_{\Phi_{\mathcal{H}_{\mathrm{j}}}}$ is the image of some $\sigma \in \Sigma_{\Phi_{\mathcal{H}}}$ under (3.12). Then we also say that $\left(\Phi_{\mathcal{H}_{\mathrm{j} \text {,aux }}}, \delta_{\mathcal{H}_{\mathrm{j} \text {,aux }}}, \sigma_{\mathrm{j} \text {,aux }}\right)$ is assigned to $\left(\Phi_{\mathcal{H}}, \delta_{\mathcal{H}}, \sigma\right)$, and that $\left[\left(\Phi_{\mathcal{H}_{\mathrm{j}, \text { aux }}}, \delta_{\mathcal{H}_{\mathrm{j}, \text { aux }}}, \sigma_{\mathrm{j} \text {,aux }}\right)\right]$ is assigned to $\left[\left(\Phi_{\mathcal{H}}, \delta_{\mathcal{H}}, \sigma\right)\right]$.

Proposition 5.24. With assumptions as in Proposition 4.12, for each $\mathrm{j} \in \mathrm{J}$, there exists compatible choices $\Sigma_{\mathrm{j}}$ (respectively $\Sigma_{\mathrm{j}, \text { aux }}$ ) of admissible smooth rational polyhedral cone decomposition data for $\mathrm{M}_{\mathcal{H}_{\mathrm{j}}}$ (respectively $\mathrm{M}_{\mathcal{H}_{\mathrm{j}, \text { aux }}}$ ) such that $\Sigma_{\mathrm{j}}$ and $\Sigma_{\mathrm{j} \text {,aux }}$ are compatible with each other as in Definition 5.23, and such 
that the morphism (4.13) canonically extends to a morphism

$$
\mathrm{M}_{\mathcal{H}, \Sigma}^{\text {tor }} \rightarrow \mathrm{M}_{\mathcal{H}_{\mathrm{j}, \text { aux }}, \Sigma_{\mathrm{j}, \text { aux }}}^{\text {tor }} \underset{\mathbb{Z}}{\otimes} \mathbb{Q}
$$

which is the composition of (3.22) with a morphism

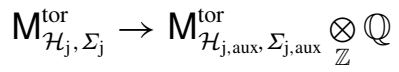

extending (4.14), where $\mathrm{M}_{\mathcal{H}, \Sigma}^{\text {tor }}, \mathrm{M}_{\mathcal{H}_{\mathrm{j}}, \Sigma_{\mathrm{j}}}^{\text {tor }}$, and $\mathrm{M}_{\mathcal{H}_{\mathrm{j}, \text { aux }, \Sigma_{\mathrm{j}, \text { aux }}}^{\text {tor }}}$ are toroidal compactifications of $\mathrm{M}_{\mathcal{H}}, \mathrm{M}_{\mathcal{H}_{\mathrm{j}}}$, and $\mathrm{M}_{\mathcal{H}_{\mathrm{j}, \text { aux }}}$, respectively, as in [30, Theorem 6.4.1.1]. Under the morphism (5.25) (respectively (5.26)), the $\left[\left(\Phi_{\mathcal{H}}, \delta_{\mathcal{H}}, \sigma\right)\right]$-stratum $\mathrm{Z}_{\left[\left(\Phi_{\mathcal{H}}, \delta_{\mathcal{H}}, \sigma\right)\right]}$ of $\mathrm{M}_{\mathcal{H}, \Sigma}^{\text {tor }}$ (respectively the $\left[\left(\Phi_{\mathcal{H}_{\mathrm{j}}}, \delta_{\mathcal{H}_{\mathrm{j}}}, \sigma_{\mathrm{j}}\right)\right]$-stratum $\mathrm{Z}_{\left[\left(\Phi_{\mathcal{H}_{\mathrm{j}}}, \delta_{\mathcal{H}_{\mathrm{j}}}, \sigma_{\mathrm{j}}\right)\right]}$ of

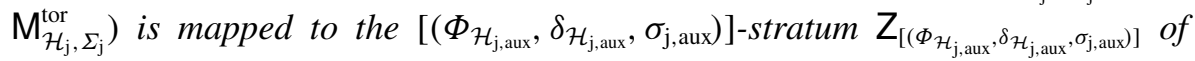
$\mathrm{M}_{\mathcal{H}_{\mathrm{j}, \text { aux }}, \Sigma_{\mathrm{j}, \text { aux }}}^{\text {to }}$ exactly when the equivalence class $\left[\left(\Phi_{\mathcal{H}_{\mathrm{j}, \text { aux }}}, \delta_{\mathcal{H}_{\mathrm{j}, \text { aux }}}, \sigma_{\mathrm{j}, \text { aux }}\right)\right]$ is assigned to the equivalence class $\left[\left(\Phi_{\mathcal{H}}, \delta_{\mathcal{H}}, \sigma\right)\right]$ (respectively $\left[\left(\Phi_{\mathcal{H}_{\mathrm{j}}}, \delta_{\mathcal{H}_{\mathrm{j}}}, \sigma_{\mathrm{j}}\right)\right]$ ) as in Definition 5.23.

Let $\left(G_{\mathrm{j}}, \lambda_{\mathrm{j}}, i_{\mathrm{j}}, \alpha_{\mathcal{H}_{\mathrm{j}}}\right)$ (respectively $\left.\left(G_{\mathrm{j}, \text { aux }}, \lambda_{\mathrm{j} \text {,aux }}, i_{\mathrm{j}, \text { aux }}, \alpha_{\mathcal{H}_{\mathrm{j}, \text { aux }}}\right)\right)$ denote the degenerating family of type $\mathrm{M}_{\mathcal{H}_{\mathrm{j}}}$ (respectively $\mathrm{M}_{\mathcal{H}_{\mathrm{j}, \text { aux }}}$ ) over $\mathrm{M}_{\mathcal{H}_{\mathrm{j}}, \Sigma_{\mathrm{j}}}^{\text {tor }}$ (respectively $\left.\mathrm{M}_{\mathcal{H}_{\mathrm{j}, \text { aux }}, \Sigma_{\mathrm{j}, \text { aux }}}^{\text {tor }}\right)$ as in [30, Theorem 6.4.1.1]. Then the pullback of $G_{\mathrm{j}, \text { aux }}$ to $\mathrm{M}_{\mathcal{H}_{\mathrm{j}}, \Sigma_{\mathrm{j}}}^{\text {tor }}$ (under (5.26)) is isomorphic to $G_{\mathrm{j}}^{\times a_{\mathrm{j}, 1}} \times_{\mathrm{M}_{\mathcal{H}_{\mathrm{j}}, \Sigma_{\mathrm{j}}}^{\text {tor }}}\left(G_{\mathrm{j}}^{\vee}\right)^{\times a_{\mathrm{j}, 2}}$, and the pullback of $\left(G_{\mathrm{j}, \text { aux }}\right.$, $\left.\lambda_{\mathrm{j}, \text { aux }}, i_{\mathrm{j}, \text { aux }}, \alpha_{\mathcal{H}_{\mathrm{j}, \text { aux }}}\right)$ to $\mathrm{M}_{\mathcal{H}_{\mathrm{j}}, \Sigma_{\mathrm{j}}}^{\text {tor }}$ satisfies analogs of the characterizing properties in Proposition 4.12 (in fact, by [52, IX, 1.4], [10, Ch. I, Proposition 2.7], or [30, Proposition 3.3.1.5], the last pullback is determined up to unique isomorphism by its restriction to $\mathrm{M}_{\mathcal{H}_{\mathrm{j}}}$, which is then characterized by the properties stated in Proposition 4.12).

The morphisms (5.25), for all $\mathrm{j} \in \mathrm{J}$, induce a morphism

$$
\mathrm{M}_{\mathcal{H}, \Sigma}^{\text {tor }} \rightarrow \prod_{\mathrm{j} \in \mathrm{J}} \mathrm{M}_{\mathcal{H}_{\mathrm{j}, \text { aux }}, \Sigma_{\mathrm{j}, \text { aux }}}^{\text {tor }} \underset{\mathbb{Z}}{\otimes} \mathbb{Q}
$$

extending (4.15).

Proof. Let us fix the choice of $\mathrm{j} \in \mathrm{J}$. As in (2) of Lemma 4.1 and as in the proof of Proposition 4.12, consider $G_{\mathrm{j}, \text { aux }}^{\Delta}:=G_{\mathrm{j}}^{\times\left(a_{\mathrm{j}, 1}+a_{\mathrm{j}, 2}\right)}, G_{\mathrm{j}, \text { aux }}^{\Delta, \vee}:=\left(G_{\mathrm{j}}^{\vee}\right)^{\times\left(a_{\mathrm{j}, 1}+a_{\mathrm{j}, 2}\right)}$, $G_{\mathrm{j}, \text { aux }}^{\nabla}:=G_{\mathrm{j}}^{\times a_{\mathrm{j}, 1}} \times_{\mathrm{M}_{\mathcal{H}_{\mathrm{j},}, \Sigma_{\mathrm{j}}}^{\text {tor }}}\left(G_{\mathrm{j}}^{\vee}\right)^{\times a_{\mathrm{j}, 2},}$, and $G_{\mathrm{j}, \text { aux }}^{\nabla, \vee}:=\left(G_{\mathrm{j}}^{\vee}\right)^{\times a_{\mathrm{j}, 1}} \times_{\mathrm{M}_{\mathcal{H}_{\mathrm{j}}, \Sigma_{\mathrm{j}}}^{\text {tor }}} G_{\mathrm{j}}^{\times a_{\mathrm{j}, 2}}$, which are fiber products over $\mathrm{M}_{\mathcal{H}_{\mathrm{j}}, \Sigma_{\mathrm{j}}}^{\text {tor }}$, whose pullbacks to $\mathrm{M}_{\mathcal{H}}$ can be canonically identified with $A_{\mathrm{j}, \text { aux }}^{\Delta}, A_{\mathrm{j}, \text { aux }}^{\Delta, \vee}, A_{\mathrm{j}, \text { aux }}^{\nabla}$, and $A_{\mathrm{j}, \text { aux }}^{\nabla, \vee}$, respectively. Consider

$$
f:=\operatorname{Id}_{G_{\mathrm{j}}}^{a_{\mathrm{j}, 1}} \underset{\mathrm{M}_{\mathcal{H}_{\mathrm{j}}, \Sigma_{\mathrm{j}}}^{\mathrm{or}}}{\times} \lambda_{\mathrm{j}}^{a_{\mathrm{j}, 2}}: G_{\mathrm{j}, \text { aux }}^{\Delta} \rightarrow G_{\mathrm{j}, \text { aux }}^{\nabla}
$$


and

$$
f^{\vee}:=\operatorname{Id}_{G_{\mathrm{j}}^{\vee}}^{a_{\mathrm{j}, 1}} \underset{\mathrm{M}_{\mathcal{H}}, \Sigma_{\mathrm{j}}}{\times} \underset{\mathrm{tor}}{\times} \lambda_{\mathrm{j}, 2}^{a_{\mathrm{j}, 2}}: G_{\mathrm{j}, \text { aux }}^{\nabla, \vee} \rightarrow G_{\mathrm{j}, \text { aux }}^{\Delta, \vee}
$$

whose pullbacks to $M_{\mathcal{H}_{\mathrm{j}}}$ are dual isogenies of each other. Let $\lambda_{\mathrm{j} \text {,aux }}^{\Delta}$ be defined by $\lambda_{\mathrm{j}}$ and the morphism $(\cdot, \cdot)_{\mathrm{j}}^{*}$ as in Lemma 4.1, and let

$$
i_{\mathrm{j}, \text { aux }}^{\Delta}: \mathcal{O}_{\text {aux }} \rightarrow \operatorname{End}_{\mathrm{M}_{\mathcal{H}}^{\text {tor }}, \Sigma_{\mathrm{j}}}\left(G_{\mathrm{j}, \text { aux }}^{\Delta}\right)
$$

be induced by the restriction of $i_{\mathrm{j}}$ to $\mathcal{O}_{\text {aux }}$. By (2) of Lemma 4.1, and by [52, IX, 1.4], [10, Ch. I, Proposition 2.7], or [30, Proposition 3.3.1.5],

$$
\lambda_{\mathrm{j}, \text { aux }}^{\nabla}:=\left(f^{\vee}\right)^{-1} \circ \lambda_{\mathrm{j} \text {,aux }}^{\Delta} \circ f: G_{\mathrm{j}, \text { aux }}^{\nabla} \rightarrow G_{\mathrm{j}, \text { aux }}^{\nabla, \vee}
$$

is an isogeny (not just a $\mathbb{Q}^{\times}$-isogeny) of degree prime to $p$ whose pullback to $\mathrm{M}_{\mathcal{H}_{\mathrm{j}}}$ is a polarization, and we have an

$$
i_{\mathrm{j}, \text { aux }}^{\nabla}: \mathcal{O}_{\text {aux }} \rightarrow \operatorname{End}_{\mathrm{M}_{\mathcal{H}}^{\text {tor }}, \Sigma_{\mathrm{j}}}\left(G_{\mathrm{j}, \text { aux }}^{\nabla}\right)
$$

uniquely extending its pullback to $M_{\mathcal{H}_{j}}$. Together with the $\alpha_{\mathcal{H}_{j} \text {,aux }}^{\nabla}$ over $M_{\mathcal{H}_{j}}$ constructed in the proof of Proposition 4.12, we obtain a degenerating family $\left(G_{\mathrm{j}, \text { aux }}^{\nabla}, \lambda_{\mathrm{j}, \text { aux }}^{\nabla}, i_{\mathrm{j}, \text { aux }}^{\nabla}, \alpha_{\mathcal{H}_{\mathrm{j}, \text { aux }}}^{\nabla}\right)$ of type $\mathrm{M}_{\mathcal{H}_{\mathrm{j}, \text { aux }}}$ over $\mathrm{M}_{\mathcal{H}_{\mathrm{j}}, \Sigma_{\mathrm{j}}}^{\text {tor }}$.

To show that $\left(G_{\mathrm{j} \text {,aux }}^{\nabla}, \lambda_{\mathrm{j}, \text { aux }}^{\nabla}, i_{\mathrm{j} \text {,aux }}^{\nabla}, \alpha_{\mathcal{H}_{\mathrm{j}, \text { aux }}}^{\nabla}\right) \rightarrow \mathrm{M}_{\mathcal{H}_{\mathrm{j}}, \Sigma_{\mathrm{j}}}^{\text {tor }}$ is canonically isomorphic to the pullback of $\left(G_{\mathrm{j}, \text { aux }}, \lambda_{\mathrm{j}, \text { aux }}, i_{\mathrm{j} \text {,aux }}, \alpha_{\mathcal{H}_{\mathrm{j}, \text { aux }}}\right) \rightarrow \mathrm{M}_{\mathcal{H}_{\mathrm{j}, \text { aux }}, \Sigma_{\mathrm{j}, \text { aux }}}^{\text {tor }}$ under a canonically determined morphism (5.26), we need to verify the condition as in [30, Theorem 6.4.1.1(6)].

In the association of degeneration data, over any $\operatorname{Spec}(V) \rightarrow \mathrm{M}_{\mathcal{H}_{\mathrm{j}}, \Sigma_{\mathrm{j}}}^{\text {tor }}$ such that $V$ is a complete discrete valuation ring with algebraically closed residue field $k$ and valuation $v: \operatorname{Inv}(V) \rightarrow \mathbb{Z}$, and such that $\operatorname{Spec}(\operatorname{Frac}(V))$ is mapped to a point $s$ of $\mathrm{M}_{\mathcal{H}_{\mathrm{j}}}$, and for any lifting $\hat{\alpha}_{\bar{s}}: L_{\mathrm{j}} \otimes_{\mathbb{Z}} \hat{\mathbb{Z}} \stackrel{\sim}{\rightarrow} \mathrm{T} G_{\mathrm{j}, \bar{s}}$ at a geometric point $\bar{s}$ above $s$, the (noncanonical) filtration $\mathrm{Z}_{\mathrm{j}}$ is defined to be the pullback of the geometric filtration $0 \subset \mathrm{T} T_{\mathrm{j}, \bar{s}} \subset \mathrm{T} G_{\mathrm{j}, \bar{s}}^{\natural} \subset \mathrm{T} G_{\mathrm{j}, \bar{s}}$, whose $\mathcal{H}_{\mathrm{j}}$-orbit $\mathrm{Z}_{\mathcal{H}_{\mathrm{j}}}$ is uniquely determined by $\alpha_{\mathcal{H}_{\mathrm{j}}}$. If we define $\hat{\alpha}_{\bar{s}}^{\nabla, p}: L_{\mathrm{j}, \text { aux }} \otimes_{\mathbb{Z}} \hat{\mathbb{Z}}^{p} \stackrel{\sim}{\rightarrow} \mathrm{T}^{p} G_{\mathrm{j}, \text { aux }, \bar{s}}^{\nabla}$ by $\hat{\alpha}_{\bar{s}}$ as in the proof of Proposition 4.12 , then the filtration $Z_{\mathrm{j}, \text { aux }}$ defined by $\mathrm{Z}_{\mathrm{j}}$ as in (5.1) agrees with the pullback of the geometric filtration $0 \subset \mathrm{T}^{p} T_{\mathrm{j}, \text { aux }, \bar{s}}^{\nabla} \subset \mathrm{T}^{p} G_{\mathrm{j}, \text { aux }, \bar{s}}^{\nabla, \natural} \subset \mathrm{T}^{p} G_{\mathrm{j}, \text { aux }, \bar{s}}^{\nabla}$, because this last geometric filtration on $\mathrm{T}^{p} G_{\mathrm{j}, \text { aux }, \bar{s}}^{\nabla}$ is induced by the geometric filtration $0 \subset \mathrm{V}^{p} T_{\mathrm{j}, \text { aux }, \bar{s}}^{\nabla} \subset \mathrm{V}^{p} G_{\mathrm{j}, \text { aux }, \bar{s}}^{\nabla, \natural} \subset \mathrm{V}^{p} G_{\mathrm{j}, \text { aux }, \bar{s}}^{\nabla}$ on $\mathrm{V}^{p} G_{\mathrm{j}, \text { aux }, \bar{s}}^{\nabla}$, whose pullback under the isomorphism $\mathrm{V}(f): \mathrm{V}^{p} G_{\mathrm{j}, \text { aux }, \bar{s}}^{\Delta} \stackrel{\sim}{\rightarrow} \mathrm{V}^{p} G_{\mathrm{j}, \text { aux }, \bar{s}}^{\nabla}$ agrees with the geometric filtration $0 \subset \mathrm{V}^{p} T_{\mathrm{j}, \text { aux }, \bar{s}}^{\Delta} \subset \mathrm{V}^{p} G_{\mathrm{j}, \text { aux }, \bar{s}}^{\Delta, \natural} \subset \mathrm{V}^{p} G_{\mathrm{j}, \text { aux }, \bar{s}}^{\Delta}$ on $\mathrm{V}^{p} G_{\mathrm{j}, \text { aux }, \bar{s}}^{\Delta}$ (which naturally agrees with the geometric filtration induced by $0 \subset \mathrm{V} T_{\mathrm{j}, \bar{s}} \subset \mathrm{V} G_{\mathrm{j}, \bar{s}}^{\natural} \subset \mathrm{V} G_{\mathrm{j}, \bar{s}}$ on $\left.\mathrm{V} G_{\mathrm{j}, \bar{s}}\right)$. 
Suppose, under the equivalence of categories in [30, Theorem 5.3.1.19],

$$
\left(B_{\mathrm{j}}, \lambda_{B_{\mathrm{j}}}, i_{B_{\mathrm{j}}}, X_{\mathrm{j}}, Y_{\mathrm{j}}, \phi_{\mathrm{j}}, c_{\mathrm{j}}, c_{\mathrm{j}}^{\vee}, \tau_{\mathrm{j}},\left[\alpha_{\mathcal{H}_{\mathrm{j}}}^{\natural}\right]\right)
$$

is the object in $\operatorname{DD}_{\mathrm{PEL}, \mathrm{M}_{\mathcal{H}_{\mathrm{j}}}}(V)$ associated with the object in $\operatorname{DEG}_{\mathrm{PEL}, \mathrm{M}_{\mathcal{H}_{\mathrm{j}}}}(V)$ defined by the pullback of the degenerating family $\left(G_{\mathrm{j}}, \lambda_{\mathrm{j}}, i_{\mathrm{j}}, \alpha_{\mathcal{H}_{\mathrm{j}}}\right)$ over $\mathrm{M}_{\mathcal{H}_{\mathrm{j}}, \Sigma_{\mathrm{j}}}^{\text {tor }}$ under some morphism $\operatorname{Spec}(V) \rightarrow \mathrm{M}_{\mathcal{H}_{\mathrm{j}}, \Sigma_{\mathrm{j}}}^{\text {tor }}$ as above, and suppose

$$
\left(B_{\mathrm{j}, \text { aux }}, \lambda_{B_{\mathrm{j}, \text { aux }}}, i_{B_{\mathrm{j}, \text { aux }}}, X_{\mathrm{j}, \text { aux }}, Y_{\mathrm{j}, \text { aux }}, c_{\mathrm{j}, \text { aux }}, c_{\mathrm{j} \text {,aux }}^{\vee}, \tau_{\mathrm{j}, \text { aux }},\left[\alpha_{\mathcal{H}_{\mathrm{j}, \text { aux }}}^{\natural}\right]\right)
$$

is the object in $\operatorname{DD}_{\mathrm{PEL}, \mathrm{M}_{\mathcal{H}_{\mathrm{j}} \text {,aux }}}(V)$ associated with the object in $\operatorname{DEG}_{\mathrm{PEL}, \mathrm{M}_{\mathcal{H}_{\mathrm{j}, \text { aux }}}}(V)$ defined by the pullback of the degenerating family $\left(G_{\mathrm{j}, \text { aux }}^{\nabla}, \lambda_{\mathrm{j}, \text { aux }}^{\nabla}, i_{\mathrm{j}, \text { aux }}^{\nabla}, \alpha_{\mathcal{H}_{\mathrm{j}, \text { aux }}}^{\nabla}\right)$ over $\mathrm{M}_{\mathcal{H}_{\mathrm{j}}, \Sigma_{\mathrm{j}}}^{\text {tor }}$ under the same $\operatorname{Spec}(V) \rightarrow \mathrm{M}_{\mathcal{H}_{\mathrm{j}}, \Sigma_{\mathrm{j}}}^{\text {tor }}$. Then (5.29) is induced by (5.28) in a precise sense (whose details we omit), and we have the following. Under the assignment (5.7), the cusp label $\left[\left(\mathrm{Z}_{\mathcal{H}_{\mathrm{j}}}, \Phi_{\mathcal{H}_{\mathrm{j}}}=\left(X_{\mathrm{j}}, Y_{\mathrm{j}}, \phi_{\mathrm{j}}, \varphi_{-2, \mathcal{H}_{\mathrm{j}}}, \varphi_{0, \mathcal{H}_{\mathrm{j}}}\right), \delta_{\mathcal{H}_{\mathrm{j}}}\right)\right]$ determined by (5.28) gives the cusp label $\left[\left(Z_{\mathcal{H}_{\mathrm{j}, \text { aux }}}, \Phi_{\mathcal{H}_{\mathrm{j}, \text { aux }}}, \delta_{\mathcal{H}_{\mathrm{j}, \text { aux }}}\right)\right]$ determined by (5.29). Given any representative $\left(Z_{\mathcal{H}_{\mathrm{j}}}, \Phi_{\mathcal{H}_{\mathrm{j}}}, \delta_{\mathcal{H}_{\mathrm{j}}}\right)$ of $\left[\left(\mathrm{Z}_{\mathcal{H}_{\mathrm{j}}}, \Phi_{\mathcal{H}_{\mathrm{j}}}, \delta_{\mathcal{H}_{\mathrm{j}}}\right)\right]$, the assignment (5.8) gives a representative $\left(\mathrm{Z}_{\mathcal{H}_{\mathrm{j}, \text { aux }}}, \Phi_{\mathcal{H}_{\mathrm{j}, \text { aux }}}, \delta_{\mathcal{H}_{\mathrm{j}, \text { aux }}}\right)$ of $\left[\left(\mathrm{Z}_{\mathcal{H}_{\mathrm{j}, \text { aux }}}, \Phi_{\mathcal{H}_{\mathrm{j}, \text { aux }}}\right.\right.$, $\left.\left.\delta_{\mathcal{H}_{\mathrm{j} \text {,aux }}}\right)\right]$. With such choices of $\left(\mathrm{Z}_{\mathcal{H}_{\mathrm{j}}}, \Phi_{\mathcal{H}_{\mathrm{j}}}, \delta_{\mathcal{H}_{\mathrm{j}}}\right)$ and $\left(\mathrm{Z}_{\mathcal{H}_{\mathrm{j}, \text { aux }}}, \Phi_{\mathcal{H}_{\mathrm{j} \text {,aux }}}, \delta_{\mathcal{H}_{\mathrm{j}, \text { aux }}}\right)$, if

$$
B_{\mathrm{j}}: \mathbf{S}_{\Phi_{\mathcal{H}_{\mathrm{j}}}} \rightarrow \operatorname{Inv}(V)
$$

and

$$
B_{\mathrm{j}, \text { aux }}: \mathbf{S}_{\Phi_{\mathcal{H}_{\mathrm{j}, \text { aux }}}} \rightarrow \operatorname{Inv}(V)
$$

are determined by (5.28) and (5.29), respectively, then (5.9) maps

$$
v \circ B_{\mathrm{j}}: \mathbf{S}_{\Phi_{\mathcal{H}_{\mathrm{j}}}} \rightarrow \mathbb{Z} \hookrightarrow \mathbb{Q}
$$

to

$$
v \circ B_{\mathrm{j}, \text { aux }}: \mathbf{S}_{\Phi_{\mathcal{H}_{\mathrm{j}, \text { aux }}}} \rightarrow \mathbb{Z} \hookrightarrow \mathbb{Q}
$$

because $\lambda_{\mathrm{j} \text {,aux }}^{\Delta}$ is induced by $\lambda_{\mathrm{j}}$ and $(\cdot, \cdot)_{\mathrm{j}}$. Consequently, if $v \circ B_{\mathrm{j}}$ defines an element of $\sigma_{\mathrm{j}} \in \Sigma_{\Phi_{\mathcal{H}_{\mathrm{j}}}}$, and if the image of $\sigma_{\mathrm{j}}$ under (5.10) is contained in some $\sigma_{\mathrm{j}, \text { aux }} \in$ $\Sigma_{\Phi_{\mathcal{H}_{\mathrm{j}, \text { aux }}}}$, then $v \circ B_{\mathrm{j}, \text { aux }}$ defines an element of $\sigma_{\mathrm{j} \text {,aux }}$.

Thus, if $\Sigma_{\mathrm{j}}$ and $\Sigma_{\mathrm{j} \text {,aux }}$ are compatible with each other as in Definition 5.23, by considering all morphisms $\operatorname{Spec}(V) \rightarrow \mathrm{M}_{\mathcal{H}_{\mathrm{j}}, \Sigma_{\mathrm{j}}}^{\text {tor }}$ as above, we see that the degenerating family $\left(G_{\mathrm{j}, \text { aux }}^{\nabla}, \lambda_{\mathrm{j}, \text { aux }}^{\nabla}, i_{\mathrm{j}, \text { aux }}^{\nabla}, \alpha_{\mathcal{H}_{\mathrm{j}, \text { aux }}}^{\nabla}\right)$ satisfies the condition as in [30, Theorem 6.4.1.1(6)], as desired.

Consider the invertible sheaves

$$
\omega_{\mathrm{M}_{\mathcal{H}, \Sigma}^{\text {tor }}}:=\wedge^{\text {top }} \underline{\operatorname{Lie}}_{G / \mathrm{M}_{\mathcal{H}, \Sigma}^{\mathrm{tor}}}^{\vee}
$$


over $\mathrm{M}_{\mathcal{H}, \Sigma}^{\text {tor }}$ and, for each $\mathrm{j} \in \mathrm{J}$,

$$
\omega_{\mathrm{M}_{\mathcal{H}_{\mathrm{j}}, \Sigma_{\mathrm{j}}}^{\text {tor }}}:=\wedge^{\text {top }} \underline{\mathrm{Lie}}_{G_{\mathrm{j}} / \mathrm{M}_{\mathcal{H}_{\mathrm{j}}, \Sigma_{\mathrm{j}}}^{\mathrm{tor}}}^{\vee}
$$

$\operatorname{over} \mathrm{M}_{\mathcal{H}_{\mathrm{j}}, \Sigma_{\mathrm{j}}}^{\text {tor }}$ and

$$
\omega_{\mathrm{M}_{\mathcal{H}_{\mathrm{j}, \text { aux }}, \Sigma_{\mathrm{j}, \text { aux }}}^{\text {tor }}}:=\wedge^{\text {top }} \underline{\mathrm{Lie}}_{G_{\mathrm{j}, \text { aux }} / \mathrm{M}_{\mathcal{H}_{\mathrm{j}, \text { aux }}, \Sigma_{\mathrm{j}, \text { aux }}}^{\mathrm{tor}}}^{\vee}
$$

over $\mathrm{M}_{\mathcal{H}_{\mathrm{j}, \text { aux }}, \Sigma_{\mathrm{j}, \text { aux }}}^{\text {tor }}$. We shall denote the pullback of $\omega_{\mathrm{M}_{\mathcal{H}, \Sigma}^{\text {tor }}}$ (respectively $\omega_{\mathrm{M}_{\mathcal{H}}, \Sigma_{\mathrm{j}}}^{\text {tor }}$, respectively $\omega_{\mathrm{M}_{\mathcal{H}_{\mathrm{j}, \text { aux }}}^{\text {tor }}}$ ) to $\mathrm{M}_{\mathcal{H}}$ (respectively $\mathrm{M}_{\mathcal{H}_{\mathrm{j}}}$, respectively $\mathrm{M}_{\mathcal{H}_{\mathrm{j} \text {,aux }}}$ ) by $\omega_{\mathrm{M}_{\mathcal{H}}}$ (respectively $\omega_{\mathrm{M}_{\mathcal{H}_{\mathrm{j}}}}$, respectively $\omega_{\mathrm{M}_{\mathcal{H}_{\mathrm{j}, \text { aux }}}}$ ), which is independent of the choice of $\Sigma$ (respectively $\Sigma_{\mathrm{j}}$, respectively $\Sigma_{\mathrm{j} \text {,aux }}$ ).

LEMmA 5.30. Suppose $\mathrm{j} \in \mathrm{J}$. The pullback of $\omega_{\mathrm{M}_{\mathcal{H}_{\mathrm{j}}, \Sigma_{\mathrm{j}}}^{\text {tor }}}$ under the canonical isomorphism (3.22) is isomorphic to $\omega_{\mathrm{M}_{\mathcal{H}, \Sigma}^{\text {tor }}}$. There exists an integer $1 \leqslant a_{\mathrm{j}, 0} \leqslant 2$ such that the pullback of $\omega_{\mathrm{M}_{\mathcal{H}, \text { aux }}^{\text {tor }}, \Sigma_{\mathrm{j}, \text { aux }}}^{\otimes a_{\mathrm{j},}}$ to $\mathrm{M}_{\mathcal{H}, \Sigma}^{\text {tor }}$ (respectively $\mathrm{M}_{\mathcal{H}_{\mathrm{j}}, \Sigma_{\mathrm{j}}}^{\text {tor }}$ ) under the morphism (5.25) (respectively (5.26)) is isomorphic to $\omega_{\mathrm{M}_{\mathcal{H}, \Sigma}^{\mathrm{tor}}}^{\otimes a_{\mathrm{j}}}$ (respectively $\left.\omega_{\mathrm{M}_{\mathcal{H}_{\mathrm{j}}, \Sigma_{\mathrm{j}}}^{\mathrm{tor}}}^{\otimes a_{\mathrm{j}}}\right)$, where

$$
a_{\mathrm{j}}:=a_{\mathrm{j}, 0}\left(a_{\mathrm{j}, 1}+a_{\mathrm{j}, 2}\right)
$$

We may take $a_{\mathrm{j}, 0}=1$ when $a_{\mathrm{j}, 2}$ is even.

We shall henceforth fix a choice of $a_{\mathrm{j}, 0}$ (for each $\mathrm{j} \in \mathrm{J}$ ).

Proof of Lemma 5.30. The first assertion is because the pullback of $G_{\mathrm{j}}$ under (3.22) is $\mathbb{Q}^{\times}$-isogenous to $G$. As for the second assertion, consider also the invertible sheaf

$$
\omega_{\mathrm{M}_{\mathcal{H}_{\mathrm{j}}, \Sigma_{\mathrm{j}}}^{\prime \text { tor }}}:=\wedge^{\text {top }} \underline{\mathrm{Lie}}_{G_{\mathrm{j}}^{\vee} / \mathrm{M}_{\mathcal{H}_{\mathrm{j}}, \Sigma_{\mathrm{j}}}^{\mathrm{tor}}} .
$$

By Proposition 5.24, the pullback of $\omega_{\mathrm{M}_{\mathcal{H}, \text { aux }}^{\text {tor } \Sigma_{\mathrm{j}, \text { aux }}}}$ to $\mathrm{M}_{\mathcal{H}_{\mathrm{j}}, \Sigma_{\mathrm{j}}}^{\text {tor }}$ is canonically isomorphic to

$$
\omega_{\mathrm{M}_{\mathcal{H}_{\mathrm{j}}, \Sigma_{\mathrm{j}}}^{\text {tor }}}^{\otimes a_{\mathrm{j}}} \otimes_{\mathrm{M}_{\mathcal{H}_{\mathrm{j}}, \Sigma_{\mathrm{j}}}^{\text {tor }}}\left(\omega_{\mathrm{M}_{\mathcal{H}_{\mathrm{j}}, \Sigma_{\mathrm{j}}}^{\text {tor }}}^{\prime}\right)^{\otimes a_{\mathrm{j}, 2}} .
$$

By [39, IX, 2.4, and its proof], there exists an integer $1 \leqslant a_{\mathrm{j}, 0} \leqslant 2$ such that

$$
\omega_{\mathrm{M}_{\mathcal{H}_{\mathrm{j}}, \Sigma_{\mathrm{j}}}^{\mathrm{tor}, 0}}^{\otimes a_{\mathrm{j}}} \cong\left(\omega_{\mathrm{M}_{\mathcal{H}_{\mathrm{j}}, \Sigma_{\mathrm{j}}}^{\mathrm{tor}}}^{\prime}\right)^{\otimes a_{\mathrm{j}, 0}} .
$$

Hence, up to replacing $a_{\mathrm{j}, 0}$ with 1 when $a_{\mathrm{j}, 2}$ is even, the lemma follows. 
For each $\mathrm{j} \in \mathrm{J}$, let $\mathbf{M}_{\mathcal{H}_{\mathrm{j}, \text { aux }}}^{\text {min }}$ denote the minimal compactification of $\mathrm{M}_{\mathcal{H}_{\mathrm{j}, \text { aux }}}$ as in [30, Theorem 7.2.4.1], which is by construction a projective variety over $\operatorname{Spec}\left(\mathcal{O}_{F_{0, \mathrm{j}, \text { aux }},(p)}\right)$ containing $\mathrm{M}_{\mathcal{H}_{\mathrm{j} \text {,aux }}}$ as an open subscheme, under the assumption that $\mathcal{H}_{\mathrm{j} \text {,aux }}$ is neat. By [30, Theorem 7.2.4.1(3)], $\omega_{\mathrm{M}_{\mathcal{H}, \Sigma}^{\text {tor }}}$ and $\omega_{\mathrm{M}_{\mathcal{H}}, \Sigma_{\mathrm{j}}}^{\text {tor }}$ descend to ample invertible sheaves $\omega_{\mathrm{M}_{\mathcal{H}}^{\min }}$ and $\omega_{\mathrm{M}_{\mathcal{H}_{\mathrm{j}}}^{\min }}$ over $\mathrm{M}_{\mathcal{H}}^{\min }$ and $\mathrm{M}_{\mathcal{H}_{\mathrm{j}}}^{\min }$, respectively, and we have

$$
\mathrm{M}_{\mathcal{H}}^{\min } \cong \operatorname{Proj}\left(\bigoplus_{k \geqslant 0} \Gamma\left(\mathrm{M}_{\mathcal{H}, \Sigma}^{\text {tor }}, \omega_{\mathrm{M}_{\mathcal{H}, \Sigma}^{\text {tor }}}^{\otimes k}\right)\right)
$$

and

$$
\mathrm{M}_{\mathcal{H}_{\mathrm{j}}}^{\text {min }} \cong \operatorname{Proj}\left(\bigoplus_{k \geqslant 0} \Gamma\left(\mathrm{M}_{\mathcal{H}_{\mathrm{j}}, \Sigma_{\mathrm{j}}}^{\text {tor }}, \omega_{\mathrm{M}_{\mathcal{H}_{\mathrm{j}}, \Sigma_{\mathrm{j}}}^{\text {tor }}}^{\otimes k}\right)\right),
$$

which are compatible with the canonical isomorphisms (3.22) and (3.23), and with the canonical isomorphism in Lemma 5.30 between $\omega_{\mathrm{M}_{\mathcal{H}, \Sigma}^{\text {tor }}}$ and the pullback of $\omega_{\mathrm{M}_{\mathcal{H}_{\mathrm{j}}, \Sigma_{\mathrm{j}}}^{\text {tor }}}$ under (3.22). Similarly, $\omega_{\mathrm{M}_{\mathcal{H}_{\mathrm{j}, \text { aux }} \Sigma_{\mathrm{j}, \text { aux }}}^{\text {tor }}}$ descends to an ample invertible sheaf $\omega_{\mathrm{M}_{\mathcal{H}_{\mathrm{j} \text {,aux }}}^{\min }}$ over $\mathrm{M}_{\mathcal{H}_{\mathrm{j} \text {,aux }}}^{\min }$, and we have

$$
\mathrm{M}_{\mathcal{H}_{\mathrm{j}, \text { aux }}}^{\min } \cong \operatorname{Proj}\left(\bigoplus_{k \geqslant 0} \Gamma\left(\mathrm{M}_{\mathcal{H}_{\mathrm{j}, \text { aux }}, \Sigma_{\mathrm{j}, \text { aux }}}^{\text {tor }}, \omega_{\mathrm{M}_{\mathcal{H}_{\mathrm{j}, \text { aux }}, \Sigma_{\mathrm{j}, \text { aux }}}^{\otimes k}}^{\text {tor }}\right)\right)
$$

Proposition 5.34. With assumptions as in Proposition 4.12, for each $\mathrm{j} \in \mathrm{J}$, there exists a morphism

$$
\mathrm{M}_{\mathcal{H}}^{\min } \rightarrow \mathrm{M}_{\mathcal{H} \text {,aux }}^{\min } \underset{\mathbb{Z}}{\otimes} \mathbb{Q}
$$

extending (4.13) and compatible with (5.25), which is the composition of (3.23) with a morphism

$$
\mathrm{M}_{\mathcal{H}_{\mathrm{j}}}^{\min } \rightarrow \mathrm{M}_{\mathcal{H}_{\mathrm{j}, \text { aux }}}^{\min } \underset{\mathbb{Z}}{\otimes} \mathbb{Q}
$$

extending (4.14) and compatible with (5.26). Under the morphism (5.35) (respectively (5.36)), the $\left[\left(\Phi_{\mathcal{H}}, \delta_{\mathcal{H}}\right)\right]$-stratum $\mathrm{Z}_{\left[\left(\Phi_{\mathcal{H}}, \delta_{\mathcal{H}}\right)\right]}$ of $\mathrm{M}_{\mathcal{H}}^{\min }$ (respectively $\left[\left(\Phi_{\mathcal{H}_{\mathrm{j}}}, \delta_{\mathcal{H}_{\mathrm{j}}}\right)\right]$-stratum $\mathrm{Z}_{\left[\left(\Phi_{\mathcal{H}_{\mathrm{j}}}, \delta_{\mathcal{H}_{\mathrm{j}}}\right)\right]}$ of $\left.\mathrm{M}_{\mathcal{H}_{\mathrm{j}}}^{\mathrm{min}}\right)$ is mapped to the $\left[\left(\Phi_{\mathcal{H}_{\mathrm{j}, \text { aux }}}, \delta_{\mathcal{H}_{\mathrm{j}, \text { aux }}}\right)\right]$ stratum $\mathrm{Z}_{\left[\left(\Phi_{\mathcal{H}_{\mathrm{j}, \text { aux }}}, \delta_{\mathcal{H}_{\mathrm{j}} \text {,aux }}\right)\right]}$ of $\mathrm{M}_{\mathcal{H}_{\mathrm{j}, \text { aux }}}^{\text {min }}$ exactly when the cusp label $\left[\left(\Phi_{\mathcal{H}_{\mathrm{j}, \text { aux }}}, \delta_{\mathcal{H}_{\mathrm{j}, \text { aux }}}\right)\right]$ is assigned to the cusp label $\left[\left(\Phi_{\mathcal{H}}, \delta_{\mathcal{H}}\right)\right]$ (respectively $\left[\left(\Phi_{\mathcal{H}_{\mathrm{j}}}, \delta_{\mathcal{H}_{\mathrm{j}}}\right)\right]$ ) as in $(5.14)$ (respectively (5.7)) (with the filtrations $\mathrm{Z}_{\mathcal{H}}, \mathrm{Z}_{\mathcal{H}_{\mathrm{j}}}$, and $\mathrm{Z}_{\mathcal{H}_{\mathrm{j}, \text { aux }}}$ suppressed in the notation). If $a_{\mathrm{j}, 0} \geqslant 1$ and $a_{\mathrm{j}} \geqslant 1$ are integers as in Lemma 5.30, then the pullback of $\omega_{\mathrm{M}_{\mathcal{H}}^{\text {min,aux }}}^{\otimes a_{\mathrm{j}, 0}}$ to $\mathrm{M}_{\mathcal{H}}^{\min }$ (respectively $\mathrm{M}_{\mathcal{H}_{\mathrm{j}}}^{\min }$ ) is canonically isomorphic to $\omega_{\mathrm{M}_{\mathcal{H}}^{\min }}^{\otimes a_{\mathrm{j}}}$ (respectively $\omega_{\mathrm{M}_{\mathcal{H}_{j}}^{\text {min }}}^{\otimes a_{j}}$ ). 
The morphisms (5.35), for all $\mathrm{j} \in \mathrm{J}$, induce a morphism

$$
\mathrm{M}_{\mathcal{H}}^{\min } \rightarrow \prod_{\mathrm{j} \in \mathrm{J}} \mathrm{M}_{\mathcal{H}_{\mathrm{j}, \text { aux }}}^{\min } \otimes_{\mathbb{Z}} \mathbb{Q}
$$

extending (4.15) and compatible with (5.27).

Consequently, $\mathrm{M}_{\mathcal{H}}^{\min }$ is the normalization of $\prod_{\mathrm{j} \in \mathrm{J}} \mathrm{M}_{\mathcal{H}_{\mathrm{j}, \text { aux }}}^{\min } \otimes_{\mathbb{Z}} \mathbb{Q}$ in $\mathrm{M}_{\mathcal{H}}$ under the morphism $\mathrm{M}_{\mathcal{H}} \rightarrow \prod_{\mathrm{j} \in \mathrm{J}} \mathrm{M}_{\mathcal{H}_{\mathrm{j}, \text { aux }}}^{\text {min }} \otimes_{\mathbb{Z}} \mathbb{Q}$ induced by (4.13) and the canonical morphisms $\mathrm{M}_{\mathcal{H}_{\mathrm{j}, \text { aux }}} \otimes_{\mathbb{Z}} \mathbb{Q} \rightarrow \mathrm{M}_{\mathcal{H}_{\mathrm{j}, \text { aux }}}^{\min } \otimes_{\mathbb{Z}} \mathbb{Q}$ for all $\mathrm{j} \in \mathrm{J}$.

Proof. The first two paragraphs follow from Proposition 5.24, from Lemma 5.30, and from the universal properties of the projective spectra (5.31) and (5.33).

For each $\mathrm{j} \in \mathrm{J}$, since $\omega_{\mathrm{M}_{\mathcal{H}_{\mathrm{j}, \text { aux }}}^{\text {min }}}^{\otimes a_{\mathrm{j}}}$ is ample over $\mathrm{M}_{\mathcal{H}_{\mathrm{j} \text {,aux }}}^{\min }$, since $\omega_{\mathrm{M}_{\mathcal{H}}^{\min }}^{\otimes a_{\mathrm{j}}}$ is ample over $\mathrm{M}_{\mathcal{H}}^{\min }$, and since the pullback of the former is canonically isomorphic to the latter, the canonical morphism from $M_{\mathcal{H}}^{\min }$ to the normalization of $\prod_{j \in J} M_{\mathcal{H}_{\mathrm{j}, \text { aux }}}^{\min } \otimes_{\mathbb{Z}} \mathbb{Q}$ in $\mathrm{M}_{\mathcal{H}}$ is finite. Since both of them are normal, and since they share an open dense subscheme $\mathrm{M}_{\mathcal{H}}$, the third paragraph follows from Zariski's main theorem (see [14, III-1, 4.4.3, 4.4.11]), as desired.

\section{Minimal compactifications defined by normalization}

Proposition 6.1. Let $\overrightarrow{\mathrm{M}}_{\mathcal{H}}$ denote the normalization of $\prod_{\mathrm{j} \in \mathrm{J}} \mathrm{M}_{\mathcal{H}_{\mathrm{j}, \text { aux }}}$ in $\mathrm{M}_{\mathcal{H}}$ under the morphism

$$
\mathrm{M}_{\mathcal{H}} \rightarrow \prod_{\mathrm{j} \in \mathrm{J}} \mathrm{M}_{\mathcal{H}_{\mathrm{j}, \text { aux }}}
$$

induced by (4.15). Then $\overrightarrow{\mathrm{M}}_{\mathcal{H}}$ is a normal algebraic stack flat over

$$
\overrightarrow{\mathrm{S}}_{0}:=\operatorname{Spec}\left(\mathcal{O}_{F_{0},(p)}\right)
$$

equipped with a canonical isomorphism $\overrightarrow{\mathrm{M}}_{\mathcal{H}} \times_{\overrightarrow{\mathrm{S}}_{0}} \mathrm{~S}_{0} \cong \mathrm{M}_{\mathcal{H}}$ over $\mathrm{S}_{0}$, and with a canonical finite morphism

$$
\overrightarrow{\mathrm{M}}_{\mathcal{H}} \rightarrow \prod_{\mathrm{j} \in \mathrm{J}} \mathrm{M}_{\mathcal{H}_{\mathrm{j}, \text { aux }}}
$$

extending (4.15) and (6.2).

For each $\mathrm{j} \in \mathrm{J}$, the tautological tuple $\left(A_{\mathrm{j}}, \lambda_{\mathrm{j}}, i_{\mathrm{j}}, \alpha_{\mathcal{H}_{\mathrm{j}}}\right)$ over $\mathrm{M}_{\mathcal{H}_{\mathrm{j}}} \cong \mathrm{M}_{\mathcal{H}}$ (see (2.1)) extends to a degenerating family $\left(\vec{A}_{\mathrm{j}}, \vec{\lambda}_{\mathrm{j}}, \vec{i}_{\mathrm{j}}, \vec{\alpha}_{\mathcal{H}_{\mathrm{j}}}\right)$ of type $\mathrm{M}_{\mathcal{H}_{\mathrm{j}}}$ over $\overrightarrow{\mathrm{M}}_{\mathcal{H}}$ (see [30, Definition 5.3.2.1]), where $\left(\vec{A}_{\mathrm{j}}, \vec{\lambda}_{\mathrm{j}}\right)$ is a polarized abelian scheme with 


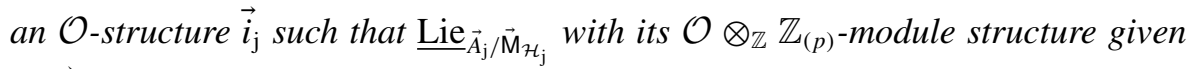
by $\vec{i}_{\mathrm{j}}$ satisfies the determinantal condition in [30, Definition 1.3.4.1] given by $\left(L_{\mathrm{j}} \otimes_{\mathbb{Z}} \mathbb{R},\langle\cdot, \cdot\rangle, h_{0}\right) \cong\left(L \otimes_{\mathbb{Z}} \mathbb{R},\langle\cdot, \cdot\rangle, h_{0}\right)$, and where $\vec{\alpha}_{\mathcal{H}_{\mathrm{j}}}$ is defined only over $\mathrm{M}_{\mathcal{H}_{\mathrm{j}}}$. If we denote by $\left(\vec{A}_{\mathrm{j}, \text { aux }}, \vec{\lambda}_{\mathrm{j}, \text { aux }}, \vec{i}_{\mathrm{j}, \text { aux }}, \vec{\alpha}_{\mathcal{H}_{\mathrm{j}, \text { aux }}}\right)$ the pullback of the tautological tuple $\left(A_{\mathrm{j}, \text { aux }}, \lambda_{\mathrm{j} \text {,aux }}, i_{\mathrm{j}, \text { aux }}, \alpha_{\mathcal{H}_{\mathrm{j}, \text { aux }}}\right)$ over $\mathrm{M}_{\mathcal{H}_{\mathrm{j} \text {,aux }}}$ under the morphism $\overrightarrow{\mathrm{M}}_{\mathcal{H}_{\mathrm{j}}} \rightarrow \mathrm{M}_{\mathcal{H}_{\mathrm{j}, \text { aux }}}$ induced by (6.3), then $\left(\vec{A}_{\mathrm{j}, \text { aux }}, \vec{\lambda}_{\mathrm{j}, \text { aux }}\right)$ is isomorphic to the polarized abelian scheme $\left(\vec{A}_{\mathrm{j}, \text { aux }}^{\nabla}, \vec{\lambda}_{\mathrm{j} \text {,aux }}^{\nabla}\right)$ defined by $\left(\vec{A}_{\mathrm{j}}, \vec{\lambda}_{\mathrm{j}}\right)$ as in (2) of Lemma $4.1, \vec{i}_{\mathrm{j}}$ is the unique extension of $i_{\mathrm{j}}$ over the noetherian normal base scheme $\overrightarrow{\mathrm{M}}_{\mathcal{H}}$ (by [52, IX, 1.4], [10, Ch. I, Proposition 2.7], or [30, Proposition 3.3.1.5]), and $\vec{\alpha}_{\mathcal{H}_{\mathrm{j}, \text { aux }}}$ is determined by $\alpha_{\mathcal{H}_{\mathrm{j}}}$ in the sense that its further pullback to $\mathrm{M}_{\mathcal{H}_{\mathrm{j}}} \cong \mathrm{M}_{\mathcal{H}}$ is determined by $\alpha_{\mathcal{H}_{\mathrm{j}}}$ as in Proposition 4.12. Then the invertible sheaf $\omega_{\mathrm{M}_{\mathcal{H}_{\mathrm{j}}}}$ over $\mathrm{M}_{\mathcal{H}_{\mathrm{j}}} \cong \mathrm{M}_{\mathcal{H}}$ extends to the invertible sheaf

$$
\omega_{\overrightarrow{\mathrm{M}}_{\mathcal{H}}, \mathrm{j}}:=\wedge^{\text {top }} \underline{\mathrm{Lie}}_{\overrightarrow{\mathrm{A}}_{\mathrm{j}} / \overrightarrow{\mathrm{M}}_{\mathcal{H}}}^{\vee}
$$

over $\overrightarrow{\mathrm{M}}_{\mathcal{H}}$. For each $\mathrm{j} \in \mathrm{J}$, let $a_{\mathrm{j}, 0} \geqslant 1$ and $a_{\mathrm{j}} \geqslant 1$ be integers as in Lemma 5.30, and let $a_{\mathrm{J}}:=\sum_{\mathrm{j} \in \mathrm{J}} a_{\mathrm{j}}$. Then the invertible sheaf $\omega_{\mathrm{M}_{\mathcal{H}}}^{\otimes a_{\mathrm{J}}}$ over $\mathrm{M}_{\mathcal{H}}$ extends to the ample invertible sheaf

$$
\omega_{\overrightarrow{\mathrm{M}}_{\mathcal{H}}, \mathrm{J}}:=\bigotimes_{\mathrm{j} \in \mathrm{J}} \omega_{\overrightarrow{\mathrm{M}}_{\mathcal{H}}, \mathrm{j}}^{\otimes a_{\mathrm{j}}}
$$

over $\overrightarrow{\mathrm{M}}_{\mathcal{H}}$, where each $\omega_{\overrightarrow{\mathrm{M}}_{\mathcal{H}}, \mathrm{j}}^{\otimes a_{\mathrm{j}}}$ is canonically isomorphic to the pullback of $\omega_{\mathrm{M}_{\mathcal{H}, \mathrm{j}, \text { aux }}}^{\otimes a_{\mathrm{j}}}$ under the morphism $\overrightarrow{\mathrm{M}}_{\mathcal{H}} \rightarrow \mathrm{M}_{\mathcal{H}_{\mathrm{j}, \text { aux }}}$ induced by (6.3), and where $\omega_{\overrightarrow{\mathrm{M}}_{\mathcal{H}}, \mathrm{J}}$ is canonically isomorphic to the pullback of $\bigotimes_{\mathrm{j} \in \mathrm{J}} \omega_{\mathrm{M}_{\mathcal{H}}, \mathrm{aux}}^{\otimes a_{\mathrm{j}, 0}}$ under (6.3).

We obtain the same normalization $\overrightarrow{\mathrm{M}}_{\mathcal{H}}$ (up to canonical isomorphism) satisfying the analogous properties if, for each $\mathrm{j} \in \mathrm{J}$, we replace $\mathcal{H}_{\mathrm{j}, \text { aux }}$ with any neat open compact subgroup of $\mathrm{G}_{\mathrm{j}, \text { aux }}\left(\hat{\mathbb{Z}}^{p}\right)$ still containing the image of $\mathcal{H}_{\mathrm{j}}$ under the homomorphism $\mathrm{G}_{\mathrm{j}}(\hat{\mathbb{Z}}) \rightarrow \mathrm{G}_{\mathrm{j}, \text { aux }}\left(\hat{\mathbb{Z}}^{p}\right)$ given by (4.10).

Up to canonical isomorphism, $\overrightarrow{\mathrm{M}}_{\mathcal{H}}$ depends only on the choices of linear algebraic data in Section 2, but not on the auxiliary choices in Sections 4 and 5.

Proof. The first paragraph is self-explanatory. As for the second paragraph, except for the ampleness of $\omega_{\overrightarrow{\mathrm{M}}_{\mathcal{H}}, \mathrm{J}}$, it suffices to show that, for each $\mathrm{j} \in \mathrm{J}$, the tautological $\left(A_{\mathrm{j}}, \lambda_{\mathrm{j}}\right)$ over $\mathrm{M}_{\mathcal{H}_{\mathrm{j}}} \cong \mathrm{M}_{\mathcal{H}}$ extends to some polarized abelian scheme $\left(\vec{A}_{\mathrm{j}}, \vec{\lambda}_{\mathrm{j}}\right)$ over $\overrightarrow{\mathrm{M}}_{\mathcal{H}}$ (once this is shown, the remainder of the paragraph will follow from the uniqueness of extensions by [52, IX, 1.4], [10, Ch. I, Proposition 2.7], or [30, Proposition 3.3.1.5]). Since the genus of $A_{\mathrm{j}}$ and the polarization degree of $\lambda_{\mathrm{j}}$ is determined by the level structure $\alpha_{\mathcal{H}_{\mathrm{j}}}$, the tautological $\left(A_{\mathrm{j}}, \lambda_{\mathrm{j}}\right)$ over $\mathrm{M}_{\mathcal{H}_{\mathrm{j}}}$ defines (by forgetting the additional structures) a morphism from $\mathrm{M}_{\mathcal{H}_{\mathrm{j}}}$ to the 
Siegel moduli $\mathcal{A}_{g, d_{\mathrm{j}}}$ of genus $g=\frac{1}{2} \mathrm{rk}_{\mathbb{Z}}\left(L_{\mathrm{j}}\right)=\frac{1}{2} \mathrm{rk}_{\mathbb{Z}}(L)$ and polarization degree $d_{\mathrm{j}}^{2}=\left[L_{\mathrm{j}}^{\#}: L_{\mathrm{j}}\right]$, which induces a finite morphism

$$
\mathrm{M}_{\mathcal{H}_{\mathrm{j}}} \rightarrow \mathcal{A}_{g, d_{\mathrm{j}}} \underset{\mathbb{Z}}{\otimes \mathbb{Q}}
$$

by [30, Proposition 1.3.3.7, Corollary 2.2.2.8, and Proposition 2.2.2.9]. Similarly, the tautological $\left(A_{\mathrm{j}, \text { aux }}, \lambda_{\mathrm{j}, \text { aux }}\right)$ defines a morphism from $\mathrm{M}_{\mathcal{H}_{\mathrm{j}, \text { aux }}}$ to the Siegel moduli $\mathcal{A}_{g_{\mathrm{j}, \text { aux }}, d_{\mathrm{j}, \text { aux }}}$ of genus $g_{\mathrm{j} \text {,aux }}=\frac{1}{2} \mathrm{rk}_{\mathbb{Z}}\left(L_{\mathrm{j} \text {,aux }}\right)$ and (prime-to- $p$ ) polarization degree $d_{\mathrm{j}, \text { aux }}^{2}=\left[L_{\mathrm{j} \text {,aux }}^{\#}: L_{\mathrm{j} \text {,aux }}\right]$, which induces a finite morphism

$$
\mathrm{M}_{\mathcal{H}_{\mathrm{j}, \text { aux }}} \rightarrow \mathcal{A}_{g_{\mathrm{j}, \text { aux }}, d_{\mathrm{j}, \mathrm{aux}}} \underset{\mathbb{Z}}{\otimes} \mathbb{Z}_{(p)}
$$

As explained in the proof of Lemma 4.5, the construction as in (2) of Lemma 4.1 defines a finite morphism $\mathcal{A}_{g, d_{\mathrm{j}}} \rightarrow \mathcal{A}_{g_{\mathrm{j}, \text { aux }}, d_{\mathrm{j}, \mathrm{aux}}}$. By comparing the universal properties, the composition $\mathrm{M}_{\mathcal{H}_{\mathrm{j}}} \rightarrow \mathrm{M}_{\mathcal{H}_{\mathrm{j}, \text { aux }}} \otimes_{\mathbb{Z}} \mathbb{Q} \rightarrow \mathcal{A}_{g_{\mathrm{j}, \text { aux }}, d_{\mathrm{j}, \text { aux }}} \otimes_{\mathbb{Z}} \mathbb{Q}$ of finite morphisms coincides with the composition $\mathrm{M}_{\mathcal{H}_{\mathrm{j}}} \rightarrow \mathcal{A}_{g, d_{\mathrm{j}}} \otimes_{\mathbb{Z}} \mathbb{Q} \rightarrow \mathcal{A}_{g_{\mathrm{j} \text {,aux }}, d_{\mathrm{j}, \text { aux }}} \otimes_{\mathbb{Z}} \mathbb{Q}$ of finite morphisms. Since $\mathcal{A}_{g, d_{\mathrm{j}}} \rightarrow \mathcal{A}_{g_{\mathrm{j}, \text { aux }}, d_{\mathrm{j}, \text { aux }}}$ and $\mathrm{M}_{\mathcal{H}_{\mathrm{j}, \text { aux }}} \rightarrow \mathcal{A}_{g_{\mathrm{j}, \text { aux }}, d_{\mathrm{j}, \text { aux }}} \otimes_{\mathbb{Z}} \mathbb{Z}_{(p)}$ are finite, it follows that $\overrightarrow{\mathrm{M}}_{\mathcal{H}}$ is canonically isomorphic to the normalization of $\prod_{\mathrm{j} \in \mathrm{J}} \mathcal{A}_{g, d_{\mathrm{j}}} \otimes_{\mathbb{Z}} \mathbb{Z}_{(p)}$ under the canonical morphism $\mathrm{M}_{\mathcal{H}} \rightarrow \prod_{\mathrm{j} \in \mathrm{J}} \mathcal{A}_{g, d_{\mathrm{j}}} \otimes_{\mathbb{Z}} \mathbb{Z}_{(p)}$. In particular, for each $\mathrm{j} \in \mathrm{J}$, the tautological object $\left(A_{\mathrm{j}}, \lambda_{\mathrm{j}}\right)$ over $\mathrm{M}_{\mathcal{H}_{\mathrm{j}}} \cong \mathrm{M}_{\mathcal{H}}$ extends to an object $\left(\vec{A}_{\mathrm{j}}, \vec{\lambda}_{\mathrm{j}}\right)$ parameterized by the canonical morphism $\overrightarrow{\mathrm{M}}_{\mathcal{H}} \rightarrow \mathcal{A}_{g, d_{\mathrm{j}}}$ induced by the canonical morphism $\overrightarrow{\mathrm{M}}_{\mathcal{H}} \rightarrow \prod_{\mathrm{j} \in \mathrm{J}} \mathcal{A}_{g, d_{\mathrm{j}}}$. This also shows, as in the last paragraph of the statement of the proposition, that $\overrightarrow{\mathrm{M}}_{\mathcal{H}}$ is canonical and independent of the auxiliary choices.

Except for the quasiprojectivity of $\left[\overrightarrow{\mathrm{M}}_{\mathcal{H}}\right]$ over $\overrightarrow{\mathrm{S}}_{0}$, and for the ampleness of $\omega_{\overrightarrow{\mathrm{M}}_{\mathcal{H}}, \mathrm{J}}$ when $\mathcal{H}$ is neat, both of which will follow from Proposition 6.4 below, the remaining statements of the proposition are self-explanatory.

Although Proposition 6.1 is stated without any reference to compactifications, the easiest way to show the quasiprojectivity of $\left[\overrightarrow{\mathrm{M}}_{\mathcal{H}}\right]$ over $\overrightarrow{\mathrm{S}}_{0}$, and the ampleness of $\omega_{\overrightarrow{\mathrm{M}}_{\mathcal{H}}, \mathrm{J}}$ when $\mathcal{H}$ is neat, is to introduce the minimal compactifications (this is a natural consideration because this is what the minimal compactifications in [3] $\operatorname{did}$ over $\mathbb{C}$ ).

Proposition 6.4. Let $\overrightarrow{\mathrm{M}}_{\mathcal{H}}^{\min }$ denote the normalization of $\prod_{\mathrm{j} \in \mathrm{J}} \mathrm{M}_{\mathcal{H}_{\mathrm{j}, \text { aux }}}^{\min }$ in $\mathrm{M}_{\mathcal{H}}^{\min }$ under the morphism

$$
\mathrm{M}_{\mathcal{H}}^{\min } \rightarrow \prod_{\mathrm{j} \in \mathrm{J}} \mathrm{M}_{\mathcal{H}_{\mathrm{j}, \text { aux }}}^{\min }
$$


induced by (5.37). Then $\overrightarrow{\mathrm{M}}_{\mathcal{H}}^{\min }$ is a normal scheme projective and flat over $\overrightarrow{\mathrm{S}}_{0}=\operatorname{Spec}\left(\mathcal{O}_{F_{0},(p)}\right)$ equipped with a canonical isomorphism $\overrightarrow{\mathrm{M}}_{\mathcal{H}}^{\min } \times_{\overrightarrow{\mathrm{S}}_{0}} \mathrm{~S}_{0} \cong \mathrm{M}_{\mathcal{H}}^{\min }$ over $\mathrm{S}_{0}=\operatorname{Spec}\left(F_{0}\right)$, and with a canonical finite morphism

$$
\overrightarrow{\mathrm{M}}_{\mathcal{H}}^{\min } \rightarrow \prod_{\mathrm{j} \in \mathrm{J}} \mathrm{M}_{\mathcal{H}_{\mathrm{j}, \text { aux }}}^{\min }
$$

extending (5.37) and (6.5).

By construction, $\overrightarrow{\mathrm{M}}_{\mathcal{H}}$ is an open dense subscheme of $\overrightarrow{\mathrm{M}}_{\mathcal{H}}^{\min }$, because $\mathrm{M}_{\mathcal{H}_{\mathrm{j}, \text { aux }}}$ is an open dense subscheme of $\mathrm{M}_{\mathcal{H}_{\mathrm{j}, \text { aux }}}^{\text {min }}$ (by [30, Theorem 7.2.4.1], under the assumption that $\mathcal{H}$ and $\mathcal{H}_{\mathrm{j}, \text { aux }}$ are neat), for all $\mathrm{j} \in \mathrm{J}$.

For each $\mathrm{j} \in \mathrm{J}$, let $a_{\mathrm{j}, 0} \geqslant 1$ and $a_{\mathrm{j}} \geqslant 1$ be the integers as in Lemma 5.30, and let $a_{\mathrm{J}}:=\sum_{\mathrm{j} \in \mathrm{J}} a_{\mathrm{j}}$ as in Proposition 6.1. Then, for each $\mathrm{j} \in \mathrm{J}$, the invertible sheaf $\omega_{\mathrm{M}_{\mathcal{H}_{\mathrm{j}}}}^{\otimes a_{\mathrm{j}}}$ over $\mathrm{M}_{\mathcal{H}_{\mathrm{j}}} \cong \mathrm{M}_{\mathcal{H}}$ and the invertible sheaf $\omega_{\mathrm{M}_{\mathcal{H}_{\mathrm{j}}}^{\min }}^{\otimes a_{\mathrm{j}}}$ over $\mathrm{M}_{\mathcal{H}_{\mathrm{j}}}^{\min } \cong \mathrm{M}_{\mathcal{H}}^{\min }$ compatibly extend to an invertible sheaf over $\overrightarrow{\mathrm{M}}_{\mathcal{H}}^{\min }$, which we denote by $\omega_{\overrightarrow{\mathrm{M}}_{\mathcal{H}}, \mathrm{j}}^{\otimes a_{\mathrm{j}}}$ by abuse of notation, which is canonically isomorphic to the pullback of $\omega_{\mathrm{M}_{\mathcal{H}_{\mathrm{j}, \text { aux }}}^{m a_{\mathrm{j}, 0}}}^{\otimes}$ to $\overrightarrow{\mathrm{M}}_{\mathcal{H}}^{\min }$, whose pullback to $\overrightarrow{\mathrm{M}}_{\mathcal{H}}$ is canonically isomorphic to $\omega_{\overrightarrow{\mathrm{M}}_{\mathcal{H}}, \mathrm{j}}^{\otimes a_{\mathrm{j}}}$. Moreover, the invertible sheaf $\omega_{\mathrm{M}_{\mathcal{H}}}^{\otimes a_{\mathrm{J}}}$ extends to the invertible sheaf

$$
\omega_{\overrightarrow{\mathrm{M}}_{\mathcal{H}}^{\min }, \mathrm{J}}:=\bigotimes_{\mathrm{j} \in \mathrm{J}} \omega_{\overrightarrow{\mathrm{M}}_{\mathcal{H}}^{\min , \mathrm{j}}}^{\otimes a_{j}}
$$

over $\overrightarrow{\mathrm{M}}_{\mathcal{H}}^{\min }$, which is canonically isomorphic to the pullback of $\bigotimes_{\mathrm{j} \in \mathrm{J}} \omega_{\mathrm{M}_{\mathcal{H}_{\mathrm{j}, a x}}^{\min }}^{\otimes a_{\mathrm{j}, 0}}$ under (6.6). This $\omega_{\overrightarrow{\mathrm{M}}_{\mathcal{H}}^{\text {min }}, \mathrm{m}}$ is ample and induces a canonical isomorphism

$$
\overrightarrow{\mathrm{M}}_{\mathcal{H}}^{\min } \cong \operatorname{Proj}\left(\bigoplus_{k \geqslant 0} \Gamma\left(\overrightarrow{\mathrm{M}}_{\mathcal{H}}^{\min }, \omega_{\overrightarrow{\mathrm{M}}_{\mathcal{H}}^{\text {min }}, \mathrm{J}}^{\otimes k}\right)\right) .
$$

We obtain the same normalization $\overrightarrow{\mathrm{M}}_{\mathcal{H}}^{\mathrm{min}}$ (up to canonical isomorphism) satisfying the analogous properties if, for each $\mathrm{j} \in \mathrm{J}$, we replace $\mathcal{H}_{\mathrm{j}, \text { aux }}$ with any neat open compact subgroup of $\mathrm{G}_{\mathrm{j}, \text { aux }}\left(\hat{\mathbb{Z}}^{p}\right)$ still containing the image of $\mathcal{H}_{\mathrm{j}}$ under the homomorphism $\mathrm{G}_{\mathrm{j}}(\hat{\mathbb{Z}}) \rightarrow \mathrm{G}_{\mathrm{j}, \text { aux }}\left(\hat{\mathbb{Z}}^{p}\right)$ given by $(4.10)$.

As in the case of $\vec{M}_{\mathcal{H}}$ in Proposition 6.1, it is also true that, up to canonical isomorphism, $\overrightarrow{\mathrm{M}}_{\mathcal{H}}^{\mathrm{min}}$ depends only on the choices of the linear algebraic data in Section 2, but not on the auxiliary choices in Sections 4 and 5. However, the proof of this is somewhat indirect and will be postponed until Corollary 12.7 below. 
Proof of Proposition 6.4. By construction as a normalization, $\overrightarrow{\mathrm{M}}_{\mathcal{H}}^{\mathrm{min}}$ is normal and equipped with the finite morphism (6.6). For each $\mathrm{j} \in \mathrm{J}$, let us define the common extension $\omega_{\overrightarrow{\mathrm{M}}_{\mathcal{H}}^{\text {min }}, \mathrm{a}}^{\otimes a_{j}}$ of $\omega_{\mathrm{M}_{\mathcal{H}_{\mathrm{j}}}}^{\otimes a_{\mathrm{j}}}$ and $\omega_{\mathrm{M}_{\mathcal{H}_{\mathrm{j}}}^{\text {min }}}^{\otimes a_{\mathrm{j}}}$ to be the pullback of $\omega_{\mathrm{M}_{\mathcal{H}_{\mathrm{j}, \text { aux }}}^{\otimes m}}^{\otimes a_{\mathrm{j}, 0}}$ under the morphism $\overrightarrow{\mathrm{M}}_{\mathcal{H}}^{\text {min }} \rightarrow \mathrm{M}_{\mathcal{H}_{\mathrm{j}, \text { aux }}}^{\min }$ induced by (6.6), so that $\omega_{\overrightarrow{\mathrm{M}}_{\mathcal{H}}^{\min }, \mathrm{J}}=\bigotimes_{\mathrm{j} \in \mathrm{J}} \omega_{\overrightarrow{\mathrm{M}}_{\mathcal{H}}, \mathrm{ji}}^{\otimes a_{\mathrm{j}}}$ is the pullback of $\bigotimes_{\mathrm{j} \in \mathrm{J}} \omega_{\mathrm{M}_{\mathcal{H}_{\mathrm{j}, \text { aux }}}^{\text {min }}}^{\otimes a_{\mathrm{j}, 0}}$ under the finite morphism (6.6) (this is consistent with Lemma 5.30). Since $\omega_{\mathbf{M}_{\mathcal{H}_{j}, \text { aux }}^{\text {min }}}^{\otimes a_{\mathrm{j}, 0}}$ is ample over $\mathrm{M}_{\mathcal{H}_{\mathrm{j} \text {,aux }}}^{\min }$ for all $\mathrm{j} \in \mathrm{J}$, the pullback $\omega_{\overrightarrow{\mathrm{M}}_{\mathcal{H}}^{\text {min }}, \mathrm{J}}$ of $\bigotimes_{\mathrm{j} \in \mathrm{J}} \omega_{\mathrm{M}_{\mathcal{H}_{\mathrm{j}, \text { aux }}}^{\text {min }}}^{\otimes a_{\mathrm{j}} 0}$ under (6.6) is also ample. This shows, in particular, that $\overrightarrow{\mathrm{M}}_{\mathcal{H}}^{\text {min }}$ is projective over $\overrightarrow{\mathrm{S}}_{0}$. Since the structural sheaf of $\overrightarrow{\mathrm{M}}_{\mathcal{H}}^{\text {min }}$ is normal and hence has no $p$-torsion, it is also flat over $\vec{S}_{0}$. Since the pullback of $\bigotimes_{\mathrm{j} \in \mathrm{J}} \omega_{\mathrm{M}_{\mathrm{H} j \mathrm{j}, \text { aux }}^{\text {min }}}^{\otimes a_{\mathrm{j}}}$ to $\prod_{\mathrm{j} \in \mathrm{J}} \mathrm{M}_{\mathcal{H}_{\mathrm{j}, \text { aux }}}$ is canonically isomorphic to $\bigotimes_{\mathrm{j} \in \mathrm{J}} \omega_{\mathrm{M}_{\mathcal{H}}, \text { aux }}^{\otimes a_{j},}$, its further pullback to $\overrightarrow{\mathrm{M}}_{\mathcal{H}}$, which is canonically isomorphic to the pullback of $\omega_{\overrightarrow{\mathrm{M}}_{\mathcal{H}} \text { min }}$ by construction, is canonically isomorphic to $\omega_{\overrightarrow{\mathrm{M}}_{\mathcal{H}}, \mathrm{J}}$ (by the part of Proposition 6.1 we have proved). The remaining statements of the proposition are self-explanatory.

Now the proof of Proposition 6.1 is also complete.

REMARK 6.7. In our constructions (including ones to be give below), taking normalizations will never introduce pathologies, either because we are talking integral closures in (products of) separable field extensions (see [36, Section 33, Lemma 1]), or because the schemes in questions are all excellent (being a localization of a scheme of finite type over $\mathbb{Z}$; see [35, Sections 31-34] for more discussions).

For each stratum $Z_{\left[\left(\Phi_{\mathcal{H}}, \delta_{\mathcal{H}}\right)\right]}$ as in [30, Theorem 7.2.4.1(4)], consider its closure $\overline{\mathrm{Z}}_{\left[\left(\Phi_{\mathcal{H}}, \delta_{\mathcal{H}}\right)\right]}$ in $\mathrm{M}_{\mathcal{H}}^{\min }$ and its closure $\overrightarrow{\mathrm{Z}}_{\left[\left(\Phi_{\mathcal{H}}, \delta_{\mathcal{H}}\right)\right]}$ in $\overrightarrow{\mathrm{M}}_{\mathcal{H}}^{\min }$. Then we define a locally closed subscheme

$$
\begin{aligned}
& \overrightarrow{\mathrm{Z}}_{\left[\left(\Phi_{\mathcal{H}}, \delta_{\mathcal{H}}\right)\right]}:=\overrightarrow{\bar{Z}}_{\left[\left(\Phi_{\mathcal{H}}, \delta_{\mathcal{H}}\right)\right]}-\quad \bigcup \quad \overrightarrow{\mathrm{Z}}_{\left[\left(\Phi_{\mathcal{H}}^{\prime}, \delta_{\mathcal{H}}^{\prime}\right)\right]} \\
& \mathrm{Z}_{\left[\left(\Phi_{\mathcal{H}}, \delta_{\mathcal{H}}\right)\right]} \nsubseteq \overline{\mathrm{Z}}_{\left[\left(\Phi_{\mathcal{H}}^{\prime}, \delta_{\mathcal{H}}^{\prime}\right)\right]}
\end{aligned}
$$

of $\overrightarrow{\mathrm{M}}_{\mathcal{H}}^{\min }$. By definition, we have the following lemma.

LEMMA 6.9. If $\mathbf{Z}_{\left[\left(\Phi_{\mathcal{H}}, \delta_{\mathcal{H}}\right)\right]}$ is contained in the closure $\bar{Z}_{\left[\left(\Phi_{\mathcal{H}}^{\prime}, \delta_{\mathcal{H}}^{\prime}\right)\right]}$ of $\mathbf{Z}_{\left[\left(\Phi_{\mathcal{H}}^{\prime}, \delta_{\mathcal{H}}^{\prime}\right)\right]}$, then $\vec{Z}_{\left[\left(\Phi_{\mathcal{H}}, \delta_{\mathcal{H}}\right)\right]}$ is contained in $\vec{Z}_{\left[\left(\Phi_{\mathcal{H}}^{\prime}, \delta_{\mathcal{H}}^{\prime}\right)\right]}$, and the latter agrees with the closure of $\vec{Z}_{\left[\left(\Phi_{\mathcal{H}}^{\prime}, \delta_{\mathcal{H}}^{\prime}\right)\right]}$. 
REMARK 6.10. We shall call $\vec{Z}_{\left[\left(\Phi_{\mathcal{H}}, \delta_{\mathcal{H}}\right)\right]}$ the $\left[\left(\Phi_{\mathcal{H}}, \delta_{\mathcal{H}}\right)\right]$-stratum from now on, although we will have to wait until Theorems 12.1 and 12.16 below to see that it does satisfy the familiar properties as in [30, Theorem 7.2.4.1(4) and (5)].

\section{Toroidal compactifications defined by normalization}

For each $\mathrm{j} \in \mathrm{J}$, and for each $\left(\mathrm{Z}_{\mathcal{H}_{\mathrm{j}}}, \Phi_{\mathcal{H}_{\mathrm{j}}}, \delta_{\mathcal{H}_{\mathrm{j}}}\right)$ inducing $\left(\mathrm{Z}_{\mathcal{H}_{\mathrm{j}, \text { aux }}}, \Phi_{\mathcal{H}_{\mathrm{j}, \text { aux }}}, \delta_{\mathcal{H}_{\mathrm{j}, \text { aux }}}\right)$ as in (5.8), we have a boundary version

$$
\mathrm{M}_{\mathcal{H}_{\mathrm{j}}}^{\mathrm{Z} \mathcal{H}_{\mathrm{j}}} \rightarrow \mathrm{M}_{\mathcal{H}_{\mathrm{j}, \text { aux }}}^{\mathrm{Z} \mathcal{H}_{\mathrm{j} \text { aux }}} \underset{\mathbb{Z}}{\otimes} \mathbb{Q}
$$

of (4.14) (see [30, Definition 5.4.2.6 and the errata]), whose composition with (3.2) gives the boundary version

$$
\mathrm{M}_{\mathcal{H}}^{\mathrm{Z} \mathcal{H}} \rightarrow \mathrm{M}_{\mathcal{H}_{\mathrm{j}, \text { aux }}}^{\mathrm{Z} \mathcal{H}_{\mathrm{j} \text { aux }}} \underset{\mathbb{Z}}{\otimes} \mathbb{Q}
$$

of (4.13). These morphisms (7.2), for all $\mathrm{j} \in \mathrm{J}$, induce the boundary version

$$
\mathrm{M}_{\mathcal{H}}^{\mathrm{ZH}} \rightarrow \prod_{\mathrm{j} \in \mathrm{J}} \mathrm{M}_{\mathcal{H}_{\mathrm{j}, \text { aux }}}^{\mathrm{Z}_{\mathcal{H}_{\mathrm{j}} \text {,ax }}} \otimes_{\mathbb{Z}} \mathbb{Q}
$$

of (4.15).

Proposition 7.4. Let $\overrightarrow{\mathrm{M}}_{\mathcal{H}}^{Z_{\mathcal{H}}}$ denote the normalization of $\prod_{\mathrm{j} \in \mathrm{J}} \mathrm{M}_{\mathcal{H}_{\mathrm{j}, \text { aux }}}^{\mathrm{Z}_{\mathcal{H}_{\mathrm{j}} \text { aux }}}$ in $\mathrm{M}_{\mathcal{H}}^{Z_{\mathcal{H}}}$ under the morphism

$$
\mathrm{M}_{\mathcal{H}}^{Z_{\mathcal{H}}} \rightarrow \prod_{\mathrm{j} \in \mathrm{J}} \mathrm{M}_{\mathcal{H}_{\mathrm{j} \text {,aux }}^{Z_{\mathrm{j}} \text {,aux }}}^{Z}
$$

induced by (7.3), which is equipped with a canonical finite morphism

$$
\overrightarrow{\mathrm{M}}_{\mathcal{H}}^{\mathrm{ZH}} \rightarrow \prod_{\mathrm{j} \in \mathrm{J}} \mathrm{M}_{\mathcal{H}_{\mathrm{j}, \text { aux }}^{\mathrm{Z}}}^{\mathrm{H}_{\mathrm{j}, \text { aux }}}
$$

compatible with (7.3) and (7.5). Then the tautological tuple $\left(B_{\mathrm{j}}, \lambda_{B_{\mathrm{j}}}, i_{B_{\mathrm{j}}}, \varphi_{-1, \mathcal{H}_{\mathrm{j}}}\right)$ over $\mathrm{M}_{\mathcal{H}_{\mathrm{j}}}^{Z_{\mathcal{H}_{\mathrm{j}}}} \cong \mathrm{M}_{\mathcal{H}}^{Z_{\mathcal{H}}}$ extends to a degenerating family $\left(\vec{B}_{\mathrm{j}}, \lambda_{\vec{B}_{\mathrm{j}}}, i_{\vec{B}_{\mathrm{j}}}, \vec{\varphi}_{-1, \mathcal{H}_{\mathrm{j}}}\right)$ over $\overrightarrow{\mathrm{M}}_{\mathcal{H}}^{\mathrm{Z}}$, where $\vec{\varphi}_{-1, \mathcal{H}_{\mathrm{j}}}$ is defined only over $\mathrm{M}_{\mathcal{H}_{\mathrm{j}}}^{\mathrm{H}_{\mathrm{j}}} \cong \mathrm{M}_{\mathcal{H}}^{\mathrm{Z} \mathcal{H}}$ (cf. Proposition 6.1). Up to canonical isomorphism, $\overrightarrow{\mathrm{M}}_{\mathcal{H}}^{\mathrm{Z}_{\mathcal{H}}}$ does not depend on the precise choices of $\left\{\mathcal{H}_{\mathrm{j} \text {, aux }}\right\}_{\mathrm{j} \in \mathrm{J}}$ (we will omit such justifications for similar constructions later).

Proof. The proof is similar to the one for $\overrightarrow{\mathrm{M}}_{\mathcal{H}}$ in Proposition 6.1. 
For each $\mathrm{j} \in \mathrm{J}$, suppose $\Sigma_{\mathrm{j} \text {,aux }}=\left\{\Sigma_{\Phi_{\mathcal{H}_{\mathrm{j}, \text { aux }}}}\right\}_{\left[\left(\Phi_{\mathcal{H}_{\mathrm{j}, \text { aux }},}, \mathcal{H}_{\mathcal{H}_{\mathrm{j}, \text { aux }}}\right)\right]}$ is a compatible choice of admissible smooth rational polyhedral cone decomposition data for $\mathrm{M}_{\mathcal{H}_{\mathrm{j} \text {,aux }}}$ as

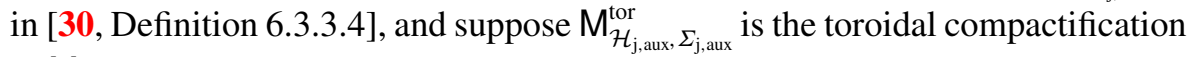
of $\mathrm{M}_{\mathcal{H}_{\mathrm{j}, \text { aux }}}$ as in [30, Theorem 6.4.1.1]. For simplicity, we shall assume that $\Sigma_{\mathrm{j} \text {,aux }}$ is projective as in [30, Definition 7.3.1.3], so that (under the assumption that $\mathcal{H}_{\mathrm{j}, \text { aux }}$ is neat) $\mathrm{M}_{\mathcal{H}_{\mathrm{j}, \text { aux },}, \Sigma_{\mathrm{j}, \text { aux }}}^{\text {tor }}$ is a scheme projective and smooth over $\operatorname{Spec}\left(\mathcal{O}_{F_{0, \mathrm{j}, \text { aux }},(p)}\right)(\operatorname{see}[\mathbf{3 0}$, Theorem 7.3.3.4]).

For each representative $\left(\mathrm{Z}_{\mathcal{H}}, \Phi_{\mathcal{H}}, \delta_{\mathcal{H}}\right)$ of cusp label of $\mathrm{M}_{\mathcal{H}}$, we define a (possibly nonsmooth) rational polyhedral cone decomposition $\Sigma_{\Phi_{\mathcal{H}}}$ of $\mathbf{P}_{\Phi_{\mathcal{H}}}$ by

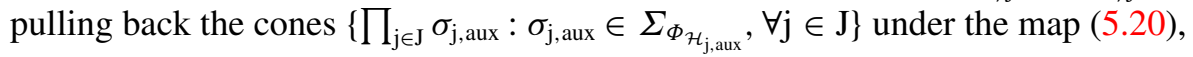
which satisfies [30, Condition 6.2.5.25] with respect to $\Gamma_{\Phi_{\mathcal{H}}}$ (so that the analog of [30, Lemma 6.2.5.27] applies) when each $\Sigma_{\Phi_{\mathcal{H}_{\mathrm{j}, \text { aux }}}}$ satisfies [30, Condition 6.2.5.25] with respect to $\Phi_{\mathcal{H}_{\mathrm{j}, \text { aux }}}$; and we define a compatible choice $\Sigma$ of (possibly nonsmooth) admissible rational polyhedral cone decomposition data for $\mathrm{M}_{\mathcal{H}}$ by having $\left(\mathrm{Z}_{\mathcal{H}}, \Phi_{\mathcal{H}}, \delta_{\mathcal{H}}\right)$ run through all such representatives (although we have only considered smooth cone decompositions in [30, Definition 6.3.3.4], the definition naturally generalizes to the case of nonsmooth cone decompositions). For each $\mathrm{j} \in \mathrm{J}$, the compatible choice $\Sigma$ for $\mathrm{M}_{\mathcal{H}}$ also induces a compatible choice $\Sigma_{\mathrm{j}}$ for $\mathrm{M}_{\mathcal{H}_{\mathrm{j}}}$, as in Lemma 3.21.

Let $\overrightarrow{\mathrm{M}}_{\mathcal{H}, \Sigma}^{\text {tor }}$ denote the normalization of $\prod_{\mathrm{j} \in \mathrm{J}} \mathrm{M}_{\mathcal{H}_{\mathrm{j}, \text { aux }, \Sigma_{\mathrm{j}, \text { aux }}}^{\text {tor }}}$ in $\mathrm{M}_{\mathcal{H}}$ under the morphism

$$
\mathrm{M}_{\mathcal{H}} \rightarrow \prod_{\mathrm{j} \in \mathrm{J}} \mathrm{M}_{\mathcal{H}_{\mathrm{j}, \text { aux }, \Sigma_{\mathrm{j}, \text { aux }}}^{\text {tor }}}
$$

induced by (6.2) and by the canonical morphisms $\mathrm{M}_{\mathcal{H}_{\mathrm{j}, \text { aux }}} \rightarrow \mathrm{M}_{\mathcal{H}_{\mathrm{j}, \text { aux }}, \Sigma_{\mathrm{j}, \text { aux }}}^{\text {tor }}$, for all $\mathrm{j} \in \mathrm{J}$, which is naturally a scheme over $\overrightarrow{\mathrm{S}}_{0}=\operatorname{Spec}\left(\mathcal{O}_{F_{0},(p)}\right)$ and equipped with a canonical finite morphism

$$
\overrightarrow{\mathrm{M}}_{\mathcal{H}, \Sigma}^{\text {tor }} \rightarrow \prod_{\mathrm{j} \in \mathrm{J}} \mathrm{M}_{\mathcal{H}_{\mathrm{j}, \text { aux }, \Sigma_{\mathrm{j}, \text { aux }}}^{\text {tor }}}
$$

compatible with (6.2) and (7.7) (this is similar to the considerations in, for example, [37], [59], [38], [25], and [34], although they have not explicitly considered a product of auxiliary toroidal compactifications as we do). A priori, this is an abuse of notation, because the definition uses $\left\{\Sigma_{\mathrm{j}, \text { aux }}\right\}_{\mathrm{j} \in \mathrm{J}}$ rather than the induced $\Sigma$ (nevertheless, we will justify this in Theorem 7.14 below).

LEMMA 7.9. With the setting as above, the scheme $\overrightarrow{\mathrm{M}}_{\mathcal{H}, \Sigma}^{\text {tor }}$ is proper and flat over $\overrightarrow{\mathrm{S}}_{0}$. Moreover, the morphisms (6.2) and (7.7) induce a canonical open immersion

$$
\overrightarrow{\mathrm{M}}_{\mathcal{H}} \rightarrow \overrightarrow{\mathrm{M}}_{\mathcal{H}, \Sigma}^{\text {tor }}
$$


and the image of (7.10) is dense in $\overrightarrow{\mathrm{M}}_{\mathcal{H}, \Sigma}^{\text {tor }}$ and coincides with the preimage of $\prod_{\mathrm{j} \in \mathrm{J}} \mathrm{M}_{\mathcal{H}_{\mathrm{j}, \text { aux }}}$ under the canonical morphism (7.8). Consequently, we may and we shall identify $\overrightarrow{\mathrm{M}}_{\mathcal{H}}$ with its image under (7.10), and view it as an open dense subscheme of $\overrightarrow{\mathrm{M}}_{\mathcal{H}, \Sigma}^{\text {tor }}$.

Proof. The proper flatness of $\overrightarrow{\mathrm{M}}_{\mathcal{H}, \Sigma}^{\text {tor }}$ over $\overrightarrow{\mathrm{S}}_{0}$ follows from the construction of $\overrightarrow{\mathrm{M}}_{\mathcal{H}, \Sigma}^{\text {tor }}$ by normalization, and from the proper smoothness of $\mathrm{M}_{\mathcal{H}_{\mathrm{j}, \text { aux }}, \Sigma_{\mathrm{j}, \text { aux }}}^{\text {to }}$ over $\operatorname{Spec}\left(\mathcal{O}_{F_{0, \mathrm{j}, \text { aux }},(p)}\right)$ for each $\mathrm{j} \in \mathrm{J}$. The remaining statements of the lemma follow from the construction of $\vec{M}_{\mathcal{H}}$ by normalization (see Proposition 6.1) and from Zariski's main theorem (see [14, III-1, 4.4.3, 4.4.11]).

Proposition 7.11 (Cf. [30, Theorem 7.2.4.1(3)]). Consider the canonical morphism

$$
\widehat{\oint}_{\mathcal{H}}: \overrightarrow{\mathrm{M}}_{\mathcal{H}, \Sigma}^{\text {tor }} \rightarrow \overrightarrow{\mathrm{M}}_{\mathcal{H}}^{\text {min }}
$$

induced by the canonical morphism $\oint_{\mathcal{H}_{\mathrm{j}, \text { aux }}}: \mathrm{M}_{\mathcal{H}_{\mathrm{j}, \text { aux }, \Sigma_{\mathrm{j}, \text { aux }}}^{\text {tor }}} \rightarrow \mathrm{M}_{\mathcal{H}_{\mathrm{j}, \text { aux }}}^{\min }$ for all $\mathrm{j} \in \mathrm{J}$ (by the constructions of $\overrightarrow{\mathrm{M}}_{\mathcal{H}, \Sigma}^{\text {tor }}$ and $\overrightarrow{\mathrm{M}}_{\mathcal{H}}^{\min }$ as normalizations; see Proposition 6.4). Let $\omega_{\overrightarrow{\mathrm{M}}_{\mathcal{H}, \Sigma}^{\text {tor }}}$ denote the pullback of $\omega_{\overrightarrow{\mathrm{M}}_{\mathcal{H}}^{\text {min }}, \mathrm{J}}$ (see Proposition 6.4). Then $\omega_{\overrightarrow{\mathrm{M}}_{\mathcal{H}, \Sigma}^{\text {tor }}}$ is canonically isomorphic to the pullback of $\bigotimes_{\mathrm{j} \in \mathrm{J}} \omega_{\mathrm{M}_{\mathcal{H}_{\mathrm{j}, \text { aux }} \Sigma_{\mathrm{j}, \text { aux }}}^{\otimes a_{\mathrm{j}, 0}}}$ under (7.8), and the canonical morphism

$$
\overrightarrow{\mathrm{M}}_{\mathcal{H}, \Sigma}^{\text {tor }} \rightarrow \operatorname{Proj}\left(\bigoplus_{k \geqslant 0} \Gamma\left(\overrightarrow{\mathrm{M}}_{\mathcal{H}, \Sigma}^{\text {tor }}, \omega_{\overrightarrow{\mathrm{M}}_{\mathcal{H}, \Sigma}^{\text {tor }}, \mathrm{J}}^{\otimes k}\right)\right)
$$

induces a canonical isomorphism

$$
\overrightarrow{\mathrm{M}}_{\mathcal{H}}^{\text {min }} \cong \operatorname{Proj}\left(\bigoplus_{k \geqslant 0} \Gamma\left(\overrightarrow{\mathrm{M}}_{\mathcal{H}, \Sigma}^{\text {tor }}, \omega_{\overrightarrow{\mathrm{M}}_{\mathcal{H}, \Sigma}^{\text {tor }}, \mathrm{J}}^{\otimes k}\right)\right)
$$

(compatible with the canonical morphism (7.12)).

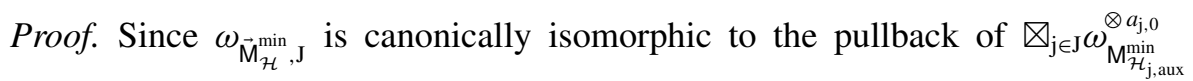
under (6.6) (see Proposition 6.4), and since $\omega_{\mathrm{M}_{\mathcal{H}_{\mathrm{j}, \text { aux }} \Sigma_{\mathrm{j}, \text { aux }}}^{\text {tor }}}$ is canonically isomorphic to the pullback of $\omega_{\mathrm{M}_{\mathcal{H}_{\mathrm{j}, \text { aux }}}^{\min }}$ to $\mathrm{M}_{\mathcal{H}_{\mathrm{j} \text {,aux }}, \Sigma_{\mathrm{j} \text {,aux }}}^{\text {tor }}$, for each $\mathrm{j} \in \mathrm{J}$, it follows that $\omega_{\overline{\mathrm{M}}_{\mathcal{H}, \Sigma}^{\text {tor }}, \mathrm{J}}$ is canonically isomorphic to the pullback of $\bigotimes_{\mathrm{j} \in \mathrm{J}} \omega_{\mathrm{M}_{\mathcal{H}}^{\mathrm{tor}}}^{\otimes a_{\mathrm{j}, \text { aux }}, \Sigma_{\mathrm{j}, \text { aux }}}$ under (7.8). 
Since (7.12) is an isomorphism over $\vec{M}_{\mathcal{H}}$ (see Lemma 7.9), the pullback of $\omega_{\overrightarrow{\mathrm{M}}_{\mathcal{H}, \Sigma}^{\text {tor }}, \mathrm{J}}$ to $\overrightarrow{\mathrm{M}}_{\mathcal{H}}$ is ample, and hence we can identify $\overrightarrow{\mathrm{M}}_{\mathcal{H}}$ with an open subscheme of $\operatorname{Proj}\left(\bigoplus_{k \geqslant 0} \Gamma\left(\overrightarrow{\mathrm{M}}_{\mathcal{H}, \Sigma}^{\text {tor }}, \omega_{\overrightarrow{\mathrm{M}}_{\mathcal{H}, \Sigma}^{\text {tor }}}^{\otimes k}\right)\right)$, which is necessarily dense because $\overrightarrow{\mathrm{M}}_{\mathcal{H}}$ is open and dense in $\overrightarrow{\mathrm{M}}_{\mathcal{H}, \Sigma}^{\text {tor }}$. Since $\omega_{\overrightarrow{\mathrm{M}}_{\mathcal{H}, \Sigma}^{\text {tor }}}$ descends to an ample invertible sheaf over Proj $\left(\bigoplus_{k \geqslant 0} \Gamma\left(\overrightarrow{\mathrm{M}}_{\mathcal{H}, \Sigma}^{\text {tor }}, \omega_{\overrightarrow{\mathrm{M}}_{\mathcal{H}, \Sigma}^{\text {tor }}, \mathrm{J}}^{\otimes k}\right)\right)$, the induced canonical proper morphism (see Proposition 6.4)

$$
\operatorname{Proj}\left(\bigoplus_{k \geqslant 0} \Gamma\left(\overrightarrow{\mathrm{M}}_{\mathcal{H}, \Sigma}^{\text {tor }}, \omega_{\overrightarrow{\mathrm{M}}_{\mathcal{H}, \Sigma}^{\text {tor }}, \mathrm{J}}^{\otimes k}\right)\right) \rightarrow \overrightarrow{\mathrm{M}}_{\mathcal{H}}^{\min } \cong \operatorname{Proj}\left(\bigoplus_{k \geqslant 0} \Gamma\left(\overrightarrow{\mathrm{M}}_{\mathcal{H}}^{\min }, \omega_{\overrightarrow{\mathrm{M}}_{\mathcal{H}}^{\min , \mathrm{J}}}^{\otimes k}\right)\right)
$$

is finite (see [14, II, 5.1.6, and III-1, 4.4.2]), which induces the identity morphism on $\overrightarrow{\mathrm{M}}_{\mathcal{H}}$ by restriction. Since $\overrightarrow{\mathrm{M}}_{\mathcal{H}}^{\text {min }}$ is noetherian and normal, (7.13) is an isomorphism by Zariski's main theorem (see [14, III-1, 4.4.3, 4.4.11]), as desired.

THEOREM 7.14 (Cf. [30, Theorem 6.4.1.1(6)]). With the setting as above, let $S$ be an irreducible noetherian normal scheme over $\overrightarrow{\mathrm{S}}_{0}=\operatorname{Spec}\left(\mathcal{O}_{F_{0},(p)}\right)$, with generic point $\eta$, which is equipped with a morphism

$$
\eta \rightarrow \mathrm{M}_{\mathcal{H}}
$$

Let $\left(A_{\eta}, \lambda_{\eta}, i_{\eta}, \alpha_{\mathcal{H}, \eta}\right)$ denote the pullback of the tautological object of $\mathrm{M}_{\mathcal{H}}$ to $\eta$ under (7.15). Suppose that, for each $\mathrm{j} \in \mathrm{J}$, we have a degenerating family

$$
\left(G_{\mathrm{j}, \text { aux }}^{\dagger}, \lambda_{\mathrm{j}, \text { aux }}^{\dagger}, i_{\mathrm{j}, \text { aux }}^{\dagger}, \alpha_{\mathcal{H}_{\mathrm{j}, \text { aux }}}^{\dagger}\right)
$$

of type $\mathrm{M}_{\mathcal{H}_{\mathrm{j}, \text { aux }}}$ over $S$, whose pullback

$$
\left(G_{\mathrm{j}, \text { aux }, \eta}, \lambda_{\mathrm{j}, \text { aux }, \eta}, i_{\mathrm{j}, \text { aux }, \eta}, \alpha_{\mathcal{H}_{\mathrm{j}, \text { aux }, \eta}}\right)
$$

to $\eta$ defines a morphism

$$
\eta \rightarrow \mathrm{M}_{\mathcal{H}_{\mathrm{j}, \text { aux }}}
$$

by the universal property of $\mathrm{M}_{\mathcal{H}_{\mathrm{j}, \mathrm{aux}}}$. These morphisms (7.16), for all $\mathrm{j} \in \mathrm{J}$, induce a morphism

$$
\eta \rightarrow \prod_{\mathrm{j} \in \mathrm{J}} \mathrm{M}_{\mathcal{H}_{\mathrm{j}, \text { aux }}} .
$$

Suppose moreover that (7.17) is the composition of (7.15) with the morphism (6.2) induced by (4.15). Then (7.15) (necessarily uniquely) extends to a morphism

$$
S \rightarrow \overrightarrow{\mathrm{M}}_{\mathcal{H}, \Sigma}^{\text {tor }}
$$

if and only if the following condition is satisfied at each geometric point $\bar{s}$ of $S$ : 
Consider any dominant morphism $\operatorname{Spec}(V) \rightarrow S$ centered at $\bar{s}$, where $V$ is a complete discrete valuation ring with fraction field $K$, algebraically closed residue field $k$, and discrete valuation $v$. By the semistable reduction theorem (see, for example, [30, Theorem 3.3.2.4]), up to replacing $K$ with a finite extension field and replacing $V$ accordingly, we may assume that the pullback of $A_{\eta}$ to $\operatorname{Spec}(K)$ extends to a semiabelian scheme $G^{\ddagger}$ over $\operatorname{Spec}(V)$. By the theory of Néron models (see [4]; cf. [52, IX, 1.4], [10, Ch. I, Proposition 2.7], or [30, Proposition 3.3.1.5]), the pullback of $\left(A_{\eta}, \lambda_{\eta}, i_{\eta}, \alpha_{\mathcal{H}, \eta}\right)$ to $\operatorname{Spec}(K)$ extends to a degenerating family

$$
\left(G^{\ddagger}, \lambda^{\ddagger}, i^{\ddagger}, \alpha_{\mathcal{H}}^{\ddagger}\right)
$$

of type $\mathrm{M}_{\mathcal{H}}$ over $\operatorname{Spec}(V)$, where $\alpha_{\mathcal{H}}^{\ddagger}$ is defined only over $\operatorname{Spec}(K)$, which defines an object of $\mathrm{DEG}_{\mathrm{PEL}, \mathrm{M}_{\mathcal{H}}}(V)$ corresponding to a tuple

$$
\left(B^{\ddagger}, \lambda_{B^{\ddagger}}, i_{B^{\ddagger}}, \underline{X^{\ddagger}}, \underline{Y^{\ddagger}}, \phi^{\ddagger}, c^{\ddagger}, c^{\vee, \ddagger}, \tau^{\ddagger},\left[\alpha_{\mathcal{H}}^{\natural, \ddagger}\right]\right)
$$

in $\mathrm{DD}_{\mathrm{PEL}, \mathrm{M}_{\mathcal{H}}}(V)$ under [30, Theorem 5.3.1.19].

Then we have a fully symplectic liftable admissible filtration $\mathrm{Z}_{\mathcal{H}}^{+}$determined by $\left[\alpha_{\mathcal{H}}^{\natural, \ddagger}\right]$. Moreover, the étale sheaves $\underline{X}^{\ddagger}$ and $\underline{Y}^{\ddagger}$ are necessarily constant, because the base ring $V$ is strict local. Hence it makes sense to say we also have a uniquely determined torus argument $\Phi_{\mathcal{H}}^{+}$at level $\mathcal{H}$ for $\mathrm{Z}_{\mathcal{H}}^{+}$.

On the other hand, we have objects $\underline{\Phi}_{\mathcal{H}}\left(G^{\ddagger}\right), \underline{\mathbf{S}}_{\underline{\mathcal{H}}_{\mathcal{H}}\left(G^{\ddagger}\right)}$, and $\underline{B}\left(G^{\ddagger}\right)$ (see [30, Construction 6.3.1.1]), which define objects $\Phi_{\mathcal{H}}^{\ddagger}, \mathbf{S}_{\Phi_{\mathcal{H}}^{\ddagger}}$, and, in particular,

$$
B^{\ddagger}: \mathbf{S}_{\Phi_{\mathcal{H}}^{\ddagger}} \rightarrow \operatorname{Inv}(V)
$$

over the special fiber. Then

$$
v \circ B^{\ddagger}: \mathbf{S}_{\Phi_{\mathcal{H}}^{\ddagger}} \rightarrow \mathbb{Z}
$$

defines an element of $\mathbf{S}_{\Phi_{\mathcal{H}}^{\ddagger}}^{\vee}$, where $v: \operatorname{Inv}(V) \rightarrow \mathbb{Z}$ is the homomorphism induced by the discrete valuation of $V$.

Then the condition is that, for each $\operatorname{Spec}(V) \rightarrow S$ as above (centered at $\bar{s}$ ), and for some (and hence every) choice of $\delta_{\mathcal{H}}^{\ddagger}$, there is a cone $\sigma^{\ddagger}$ in the cone decomposition $\Sigma_{\Phi_{\mathcal{H}}^{\ddagger}}$ of $\mathbf{P}_{\Phi_{\mathcal{H}}^{\ddagger}}$ such that $\bar{\sigma}^{\ddagger}$ contains all $v \circ B^{\ddagger}$ obtained in this way (as explained in the proof of [30, Proposition 6.3.3.11], we may assume that $\sigma^{\ddagger}$ is minimal among such choices; also, it follows from the positivity of $\tau^{\ddagger}$ that $\left.\sigma^{\ddagger} \subset \mathbf{P}_{\Phi_{\mathcal{H}}^{\ddagger}}^{+}\right)$.

In particular, since this condition involves only $\Sigma$, it follows that the scheme $\overrightarrow{\mathrm{M}}_{\mathcal{H}, \Sigma}^{\text {tor }}$ depends (up to canonical isomorphism) only on $\Sigma$, but not the choice of the $\left\{\Sigma_{\mathrm{j}, \text { aux }}\right\}_{\mathrm{j} \in \mathrm{J}}$ inducing $\Sigma$. 
Proof. Let $\operatorname{Spec}(V) \rightarrow S$ be any morphism as in the statement of the proposition.

For each $\mathrm{j} \in \mathrm{J}$, let $\left(A_{\mathrm{j}, \eta}, \lambda_{\mathrm{j}, \eta}, i_{\mathrm{j}, \eta}, \alpha_{\mathcal{H}_{\mathrm{j}}, \eta}\right)$ denote the pullback of the tautological object of $\mathrm{M}_{\mathcal{H}_{\mathrm{j}}}$ to $\eta$ under the composition of (7.15) with (2.1). Since $\left(A_{\eta}\right.$, $\left.\lambda_{\eta}, i_{\eta}, \alpha_{\mathcal{H}, \eta}\right)$ induces $\left(A_{\mathrm{j}, \eta}, \lambda_{\mathrm{j}, \eta}, i_{\mathrm{j}, \eta}, \alpha_{\mathcal{H}_{\mathrm{j}, \eta}}\right)$ via a $\mathbb{Q}^{\times}$-isogeny, by the theory of Néron models, the pullback of $\left(A_{\mathrm{j}, \eta}, \lambda_{\mathrm{j}, \eta}, i_{\mathrm{j}, \eta}, \alpha_{\mathcal{H}_{\mathrm{j}, \eta}}\right)$ to $\operatorname{Spec}(K)$ also extends to a degenerating family

$$
\left(G_{\mathrm{j}}^{\ddagger}, \lambda_{\mathrm{j}}^{\ddagger}, i_{\mathrm{j}}^{\ddagger}, \alpha_{\mathcal{H}_{\mathrm{j}}}^{\ddagger}\right)
$$

of type $\mathrm{M}_{\mathcal{H}_{\mathrm{j}}}$ over $\operatorname{Spec}(V)$, where $\alpha_{\mathcal{H}_{\mathrm{j}}}^{\ddagger}$ is defined only over $\operatorname{Spec}(K)$, which defines an object of $\mathrm{DEG}_{\mathrm{PEL}, \mathrm{M}_{\mathcal{H}_{\mathrm{j}}}}(V)$ corresponding to a tuple

$$
\left(B_{\mathrm{j}}^{\ddagger}, \lambda_{B_{\mathrm{j}}^{\ddagger}}, i_{B_{\mathrm{j}}^{\ddagger}}, \underline{X_{\mathrm{j}}^{\ddagger}}, \underline{Y}_{\mathrm{j}}^{\ddagger}, \phi_{\mathrm{j}}^{\ddagger}, c_{\mathrm{j}}^{\ddagger}, c_{\mathrm{j}}^{\vee, \ddagger}, \tau_{\mathrm{j}}^{\ddagger},\left[\alpha_{\mathcal{H}_{\mathrm{j}}}^{\natural, \ddagger}\right]\right)
$$

in $\mathrm{DD}_{\mathrm{PEL}, \mathrm{M}_{\mathcal{H}_{\mathrm{j}}}}(V)$ under [30, Theorem 5.3.1.19]; moreover, we may and we shall assume that the cusp label determined by the object in $\operatorname{DD}_{\mathrm{PEL}, \mathrm{M}_{\mathcal{H}_{\mathrm{j}}}}(V)$ is represented by the $\left(\mathrm{Z}_{\mathcal{H}_{\mathrm{j}}}^{\ddagger}, \Phi_{\mathcal{H}_{\mathrm{j}}}^{\ddagger}, \delta_{\mathcal{H}_{\mathrm{j}}}^{\ddagger}\right)$ assigned to $\left(\mathrm{Z}_{\mathcal{H}}^{\ddagger}, \Phi_{\mathcal{H}}^{\ddagger}, \delta_{\mathcal{H}}^{\ddagger}\right)$ via (3.8), and that the induced morphism $v \circ B_{\mathrm{j}}^{\ddagger} \in \mathbf{S}_{\Phi_{\mathcal{H}_{j}}^{\ddagger}}^{\vee}$ is a $\mathbb{Q}_{>0}^{\times}$-multiple of the image of $v \circ B^{\ddagger} \in \mathbf{S}_{\Phi_{\mathcal{H}}^{\ddagger}}^{\vee}$ under (3.12) (see Lemma 3.16).

For each $\mathrm{j} \in \mathrm{J}$, let $\left(G_{\mathrm{j} \text {,aux }}^{\ddagger}, \lambda_{\mathrm{j}, \text { aux }}^{\ddagger}, i_{\mathrm{j} \text {,aux }}^{\ddagger}, \alpha_{\mathcal{H}_{\mathrm{j} \text {,au }}}^{\ddagger}\right)$ denote the pullback of the degenerating family $\left(G_{\mathrm{j}, \text { aux }}^{\dagger}, \lambda_{\mathrm{j}, \text { aux }}^{\dagger}, i_{\mathrm{j}, \text { aux }}^{\dagger}, \alpha_{\mathcal{H}_{\mathrm{j}, \text { aux }}}^{\dagger}\right)$ under the $\operatorname{Spec}(V) \rightarrow S$ above,

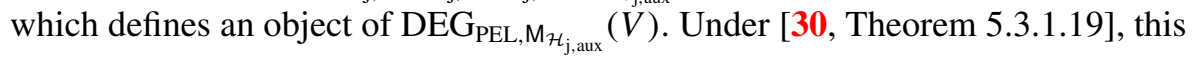
corresponds to an object of $\operatorname{DD}_{\mathrm{PEL}, \mathrm{M}_{\mathcal{H} \text {, aux }}}(V)$, which, in particular, determines a cusp label $\left[\left(\mathrm{Z}_{\mathcal{H}_{\mathrm{j}, \text { aux }}^{\ddagger}}^{\ddagger}, \Phi_{\mathcal{H}_{\mathrm{j} \text {,aux }}^{\ddagger}}^{\ddagger}, \delta_{\mathcal{H}_{\mathrm{j} \text {,aux }}}^{\ddagger}\right]\right.$ and an element $v \circ B_{\mathrm{j} \text {,aux }}^{\ddagger} \in \mathbf{S}_{\Phi_{\mathcal{H}_{\mathrm{j}} \text {,aux }}^{\ddagger}}^{\vee}$ for some representative $\left(\mathrm{Z}_{\mathcal{H}_{\mathrm{j}, \text { aux }}}^{\ddagger}, \Phi_{\mathcal{H}_{\mathrm{j}, \text { aux }}}^{\ddagger}, \delta_{\mathcal{H}_{\mathrm{j}} \text {,aux }}^{\ddagger}\right)$ of $\left[\left(\mathrm{Z}_{\mathcal{H}_{\mathrm{j}} \text {,aux }}^{\ddagger}, \Phi_{\mathcal{H}_{\mathrm{j}, \text { aux }}}^{\ddagger}, \delta_{\mathcal{H}_{\mathrm{j}, \text { aux }}}^{\ddagger}\right)\right]$. By the construction of (4.15), the assumption that (7.17) is the composition of (7.15) with the morphism (6.2) induced by (4.15) means that $\left(G_{\mathrm{j}, \text { aux }, \eta}, \lambda_{\mathrm{j}, \text { aux }, \eta}, i_{\mathrm{j}, \text { aux }, \eta}, \alpha_{\mathcal{H}_{\mathrm{j}, \text { aux }, \eta}}\right)$ is induced by $\left(A_{\mathrm{j}, \eta}, \lambda_{\mathrm{j}, \eta}, i_{\mathrm{j}, \eta}, \alpha_{\mathcal{H}_{\mathrm{j}, \eta}}\right)$ in the same way as $\left(A_{\mathrm{j}, \text { aux }}^{\nabla}, \lambda_{\mathrm{j}, \text { aux }}^{\nabla}, i_{\mathrm{j}, \text { aux }}^{\nabla}, \alpha_{\mathcal{H}_{\mathrm{j}, \text { aux }}}^{\nabla}\right)$ is induced by $\left(A_{\mathrm{j}}, \lambda_{\mathrm{j}}, i_{\mathrm{j}}, \alpha_{\mathcal{H}_{\mathrm{j}}}\right)$ in Proposition 4.12 . Therefore, by the theory of

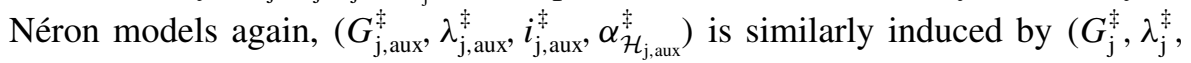
$i_{\mathrm{j}}^{\ddagger}, \alpha_{\mathcal{H}_{\mathrm{j}}}^{\ddagger}$ ). By functoriality of the association of degeneration data, the above object in $\mathrm{DD}_{\mathrm{PEL}, \mathrm{M}_{\mathcal{H}_{\mathrm{j}, \text { aux }}}}(V)$ is also induced by the object in $\operatorname{DD}_{\mathrm{PEL}, \mathrm{M}_{\mathcal{H}_{\mathrm{j}}}}(V)$ determined by $\left(G_{\mathrm{j}}^{\ddagger}, \lambda_{\mathrm{j}}^{\ddagger}, i_{\mathrm{j}}^{\ddagger}, \alpha_{\mathcal{H}_{\mathrm{j}}}^{\ddagger}\right)$ in the statement of the proposition. Hence, up to modifying the choice of the representative $\left(\mathrm{Z}_{\mathcal{H}_{\mathrm{j}, \text { aux }}}^{\ddagger}, \Phi_{\mathcal{H}_{\mathrm{j}, \text { aux }}}^{\ddagger}, \delta_{\mathcal{H}_{\mathrm{j}, \text { aux }}}^{\ddagger}\right)$ above, we may and we shall assume that it is assigned to $\left(\mathrm{Z}_{\mathcal{H}_{\mathrm{j}}}^{\ddagger}, \Phi_{\mathcal{H}_{\mathrm{j}}}^{\ddagger}, \delta_{\mathcal{H}_{\mathrm{j}}}^{\ddagger}\right)$ via (5.8), and that (5.10) maps $v \circ B_{\mathrm{j}}^{\ddagger} \in \mathbf{S}_{\Phi_{\mathcal{H}_{\mathrm{j}}}^{\ddagger}}^{\vee}$ to $v \circ B_{\mathrm{j}, \text { aux }}^{\ddagger} \in \mathbf{S}_{\Phi_{\mathcal{H}_{\mathrm{j}, \text { aux }}^{\ddagger}}^{\vee}}^{\vee}$. 
Thus, for each $\mathrm{j} \in \mathrm{J}$, the representative $\left(\mathrm{Z}_{\mathcal{H}_{\mathrm{j}, \text { aux }}}^{\ddagger}, \Phi_{\mathcal{H}_{\mathrm{j}, \text { aux }}}^{+}, \delta_{\mathcal{H}_{\mathrm{j}, \text { aux }}}^{\ddagger}\right)$ is assigned to $\left(\mathrm{Z}_{\mathcal{H}}^{\ddagger}, \Phi_{\mathcal{H}}^{\ddagger}, \delta_{\mathcal{H}}^{\ddagger}\right)$ via (5.13), and (5.16) maps $v \circ B^{\ddagger} \in \mathbf{S}_{\Phi_{\mathcal{H}}^{\ddagger}}^{\vee}$ to $v \circ B_{\mathrm{j} \text {,aux }}^{\ddagger} \in \mathbf{S}_{\Phi_{\mathcal{H}}^{\ddagger} \text {,aux }}^{\vee}$.

Suppose there exists a morphism $S \rightarrow \overrightarrow{\mathrm{M}}_{\mathcal{H}, \Sigma}^{\text {tor }}$ extending (7.15). For each $\mathrm{j} \in \mathrm{J}$, its composition with the canonical morphism $\overrightarrow{\mathrm{M}}_{\mathcal{H}, \Sigma}^{\text {tor }} \rightarrow \mathrm{M}_{\mathcal{H}_{\mathrm{j}, \text { aux }}, \Sigma_{\mathrm{j}, \text { aux }}}^{\text {tor }}$ induced by (7.8) defines a morphism $S \rightarrow \mathrm{M}_{\mathcal{H}_{\mathrm{j}, \text { aux }}, \Sigma_{\mathrm{j}, \text { aux }}}^{\text {tor }}$ extending (7.16). By [52, IX, 1.4], [10, Ch. I, Proposition 2.7], or [30, Proposition 3.3.1.5], this forces the degenerating family $\left(G_{\mathrm{j}, \text { aux }}^{\dagger}, \lambda_{\mathrm{j} \text {,aux }}^{\dagger}, i_{\mathrm{j} \text {,aux }}^{\dagger}, \alpha_{\mathcal{H}_{\mathrm{j}, \text { aux }}}^{\dagger}\right)$ of type $\mathrm{M}_{\mathcal{H}_{\mathrm{j} \text {,aux }}}$ over $S$ to be isomorphic to the pullback of the tautological degenerating family $\left(G_{\mathrm{j}, \text { aux }}, \lambda_{\mathrm{j} \text {,aux }}\right.$, $\left.i_{\mathrm{j}, \text { aux }}, \alpha_{\mathcal{H}_{\mathrm{j}, \text { aux }}}\right)$ over $\mathrm{M}_{\mathcal{H}_{\mathrm{j}, \text { aux }}, \Sigma_{\mathrm{j}, \text { aux }}}^{\text {tor }}$ By [30, Theorem 6.4.1.1(6)], there is some $\sigma_{\mathrm{j}, \text { aux }}^{\ddagger} \in$ $\Sigma_{\Phi_{\mathcal{H}_{\mathrm{j}, \text { aux }}}^{\ddagger}}$ such that the closure $\bar{\sigma}_{\mathrm{j} \text {,aux }}^{\ddagger}$ of $\sigma_{\mathrm{j} \text {,aux }}^{\ddagger}$ in $\left(\mathbf{S}_{\Phi_{\mathcal{H}_{\mathrm{j}, \text { aux }}}}\right)_{\mathbb{R}}^{\vee}$ contains all $v \circ B_{\mathrm{j} \text {,aux }}^{\ddagger}$. Let $\sigma^{\ddagger} \in \Sigma_{\Phi_{\mathcal{H}}^{\ddagger}}$ be the pullback of $\prod_{\mathrm{j} \in \mathrm{J}} \sigma_{\mathrm{j}, \text { aux }}^{\ddagger}$ under the map (5.20). Then $\bar{\sigma}^{\ddagger}$ contains all $v \circ B^{\ddagger}$ as in the statement of the proposition.

Conversely, suppose that there exist $\sigma^{\ddagger} \in \Sigma_{\Phi_{\mathcal{H}}^{\ddagger}}$ such that $\bar{\sigma}^{\ddagger}$ contains all $v \circ B^{\ddagger}$ as in the statement of the theorem. By definition, there exist $\sigma_{\mathrm{j} \text {, aux }}^{\ddagger} \in \Sigma_{\Phi_{\mathcal{H}_{\mathrm{j}} \text {,aux }}^{\ddagger}}$, for all $\mathrm{j} \in \mathrm{J}$, such that $\sigma^{\ddagger}$ is the pullback of $\prod_{\mathrm{j} \in \mathrm{J}} \sigma_{\mathrm{j} \text {,aux }}^{\ddagger}$ under the map (5.20). Hence, for each $\mathrm{j} \in \mathrm{J}$, all $v \circ B_{\mathrm{j} \text {,aux }}^{\ddagger}$ as above are contained in $\bar{\sigma}_{\mathrm{j}, \text { aux }}^{\ddagger}$, and it follows from [30, Theorem 6.4.1.1(6)] that there is a canonical morphism

$$
S \rightarrow \mathrm{M}_{\mathcal{H}_{\mathrm{j}, \text { aux }}, \Sigma_{\mathrm{j}, \text { aux }}}^{\text {tor }}
$$

under which the degenerating family $\left(G_{\mathrm{j}, \text { aux }}^{\dagger}, \lambda_{\mathrm{j} \text {,aux }}^{\dagger}, i_{\mathrm{j}, \text { aux }}^{\dagger}, \alpha_{\mathcal{H}_{\mathrm{j}, \text { aux }}}^{\dagger}\right)$ is the pullback of the tautological degenerating family $\left(G_{\mathrm{j}, \text { aux }}, \lambda_{\mathrm{j} \text {,aux }}, i_{\mathrm{j}, \text { aux }}, \alpha_{\mathcal{H}_{\mathrm{j}, \text { aux }}}\right)$ over $\mathrm{M}_{\mathcal{H}_{\mathrm{j}, \text { aux }}, \Sigma_{\mathrm{j}, \text { aux }} \text { tor }}$ (although the universal property in [30, Theorem 6.4.1.1(6)] is defined using all morphisms $\operatorname{Spec}(V) \rightarrow S$ centered at a geometric point $\bar{s}$ of $S$, the condition that

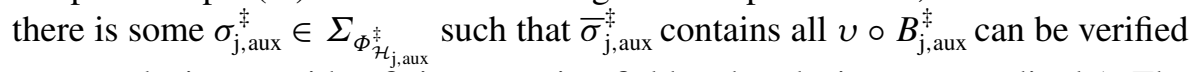
up to replacing $K$ with a finite extension field and replacing $V$ accordingly). The morphisms (7.19), for all $\mathrm{j} \in \mathrm{J}$, induce a canonical morphism

$$
S \rightarrow \prod_{\mathrm{j} \in \mathrm{J}} \mathrm{M}_{\mathcal{H}_{\mathrm{j}, \text { aux },}^{\text {tor }} \Sigma_{\mathrm{j}, \text { aux }}}
$$

Since (7.17) is the composition of (7.15) with (6.2) by assumption, the morphism (7.20) induced the desired morphism (7.18) by the definition of $\overrightarrow{\mathrm{M}}_{\mathcal{H}, \Sigma}^{\text {tor }}$ as the normalization of $\prod_{\mathrm{j} \in \mathrm{J}} \mathrm{M}_{\mathcal{H}_{\mathrm{j}, \text { aux }}, \Sigma_{\mathrm{j}, \text { aux }}}^{\text {tor }} \mathrm{M}_{\mathcal{H}}$ under the morphism (7.7), as desired. 


\section{Putative boundary charts}

The goal of this section is to construct the schemes $\overrightarrow{\mathrm{M}}_{\mathcal{H}}^{\Phi_{\mathcal{H}}}, \vec{C}_{\Phi_{\mathcal{H}}, \delta_{\mathcal{H}}}, \vec{\Xi}_{\Phi_{\mathcal{H}}, \delta_{\mathcal{H}}}$, $\vec{\Xi}_{\Phi_{\mathcal{H}}, \delta_{\mathcal{H}}}(\sigma)$, and $\vec{\Xi}_{\Phi_{\mathcal{H}}, \delta_{\mathcal{H}}, \sigma}$, and the formal scheme $\overrightarrow{\mathfrak{X}}_{\Phi_{\mathcal{H}}, \delta_{\mathcal{H}}, \sigma}$ over $\overrightarrow{\mathrm{M}}_{\mathcal{H}}^{\mathrm{Z}_{\mathcal{H}}}$ (whose meanings will be explained below), which will be useful for defining a locally closed subscheme $\vec{Z}_{\left[\left(\Phi_{\mathcal{H}}, \delta_{\mathcal{H}}, \sigma\right)\right]}$ of $\overrightarrow{\mathrm{M}}_{\mathcal{H}, \Sigma}^{\text {tor }}$, and for describing the formal completion of $\overrightarrow{\mathrm{M}}_{\mathcal{H}, \Sigma}^{\text {tor }}$ along $\vec{Z}_{\left[\left(\Phi_{\mathcal{H}}, \delta_{\mathcal{H}}, \sigma\right)\right]}$, in the next two sections.

Proposition 8.1. Let $\overrightarrow{\mathrm{M}}_{\mathcal{H}}^{\mathrm{Z}_{\mathcal{H}}}$ be as in Proposition 7.4. Let $\overrightarrow{\mathrm{M}}_{\mathcal{H}}^{\Phi_{\mathcal{H}}}$ denote the normalization of $\prod_{\mathrm{j} \in \mathrm{J}} \mathrm{M}_{\mathcal{H}_{\mathrm{j} \text {,aux }}}^{\Phi_{\mathcal{H} \text {,aux }}}$ in $\mathrm{M}_{\mathcal{H}}^{\Phi_{\mathcal{H}}}$ under the morphism

$$
\mathrm{M}_{\mathcal{H}}^{\Phi_{\mathcal{H}}} \rightarrow \prod_{\mathrm{j} \in \mathrm{J}} \mathrm{M}_{\mathcal{H}_{\mathrm{j}, \text { aux }}}^{\Phi_{\mathcal{H}_{\mathrm{j}} \text { aux }}}
$$

defined by comparing the universal properties of $\mathrm{M}_{\mathcal{H}}^{\Phi_{\mathcal{H}}} \cong \mathrm{M}_{\mathcal{H}_{\mathrm{j}}}^{\Phi_{\mathcal{H}_{\mathrm{j}}}}$ (see (3.6)) and $\mathrm{M}_{\mathcal{H}_{\mathrm{j}, \text { aux }}}^{\Phi_{\mathcal{H}_{\mathrm{a}, \text { ax }}}}$, for all $\mathrm{j} \in \mathrm{J}$ (see [30, Definition 5.4.2.6 and the errata]; cf. [31, Lemma 1.3.2.5 and the paragraph preceding it]). Then the morphism (8.2) induces a finite morphism

$$
\overrightarrow{\mathrm{M}}_{\mathcal{H}}^{\Phi_{\mathcal{H}}} \rightarrow \prod_{\mathrm{j} \in \mathrm{J}} \mathrm{M}_{\mathcal{H}_{\mathrm{j}, \text { aux }}}^{\Phi_{\mathcal{H}, \text { aux }}}
$$

compatible with (7.6), and the canonical morphism $\overrightarrow{\mathrm{M}}_{\mathcal{H}}^{\Phi_{\mathcal{H}}} \rightarrow \overrightarrow{\mathrm{M}}_{\mathcal{H}}^{Z_{\mathcal{H}}}$ extending the canonical finite étale morphism $\mathrm{M}_{\mathcal{H}}^{\Phi_{\mathcal{H}}} \rightarrow \mathrm{M}_{\mathcal{H}}^{Z_{\mathcal{H}}}$ is also finite.

Proof. The canonical morphism $\overrightarrow{\mathrm{M}}_{\mathcal{H}}^{\Phi_{\mathcal{H}}} \rightarrow \overrightarrow{\mathrm{M}}_{\mathcal{H}}^{\mathcal{Z}_{\mathcal{H}}}$ is finite because so are the

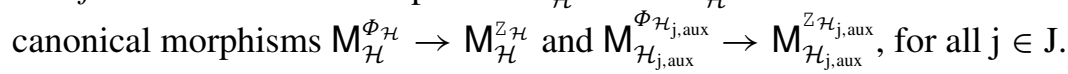

PROPOSITION 8.4. Let $\vec{C}_{\Phi_{\mathcal{H}}, \delta_{\mathcal{H}}}$ denote the normalization of $\prod_{\mathrm{j} \in \mathrm{J}} C_{\Phi_{\mathcal{H}_{\mathrm{j}, \text { aux }}}, \delta_{\mathcal{H}_{\mathrm{j}} \text {,aux }}}$ in $C_{\Phi_{\mathcal{H}}, \delta_{\mathcal{H}}}$ under the morphism

$$
C_{\Phi_{\mathcal{H}}, \delta \mathcal{H}} \rightarrow \prod_{\mathrm{j} \in \mathrm{J}} C_{\Phi_{\mathcal{H}_{\mathrm{j}, \text { aux }}, \delta \mathcal{H}_{\mathrm{j}, \text { aux }}}}
$$

defined by comparing the universal properties of $C_{\Phi_{\mathcal{H}}, \delta_{\mathcal{H}}} \cong C_{\Phi_{\mathcal{H}_{\mathrm{j}}}, \delta_{\mathcal{H}_{\mathrm{j}}}}$ (see (3.14)) and $C_{\Phi_{\mathcal{H}_{\mathrm{j}, \text { aux }}}, \delta_{\mathcal{H}_{\mathrm{j}, \text { aux }}}}$ for all $\mathrm{j} \in \mathrm{J}$ (parameterizing the additional objects $\left(c_{\mathcal{H}_{\mathrm{j}}}\right.$,

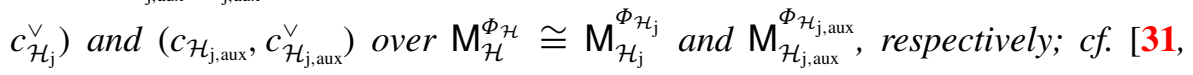


Lemma 1.3.2.11 and Proposition 1.3.2.12]). Then the morphism (8.5) induces a finite morphism

$$
\vec{C}_{\Phi_{\mathcal{H}}, \delta \mathcal{H}} \rightarrow \prod_{\mathrm{j} \in \mathrm{J}} C_{\Phi_{\mathcal{H}_{\mathrm{j}, \text { aux }}, \delta_{\mathcal{H}}, \mathrm{aux}}}
$$

compatible with (8.3), and the canonical morphism $\vec{C}_{\Phi_{\mathcal{H}}, \delta_{\mathcal{H}}} \rightarrow \overrightarrow{\mathrm{M}}_{\mathcal{H}}^{\Phi_{\mathcal{H}}}$ extending the canonical abelian scheme torsor $C_{\Phi_{\mathcal{H}}, \delta_{\mathcal{H}}} \rightarrow \mathrm{M}_{\mathcal{H}}^{\Phi_{\mathcal{H}}}$ is proper.

Proof. The canonical morphism $\vec{C}_{\Phi_{\mathcal{H}}, \delta_{\mathcal{H}}} \rightarrow \overrightarrow{\mathrm{M}}_{\mathcal{H}}^{\Phi_{\mathcal{H}}}$ is proper because so are the

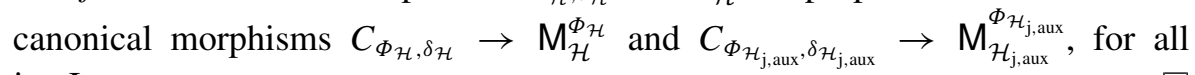
$\mathrm{j} \in \mathrm{J}$.

PROPOSITION 8.7. Let $\vec{\Xi}_{\Phi_{\mathcal{H}}, \delta_{\mathcal{H}}}$ denote the normalization of $\prod_{\mathrm{j} \in \mathrm{J}} \Xi_{\Phi_{\mathcal{H}_{\mathrm{j}, \text { aux }}, \delta \mathcal{H}_{\mathrm{j}, \text { aux }}}}$ in $\Xi_{\Phi_{\mathcal{H}}, \delta_{\mathcal{H}}}$ under the morphism

$$
\Xi_{\Phi_{\mathcal{H}}, \delta_{\mathcal{H}}} \rightarrow \prod_{\mathrm{j} \in \mathrm{J}} \Xi_{\Phi_{\mathcal{H}_{\mathrm{j}, \text { aux }},}, \mathcal{S}_{\mathcal{H}_{\mathrm{j}, \text { aux }}}}
$$

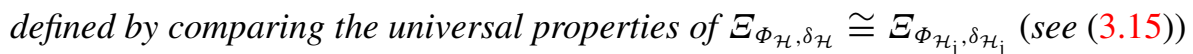

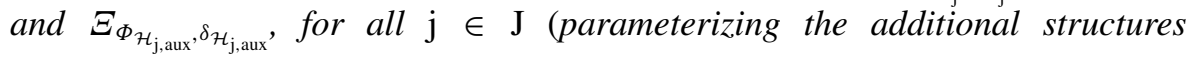

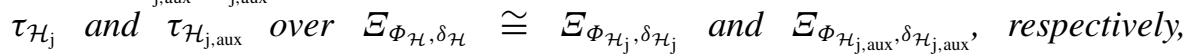
without their respective positive conditions; cf. [31, Lemma 1.3.2.28 and Proposition 1.3.2.31]). Then the morphism (8.8) induces a finite morphism

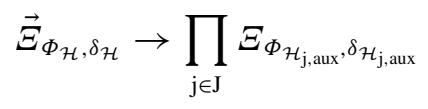

compatible with (8.6), and the canonical morphism $\vec{\Xi}_{\Phi_{\mathcal{H}}, \delta_{\mathcal{H}}} \rightarrow \vec{C}_{\Phi_{\mathcal{H}}, \delta_{\mathcal{H}}}$ extending the canonical morphism $\Xi_{\Phi_{\mathcal{H}}, \delta_{\mathcal{H}}} \rightarrow C_{\Phi_{\mathcal{H}}, \delta_{\mathcal{H}}}$ also admits a canonical extension of the $E_{\Phi_{\mathcal{H}}}$-torsor structure of the latter (see [30, Theorem 6.4.1.1(5)]), where $E_{\Phi_{\mathcal{H}}}$ is the split torus with character group $\mathbf{S}_{\Phi_{\mathcal{H}}}$.

The $E_{\Phi_{\mathcal{H}}}$-torsor structure of ${\stackrel{\Xi}{\Phi_{\mathcal{H}}, \delta_{\mathcal{H}}}}$ defines a canonical homomorphism

$$
\mathbf{S}_{\Phi_{\mathcal{H}}} \rightarrow \underline{\operatorname{Pic}}\left(\vec{C}_{\Phi_{\mathcal{H}}, \delta_{\mathcal{H}}}\right): \ell \mapsto \vec{\Psi}_{\Phi_{\mathcal{H}}, \delta_{\mathcal{H}}}(\ell),
$$

giving for each $\ell \in \mathbf{S}_{\Phi_{\mathcal{H}}}$ an invertible sheaf $\vec{\Psi}_{\Phi_{\mathcal{H}}, \delta_{\mathcal{H}}}(\ell)$ over $\vec{C}_{\Phi_{\mathcal{H}}, \delta_{\mathcal{H}}}$ (up to isomorphism), together with isomorphisms

$$
\vec{\Delta}_{\Phi_{\mathcal{H}}, \delta_{\mathcal{H}}, \ell, \ell^{\prime}}^{*}: \vec{\Psi}_{\Phi_{\mathcal{H}}, \delta_{\mathcal{H}}}(\ell) \underset{\mathscr{O}_{\bar{C}_{\Phi_{\mathcal{H}}, \delta_{\mathcal{H}}}}}{\otimes} \vec{\Psi}_{\Phi_{\mathcal{H}}, \delta_{\mathcal{H}}}\left(\ell^{\prime}\right) \stackrel{\sim}{\rightarrow} \vec{\Psi}_{\Phi_{\mathcal{H}}, \delta_{\mathcal{H}}}\left(\ell+\ell^{\prime}\right)
$$


for all $\ell, \ell^{\prime} \in \mathbf{S}_{\Phi_{\mathcal{H}}}$, satisfying the necessary compatibilities with each other making $\bigoplus_{\ell \in \mathbf{S}_{\Phi_{\mathcal{H}}}} \vec{\Psi}_{\Phi_{\mathcal{H}}, \delta_{\mathcal{H}}}(\ell)$ an $\mathscr{O}_{\vec{C}_{\Phi_{\mathcal{H}},}, \mathcal{H}_{\mathcal{H}}}$-algebra, such that

$$
\vec{\Xi}_{\Phi_{\mathcal{H}}, \delta_{\mathcal{H}}} \cong \operatorname{Spec}_{\mathscr{C}_{\vec{C}_{\Phi_{\mathcal{H}}, \delta \mathcal{H}}}}\left(\bigoplus_{\ell \in \mathbf{S}_{\Phi_{\mathcal{H}}}} \vec{\Psi}_{\Phi_{\mathcal{H}}, \delta_{\mathcal{H}}}(\ell)\right)
$$

(cf. [30, Proposition 6.2.4.7 and (6.2.4.8); see also the errata]).

The canonical morphism

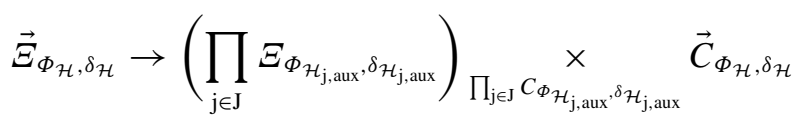

induced by (8.6) and (8.9) is equivariant with the finite homomorphism

$$
E_{\Phi_{\mathcal{H}}} \rightarrow \prod_{\mathrm{j} \in \mathrm{J}} E_{\Phi_{\mathcal{H}_{\mathrm{j}, \text { aux }}}}
$$

dual to (5.22), which is finite because (5.21) is surjective. If (5.22) maps $\left(\ell_{\mathrm{j}, \text { aux }}\right)_{\mathrm{j} \in \mathrm{J}} \in \prod_{\mathrm{j} \in \mathrm{J}} \mathbf{S}_{\Phi_{\mathcal{H}_{\mathrm{j}, \text { aux }}}}$ to $\ell \in \mathbf{S}_{\Phi_{\mathcal{H}}}$, then $\vec{\Psi}_{\Phi_{\mathcal{H}}, \delta_{\mathcal{H}}}(\ell)$ is isomorphic to the pullback of $\bigotimes_{\mathrm{j} \in \mathrm{J}} \Psi_{\Phi_{\mathcal{H}_{\mathrm{j}, \text { aux }}}, \delta \mathcal{H}_{\mathrm{j}, \text { aux }}}\left(\ell_{\mathrm{j}, \text { aux }}\right)$ under (8.6).

Proof. By the universal properties of $\Xi_{\Phi_{\mathcal{H}}, \delta_{\mathcal{H}}}$ and $\Xi_{\Phi_{\mathcal{H}_{\mathrm{j}, \text { aux }}, \delta_{\mathcal{H}} \text {,aux }}}$, for all $\mathrm{j} \in \mathrm{J}$, the canonical morphism (8.8) is equivariant with the finite homomorphism (8.13). Suppose that the $E_{\Phi_{\mathcal{H}}}$-torsor $\Xi_{\Phi_{\mathcal{H}}, \delta_{\mathcal{H}}} \rightarrow C_{\Phi_{\mathcal{H}}, \delta_{\mathcal{H}}}$ extends to some $E_{\Phi_{\mathcal{H}}}$-torsor

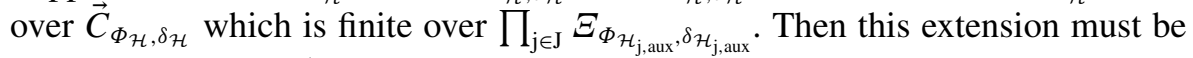
isomorphic to the $\vec{\Xi}_{\Phi_{\mathcal{H}}, \delta_{\mathcal{H}}}$ defined by normalization as in the statement of the proposition, and the canonically induced morphism (8.12) must be equivariant with the homomorphism (8.13). As usual, for each $\ell \in \mathbf{S}_{\Phi_{\mathcal{H}}}$, we define $\vec{\Psi}_{\Phi_{\mathcal{H}}, \delta_{\mathcal{H}}}(\ell)$ to be the subsheaf of $\left(\vec{\Xi}_{\Phi_{\mathcal{H}}, \delta_{\mathcal{H}}} \rightarrow \vec{C}_{\Phi_{\mathcal{H}}, \delta_{\mathcal{H}}}\right)_{*} \mathscr{O}_{\vec{\Xi}_{\Phi_{\mathcal{H}}, \delta_{\mathcal{H}}}}$ on which $E_{\Phi_{\mathcal{H}}}$ acts by the character $\ell$; for $\ell, \ell^{\prime} \in \mathbf{S}_{\Phi_{\mathcal{H}}}$, we define $\vec{\Delta}_{\Phi_{\mathcal{H}}, \delta_{\mathcal{H}}, \ell, \ell^{\prime}}^{*}$ to be the isomorphism induced by the $\mathscr{O}_{\vec{C}_{\Phi_{\mathcal{H}}, \delta_{\mathcal{H}}}}$-algebra structure of $\left(\vec{\Xi}_{\Phi_{\mathcal{H}}, \delta_{\mathcal{H}}} \rightarrow \vec{C}_{\Phi_{\mathcal{H}}, \delta_{\mathcal{H}}}\right)_{*} \mathscr{O}_{\vec{\Xi}_{\Phi_{\mathcal{H}}, \delta_{\mathcal{H}}}}$. Then the remaining assertions of the proposition follow from the constructions.

It remains to show that the $E_{\Phi_{\mathcal{H}}}$-torsor $\Xi_{\Phi_{\mathcal{H}}, \delta_{\mathcal{H}}} \rightarrow C_{\Phi_{\mathcal{H}}, \delta_{\mathcal{H}}}$ extends to some $E_{\Phi_{\mathcal{H}}}$-torsor over $\vec{C}_{\Phi_{\mathcal{H}}, \delta_{\mathcal{H}}}$ that is finite over $\prod_{\mathrm{j} \in \mathrm{J}} \Xi_{\Phi_{\mathcal{H}_{\mathrm{j}, \text { aux }}}, \delta_{\mathcal{H}_{\mathrm{j}, \text { aux }}}}$, which we shall abusively denote by $\vec{\Xi}_{\Phi_{\mathcal{H}}, \delta \mathcal{H}}$. Take any $\mathrm{j}_{0} \in \mathrm{J}$, and take any integer $n \geqslant 1$ such that $\mathcal{U}_{\mathrm{j}_{0}}(n):=\operatorname{ker}\left(\mathrm{G}_{\mathrm{j}_{0}}(\hat{\mathbb{Z}}) \rightarrow \mathrm{G}_{\mathrm{j}_{0}}(\hat{\mathbb{Z}} / n \hat{\mathbb{Z}})=\mathrm{G}_{\mathrm{j}_{0}}(\mathbb{Z} / n \mathbb{Z})\right) \subset \mathcal{H}_{\mathrm{j}_{0}}$. Let $\mathcal{H}^{\prime}$ be the pullback of $\mathcal{U}_{\mathrm{j}_{0}}(n)$ under the canonical homomorphism $\mathrm{G}\left(\mathbb{A}^{\infty}\right) \cong \mathrm{G}_{\mathrm{j}_{0}}\left(\mathbb{A}^{\infty}\right)$, and let $\left(\Phi_{\mathcal{H}^{\prime}}, \delta_{\mathcal{H}^{\prime}}\right)$ be any cusp label for $\mathrm{M}_{\mathcal{H}^{\prime}}$ lifting $\left(\Phi_{\mathcal{H}}, \delta_{\mathcal{H}}\right)$. By the construction in [30, Section 6.2.4 and the errata], and by Zariski's main theorem (see [14, III-1, 4.4.3, 4.4.11]), we can construct $\vec{\Xi}_{\Phi_{\mathcal{H}}, \delta_{\mathcal{H}}} \rightarrow \vec{C}_{\Phi_{\mathcal{H}}, \delta_{\mathcal{H}}}$ as an equivariant 
quotient of $\vec{\Xi}_{\Phi_{\mathcal{H}^{\prime}}, \delta_{\mathcal{H}^{\prime}}} \rightarrow \vec{C}_{\Phi_{\mathcal{H}^{\prime}}, \delta_{\mathcal{H}^{\prime}}}$, as soon as the latter is known. Hence, we may replace $\mathcal{H}$ with $\mathcal{H}^{\prime}$. Since $\vec{C}_{\Phi_{\mathcal{H}}, \delta_{\mathcal{H}}}$ can be alternatively constructed by normalization over some product of naive moduli (cf. the proof of Proposition 6.1 and the construction of $\dddot{C}_{\Phi_{n}}$ in [30, Section 6.2.3]), we may assume that the tautological structure $\left(c_{\mathrm{j}_{0}, n}: \frac{1}{n} X_{\mathrm{j}_{0}} \rightarrow B_{\mathrm{j}_{0}}^{\vee}, c_{\mathrm{j}_{0}, n}^{\vee}: \frac{1}{n} Y_{\mathrm{j}_{0}} \rightarrow B_{\mathrm{j}_{0}}\right)$ over $C_{\Phi_{\mathcal{H}}, \delta_{\mathcal{H}}}$ extends to some $\left(\vec{c}_{\mathrm{j}_{0}, n}: \frac{1}{n} X_{\mathrm{j}_{0}} \rightarrow \vec{B}_{\mathrm{j}_{0}}^{\vee}, \vec{c}_{\mathrm{j}_{0}, n}^{\vee}: \frac{1}{n} Y_{\mathrm{j}_{0}} \rightarrow \vec{B}_{\mathrm{j}_{0}}\right.$ ) over $\vec{C}_{\Phi_{\mathcal{H}}, \delta_{\mathcal{H}}}$ (cf. Proposition 7.4). By the same construction of $\dddot{\Xi}_{\Phi_{n}}$ as in [30, Section 6.2.3], the naive structures $\vec{\tau}_{\mathrm{j}_{0}, n}$ (without pairing and liftability conditions) are parameterized by some $\dddot{\Xi} \rightarrow \vec{C}_{\Phi_{\mathcal{H}}, \delta_{\mathcal{H}}}$ which factors as the composition of a $E_{\Phi_{\mathcal{H}}}$-torsor $\dddot{\Xi} \rightarrow \dddot{\Xi}^{\prime}$ and a torsor $\dddot{\Xi}^{\prime} \rightarrow \vec{C}_{\Phi_{\mathcal{H}}, \delta_{\mathcal{H}}}$ under a finite group $\dddot{E}^{\prime}$ of multiplicative type. By comparing universal properties, there is a canonical $E_{\Phi_{\mathcal{H}}}$-equivariant morphism $\Xi_{\Phi_{\mathcal{H}}, \delta_{\mathcal{H}}} \rightarrow \dddot{\Xi}$, which induces a morphism $C_{\Phi_{\mathcal{H}}, \delta_{\mathcal{H}}} \rightarrow \dddot{\Xi}^{\prime}$. On the other hand, up to replacing the groups $\mathcal{H}_{\mathrm{j} \text {,aux }}$ with finite index principal level subgroups, for all $\mathrm{j} \in \mathrm{J}$, and replacing $n$ with a multiple if necessary, the pullback $\Xi_{\text {aux }}$ of $\prod_{\mathrm{j} \in \mathrm{J}} \Xi_{\Phi_{\mathcal{H}_{\mathrm{j}, \text { aux }}}, \delta_{\mathcal{H}_{\mathrm{j}, \text { aux }}}}$ to $\vec{C}_{\Phi_{\mathcal{H}}, \delta_{\mathcal{H}}}$ can be embedded in some similar composition $\dddot{\Xi}_{\text {aux }} \rightarrow \dddot{\Xi}_{\text {aux }}^{\prime} \rightarrow \vec{C}_{\Phi_{\mathcal{H}}, \delta_{\mathcal{H}}}$ of torsors under $\prod_{\mathrm{j} \in \mathrm{J}} E_{\Phi_{\mathcal{H}_{\mathrm{j} \text {,aux }}}}$ and a finite group of multiplicative type, respectively, together with a finite morphism $\dddot{\Xi} \rightarrow \dddot{\Xi}_{\text {aux }}$ equivariant with the finite homomorphism (8.13), whose precomposition with the above morphism $\Xi_{\Phi_{\mathcal{H}}, \delta_{\mathcal{H}}} \rightarrow \dddot{\Xi}$ lands in $\Xi_{\text {aux. }}$. Since $\vec{C}_{\Phi_{\mathcal{H}}, \delta_{\mathcal{H}}}$ is noetherian and normal, the above morphism $C_{\Phi_{\mathcal{H}}, \delta_{\mathcal{H}}} \rightarrow \dddot{\Xi}^{\prime}$ extends to a section $\vec{C}_{\Phi_{\mathcal{H}}, \delta_{\mathcal{H}}} \rightarrow \dddot{\Xi}^{\prime}$ of the above morphism $\dddot{\Xi}^{\prime} \rightarrow \vec{C}_{\Phi_{\mathcal{H}}, \delta_{\mathcal{H}}}$, under which the pullback of the $E_{\Phi_{\mathcal{H}}}$ torsor $\dddot{\Xi} \rightarrow \dddot{\Xi}^{\prime}$ defines an $E_{\Phi_{\mathcal{H}}}$-torsor extension $\vec{\Xi}_{\Phi_{\mathcal{H}}, \delta_{\mathcal{H}}}$ over $\vec{C}_{\Phi_{\mathcal{H}}, \delta_{\mathcal{H}}}$, with a finite morphism to $\prod_{\mathrm{j} \in \mathrm{J}} \Xi_{\Phi_{\mathcal{H}_{\mathrm{j}, \mathrm{aux}}}, \delta_{\mathcal{H}_{\mathrm{j}, \mathrm{aux}}}}$, as desired.

Proposition 8.14. Suppose that $\sigma_{\mathrm{j}, \text { aux }} \in \Sigma_{\Phi_{\mathcal{H}_{\mathrm{j}, \text { aux }}}}$ for each $\mathrm{j} \in \mathrm{J}$, and that $\sigma \in \Sigma_{\Phi_{\mathcal{H}}}$ is the pullback of $\prod_{\mathrm{j} \in \mathrm{J}} \sigma_{\mathrm{j} \text {,aux }}$ under the map (5.20). Consider the affine toroidal embedding as in (3.17), which extends to the affine toroidal embedding

$$
\vec{\Xi}_{\Phi_{\mathcal{H}}, \delta \mathcal{H}} \hookrightarrow \vec{\Xi}_{\Phi_{\mathcal{H}}, \delta \mathcal{H}}(\sigma):=\underline{\operatorname{Spec}}_{\mathscr{O}_{\vec{C}_{\Phi_{\mathcal{H}}, \delta \mathcal{H}}}}\left(\bigoplus_{\ell \in \sigma^{\vee}} \vec{\Psi}_{\Phi_{\mathcal{H}}, \delta \mathcal{H}}(\ell)\right)
$$

over $\vec{C}_{\Phi_{\mathcal{H}}, \delta \mathcal{H}}$, where the invertible sheaves $\vec{\Psi}_{\Phi_{\mathcal{H}}, \delta \mathcal{H}}(\ell)$ are as in [30, Proposition 6.2.4.7 and (6.2.4.8); see also the errata] as well. Then the canonical morphism

$$
\vec{\Xi}_{\Phi_{\mathcal{H}}, \delta_{\mathcal{H}}}(\sigma) \rightarrow \vec{C}_{\Phi_{\mathcal{H}}, \delta_{\mathcal{H}}}
$$

is faithfully flat and has geometrically normal and Cohen-Macaulay fibers, and $\vec{\Xi}_{\Phi_{\mathcal{H}}, \delta_{\mathcal{H}}}$ is fiberwise dense in $\vec{\Xi}_{\Phi_{\mathcal{H}}, \delta_{\mathcal{H}}}(\sigma)$. If $\sigma$ is smooth, then (8.16) is smooth and surjective. Moreover, whether $\sigma$ is smooth or not, $\vec{\Xi}_{\Phi_{\mathcal{H}}, \delta_{\mathcal{H}}}(\sigma)$ is normal 
and is canonically isomorphic to the normalization of $\prod_{\mathrm{j} \in \mathrm{J}} \Xi_{\Phi_{\mathcal{H}_{\mathrm{j}, \text { aux }}}, \delta_{\mathcal{H}_{\mathrm{j}} \text {,aux }}}\left(\sigma_{\mathrm{j} \text {,aux }}\right)$ in $\Xi_{\Phi_{\mathcal{H}}, \delta_{\mathcal{H}}}$ under the composition

$$
\Xi_{\Phi_{\mathcal{H}}, \delta_{\mathcal{H}}} \stackrel{(8.8)}{\rightarrow} \prod_{\mathrm{j} \in \mathrm{J}} \Xi_{\Phi_{\mathcal{H}_{\mathrm{j}, \text { aux }}}, \delta_{\mathcal{H}_{\mathrm{j}, \text { aux }}}} \stackrel{\text { can. }}{\rightarrow} \prod_{\mathrm{j} \in \mathrm{J}} \Xi_{\Phi_{\mathcal{H}_{\mathrm{j}, \text { aux }}}, \delta_{\mathcal{H}_{\mathrm{j}, \text { aux }}}}\left(\sigma_{\mathrm{j}, \text { aux }}\right),
$$

which (necessarily uniquely) extends to a finite morphism

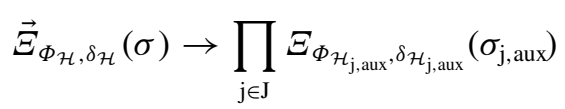

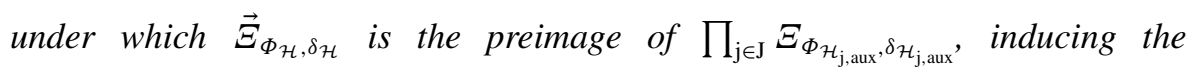
same (8.9).

Proof. Over open subsets of $\vec{C}_{\Phi_{\mathcal{H}}, \delta_{\mathcal{H}}}$ over which the invertible sheaves $\vec{\Psi}_{\Phi_{\mathcal{H}}, \delta_{\mathcal{H}}}(\ell)$ are free for all $\ell \in \mathbf{S}_{\Phi_{\mathcal{H}}}$ (which is possible because $\mathbf{S}_{\Phi_{\mathcal{H}}}$ is finitely generated), the global sections of the $\mathscr{O}_{\vec{C}_{\Phi_{\mathcal{H}}, \delta \mathcal{H}}}$-algebra $\bigoplus_{\ell \in \mathbf{S}_{\Phi_{\mathcal{H}}}} \vec{\Psi}_{\Phi_{\mathcal{H}}, \delta_{\mathcal{H}}}(\ell)$ is the localization of the global sections of the $\mathscr{O}_{\vec{C}_{\Phi_{\mathcal{H}}, \delta_{\mathcal{H}}}}$-algebra $\bigoplus_{\ell \in \sigma^{\vee}} \vec{\Psi}_{\Phi_{\mathcal{H}}, \delta_{\mathcal{H}}}(\ell)$ at a multiplicative subset generated by finitely many elements (which is possible because $\sigma^{\vee}$ is finitely generated; cf. [24, Ch. I, Section 1, Lemma 2]). Hence the morphism (8.15) is an open immersion. The canonical morphism (8.16) is flat because the $\mathscr{O}_{\vec{C}_{\Phi_{\mathcal{H}}, \delta_{\mathcal{H}}}}$-algebra $\bigoplus_{\ell \in \sigma^{\vee}} \vec{\Psi}_{\Phi_{\mathcal{H}}, \delta_{\mathcal{H}}}(\ell)$ is a direct sum of invertible sheaves over $\vec{C}_{\Phi_{\mathcal{H}}, \delta_{\mathcal{H}}}$. By [24, Ch. I, Section 1, Theorem $1^{\prime}$ and 2], the fibers of (8.16) are geometrically normal, and contain the corresponding fibers of $\vec{\Xi}_{\Phi_{\mathcal{H}}, \delta_{\mathcal{H}}}$ as nonempty open dense subsets. By [22, Theorem 1] (cf. [23, Theorem 4.1]), the fibers of (8.16) are Cohen-Macaulay. If $\sigma$ is smooth, then (8.16) is smooth because it is flat and has geometrically regular fibers, by [24, Ch. I, Section 1, Theorem 4]. Whether $\sigma$ is smooth or not, since (8.16) is faithfully flat and of finite type, and since $\vec{C}_{\Phi_{\mathcal{H}}, \delta_{\mathcal{H}}}$ is noetherian and normal, it follows from the normality of the fibers of (8.16) and from [35, 21.E] that $\vec{\Xi}_{\Phi_{\mathcal{H}}, \delta_{\mathcal{H}}}(\sigma)$ is also normal.

By the definition of $\vec{\Xi}_{\Phi_{\mathcal{H}}, \delta_{\mathcal{H}}}$ (see Proposition 8.7), the normalization of $\prod_{\mathrm{j} \in \mathrm{J}} \Xi_{\Phi_{\mathcal{H}_{\mathrm{j}, \text { aux }}}, \delta_{\mathcal{H}_{\mathrm{j}} \text {,aux }}}\left(\sigma_{\mathrm{j} \text {,aux }}\right)$ in $\Xi_{\Phi_{\mathcal{H}}, \delta_{\mathcal{H}}}$ under the composition (8.17) can be identified with the normalization of $\prod_{\mathrm{j} \in \mathrm{J}} \Xi_{\Phi_{\mathcal{H}_{\mathrm{j}, \text { aux }}}, \delta_{\mathcal{H}_{\mathrm{j}} \text {,aux }}}\left(\sigma_{\mathrm{j}, \text { aux }}\right)$ in $\vec{\Xi}_{\Phi_{\mathcal{H}}, \delta_{\mathcal{H}}}$ under the composition

$$
\vec{\Xi}_{\Phi_{\mathcal{H}}, \delta_{\mathcal{H}}} \stackrel{(8.9)}{\rightarrow} \prod_{\mathrm{j} \in \mathrm{J}} \Xi_{\Phi_{\mathcal{H}_{\mathrm{j}, \text { aux }}}, \delta_{\mathcal{H}_{\mathrm{j}, \text { aux }}}} \stackrel{\text { can. }}{\rightarrow} \prod_{\mathrm{j} \in \mathrm{J}} \Xi_{\Phi_{\mathcal{H}_{\mathrm{j}, \text { aux }}}, \delta_{\mathcal{H}_{\mathrm{j}, \text { aux }}}}\left(\sigma_{\mathrm{j}, \text { aux }}\right) .
$$

Thus it suffices to show that $\vec{\Xi}_{\Phi_{\mathcal{H}}, \delta_{\mathcal{H}}}(\sigma)$ is canonically isomorphic to this normalization, or that (8.19) extends to a finite morphism as in (8.18). Since the 
$\mathbb{Q}_{>0}$-span of $\sigma^{\vee}$ is the image of the $\mathbb{Q}_{>0}$-span of $\prod_{\mathrm{j} \in \mathrm{J}} \sigma_{\mathrm{j} \text {,aux }}^{\vee}$ under the map (5.21) (see [18, Lemma 3.2]), this follows from the last paragraph of Proposition 8.7, as desired.

Let $\sigma$ and $\left\{\sigma_{\mathrm{j}, \text { aux }}\right\}_{\mathrm{j} \in \mathrm{J}}$ be as in Proposition 8.14. Let

$$
\sigma_{0}^{\vee}:=\left\{\ell \in \mathbf{S}_{\Phi_{\mathcal{H}}}:\langle\ell, y\rangle>0 \forall y \in \sigma\right\}
$$

and

$$
\sigma^{\perp}:=\left\{\ell \in \mathbf{S}_{\Phi_{\mathcal{H}}}:\langle\ell, y\rangle=0 \forall y \in \sigma\right\} \cong \sigma^{\vee} / \sigma_{0}^{\vee}
$$

as usual (see [30, Definitions 6.1.1.8 and 6.1.2.5]). Consider the formal completion $\overrightarrow{\mathfrak{X}}_{\Phi_{\mathcal{H}}, \delta_{\mathcal{H}}, \sigma}$ of $\vec{\Xi}_{\Phi_{\mathcal{H}}, \delta \mathcal{H}}(\sigma)$ along its closed subscheme

$$
\vec{\Xi}_{\Phi_{\mathcal{H}}, \delta \mathcal{H}, \sigma}:=\underline{\operatorname{Spec}}_{\mathscr{O}_{\vec{C}_{\Phi_{\mathcal{H}}, \delta_{\mathcal{H}}}}}\left(\bigoplus_{\ell \in \sigma^{\perp}} \vec{\Psi}_{\Phi_{\mathcal{H}}, \delta \mathcal{H}}(\ell)\right),
$$

which extends the formal completion $\mathfrak{X}_{\Phi_{\mathcal{H}}, \delta_{\mathcal{H}}, \sigma}$ of $\Xi_{\Phi_{\mathcal{H}}, \delta_{\mathcal{H}}}(\sigma)$ along its closed subscheme $\Xi_{\Phi_{\mathcal{H}}, \delta_{\mathcal{H}}, \sigma}=$ Spec $_{\mathscr{G}_{C_{\Phi_{\mathcal{H}}, \delta_{\mathcal{H}}}}}\left(\bigoplus_{\ell \in \sigma^{\perp}} \Psi_{\Phi_{\mathcal{H}}, \delta_{\mathcal{H}}}(\ell)\right)$; and consider the

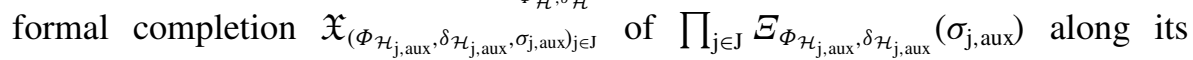

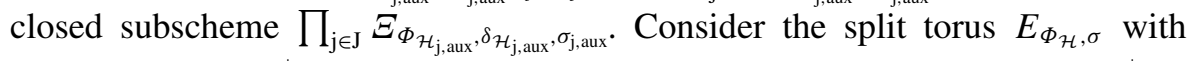
character group $\sigma^{\perp}$, which is the quotient of $E_{\Phi_{\mathcal{H}}}$ dual to the subgroup $\sigma^{\perp}$ of $\mathbf{S}_{\Phi_{\mathcal{H}}}$, as in [30, Theorem 7.2.4.1(5)]. By construction, the canonical morphisms $\Xi_{\Phi_{\mathcal{H}}, \delta_{\mathcal{H}}, \sigma} \rightarrow C_{\Phi_{\mathcal{H}}, \delta_{\mathcal{H}}}$ and $\vec{\Xi}_{\Phi_{\mathcal{H}}, \delta_{\mathcal{H}}, \sigma} \rightarrow \vec{C}_{\Phi_{\mathcal{H}}, \delta_{\mathcal{H}}}$ are (compatible) $E_{\Phi_{\mathcal{H}}, \sigma}$-torsors.

LEMMA 8.20. The subscheme $\vec{\Xi}_{\Phi_{\mathcal{H}}, \delta_{\mathcal{H}}, \sigma}$ of $\vec{\Xi}_{\Phi_{\mathcal{H}}, \delta_{\mathcal{H}}}(\sigma)$ is the preimage (with

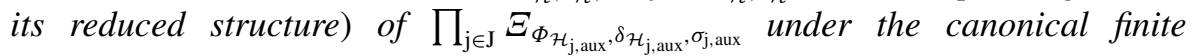
morphism (8.18) of schemes, and the induced canonical morphism

$$
\overrightarrow{\mathfrak{X}}_{\Phi_{\mathcal{H}}, \delta_{\mathcal{H}}, \sigma} \rightarrow \mathfrak{X}_{\left(\Phi_{\mathcal{H}_{j}, \text { aux }}, \delta_{\mathcal{H}_{\mathrm{j}, \text { aux }},}, \sigma_{\mathrm{j}, \text { aux }}\right)_{j \in \mathrm{J}}}
$$

of formal schemes is finite (we cannot expect $\vec{\Xi}_{\Phi_{\mathcal{H}}, \delta_{\mathcal{H}}, \sigma}$ to be the schematic

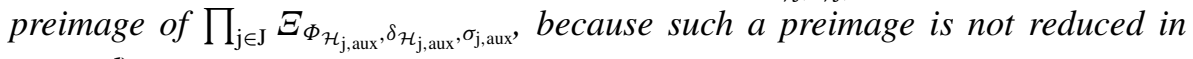
general).

Proof. It suffices to show that $\vec{\Xi}_{\Phi_{\mathcal{H}}, \delta_{\mathcal{H}}, \sigma}$ is the preimage (with its reduced structure) of $\prod_{\mathrm{j} \in \mathrm{J}} \Xi_{\Phi_{\mathcal{H}_{\mathrm{j}, \text { aux }}}, \delta_{\mathcal{H}_{\mathrm{j}, \text { aux }}, \sigma_{\mathrm{j}, \text { aux }}}}$ under (8.18).

By definition, the closed subscheme $\vec{\Xi}_{\Phi_{\mathcal{H}}, \delta_{\mathcal{H}}, \sigma}$ of $\vec{\Xi}_{\Phi_{\mathcal{H}}, \delta_{\mathcal{H}}}(\sigma)$ is defined by the $\mathscr{O}_{\vec{\Xi}_{\Phi_{\mathcal{H}}, \delta \mathcal{H}}(\sigma)}$-ideal corresponding to the $\mathscr{O}_{\vec{C}_{\Phi_{\mathcal{H}}, \delta_{\mathcal{H}}}}$-submodule $\bigoplus_{\ell \in \sigma_{0}^{\vee}} \vec{\Psi}_{\Phi_{\mathcal{H}}, \delta_{\mathcal{H}}}(\ell)$ of the $\mathscr{O}_{\vec{C}_{\Phi_{\mathcal{H}}, \delta_{\mathcal{H}}}}$-algebra $\bigoplus_{\ell \in \sigma^{\vee}} \vec{\Psi}_{\Phi_{\mathcal{H}}, \delta_{\mathcal{H}}}(\ell)$. Similarly, for 
each $\mathrm{j} \in \mathrm{J}$, the closed subscheme $\boldsymbol{\Xi}_{\Phi_{\mathcal{H}_{\mathrm{j}, \text { aux }}}, \delta \mathcal{H}_{\mathrm{j}, \text { aux }}, \sigma_{\mathrm{j} \text {,aux }}}$ of $\boldsymbol{\Xi}_{\Phi_{\mathcal{H}_{\mathrm{j}, \text { aux }}}, \delta_{\mathcal{H}_{\mathrm{j}, \text { aux }}}}\left(\sigma_{\mathrm{j} \text {, aux }}\right)$ is

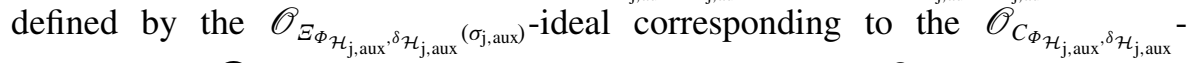
submodule $\bigoplus_{\ell_{\mathrm{j}, \text { aux }} \in\left(\sigma_{\mathrm{j}, \text { aux }}\right)_{0}^{\vee}} \Psi_{\Phi_{\mathcal{H}_{\mathrm{j}, \text { aux }}}, \delta \mathcal{H}_{\mathrm{j}, \text { aux }}}\left(\ell_{\mathrm{j}, \text { aux }}\right) \quad$ of the $\mathscr{O}_{C_{\Phi_{\mathcal{H}_{\mathrm{j}, \text { aux }}}, \delta \mathcal{H}_{\mathrm{j}, \text { aux }}} \text {-algebra }}$ $\bigoplus_{\ell_{\mathrm{j}, \text { aux }} \in \sigma_{\mathrm{j}, \text { aux }}^{\vee}} \Psi_{\Phi_{\mathcal{H}_{\mathrm{j}, \text { aux }}}, \delta_{\mathcal{H}_{\mathrm{j}, \text { aux }}}}\left(\ell_{\mathrm{j} \text {, aux }}\right)$. Since

$$
\left(\prod_{\mathrm{j} \in \mathrm{J}} \sigma_{\mathrm{j}, \text { aux }}\right)^{\vee}=\prod_{\mathrm{j} \in \mathrm{J}} \sigma_{\mathrm{j}, \text { aux }}^{\vee}
$$

and

$$
\left(\prod_{\mathrm{j} \in \mathrm{J}} \sigma_{\mathrm{j}, \text { aux }}\right)_{0}^{\vee}=\left(\prod_{\mathrm{j} \in \mathrm{J}} \sigma_{\mathrm{j}, \text { aux }}^{\vee}\right)-\left(\prod_{\mathrm{j} \in \mathrm{J}} \sigma_{\mathrm{j}, \text { aux }}^{\perp}\right)
$$

(where '-' means set subtraction), the closed subscheme $\prod_{\mathrm{j} \in \mathrm{J}} \Xi_{\Phi_{\mathcal{H}_{\mathrm{j}, \text { aux }}, \delta_{\mathcal{H}} \text {, aux }}, \sigma_{\mathrm{j} \text {, aux }}}$

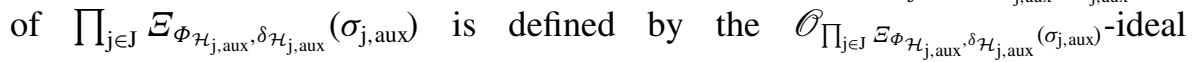

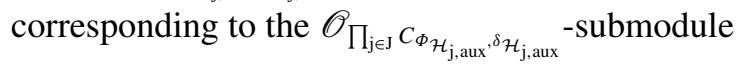

$$
\bigoplus_{\left(\left(\ell_{\mathrm{j}, \text { aux }}\right)_{\mathrm{j} \in \mathrm{J}}\right) \in\left(\prod_{\mathrm{j} \in \mathrm{J}} \sigma_{\mathrm{j}, \text { aux }}\right)_{0}^{\vee}}\left(\bigotimes_{\mathrm{j} \in \mathrm{J}} \Psi_{\left.\Phi_{\mathcal{H}_{\mathrm{j}, \text { aux }}, \delta \mathcal{H}_{\mathrm{j}, \text { aux }}}\left(\ell_{\mathrm{j}, \text { aux }}\right)\right)}\right.
$$

of the $\mathscr{O}_{\prod_{\mathrm{j} \in \mathrm{J}} C_{\Phi_{\mathcal{H}}, \text { aux }},{ }^{,} \mathcal{H}_{\mathrm{j}, \text { aux }}}$-algebra

$$
\bigoplus_{\left(\left(\ell_{\mathrm{j}, \text { aux }}\right)_{\mathrm{j} \in \mathrm{J}}\right) \in\left(\prod_{\mathrm{j} \in \mathrm{J}} \sigma_{\mathrm{j}, \text { aux }}\right)^{\vee}}\left(\bigotimes_{\mathrm{j} \in \mathrm{J}} \Psi_{\Phi_{\mathcal{H}_{\mathrm{j}, \text { aux }}}, \delta_{\mathcal{H}_{\mathrm{j}, \text { aux }}}}\left(\ell_{\mathrm{j}, \text { aux }}\right)\right) .
$$

Since the $\mathbb{Q}_{>0}$-span of $\sigma_{0}^{\vee}$ (respectively $\sigma^{\perp}$ ) is the image of the $\mathbb{Q}_{>0}$-span of $\left(\prod_{\mathrm{j} \in \mathrm{J}} \sigma_{\mathrm{j} \text {,aux }}\right)_{0}^{\vee}$ (respectively $\left(\prod_{\mathrm{j} \in \mathrm{J}} \sigma_{\mathrm{j} \text {,aux }}\right)^{\perp}$ ) under the map (5.21) (cf. the proof of Proposition 8.14), the desired assertion follows from the last paragraph of Proposition 8.7.

As explained in [30, Section 6.2.5], using the language of relative schemes

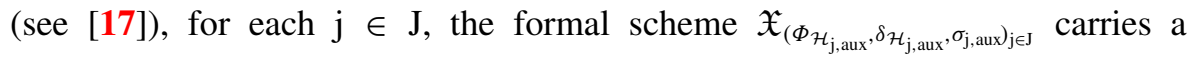
tautological tuple

$$
\begin{aligned}
& \left(\mathrm{Z}_{\mathcal{H}_{\mathrm{j}, \text { aux }}},\left(X_{\mathrm{j}, \text { aux }}, Y_{\mathrm{j} \text {,aux }}, \phi_{\mathrm{j}, \text { aux }}, \varphi_{-2, \mathcal{H}_{\mathrm{j}, \text { aux }}}^{\sim}, \varphi_{0, \mathcal{H}_{\mathrm{j}, \text { aux }}}^{\sim}\right),\right. \\
& \left.\left(B_{\mathrm{j}, \text { aux }}, \lambda_{B_{\mathrm{j}, \text { aux }}}, i_{B_{\mathrm{j}, \text { aux }}}, \varphi_{-1, \mathcal{H}_{\mathrm{j}, \text { aux }}}\right), \delta_{\mathcal{H}_{\mathrm{j}, \text { aux }}},\left(c_{\mathcal{H}_{\mathrm{j}, \text { aux }}}, c_{\mathcal{H}_{\mathrm{j}, \text { aux }}}^{\vee}, \tau_{\mathcal{H}_{\mathrm{j}, \text { aux }}}\right)\right),
\end{aligned}
$$

where $\left(\varphi_{-2, \mathcal{H}_{\mathrm{j}, \text { aux }}}^{\sim}, \tilde{\varphi_{0, \mathcal{H}_{\mathrm{j}, \text { aux }}}^{\sim}}\right)$ induces the $\left(\varphi_{-2, \mathcal{H}_{\mathrm{j}, \text { aux }}}, \varphi_{0, \mathcal{H}_{\mathrm{j}, \text { aux }}}\right)$ in $\Phi_{\mathcal{H}_{\mathrm{j}, \text { aux }}}$. Let us denote the pullback of (8.22) to $\overrightarrow{\mathfrak{X}}_{\Phi_{\mathcal{H}}, \delta_{\mathcal{H}}, \sigma}$ by

$$
\begin{aligned}
& \left(\mathrm{Z}_{\mathcal{H}_{\mathrm{j}, \text { aux }}},\left(X_{\mathrm{j}, \text { aux }}, Y_{\mathrm{j}, \text { aux }}, \phi_{\mathrm{j}, \text { aux }}, \vec{\varphi}_{-2, \mathcal{H}_{\mathrm{j}, \text { aux }}}^{\sim}, \vec{\varphi}_{0, \mathcal{H}_{\mathrm{j}, \text { aux }}}^{\sim}\right),\right. \\
& \left.\left(\vec{B}_{\mathrm{j}, \text { aux }}, \lambda_{\vec{B}_{\mathrm{j}, \text { aux }}}, i_{\vec{B}_{\mathrm{j}, \text { aux }}}, \vec{\varphi}_{-1, \mathcal{H}_{\mathrm{j}, \text { aux }}}\right), \delta_{\mathcal{H}_{\mathrm{j}, \text { aux }}},\left(\vec{c}_{\mathcal{H}_{\mathrm{j}, \text { aux }}}, \vec{c}_{\mathcal{H}_{\mathrm{j}, \text { aux }}}^{\vee}, \vec{\tau}_{\mathcal{H}_{\mathrm{j}, \text { aux }}}\right)\right) .
\end{aligned}
$$


Similarly, for each $\mathrm{j} \in \mathrm{J}$, the formal scheme $\mathfrak{X}_{\Phi_{\mathcal{H}}, \delta \mathcal{H}, \sigma} \cong \mathfrak{X}_{\Phi_{\mathcal{H}_{\mathrm{j}}}, \delta_{\mathcal{H}_{\mathrm{j}}}, \sigma_{\mathrm{j}}}($ see (3.20)) carries a tautological tuple

$$
\begin{aligned}
& \left(\mathrm{Z}_{\mathcal{H}_{\mathrm{j}}},\left(X_{\mathrm{j}}, Y_{\mathrm{j}}, \phi_{\mathrm{j}}, \varphi_{-2, \mathcal{H}_{\mathrm{j}}}^{\sim}, \varphi_{0, \mathcal{H}_{\mathrm{j}}}^{\sim}\right),\right. \\
& \left.\left(B_{\mathrm{j}}, \lambda_{B_{\mathrm{j}}}, i_{B_{\mathrm{j}}}, \varphi_{-1, \mathcal{H}_{\mathrm{j}}}\right), \delta_{\mathcal{H}_{\mathrm{j}}},\left(c_{\mathcal{H}_{\mathrm{j}}}, c_{\mathcal{H}_{\mathrm{j}}}^{\vee}, \tau_{\mathcal{H}_{\mathrm{j}}}\right)\right),
\end{aligned}
$$

where $\left(\varphi_{-2, \mathcal{H}_{\mathrm{j}}}^{\sim}, \varphi_{0, \mathcal{H}_{\mathrm{j}}}^{\sim}\right)$ induces the $\left(\varphi_{-2, \mathcal{H}_{\mathrm{j}}}, \varphi_{0, \mathcal{H}_{\mathrm{j}}}\right)$ in $\Phi_{\mathcal{H}_{\mathrm{j}}}$, which extends to a tautological tuple

$$
\begin{aligned}
& \left(Z_{\mathcal{H}_{\mathrm{j}}},\left(X_{\mathrm{j}}, Y_{\mathrm{j}}, \phi_{\mathrm{j}}, \vec{\varphi}_{-2, \mathcal{H}_{\mathrm{j}}}^{\sim}, \vec{\varphi}_{0, \mathcal{H}_{\mathrm{j}}}^{\sim}\right),\right. \\
& \left.\left(\vec{B}_{\mathrm{j}}, \lambda_{\vec{B}_{\mathrm{j}}}, i_{\vec{B}_{\mathrm{j}}}, \vec{\varphi}_{-1, \mathcal{H}_{\mathrm{j}}}\right), \delta_{\mathcal{H}_{\mathrm{j}}},\left(\vec{c}_{\mathcal{H}_{\mathrm{j}}}, \vec{c}_{\mathcal{H}_{\mathrm{j}}}^{\vee}, \vec{\tau}_{\mathcal{H}_{\mathrm{j}}}\right)\right)
\end{aligned}
$$

over the formal scheme $\overrightarrow{\mathfrak{X}}_{\Phi_{\mathcal{H}}, \delta_{\mathcal{H}}, \sigma}$, where $\left(\vec{\varphi}_{-2, \mathcal{H}_{\mathrm{j}}}^{\sim}, \vec{\varphi}_{0, \mathcal{H}_{\mathrm{j}}}^{\sim}\right)$ and $\vec{\varphi}_{-1, \mathcal{H}_{\mathrm{j}}}$ are defined only over $\mathfrak{X}_{\Phi_{\mathcal{H}}, \delta \mathcal{H}, \sigma} \cong \mathfrak{X}_{\Phi_{\mathcal{H}_{\mathrm{j}}}, \delta \mathcal{H}_{\mathrm{j}}, \sigma_{\mathrm{j}}}$, and where $\left(\vec{c}_{\mathcal{H}_{\mathrm{j}}}, \vec{c}_{\mathcal{H}_{\mathrm{j}}}^{\vee}, \vec{\tau}_{\mathcal{H}_{\mathrm{j}}}\right)$ is defined only over $\mathfrak{X}_{\Phi_{\mathcal{H}}, \delta_{\mathcal{H}}, \sigma} \cong \mathfrak{X}_{\Phi_{\mathcal{H}_{\mathrm{j}}, \delta \mathcal{H}_{\mathrm{j}}, \sigma_{\mathrm{j}}}}$ but nevertheless induces a tuple $\left(\vec{c}_{\mathrm{j}}, \vec{c}_{\mathrm{j}}^{\vee}, \vec{\tau}_{\mathrm{j}}\right)$ defined over all of $\overrightarrow{\mathfrak{X}}_{\Phi_{\mathcal{H}}, \delta_{\mathcal{H}}, \sigma}$, which in turn induces the tuple $\left(\vec{c}_{\mathrm{j}, \text { aux }}, \vec{c}_{\mathrm{j} \text {,aux }}^{\vee}, \vec{\tau}_{\mathrm{j} \text {,aux }}\right)$ induced by $\left(\vec{c}_{\mathcal{H}_{\mathrm{j}, \text { aux }}}\right.$, $\left.\vec{c}_{\mathcal{H}_{\mathrm{j}, \text { aux }}}^{\vee}, \vec{\tau}_{\mathcal{H}_{\mathrm{j}, \text { aux }}}\right)$.

By construction, (8.23) is induced by (8.25) in the following sense.

(1) $\left(\mathrm{Z}_{\mathcal{H}_{\mathrm{j}, \text { aux }}}, \Phi_{\mathcal{H}_{\mathrm{j}, \text { aux }}}=\left(X_{\mathrm{j} \text {,aux }}, Y_{\mathrm{j} \text {,aux }}, \phi_{\mathrm{j}, \text { aux }}, \varphi_{-2, \mathcal{H}_{\mathrm{j}, \text { aux }}}, \varphi_{0, \mathcal{H}_{\mathrm{j}, \text { aux }}}\right), \delta_{\mathcal{H}_{\mathrm{j} \text {,aux }}}\right)$ is induced by $\left(\mathrm{Z}_{\mathcal{H}_{\mathrm{j}}}, \Phi_{\mathcal{H}_{\mathrm{j}}}=\left(X_{\mathrm{j}}, Y_{\mathrm{j}}, \phi_{\mathrm{j}}, \varphi_{-2, \mathcal{H}_{\mathrm{j}}}, \varphi_{0, \mathcal{H}_{\mathrm{j}}}\right), \delta_{\mathcal{H}_{\mathrm{j}}}\right)$ via $(5.8)$.

(2) $\left(\vec{B}_{\mathrm{j}, \text { aux }}, \lambda_{\vec{B}_{\mathrm{j}, \text { aux }}}, i_{\overrightarrow{\mathrm{b}}_{\mathrm{j}, \text { aux }}}, \vec{\varphi}_{-1, \mathcal{H}_{\mathrm{j}, \text { aux }}}\right)$ is induced by $\left(\vec{B}_{\mathrm{j}}, \lambda_{\vec{B}_{\mathrm{j}}}, i_{\vec{B}_{\mathrm{j}}}, \varphi_{-1, \mathcal{H}_{\mathrm{j}}}\right)$.

(3) $\left(\vec{\varphi}_{-2, \mathcal{H}_{\mathrm{j}, \text { aux }}}^{\sim}, \vec{\varphi}_{0, \mathcal{H}_{\mathrm{j}, \text { aux }}}^{\sim}\right)$ is induced by $\left(\vec{\varphi}_{-2, \mathcal{H}_{\mathrm{j}}}^{\sim}, \vec{\varphi}_{0, \mathcal{H}_{\mathrm{j}}}^{\sim}\right)$ by forgetting the factors at $p$ and by forming the $\mathcal{H}_{\mathrm{j} \text {,aux }}$-orbits.

(4) $\left(\vec{c}_{\mathcal{H}_{\mathrm{j}, \text { aux }}}, \vec{c}_{\mathcal{H}_{\mathrm{j}, \text { aux }}}^{\vee}, \vec{\tau}_{\mathcal{H}_{\mathrm{j} \text {,aux }}}\right)$ is also induced by $\left(\vec{c}_{\mathcal{H}_{\mathrm{j}}}, \vec{c}_{\mathcal{H}_{\mathrm{j}}}^{\vee}, \vec{\tau}_{\mathcal{H}_{\mathrm{j}}}\right)$ by forgetting the

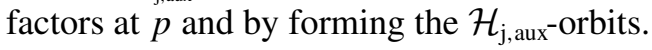

As explained in [30, Section 6.2.5], the tautological tuples (8.22), (8.23), (8.24), and (8.25) define the respective Mumford families

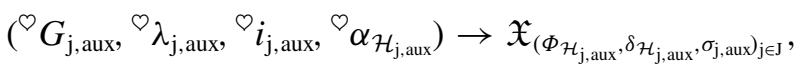

$$
\begin{aligned}
& \left({ }^{\odot} \vec{G}_{\mathrm{j}, \text { aux }},{ }^{\odot} \vec{\lambda}_{\mathrm{j}, \text { aux }},{ }^{\circ}{\overrightarrow{i_{\mathrm{j}} \text { aux }}},{ }^{\odot} \vec{\alpha}_{\mathcal{H}_{\mathrm{j}, \text { aux }}}\right) \rightarrow \overrightarrow{\mathfrak{X}}_{\Phi_{\mathcal{H}}, \delta_{\mathcal{H}}, \sigma}, \\
& \left({ }^{\odot} G_{\mathrm{j}},{ }^{\odot} \lambda_{\mathrm{j}},{ }^{\odot} i_{\mathrm{j}},{ }^{\odot} \alpha_{\mathcal{H}_{\mathrm{j}}}\right) \rightarrow \mathfrak{X}_{\Phi_{\mathcal{H}}, \delta_{\mathcal{H}}, \sigma} \cong \mathfrak{X}_{\Phi_{\mathcal{H}_{\mathrm{j}}}, \delta_{\mathcal{H}_{\mathrm{j}}}, \sigma_{\mathrm{j}}},
\end{aligned}
$$

and

$$
\left({ }^{\odot} \vec{G}_{\mathrm{j}}, \stackrel{\odot}{{ }^{\prime}} \vec{\lambda}_{\mathrm{j}}, \stackrel{\odot}{\vec{i}_{\mathrm{j}}},{ }^{\odot} \vec{\alpha}_{\mathcal{H}_{\mathrm{j}}}\right) \rightarrow \overrightarrow{\mathfrak{X}}_{\Phi_{\mathcal{H}}, \delta_{\mathcal{H}}, \sigma},
$$

where ${ }^{\ominus} \vec{\alpha}_{\mathcal{H}_{\mathrm{j}}}$ is defined only over $\overrightarrow{\mathfrak{X}}_{\Phi_{\mathcal{H}}, \delta_{\mathcal{H}}, \sigma} \otimes_{\mathbb{Z}} \mathbb{Q} \cong \mathfrak{X}_{\Phi_{\mathcal{H}}, \delta_{\mathcal{H}}, \sigma} \cong \mathfrak{X}_{\Phi_{\mathcal{H}_{\mathrm{j}}}, \delta_{\mathcal{H}_{\mathrm{j}}}, \sigma_{\mathrm{j}}}$. 
REMARK 8.30. Although $\sigma$ was assumed to be smooth in [30, Section 6.2.5], the argument there only requires the excellent normality of $\Xi_{\Phi_{\mathcal{H}}, \delta \mathcal{H}}(\sigma)$ and $\vec{\Xi}_{\Phi_{\mathcal{H}}, \delta_{\mathcal{H}}}(\sigma)$, which we have shown in Proposition 8.14.

For all $\mathrm{j} \in \mathrm{J}$, by the functoriality of Mumford's construction, we know that (8.27) is canonically isomorphic to the pullback of (8.26) under (8.21); that (8.28) is canonically isomorphic to the pullback of (8.29); and that (8.27) is induced by (8.29). By the universal property of $\mathrm{M}_{\mathcal{H}_{\mathrm{j}, \text { aux }}, \Sigma_{\mathrm{j}, \text { aux }}}^{\text {tor }}$ as in $[\mathbf{3 0}$, Theorem 6.4.1.1(6)], the Mumford families (8.26), for all $\mathrm{j} \in \mathrm{J}$, induce a canonical morphism

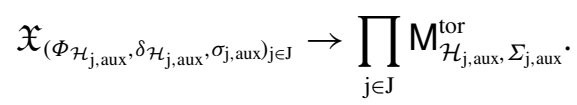

Similarly, since (8.27) is canonically isomorphic to the pullback of (8.26) for each $\mathrm{j} \in \mathrm{J}$, the Mumford families (8.27), for all $\mathrm{j} \in \mathrm{J}$, induce a morphism

$$
\overrightarrow{\mathfrak{X}}_{\Phi_{\mathcal{H}}, \delta_{\mathcal{H}}, \sigma} \rightarrow \prod_{\mathrm{j} \in \mathrm{J}} \mathrm{M}_{\mathcal{H}_{\mathrm{j}, \text { aux }}, \Sigma_{\mathrm{j}, \text { aux }}}^{\text {tor }}
$$

which coincides with the composition of (8.21) with (8.31). Since (8.27) is induced by (8.29) for each $\mathrm{j} \in \mathrm{J}$, by the universal property of $\overrightarrow{\mathrm{M}}_{\mathcal{H}, \Sigma}^{\text {tor }}$ as in Proposition 7.14, the morphism (8.32) lifts to a morphism

$$
\overrightarrow{\mathfrak{X}}_{\Phi_{\mathcal{H}}, \delta_{\mathcal{H}}, \sigma} \rightarrow \overrightarrow{\mathrm{M}}_{\mathcal{H}, \Sigma}^{\text {tor }},
$$

whose composition with (7.8) is (8.32).

\section{Stratifications of toroidal compactifications}

The main goal of this section is to show that an analog of [30, Theorem 6.4.1.1(2)] is true for $\overrightarrow{\mathrm{M}}_{\mathcal{H}, \Sigma}^{\text {tor }}$ (see Theorem 9.13 below).

DeFinition 9.1. For each $\left(\Phi_{\mathcal{H}}, \delta_{\mathcal{H}}, \sigma\right)$ as in [30, Definition 6.2.6.1] such that $\sigma \subset \mathbf{P}_{\Phi_{\mathcal{H}}}^{+}$and $\sigma \in \Sigma_{\Phi_{\mathcal{H}}}$, let $\vec{Z}_{\left[\left(\Phi_{\mathcal{H}}, \delta_{\mathcal{H}}, \sigma\right)\right]}$ denote the subset of $\overrightarrow{\mathrm{M}}_{\mathcal{H}, \Sigma}^{\text {tor }}$ consisting of all points $t$ satisfying the follow property: for any morphism $\operatorname{Spec}(V) \rightarrow \overrightarrow{\mathrm{M}}_{\mathcal{H}, \Sigma}^{\text {tor }}$, where $V$ is a complete discrete valuation ring with fraction field $K$, algebraically closed residue field $k$, and discrete valuation $v$, which maps the generic point $\operatorname{Spec}(K)$ to some maximal point (see $[15,0,2.1 .2]$ ) of $\mathrm{M}_{\mathcal{H}}$ and maps the special point $\operatorname{Spec}(k)$ to $t$, there exist some $\left(\mathrm{Z}_{\mathcal{H}}^{\ddagger}, \Phi_{\mathcal{H}}^{\ddagger}, \delta_{\mathcal{H}}^{\ddagger}\right)$ and some $\sigma^{\ddagger} \in \Sigma_{\Phi_{\mathcal{H}}^{\ddagger}}$ as in Theorem 7.14, where $\sigma^{\ddagger}$ is minimal among all choices, such that $\left[\left(\Phi_{\mathcal{H}}^{\ddagger}, \delta_{\mathcal{H}}^{\ddagger}\right.\right.$, $\left.\left.\sigma^{\ddagger}\right)\right]=\left[\left(\Phi_{\mathcal{H}}, \delta_{\mathcal{H}}, \sigma\right)\right]$ (that is, $\left(\Phi_{\mathcal{H}}^{\ddagger}, \delta_{\mathcal{H}}^{\ddagger}, \sigma^{\ddagger}\right)$ and $\left(\Phi_{\mathcal{H}}, \delta_{\mathcal{H}}, \sigma\right)$ are equivalent as in [30, Definition 6.2.6.1]). 
REMARK 9.2. As explained in the proof of [30, Proposition 6.3.3.11], the requirement that $\sigma^{\ddagger}$ is minimal among all choices implies that $v \circ B^{\ddagger} \in \sigma^{\ddagger}$, not just in the closure $\bar{\sigma}^{\ddagger}$.

LEMMA 9.3. The underlying set of $\overrightarrow{\mathrm{M}}_{\mathcal{H}, \Sigma}^{\text {tor }}$ is the disjoint union of $\vec{Z}_{\left[\left(\Phi_{\mathcal{H}}, \delta_{\mathcal{H}}, \sigma\right)\right]}$, with $\left[\left(\Phi_{\mathcal{H}}, \delta_{\mathcal{H}}, \sigma\right)\right]$ running through a complete set of equivalence classes of $\left(\Phi_{\mathcal{H}}, \delta_{\mathcal{H}}\right.$, $\sigma)$ (as in [30, Definition 6.2.6.1]) with $\sigma \subset \mathbf{P}_{\Phi_{\mathcal{H}}}^{+}$and $\sigma \in \Sigma_{\Phi_{\mathcal{H}}} \in \Sigma$.

Proof. The fact that $\overrightarrow{\mathrm{M}}_{\mathcal{H}, \Sigma}^{\text {tor }}$ is the union as in the statement of the lemma follows from Theorem 7.14. Since $\overrightarrow{\mathrm{M}}_{\mathcal{H}, \Sigma}^{\text {tor }}$ is noetherian and normal, given any point $t$ of $\overrightarrow{\mathrm{M}}_{\mathcal{H}, \Sigma}^{\text {tor }}$, all morphisms $\operatorname{Spec}(V) \rightarrow \overrightarrow{\mathrm{M}}_{\mathcal{H}, \Sigma}^{\text {tor }}$ as in Definition 9.1 (for some $V$ ) that map the special point to $t$ must map the generic point to the same maximal point $\eta$ of $\mathrm{M}_{\mathcal{H}}$. Let us denote by $\left(A_{\eta}, \lambda_{\eta}, i_{\eta}, \alpha_{\mathcal{H}, \eta}\right)$ the object parameterized by the canonical morphism $\eta \rightarrow \mathrm{M}_{\mathcal{H}}$. Since the assignment of $\left[\left(\Phi_{\mathcal{H}}^{\ddagger}, \delta_{\mathcal{H}}^{\ddagger}, \sigma^{\ddagger}\right)\right]$ to a morphism $\operatorname{Spec}(V) \rightarrow \overrightarrow{\mathrm{M}}_{\mathcal{H}, \Sigma}^{\text {tor }}$ as in Theorem 7.14 is determined by the degeneration of the pullback of $\left(A_{\eta}, \lambda_{\eta}, i_{\eta}, \alpha_{\mathcal{H}, \eta}\right)$ to $\operatorname{Spec}(K)$, which is unchanged under faithfully flat extensions of discrete valuation rings $V$, it follows that $\left[\left(\Phi_{\mathcal{H}}^{\ddagger}\right.\right.$, $\left.\left.\delta_{\mathcal{H}}^{\ddagger}, \sigma^{\ddagger}\right)\right]$ depends only on $t$. Hence the union in the statement of the lemma is disjoint, as desired.

LEMMA 9.4. For each $\left(\Phi_{\mathcal{H}}, \delta_{\mathcal{H}}, \sigma\right)$ (as in [30, Definition 6.2.6.1]) such that $\sigma \subset$ $\mathbf{P}_{\Phi_{\mathcal{H}}}^{+}$and $\sigma \in \Sigma_{\Phi_{\mathcal{H}}} \in \Sigma$, the subset $\overrightarrow{\mathrm{Z}}_{\left[\left(\Phi_{\mathcal{H}}, \delta_{\mathcal{H}}, \sigma\right)\right]}$ of $\overrightarrow{\mathrm{M}}_{\mathcal{H}, \Sigma}^{\text {tor }}$ is the (set-theoretic) image of the morphism

$$
\vec{\Xi}_{\Phi_{\mathcal{H}}, \delta_{\mathcal{H}}, \sigma} \rightarrow \overrightarrow{\mathrm{M}}_{\mathcal{H}, \Sigma}^{\text {tor }}
$$

induced by (8.33).

Proof. Since (8.33) is induced by the universal property of $\overrightarrow{\mathrm{M}}_{\mathcal{H}, \Sigma}^{\text {tor }}$, the image of (9.5) lies in $\vec{Z}_{\left[\left(\Phi_{\mathcal{H}}, \delta_{\mathcal{H}}, \sigma\right)\right]}$ by definition. Conversely, let $t$ be any point of $\overrightarrow{\mathrm{Z}}_{\left[\left(\Phi_{\mathcal{H}}, \delta_{\mathcal{H}}, \sigma\right)\right]}$, also viewed as a point of $\mathrm{M}_{\mathcal{H}, \Sigma}^{\text {tor }}$. Let

$$
\operatorname{Spec}(V) \rightarrow \mathrm{M}_{\mathcal{H}, \Sigma}^{\text {tor }}
$$

be as in Definition 9.1, which maps the special point to $t$, such that the pullback of the tautological object over $\mathrm{M}_{\mathcal{H}}$ to the generic point of $\operatorname{Spec}(V)$ extends to a degenerating family $\left(G^{\dagger}, \lambda^{\dagger}, i^{\dagger}, \alpha_{\mathcal{H}}^{\dagger}\right)$ of type $\mathrm{M}_{\mathcal{H}}$ over $\operatorname{Spec}(V)$, which defines an object of $\mathrm{DEG}_{\mathrm{PEL}, \mathrm{M}_{\mathcal{H}}}(V)$ corresponding to an object in $\operatorname{DD}_{\mathrm{PEL}, \mathrm{M}_{\mathcal{H}}}(V)$ under [30, Theorem 5.3.1.19]. For each $\mathrm{j} \in \mathrm{J}$, as explained in the proof of Theorem 7.14, the pullback of the tautological object over $M_{\mathcal{H}_{j}}$ to the generic 
point of $\operatorname{Spec}(V)$ also extends to a degenerating family $\left(G_{\mathrm{j}}^{\dagger}, \lambda_{\mathrm{j}}^{\dagger}, i_{\mathrm{j}}^{\dagger}, \alpha_{\mathcal{H}_{\mathrm{j}}}^{\dagger}\right)$ of type $\mathrm{M}_{\mathcal{H}_{\mathrm{j}}}$ over $\operatorname{Spec}(V)$, which defines an object of $\mathrm{DEG}_{\mathrm{PEL}, \mathrm{M}_{\mathcal{H}_{\mathrm{j}}}}(V)$ corresponding to an object in $\operatorname{DD}_{\mathrm{PEL}, \mathrm{M}_{\mathcal{H}_{\mathrm{j}}}}(V)$ under [30, Theorem 5.3.1.19]. Since $t$ is a point of $\vec{Z}_{\left[\left(\Phi_{\mathcal{H}}, \delta_{\mathcal{H}}, \sigma\right)\right]}$, these objects in $\operatorname{DD}_{\mathrm{PEL}, \mathrm{M}_{\mathcal{H}}}(V)$, for all $\mathrm{j} \in \mathrm{J}$, induce objects parameterized by $\overrightarrow{\mathfrak{X}}_{\Phi_{\mathcal{H}}, \delta_{\mathcal{H}}, \sigma}$ as in (8.25), so that the degenerating families $\left(G_{\mathrm{j}}^{\dagger}, \lambda_{\mathrm{j}}^{\dagger}, i_{\mathrm{j}}^{\dagger}, \alpha_{\mathcal{H}_{\mathrm{j}}}^{\dagger}\right)$ are isomorphic to the respective pullbacks of the Mumford families (8.29), for all $\mathrm{j} \in \mathrm{J}$, under a uniquely determined morphism

$$
\operatorname{Spf}(V) \rightarrow \overrightarrow{\mathfrak{X}}_{\Phi_{\mathcal{H}}, \delta_{\mathcal{H}}, \sigma}
$$

Since (8.33) is induced by the universal property of $\overrightarrow{\mathrm{M}}_{\mathcal{H}, \Sigma}^{\text {tor }}$, its precomposition with (9.7) is induced by (9.6). Therefore $t$ lies in the image of (9.5), as desired.

LEMMA 9.8. With the setting as above, suppose that $\Sigma^{\prime}$ is any compatible choice of admissible smooth rational polyhedral cone decomposition data as in [30, Definition 6.3.3.4], which defines a smooth toroidal compactification $\mathrm{M}_{\mathcal{H}, \Sigma^{\prime}}^{\text {tor }}$ as in [30, Theorem 6.4.1.1], and suppose that $\Sigma^{\prime}$ is a refinement of $\Sigma$ as in [30, Definition 6.4.2.2] (with the roles of $\Sigma$ and $\Sigma^{\prime}$ there interchanged; such $\Sigma^{\prime}$ always exists by compatibly refining the cone decompositions $\Sigma_{\Phi_{\mathcal{H}}}$ as in the proof of [30, Proposition 6.3.3.5]). Let $\mathrm{M}_{\mathcal{H}, \Sigma}^{\text {tor }}:=\overrightarrow{\mathrm{M}}_{\mathcal{H}, \Sigma}^{\text {tor }} \otimes_{\mathbb{Z}} \mathbb{Q}$, which we view as an open subscheme of $\overrightarrow{\mathrm{M}}_{\mathcal{H}, \Sigma}^{\text {tor }}$. Then there exists a canonical proper surjective morphism

$$
\mathrm{M}_{\mathcal{H}, \Sigma^{\prime}}^{\text {tor }} \rightarrow \mathrm{M}_{\mathcal{H}, \Sigma}^{\mathrm{tor}}
$$

mapping $\mathrm{Z}_{\left[\left(\Phi_{\mathcal{H}}, \delta_{\mathcal{H}}, \tau\right)\right]}$ to

$$
\mathrm{Z}_{\left[\left(\Phi_{\mathcal{H}}, \delta_{\mathcal{H}}, \sigma\right)\right]}:=\overrightarrow{\mathrm{Z}}_{\left[\left(\Phi_{\mathcal{H}}, \delta_{\mathcal{H}}, \sigma\right)\right]} \cap \mathrm{M}_{\mathcal{H}, \Sigma}^{\mathrm{tor}}
$$

whenever $\tau \in \Sigma_{\Phi_{\mathcal{H}}}^{\prime} \in \Sigma^{\prime}$ is contained in $\sigma \in \Sigma_{\Phi_{\mathcal{H}}} \in \Sigma$ in $\mathbf{P}_{\Phi_{\mathcal{H}}}^{+}$. Moreover, each $\mathrm{Z}_{\left[\left(\Phi_{\mathcal{H}}, \delta_{\mathcal{H}}, \sigma\right)\right]}$ is the union of the images of all such $\mathrm{Z}_{\left[\left(\Phi_{\mathcal{H}}, \delta_{\mathcal{H}}, \tau\right)\right]}$. In particular, the open dense stratum $\mathrm{Z}_{[(0,0,\{0\})]}$ of $\mathrm{M}_{\mathcal{H}, \Sigma}^{\text {tor }}$ is the isomorphic image of the open dense stratum $\mathrm{Z}_{[(0,0,\{0\})]}=\mathrm{M}_{\mathcal{H}}$ of $\mathrm{M}_{\mathcal{H}, \Sigma^{\prime}}^{\text {tor }}$, which must coincide with the open dense subscheme $\mathrm{M}_{\mathcal{H}} \cong \overrightarrow{\mathrm{M}}_{\mathcal{H}} \otimes_{\mathbb{Z}} \mathbb{Q}$ of $\mathrm{M}_{\mathcal{H}, \Sigma}^{\text {tor }}=\overrightarrow{\mathrm{M}}_{\mathcal{H}, \Sigma}^{\text {tor }} \otimes_{\mathbb{Z}} \mathbb{Q}($ see Lemma 7.9).

Consequently, the subsets $\mathrm{Z}_{\left[\left(\Phi_{\mathcal{H}}, \delta_{\mathcal{H}}, \sigma\right)\right]}$ of $\mathrm{M}_{\mathcal{H}, \Sigma}^{\text {tor }}$, with $\left[\left(\Phi_{\mathcal{H}}, \delta_{\mathcal{H}}, \sigma\right)\right]$ running through all equivalence classes as in [30, Definition 6.2.6.1], are locally closed and define a stratification of $\mathrm{M}_{\mathcal{H}, \Sigma}^{\text {tor }}$ as in [30, Theorem 6.4.1.1(2)] (with incidence relations described as in the second paragraph there, and with $\mathrm{M}_{\mathcal{H}} \cong \mathrm{Z}_{[(0,0,\{0\})]}$ being an open dense stratum). Then each $\mathrm{Z}_{\left[\left(\Phi_{\mathcal{H}}, \delta_{\mathcal{H}}, \sigma\right)\right]}$ admits the structure of a locally closed subscheme of $\mathrm{M}_{\mathcal{H}, \Sigma}^{\text {tor }}$ (with its reduced structure). 
Proof. The canonical morphism (9.9) exists by Proposition 5.24 (with the $\Sigma$ there given by the $\Sigma^{\prime}$ here) and by comparing the universal properties of $\mathrm{M}_{\mathcal{H}, \Sigma^{\prime}}^{\text {tor }}$ and $\mathrm{M}_{\mathcal{H}, \Sigma}^{\text {tor }}$ in [30, Theorem 6.4.1.1(6)] and Theorem 7.14, respectively, which is proper because $\mathrm{M}_{\mathcal{H}, \Sigma^{\prime}}^{\text {tor }}$ is proper over $\mathrm{S}_{0}$.

Suppose $t^{\prime}$ is any point of the subset $\mathrm{Z}_{\left[\left(\Phi_{\mathcal{H}}, \delta_{\mathcal{H}}, \tau\right)\right]}$ of $\mathrm{M}_{\mathcal{H}, \Sigma^{\prime}}^{\text {tor }}$, which is mapped to some point $t$ of $\mathrm{M}_{\mathcal{H}, \Sigma}^{\text {tor }}$. Suppose $\operatorname{Spec}(V) \rightarrow \mathrm{M}_{\mathcal{H}, \Sigma^{\prime}}^{\text {tor }}$ is any morphism, where $V$ is a complete discrete valuation ring with fraction field $K$, algebraically closed residue field $k$, and discrete valuation $v$, which maps the generic point $\operatorname{Spec}(K)$ to some maximal point of $\mathrm{M}_{\mathcal{H}}$ and maps the special point $\operatorname{Spec}(k)$ to $t^{\prime}$. Then its composition with (9.9) defines a morphism $\operatorname{Spec}(V) \rightarrow \mathrm{M}_{\mathcal{H}, \Sigma}^{\text {tor }}$ as in Definition 9.1, and it follows that $t$ lies on the subset $\mathrm{Z}_{\left[\left(\Phi_{\mathcal{H}}, \delta_{\mathcal{H}}, \sigma\right)\right]}$ of $\mathrm{M}_{\mathcal{H}, \Sigma}^{\text {tor }}$, by [30, Theorem 6.4.1.1 (5) and (6)] (and the property of the Mumford family $\left({ }^{\odot} G,{ }^{\circ} \lambda,{ }^{\circ} i,{ }^{\circ} \alpha_{\mathcal{H}}\right)$ carried by $\mathfrak{X}_{\Phi_{\mathcal{H}}, \delta_{\mathcal{H}}, \tau}$ for each representative $\left(\Phi_{\mathcal{H}}, \delta_{\mathcal{H}}, \tau\right)$ of $\left.\left[\left(\Phi_{\mathcal{H}}, \delta_{\mathcal{H}}, \tau\right)\right]\right)$.

On the other hand, suppose $t$ is a point of $Z_{\left[\left(\Phi_{\mathcal{H}}, \delta_{\mathcal{H}}, \sigma\right)\right]}$. By definition, there exist some morphism $\operatorname{Spec}(V) \rightarrow \mathrm{M}_{\mathcal{H}, \Sigma}^{\text {tor }}$ as in the statement of Definition 9.1, for some $\left(\mathrm{Z}_{\mathcal{H}}^{\ddagger}, \Phi_{\mathcal{H}}^{\ddagger}, \delta_{\mathcal{H}}^{\ddagger}\right)$ and some $\sigma^{\ddagger} \in \Sigma_{\Phi_{\mathcal{H}}^{\ddagger}}$ as in Theorem 7.14, where $\sigma^{\ddagger}$ is minimal among such choices, such that $\left[\left(\Phi_{\mathcal{H}}^{\ddagger}, \delta_{\mathcal{H}}^{\ddagger}, \sigma^{\ddagger}\right)\right]=\left[\left(\Phi_{\mathcal{H}}, \delta_{\mathcal{H}}, \sigma\right)\right]$. Since (9.9) is $\operatorname{proper}, \operatorname{Spec}(V) \rightarrow \mathrm{M}_{\mathcal{H}, \Sigma}^{\text {tor }}$ lifts to some morphism $\operatorname{Spec}(V) \rightarrow \mathrm{M}_{\mathcal{H}, \Sigma^{\prime}}^{\text {tor }}$. Since $\sigma^{\ddagger}$ is minimal among such choices, we have $v \circ B^{\ddagger} \in \sigma^{\ddagger} \subset \mathbf{P}_{\Phi_{\mathcal{H}}^{\ddagger}}^{+}$(not just in the closure ${\overline{\sigma^{\ddagger}}}^{\ddagger}$, and hence $v \circ B^{\ddagger} \in \tau^{\ddagger} \subset \mathbf{P}_{\Phi_{\mathcal{H}}^{\ddagger}}^{+}$for some $\tau^{\ddagger} \in \Sigma_{\Phi_{\mathcal{H}}^{\ddagger}}^{\prime}$. By [30, Theorem 6.4.1.1(6)] and its proof based on [30, Proposition 6.3.3.11], $\operatorname{Spec}(V) \rightarrow \mathrm{M}_{\mathcal{H}, \Sigma^{\prime}}^{\text {tor }}$

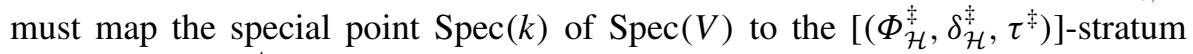
$\mathrm{Z}_{\left[\left(\Phi_{\mathcal{H}}^{\ddagger}, \delta_{\mathcal{H}}^{\ddagger}, \tau^{\ddagger}\right)\right]}$ of $\mathrm{M}_{\mathcal{H}, \Sigma^{\prime}}^{\text {tor }}$.

Thus we have shown that each $Z_{\left[\left(\Phi_{\mathcal{H}}, \delta_{\mathcal{H}}, \sigma\right)\right]}$ is the union of the images of all $Z_{\left[\left(\Phi_{\mathcal{H}}, \delta_{\mathcal{H}}, \tau\right)\right]}$ with $\tau \in \Sigma_{\Phi_{\mathcal{H}}}^{\prime} \in \Sigma^{\prime}$ contained in $\sigma \in \Sigma_{\Phi_{\mathcal{H}}} \in \Sigma$ in $\mathbf{P}_{\Phi_{\mathcal{H}}}^{+}$. The remaining assertions of the lemma then follow from this and from Lemma 9.3.

REMARK 9.10. The notation in Lemma 9.8 might be confusing, because $\mathrm{M}_{\mathcal{H}, \Sigma^{\prime}}^{\text {tor }}$ and $\mathrm{M}_{\mathcal{H}, \Sigma}^{\text {tor }}$ (and also $\mathbf{Z}_{\left[\left(\Phi_{\mathcal{H}}, \delta_{\mathcal{H}}, \tau\right)\right]}$ and $\mathrm{Z}_{\left[\left(\Phi_{\mathcal{H}}, \delta_{\mathcal{H}}, \sigma\right)\right]}$ ) are defined rather differently. This will be justified in Corollary 11.8 below.

LEMMA 9.11. For each $\left(\Phi_{\mathcal{H}}, \delta_{\mathcal{H}}, \sigma\right)$ (as in [30, Definition 6.2.6.1]) such that $\sigma \subset \mathbf{P}_{\Phi_{\mathcal{H}}}^{+}$and $\sigma \in \Sigma_{\Phi_{\mathcal{H}}} \in \Sigma$, the subset $\mathrm{Z}_{\left[\left(\Phi_{\mathcal{H}}, \delta_{\mathcal{H}}, \sigma\right)\right]}$ of $\vec{Z}_{\left[\left(\Phi_{\mathcal{H}}, \delta_{\mathcal{H}}, \sigma\right)\right]}$ is dense in $\overrightarrow{\mathrm{Z}}_{\left[\left(\Phi_{\mathcal{H}}, \delta \mathcal{H}, \sigma\right)\right]}$.

Proof. This follows from Lemma 9.4, because $\vec{\Xi}_{\Phi_{\mathcal{H}}, \delta_{\mathcal{H}}, \sigma}$ is smooth over $\vec{C}_{\Phi_{\mathcal{H}}, \delta_{\mathcal{H}}}$ and hence is flat over $\operatorname{Spec}\left(\mathbb{Z}_{(p)}\right)$ (see Lemma 8.20). 
LEMMA 9.12. For each $\mathrm{j} \in \mathrm{J}$, suppose that $\left(\mathrm{Z}_{\mathcal{H}_{\mathrm{j}, \text { aux }}}, \Phi_{\mathcal{H}_{\mathrm{j}, \text { aux }}}, \delta_{\mathcal{H}_{\mathrm{j}, \text { aux }}}\right)$ is induced by $\left(\mathrm{Z}_{\mathcal{H}}, \Phi_{\mathcal{H}}, \delta_{\mathcal{H}}\right)$ as in (5.13), and that $\sigma_{\mathrm{j} \text {,aux }} \in \Sigma_{\Phi_{\mathcal{H}_{\mathrm{j}, \text { aux }}}}$ and $\sigma_{\mathrm{j}, \text { aux }} \subset \mathbf{P}_{\Phi_{\mathcal{H}_{\mathrm{j}, \text { aux }}}^{+}}^{+}$, so that

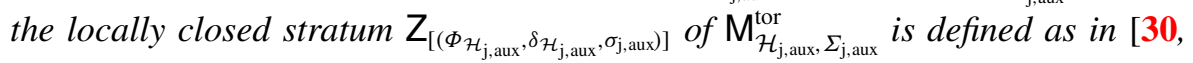
Theorem 6.4.1.1(2)]. Suppose $\sigma \in \Sigma_{\Phi_{\mathcal{H}}}$ is the pullback of $\prod_{\mathrm{j} \in \mathrm{J}} \sigma_{\mathrm{j} \text {,aux }}$ under (5.20), which lies in $\mathbf{P}_{\Phi_{\mathcal{H}}}^{+}$because $\sigma_{\mathrm{j}, \text { aux }} \subset \mathbf{P}_{\Phi_{\mathcal{H}} \text {,aux }}^{+}$for all $\mathrm{j} \in \mathrm{J}$. Then $\overrightarrow{\mathrm{Z}}_{\left[\left(\Phi_{\mathcal{H}}, \delta_{\mathcal{H}}, \sigma\right)\right]}$ is open and closed in the preimage of the locally closed stratum $\prod_{\mathrm{j} \in \mathrm{J}} \mathrm{Z}_{\left[\left(\Phi_{\mathcal{H}_{\mathrm{j}, \text { aux }}}, \delta_{\mathcal{H}_{\mathrm{j}} \text {,aux }}, \sigma_{\mathrm{j}, \text { aux }}\right)\right]}$ of $\prod_{\mathrm{j} \in \mathrm{J}} \mathrm{M}_{\mathcal{H}_{\mathrm{j}, \text { aux }}, \Sigma_{\mathrm{j}, \text { aux }}}^{\text {tor }}$ under the finite morphism (7.8). Consequently, $\overrightarrow{\mathrm{Z}}_{\left[\left(\Phi_{\mathcal{H}}, \delta_{\mathcal{H}}, \sigma\right)\right]}$ also admits the structure of a reduced locally closed subscheme of $\overrightarrow{\mathrm{M}}_{\mathcal{H}, \Sigma}^{\text {tor }}$.

Proof. By comparing the universal properties (as in Theorem 7.14 and Definition 9.1 for $\vec{Z}_{\left[\left(\Phi_{\mathcal{H}}, \delta_{\mathcal{H}}, \sigma\right)\right]}$, and as in [30, Theorem 6.4.1.1(6) and its proof based on Proposition 6.3.3.11] for $Z_{\left[\left(\Phi_{\mathcal{H}_{\mathrm{j}, \text { aux }},}, \mathcal{H}_{\mathcal{H}_{\mathrm{j}, \text { aux }}}, \sigma_{\mathrm{j}, \text { aux }}\right]\right.}$, for all $\left.\mathrm{j} \in \mathrm{J}\right)$, the canonical

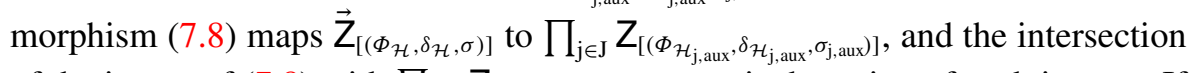

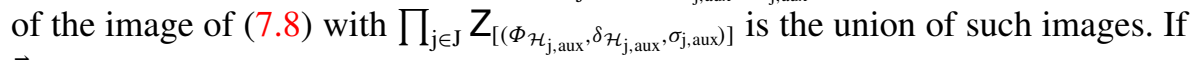
$\vec{Z}_{\left[\left(\Phi_{\mathcal{H}}^{\prime}, \delta_{\mathcal{H}}^{\prime}, \sigma^{\prime}\right)\right]}$ is also mapped to $\prod_{\mathrm{j} \in \mathrm{J}} \mathrm{Z}_{\left[\left(\Phi_{\mathcal{H}_{\mathrm{j}} \text {,aux }}, \delta_{\mathcal{H}_{\mathrm{j}} \text {,aux }}, \sigma_{\mathrm{j}, \text { aux }}\right]\right.}$, then the $\mathcal{O}$-multiranks of $\left[\left(\Phi_{\mathcal{H}}, \delta_{\mathcal{H}}\right)\right]$ and $\left[\left(\Phi_{\mathcal{H}}^{\prime}, \delta_{\mathcal{H}}^{\prime}\right)\right]$ have the same magnitude (see [30, Definitions 5.4.2.7 and 6.3.3.7]), because they induce the same $\left[\left(\Phi_{\mathcal{H}_{\mathrm{j}, \text { aux }}}, \delta_{\mathcal{H}_{\mathrm{j} \text {,aux }}}\right)\right]$ (for any $\mathrm{j} \in \mathrm{J}$ ), and hence none of $\left[\left(\Phi_{\mathcal{H}}, \delta_{\mathcal{H}}, \sigma\right)\right]$ and $\left[\left(\Phi_{\mathcal{H}}^{\prime}, \delta_{\mathcal{H}}^{\prime}, \sigma^{\prime}\right)\right]$ can be a face of the other (as in [30, Definition 6.3.2.14]), because $\sigma$ is assumed to be minimal among all choices in Definition 9.1. By Lemma 9.8, this shows that $Z_{\left[\left(\Phi_{\mathcal{H}}, \delta_{\mathcal{H}}, \sigma\right)\right]}$ is open and closed in

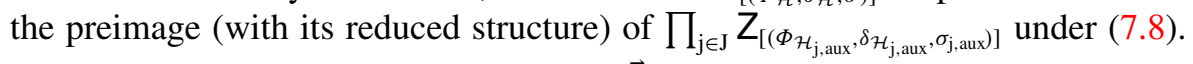
By Lemmas 9.3 and 9.11, it follows that $\vec{Z}_{\left[\left(\Phi_{\mathcal{H}}, \delta_{\mathcal{H}}, \sigma\right)\right]}$ is also open and closed in the preimage (with its reduced structure) of $\prod_{\mathrm{j} \in \mathrm{J}} Z_{\left[\left(\Phi_{\mathcal{H}_{\mathrm{j}, \text { aux }}}, \delta_{\mathcal{H}_{\mathrm{j}, \text { aux }}}, \sigma_{\mathrm{j}, \text { aux }}\right)\right]}$ under (7.8), as desired.

THEOREM 9.13 (Cf. [30, Theorem 6.4.1.1(2)]). With the setting as above, $\overrightarrow{\mathrm{M}}_{\mathcal{H}, \Sigma}^{\text {tor }}$ has a stratification by locally closed subschemes

$$
\overrightarrow{\mathrm{M}}_{\mathcal{H}, \Sigma}^{\text {tor }}=\coprod_{\left[\left(\Phi_{\mathcal{H}}, \delta_{\mathcal{H}}, \sigma\right)\right]} \overrightarrow{\mathbf{Z}}_{\left[\left(\Phi_{\mathcal{H}}, \delta_{\mathcal{H}}, \sigma\right)\right]}
$$

with $\left[\left(\Phi_{\mathcal{H}}, \delta_{\mathcal{H}}, \sigma\right)\right]$ running through a complete set of equivalence classes of $\left(\Phi_{\mathcal{H}}\right.$, $\left.\delta_{\mathcal{H}}, \sigma\right)$ (as in [30, Definition 6.2.6.1]) with $\sigma \subset \mathbf{P}_{\Phi_{\mathcal{H}}}^{+}$and $\sigma \in \Sigma_{\Phi_{\mathcal{H}}} \in \Sigma$, where each stratum $\vec{Z}_{\left[\left(\Phi_{\mathcal{H}}, \delta_{\mathcal{H}}, \sigma\right)\right]}$ is as in Definition 9.1 and Lemma 9.12 (here the notation ' ' only means a set-theoretic disjoint union. The algebro-geometric structure is still that of $\left.\overrightarrow{\mathrm{M}}_{\mathcal{H}, \Sigma}^{\mathrm{tor}}\right)$. In this stratification, the $\left[\left(\Phi_{\mathcal{H}}^{\prime}, \delta_{\mathcal{H}}^{\prime}, \sigma^{\prime}\right)\right]$-stratum $\overrightarrow{\mathrm{Z}}_{\left[\left(\Phi_{\mathcal{H}}^{\prime}, \delta_{\mathcal{H}}^{\prime}, \sigma^{\prime}\right)\right]}$ lies in the closure of the $\left[\left(\Phi_{\mathcal{H}}, \delta_{\mathcal{H}}, \sigma\right)\right]$-stratum $\vec{Z}_{\left[\left(\Phi_{\mathcal{H}}, \delta_{\mathcal{H}}, \sigma\right)\right]}$ if and only if 
$\left[\left(\Phi_{\mathcal{H}}, \delta_{\mathcal{H}}, \sigma\right)\right]$ is a face of $\left[\left(\Phi_{\mathcal{H}}^{\prime}, \delta_{\mathcal{H}}^{\prime}, \sigma^{\prime}\right)\right]$ as in [30, Definition 6.3.2.14] (see also [30, Remark 6.3.2.15]). The open dense subscheme $\overrightarrow{\mathrm{M}}_{\mathcal{H}}$ of $\overrightarrow{\mathrm{M}}_{\mathcal{H}, \Sigma}^{\text {tor }}$ (see Lemma 7.9) coincides with the $[(0,0,\{0\})]$-stratum $\vec{Z}_{[(0,0,\{0\})]}$ in this stratification.

Proof. By Lemma 9.3, $\overrightarrow{\mathrm{M}}_{\mathcal{H}, \Sigma}^{\text {tor }}=\bigcup_{\left[\left(\Phi_{\mathcal{H}}, \delta_{\mathcal{H}}, \sigma\right)\right]} \overrightarrow{\mathrm{Z}}_{\left[\left(\Phi_{\mathcal{H}}, \delta_{\mathcal{H}}, \sigma\right)\right]}$, with $\left[\left(\Phi_{\mathcal{H}}, \delta_{\mathcal{H}}, \sigma\right)\right]$ running through all equivalence classes, with $\sigma \subset \mathbf{P}_{\Phi_{\mathcal{H}}}^{+}$and $\sigma \in \Sigma_{\Phi_{\mathcal{H}}} \in \Sigma$. By definition, the assignment of $\left[\left(\Phi_{\mathcal{H}_{\mathrm{j}, \text { aux }}}, \delta_{\mathcal{H}_{\mathrm{j}, \text { aux }}}, \sigma_{\mathrm{j} \text {,aux }}\right)\right]$ to $\left[\left(\Phi_{\mathcal{H}}, \delta_{\mathcal{H}}, \sigma\right)\right]$ as in Definition 5.23 respects the incidence relations as in [30, Definition 6.3.2.13], for each $\mathrm{j} \in \mathrm{J}$. Therefore, by Lemmas 9.11 and 9.12, in order to show that the above union defines a stratification of $\overrightarrow{\mathrm{M}}_{\mathcal{H}, \Sigma}^{\text {tor }}$ with the desired incidence relation described as in the second paragraph of this proposition, it suffices to note that, by Lemma 9.8, its pullback to $\mathbf{M}_{\mathcal{H}, \Sigma}^{\text {tor }}=\vec{M}_{\mathcal{H}, \Sigma}^{\text {tor }} \otimes_{\mathbb{Z}} \mathbb{Q}$ does. Similarly, in order to show that the subscheme $\vec{Z}_{[(0,0,\{0\})]}$ of $\vec{M}_{\mathcal{H}, \Sigma}^{\text {tor }}$ coincides with $\vec{M}_{\mathcal{H}}$, it suffices to note that, by Lemma 9.8, the subscheme $Z_{[(0,0,\{0\})]}=\vec{Z}_{[(0,0,\{0\})]} \otimes_{\mathbb{Z}} \mathbb{Q}$ of $M_{\mathcal{H}, \Sigma}^{\text {tor }}=\vec{M}_{\mathcal{H}, \Sigma}^{\text {tor }} \otimes_{\mathbb{Z}} \mathbb{Q}$ coincides with $M_{\mathcal{H}} \cong \vec{M}_{\mathcal{H}} \otimes_{\mathbb{Z}} \mathbb{Q}$.

REMARK 9.14. In Theorems 12.1 and 12.16 below, we will see that the corresponding analogs of [30, Theorem 7.2.4.1 (4) and (5)] are also true for $\overrightarrow{\mathrm{M}}_{\mathcal{H}}^{\min }$.

\section{Comparison of formal completions}

The main goal of this section is to show that an analog of [30, Theorem 6.4.1.1(5)] is true for $\vec{M}_{\mathcal{H}, \Sigma}^{\text {tor }}$ (see Theorem 10.13 below).

Let $\vec{Z}_{\left[\left(\Phi_{\mathcal{H}}, \delta_{\mathcal{H}}, \sigma\right)\right]}$ be the $\left[\left(\Phi_{\mathcal{H}}, \delta_{\mathcal{H}}, \sigma\right)\right]$-stratum of $\overrightarrow{\mathrm{M}}_{\mathcal{H}, \Sigma}^{\text {tor }}$ as in Theorem 9.13.

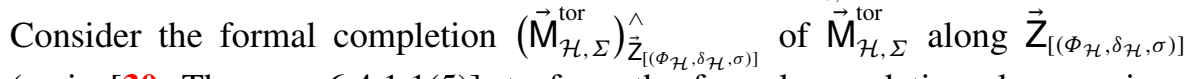
(as in [30, Theorem 6.4.1.1(5)], to form the formal completion along a given locally closed stratum, we first remove the other strata appearing in the closure of this stratum from the total space, and then form the formal completion of the remaining space along this stratum). Then the canonical finite morphism (7.8) of schemes induces a canonical finite morphism

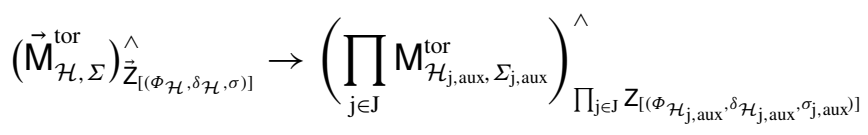

of formal schemes. 
By [30, Theorem 6.4.1.1(5)], the morphism (8.31) induces a canonical isomorphism

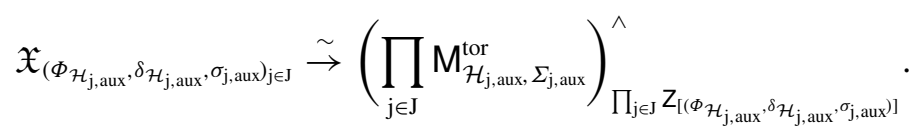

By Lemma 9.4, the morphism (8.33) induces a morphism

$$
\overrightarrow{\mathfrak{X}}_{\Phi_{\mathcal{H}}, \delta \mathcal{H}, \sigma} \rightarrow\left(\overrightarrow{\mathrm{M}}_{\mathcal{H}, \Sigma}^{\text {tor }}\right)_{\hat{\mathrm{Z}}_{\left[\left(\Phi_{\mathcal{H}}, \delta \mathcal{H}, \sigma\right)\right]}}^{\wedge},
$$

which is compatible with (8.21) and (10.1) by construction. The pullback of (10.3) to characteristic zero defines a morphism

$$
\mathfrak{X}_{\Phi_{\mathcal{H}}, \delta_{\mathcal{H}}, \sigma} \rightarrow\left(\mathrm{M}_{\mathcal{H}, \Sigma}^{\text {tor }}\right)_{\mathrm{Z}_{\left[\left(\Phi_{\mathcal{H}}, \delta \mathcal{H}, \sigma\right)\right]}}^{\wedge} .
$$

Let $\Sigma^{\prime}$ be as in Lemma 9.8, so that we have the canonical proper surjective morphism $\mathrm{M}_{\mathcal{H}, \Sigma^{\prime}}^{\text {tor }} \rightarrow \mathrm{M}_{\mathcal{H}, \Sigma}^{\text {tor }}$ as in (9.9). Consider

$$
\Xi_{\Phi_{\mathcal{H}}, \delta \mathcal{H}}(\sigma)^{\prime}:=\bigcup_{\tau \in \Sigma_{\Phi_{\mathcal{H}}}^{\prime}, \tau \subset \sigma} \Xi_{\Phi_{\mathcal{H}}, \delta \mathcal{H}}(\tau),
$$

the toroidal embedding of $\Xi_{\Phi_{\mathcal{H}}, \delta_{\mathcal{H}}}$ defined by gluing the affine toroidal embeddings $\Xi_{\Phi_{\mathcal{H}}, \delta_{\mathcal{H}}}(\tau)$ as in [30, Section 6.2.5], with $\tau$ running through the cones in $\Sigma_{\Phi_{\mathcal{H}}}^{\prime}$ that are contained in $\sigma$. Then there is a canonical proper morphism

$$
\Xi_{\Phi_{\mathcal{H}}, \delta_{\mathcal{H}}}(\sigma)^{\prime} \rightarrow \Xi_{\Phi_{\mathcal{H}}, \delta_{\mathcal{H}}}(\sigma)
$$

(cf. [24, Ch. I, Section 2, Theorem 8]), which induces the identity morphism on $\Xi_{\Phi_{\mathcal{H}}, \delta_{\mathcal{H}}}$ by restriction. The preimage of the closed $\sigma$-stratum $\Xi_{\Phi_{\mathcal{H}}, \delta_{\mathcal{H}}, \sigma}$ of $\Xi_{\Phi_{\mathcal{H}}, \delta_{\mathcal{H}}}(\sigma)$ under this proper morphism (with its reduced structure) is the union

$$
\Xi_{\Phi_{\mathcal{H}}, \delta_{\mathcal{H}}, \sigma}^{\prime}:=\bigcup_{\tau \in \Sigma_{\Phi_{\mathcal{H}}}^{\prime}, \tau \subset \sigma} \Xi_{\Phi_{\mathcal{H}}, \delta_{\mathcal{H}}, \tau} .
$$

Let $\mathfrak{X}_{\Phi_{\mathcal{H}}, \delta_{\mathcal{H}}, \sigma}^{\prime}$ denote the formal completion of $\Xi_{\Phi_{\mathcal{H}}, \delta_{\mathcal{H}}}(\sigma)^{\prime}$ along its closed subscheme $\Xi_{\Phi_{\mathcal{H}}, \delta_{\mathcal{H}}, \sigma}^{\prime}$. Then (10.6) induces a proper morphism

$$
\mathfrak{X}_{\Phi_{\mathcal{H}}, \delta \mathcal{H}, \sigma}^{\prime} \rightarrow \mathfrak{X}_{\Phi_{\mathcal{H}}, \delta_{\mathcal{H}}, \sigma}
$$

By the same argument as in [30, Section 6.2.5], we also have a Mumford family

$$
\left({ }^{\odot} G,{ }^{\circ} \lambda,{ }^{\odot} i,{ }^{\odot} \alpha_{\mathcal{H}}\right) \rightarrow \mathfrak{X}_{\Phi_{\mathcal{H}}, \delta_{\mathcal{H}}, \sigma}^{\prime},
$$


which is canonically isomorphic to the pullback of the above Mumford family (8.28) under the morphism (10.8). Let us denote by $Z_{\left[\left(\Phi_{\mathcal{H}}, \delta_{\mathcal{H}}, \sigma\right)\right]}^{\prime}$ the preimage (with its reduced structure) of $Z_{\left[\left(\Phi_{\mathcal{H}}, \delta_{\mathcal{H}}, \sigma\right)\right]}$ under (9.9). By Lemma 9.8, $\mathbf{Z}_{\left[\left(\Phi_{\mathcal{H}}, \delta_{\mathcal{H}}, \sigma\right)\right]}^{\prime}$ is the union of $Z_{\left[\left(\Phi_{\mathcal{H}}, \delta_{\mathcal{H}}, \tau\right)\right]}$ with the same $\tau$ 's as in (10.5). Then (9.9) induces a proper morphism

$$
\left(\mathrm{M}_{\mathcal{H}, \Sigma^{\prime}}^{\text {tor }}\right)_{\mathrm{Z}_{\left[\left(\Phi_{\mathcal{H}}, \delta \mathcal{H}, \sigma\right)\right]}^{\prime}}^{\wedge} \rightarrow\left(\mathrm{M}_{\mathcal{H}, \Sigma}^{\text {tor }}\right)_{\mathrm{Z}_{\left.\left[\Phi_{\mathcal{H}}, \delta_{\mathcal{H}}, \sigma\right)\right]}^{\wedge}}^{\wedge}
$$

By the same argument as in the proofs of [30, Theorem 6.4.1.1(5)] and [29, Proposition 4.3], which are based on [30, Theorem 6.4.1.1(6)], there is a canonical isomorphism

$$
\mathfrak{X}_{\Phi_{\mathcal{H}}, \delta \mathcal{H}, \sigma}^{\prime} \stackrel{\sim}{\rightarrow}\left(\mathrm{M}_{\mathcal{H}, \Sigma^{\prime}}^{\mathrm{tor}}\right)_{\mathrm{Z}_{\left[\left(\Phi_{\mathcal{H}}, \delta_{\mathcal{H}}, \sigma\right)\right]}^{\prime}}^{\wedge}
$$

such that the Mumford family (10.9) is the pullback of the tautological degenerating family $\left(G, \lambda, i, \alpha_{\mathcal{H}}\right)$ over $\mathrm{M}_{\mathcal{H}, \Sigma^{\prime}}^{\text {tor }}$ under the composition of (10.11) with the canonical morphism $\left(\mathrm{M}_{\mathcal{H}, \Sigma^{\prime}}^{\text {tor }}\right)_{Z_{\left[\left(\Phi_{\mathcal{H}}, \delta_{\mathcal{H}}, \sigma\right]\right]}^{\prime}}^{\wedge} \rightarrow \mathrm{M}_{\mathcal{H}, \Sigma^{\prime}}^{\text {tor }}$. Hence we have a commutative diagram

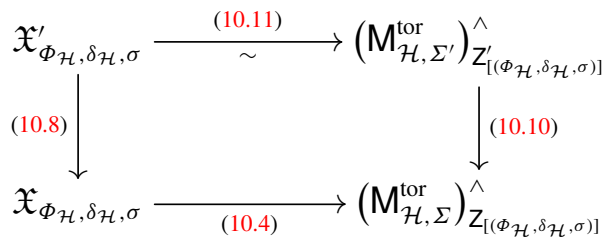

by the universal properties of the objects involved.

THEOREM 10.13 (Cf. [30, Theorem 6.4.1.1(5)]). With the setting as above, the morphism (10.3) is an isomorphism.

Proof. Since (8.21) and (10.1) are finite, and since (10.2) is an isomorphism, (10.3) is also finite; that is, under (10.3), the preimage of each affine open formal subscheme $\operatorname{Spf}(R, I)$ of $\left(\overrightarrow{\mathrm{M}}_{\mathcal{H}, \Sigma}^{\text {tor }}\right)_{\hat{\mathbf{Z}}_{\left[\left(\Phi_{\mathcal{H}}, \delta_{\mathcal{H}}, \sigma\right)\right]}}$ in $\overrightarrow{\mathfrak{X}}_{\Phi_{\mathcal{H}}, \delta_{\mathcal{H}}, \sigma}$ is isomorphic to $\operatorname{Spf}(\tilde{R}$, $\tilde{I}$ ) for some finite $R$-algebra $\tilde{R}$ and for $\tilde{I}:=I \cdot \tilde{R} \subset \tilde{R}$ (cf. [14, III-1, 5.1.4]). In order to show that (10.3) is an isomorphism, it suffices to show that, over each such $\operatorname{Spf}(R, I)$, the finite morphism $\operatorname{Spec}(\tilde{R}) \rightarrow \operatorname{Spec}(R)$ induced by (10.3) is an isomorphism. Since $\overrightarrow{\mathrm{M}}_{\mathcal{H}, \Sigma}^{\text {tor }}$ and $\vec{\Xi}_{\Phi_{\mathcal{H}}, \delta_{\mathcal{H}}}(\sigma)$ are flat over $\overrightarrow{\mathrm{S}}_{0}$ and excellent normal, $R$ and $\tilde{R}$ are flat over $\mathbb{Z}_{(p)}$ and noetherian normal. Hence, by Zariski's 
main theorem (see [14, III-1, 4.4.3, 4.4.11]), it suffices to show that the induced finite homomorphism

$$
R \underset{\mathbb{Z}}{\otimes} \mathbb{Q} \rightarrow \underset{\mathbb{Z}}{\otimes} \mathbb{Q}
$$

is an isomorphism.

Let $\mathfrak{U}:=\operatorname{Spf}\left(R \otimes_{\mathbb{Z}} \mathbb{Q}, I \otimes_{\mathbb{Z}} \mathbb{Q}\right)$ and $\tilde{\mathfrak{U}}:=\operatorname{Spf}\left(\tilde{R} \otimes_{\mathbb{Z}} \mathbb{Q}, \tilde{I} \otimes_{\mathbb{Z}} \mathbb{Q}\right)$, the latter being the preimage of the former under the (finite) morphism (10.4), so that (10.14) can be identified with the canonical homomorphism $\Gamma\left(\mathfrak{U}, \mathscr{O}_{\mathfrak{U}}\right) \rightarrow \Gamma(\tilde{\mathfrak{U}}$, $\left.\mathscr{O}_{\tilde{\mathfrak{U}}}\right)$. Let $\mathfrak{U}^{\prime}$ (respectively $\tilde{\mathfrak{U}}^{\prime}$ ) denote the preimage of $\mathfrak{U}$ (respectively $\tilde{\mathfrak{U}}$ ) under (10.10) (respectively (10.8)), with the induced structure of an open formal subscheme of $\left(\mathrm{M}_{\mathcal{H}, \Sigma^{\prime}}^{\text {tor }}\right)_{Z_{\left[\left(\Phi_{\mathcal{H}}, \delta \mathcal{H}, \sigma\right)\right]}^{\prime}}^{\wedge}$ (respectively $\left.\mathfrak{X}_{\Phi_{\mathcal{H}}, \delta \mathcal{H}, \sigma}^{\prime}\right)$. By [14, III-1, 4.1.5] and by Zariski's main theorem (see [14, III-1, 4.4.3, 4.4.11]), since the proper morphism (9.9) induces by restriction the identity morphism on the open dense subscheme $\mathrm{M}_{\mathcal{H}}$, and since $\mathrm{M}_{\mathcal{H}, \Sigma}^{\text {tor }}$ is noetherian and normal by construction, the canonical morphism $\Gamma\left(\mathfrak{U}, \mathscr{O}_{\mathfrak{U}}\right) \rightarrow \Gamma\left(\mathfrak{U}^{\prime}, \mathscr{O}_{\mathfrak{U}^{\prime}}\right)$ is an isomorphism. Similarly, since the proper morphism (10.6) induces by restriction the identity morphism on the open dense subscheme $\Xi_{\Phi_{\mathcal{H}}, \delta_{\mathcal{H}}}$, and since $\Xi_{\Phi_{\mathcal{H}}, \delta_{\mathcal{H}}}(\sigma)$ is noetherian and normal by construction, the canonical morphism $\Gamma\left(\tilde{\mathfrak{U}}, \mathscr{O}_{\tilde{\mathfrak{U}}}\right) \rightarrow \Gamma\left(\tilde{\mathfrak{U}}^{\prime}, \mathscr{O}_{\tilde{\mathfrak{U}}^{\prime}}\right)$ is an isomorphism. Since the diagram (10.12) is commutative, the morphism (10.11) induces an isomorphism $\tilde{\mathfrak{U}}^{\prime} \stackrel{\sim}{\rightarrow} \mathfrak{U}^{\prime}$. Combining all of these, the canonical homomorphism $\Gamma\left(\mathfrak{U}, \mathscr{O}_{\mathfrak{U}}\right) \rightarrow \Gamma\left(\tilde{\mathfrak{U}}, \mathscr{O}_{\mathfrak{U}}\right)$ can be identified with the canonical isomorphism $\Gamma\left(\mathfrak{U}^{\prime}, \mathscr{O}_{\mathfrak{U}^{\prime}}\right) \stackrel{\sim}{\rightarrow} \Gamma\left(\tilde{\mathfrak{U}}^{\prime}, \mathscr{O}_{\tilde{U}^{\prime}}\right)$, and it follows that (10.14) is an isomorphism, as desired.

COROLlaRY 10.15. With the setting as above, $\vec{Z}_{\left[\left(\Phi_{\mathcal{H}}, \delta_{\mathcal{H}}, \sigma\right)\right]}$ is canonically isomorphic to the scheme $\vec{\Xi}_{\Phi_{\mathcal{H}}, \delta_{\mathcal{H}}, \sigma}$ in Lemma 8.20, which is an $E_{\Phi_{\mathcal{H}}, \sigma}$-torsor over the scheme $\vec{C}_{\Phi_{\mathcal{H}}, \delta_{\mathcal{H}}}$ proper over $\overrightarrow{\mathrm{M}}_{\mathcal{H}}^{Z_{\mathcal{H}}}$ (see Propositions 8.1 and 8.4). Consequently, $\vec{Z}_{\left[\left(\Phi_{\mathcal{H}}, \delta_{\mathcal{H}}, \sigma\right)\right]}$ is smooth over $\vec{C}_{\Phi_{\mathcal{H}}, \delta_{\mathcal{H}}}$, and hence it is flat over $\overrightarrow{\mathrm{S}}_{0}$ and normal because $\vec{C}_{\Phi_{\mathcal{H}}, \delta_{\mathcal{H}}}$ is. Moreover, $\overrightarrow{\mathrm{Z}}_{\left[\left(\Phi_{\mathcal{H}}, \delta_{\mathcal{H}}, \sigma\right)\right]}$ is proper over $\overrightarrow{\mathrm{M}}_{\mathcal{H}}^{\mathrm{ZH}}$ if $\sigma$ is top-dimensional in $\mathbf{P}_{\Phi_{\mathcal{H}}}^{+} \subset\left(\mathbf{S}_{\Phi_{\mathcal{H}}}\right)_{\mathbb{R}}^{\vee}$, in which case $\sigma^{\perp}=\{0\}$ and the torus $E_{\Phi_{\mathcal{H}}, \sigma}$ is trivial.

Proof. The first assertion follows from Theorem 10.13, because the canonical isomorphism (10.3) between formal schemes necessarily induces a canonical isomorphism $\vec{\Xi}_{\Phi_{\mathcal{H}}, \delta_{\mathcal{H}}, \sigma} \stackrel{\sim}{\rightarrow} \vec{Z}_{\left[\left(\Phi_{\mathcal{H}}, \delta_{\mathcal{H}}, \sigma\right)\right]}$ between the supporting schemes (with their reduced structures). The remaining assertions are self-explanatory.

Corollary 10.16. For each $\mathrm{j} \in \mathrm{J}$, let $\left(\vec{G}_{\mathrm{j}, \text { aux }}, \vec{\lambda}_{\mathrm{j} \text {,aux }}, \overrightarrow{ }_{\mathrm{j}, \text { aux }}, \vec{\alpha}_{\mathcal{H}_{\mathrm{j}, \text { aux }}}\right) \rightarrow \overrightarrow{\mathrm{M}}_{\mathcal{H}, \Sigma}^{\text {tor }}$ denote the pullback of the tautological tuple $\left(G_{\mathrm{j}, \text { aux }}, \lambda_{\mathrm{j}, \text { aux }}, i_{\mathrm{j}, \text { aux }}, \alpha_{\mathcal{H}_{\mathrm{j}, \text { aux }}}\right)$ over $\mathrm{M}_{\mathcal{H}_{\mathrm{j}, \text { aux }}, \Sigma_{\mathrm{j} \text {,aux }}}^{\text {tor }}$ 


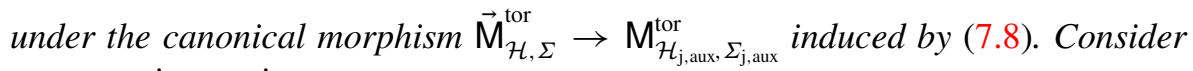
any open immersion

$$
\operatorname{Spf}(R, I) \rightarrow\left(\overrightarrow{\mathrm{M}}_{\mathcal{H}, \Sigma}^{\text {tor }}\right)_{\left.\left.\hat{\mathrm{Z}}_{\left[\left(\Phi_{\mathcal{H}}, \delta \mathcal{H}\right.\right.}, \sigma\right)\right]},
$$

where $R$ is a noetherian domain which is complete with respect to some ideal I. Then the preimage of $\overrightarrow{\mathrm{M}}_{\mathcal{H}}$ under the canonical morphism $\operatorname{Spec}(R) \rightarrow \overrightarrow{\mathrm{M}}_{\mathcal{H}, \Sigma}^{\text {tor }}$ induced by (10.17) is the maximal open subscheme of $\operatorname{Spec}(R)$ over which the pullback of $\vec{G}_{\mathrm{j}, \text { aux }}$ is an abelian scheme for at least one (and hence for every) $\mathrm{j} \in \mathrm{J}$, which coincides with the preimage of $\Xi_{\Phi_{\mathcal{H}}, \delta_{\mathcal{H}}}$ under the canonical morphism $\operatorname{Spec}(R) \rightarrow \vec{\Xi}_{\Phi_{\mathcal{H}}, \delta_{\mathcal{H}}}(\sigma)$ induced by the composition of (10.17) with the inverse of (10.3).

Proof. This is because (10.3) is defined by the universal property of $\overrightarrow{\mathrm{M}}_{\mathcal{H}, \Sigma}^{\text {tor }}$ as in Theorem 7.14, using the Mumford families (8.27), for all $\mathrm{j} \in \mathrm{J}$; because a fiber product of semiabelian schemes is isogenous to an abelian scheme exactly when all the factors are abelian schemes; and because the Mumford families (8.29) (whose self-fiber products induce (8.27) by isogeny) are abelian schemes exactly over the preimage of $\vec{\Xi}_{\Phi_{\mathcal{H}}, \delta \mathcal{H}}$, by the last assertion in Proposition 8.14.

Corollary 10.18. $\overrightarrow{\mathrm{M}}_{\mathcal{H}} \otimes_{\mathbb{Z}} \mathbb{F}_{p}$ is dense in $\overrightarrow{\mathrm{M}}_{\mathcal{H}, \Sigma}^{\text {tor }} \otimes_{\mathbb{Z}} \mathbb{F}_{p}$.

Proof. This follows from Proposition 8.14; Theorems 9.13 and 10.13; and Corollary 10.16.

\section{Semiabelian extensions of tautological objects}

The main goal of this section is to show that, for each $\mathrm{j} \in \mathrm{J}$, the degenerating family of type $M_{\mathcal{H}_{j}}$ over $\vec{M}_{\mathcal{H}}$ in Proposition 6.1 further extends to a degenerating family of the same type over $\overrightarrow{\mathrm{M}}_{\mathcal{H}, \Sigma}^{\text {tor }}$ (see Theorem 11.2 below). As a byproduct, we will also improve Theorem 7.14 (see Theorem 11.4 below), and deduce from this that, up to canonical isomorphism, the scheme $\overrightarrow{\mathrm{M}}_{\mathcal{H}, \Sigma}^{\text {tor }}$ constructed in Section 7 is independent of the auxiliary choices in Sections 4 and 5 (see Corollary 11.7 below).

LeMma 11.1. Suppose $\left(\Phi_{\mathcal{H}}, \delta_{\mathcal{H}}, \sigma\right)$, where $\sigma \subset \mathbf{P}_{\Phi_{\mathcal{H}}}^{+}$and $\sigma \in \Sigma_{\Phi_{\mathcal{H}}}$, is as in [30, Definition 6.2.6.1], and suppose $\left[\left(\Phi_{\mathcal{H}}, \delta_{\mathcal{H}}, \sigma\right)\right] \neq[(0,0,\{0\})]$. Let $U$ be any open subscheme of $\overrightarrow{\mathrm{M}}_{\mathcal{H}, \Sigma}^{\text {tor }}$ that is a union of strata and contains $\vec{Z}_{\left[\left(\Phi_{\mathcal{H}}, \delta_{\mathcal{H}}, \sigma\right)\right]}$ as a closed subscheme; and let $U^{\prime}$ be the complement of $\vec{Z}_{\left[\left(\Phi_{\mathcal{H}}, \delta_{\mathcal{H}}, \sigma\right)\right]}$ in $U$, which 
necessarily contains $\vec{Z}_{[(0,0,\{0\})]}=\vec{M}_{\mathcal{H}}$ because $\left[\left(\Phi_{\mathcal{H}}, \delta_{\mathcal{H}}, \sigma\right)\right] \neq[(0,0,\{0\})]($ see Theorem 9.13). By definition, the formal completion $\mathfrak{U}$ of $U$ along $\vec{Z}_{\left[\left(\Phi_{\mathcal{H}}, \delta_{\mathcal{H}}, \sigma\right)\right]}$ can be canonically identified with $\left(\overrightarrow{\mathrm{M}}_{\mathcal{H}, \Sigma}^{\text {tor }}\right)_{\overrightarrow{\mathrm{Z}}_{\left[\left(\Phi_{\mathcal{H}}, \delta_{\mathcal{H}}, \sigma\right)\right]}}$. By Theorem 10.13 , we have a canonical isomorphism $\overrightarrow{\mathfrak{X}}_{\Phi_{\mathcal{H}}, \mathcal{H}_{\mathcal{H}}, \sigma} \stackrel{\sim}{\rightarrow} \mathfrak{U}$ given by (10.3). Suppose $\mathrm{j} \in \mathrm{J}$, and suppose the tautological object $\left(A_{\mathrm{j}}, \lambda_{\mathrm{j}}, i_{\mathrm{j}}, \alpha_{\mathcal{H}_{\mathrm{j}}}\right)$ over $\mathrm{M}_{\mathcal{H}_{\mathrm{j}}} \cong \mathrm{M}_{\mathcal{H}}$ (see (2.1)) extends to a degenerating family $\left(G_{\mathrm{j}, U^{\prime}}, \lambda_{\mathrm{j}, U^{\prime}}, i_{\mathrm{j}, U^{\prime}}, \alpha_{\mathcal{H}_{\mathrm{j}}, U^{\prime}}\right)$ of type $\mathrm{M}_{\mathcal{H}_{\mathrm{j}}}$ over $U^{\prime}$ (see [30, Definition 5.3.2.1]), where $\alpha_{\mathcal{H}_{\mathrm{j}}, U^{\prime}}$ is only required to be defined over $\mathrm{M}_{\mathcal{H}}$. Then this degenerating family further extends to a degenerating family $\left(G_{\mathrm{j}, U}, \lambda_{\mathrm{j}, U}\right.$, $\left.i_{\mathrm{j}, U}, \alpha_{\mathcal{H}_{\mathrm{j}}, U}\right)$ of type $\mathrm{M}_{\mathcal{H}_{\mathrm{j}}}$ over $U$.

Proof. By the construction of (10.3), and by the construction of the morphisms involved in the commutative diagram (10.12), for each affine open subscheme $\operatorname{Spec}(R)$ of $U$ inducing an affine open subscheme $\operatorname{Spf}\left(R^{\wedge}, I\right)$ of $\overrightarrow{\mathfrak{X}}_{\Phi_{\mathcal{H}}, \delta_{\mathcal{H}}, \sigma} \cong \mathfrak{U}$, with canonical morphisms $\operatorname{Spec}\left(R^{\wedge}\right) \rightarrow \operatorname{Spec}(R) \rightarrow U$, there is a canonical isomorphism over the preimage of $\mathrm{M}_{\mathcal{H}}$ in $\operatorname{Spec}\left(R^{\wedge}\right)$ between the pullbacks of the tautological object $\left(A_{\mathrm{j}}, \lambda_{\mathrm{j}}, i_{\mathrm{j}}, \alpha_{\mathcal{H}_{\mathrm{j}}}\right)$ over $\mathrm{M}_{\mathcal{H}_{\mathrm{j}}} \cong \mathrm{M}_{\mathcal{H}}$ and the Mumford family $\left({ }^{\odot} \vec{G}_{\mathrm{j}},{ }^{\circ} \vec{\lambda}_{\mathrm{j}},{ }^{\circ} \vec{i}_{\mathrm{j}},{ }^{\circ} \vec{\alpha}_{\mathcal{H}_{\mathrm{j}}}\right.$ ) over $\overrightarrow{\mathfrak{X}}_{\Phi_{\mathcal{H}}, \delta_{\mathcal{H}}, \sigma}$ (see (8.29)). Since $\overrightarrow{\mathrm{M}}_{\mathcal{H}, \Sigma}^{\text {tor }}$ is excellent normal, both $R$ and $R^{\wedge}$ are noetherian normal (see [14, IV-2, 7.8.3.1]). By [52, IX, 1.4], [10, Ch. I, Proposition 2.7], or [30, Proposition 3.3.1.5], the above canonical isomorphism uniquely extends to a canonical isomorphism over the preimage of $U^{\prime}$ in $\operatorname{Spec}\left(R^{\wedge}\right)$ between the pullbacks of $\left(G_{\mathrm{j}, U^{\prime}}, \lambda_{\mathrm{j}, U^{\prime}}, i_{\mathrm{j}, U^{\prime}}, \alpha_{\mathcal{H}_{\mathrm{j}}, U^{\prime}}\right)$ and $\left({ }^{\odot} \vec{G}_{\mathrm{j}},{ }^{\odot} \vec{\lambda}_{\mathrm{j}},{ }^{\odot} \vec{i}_{\mathrm{j}},{ }^{\odot} \vec{\alpha}_{\mathcal{H}_{\mathrm{j}}}\right)$, which induces a canonical isomorphism between the pullbacks of the relative ample $\left(\operatorname{Id}_{G_{\mathrm{j}, U^{\prime}}}, \lambda_{\mathrm{j}, U^{\prime}}\right)^{*} \mathcal{P}_{G_{\mathrm{j}, U^{\prime}}}$ and $\left(\operatorname{Id}_{\odot} \vec{G}_{\mathrm{j}},{ }^{\circ} \vec{\lambda}_{\mathrm{j}}\right) * \mathcal{P}_{\odot} \vec{G}_{\mathrm{j}}$ (see [52, XI, 1.13] and [30, Theorem 3.4.3.2, Proposition 3.3.2.2, and Theorem 3.3.2.3]). Therefore, by the theory of fpqc descent (see [13, VIII, 7.8] and [40, Theorem 1.1]), the pullback of $\left(G_{\mathrm{j}, U^{\prime}}, \lambda_{\mathrm{j}, U^{\prime}}, i_{\mathrm{j}, U^{\prime}}, \alpha_{\mathcal{H}_{\mathrm{j}}, U^{\prime}}\right)$ to the preimage of $U^{\prime}$ in $\operatorname{Spec}(R)$ extends to a degenerating family of type $\mathrm{M}_{\mathcal{H}_{\mathrm{j}}}$ over $\operatorname{Spec}(R)$, whose pullback to $\operatorname{Spec}\left(R^{\wedge}\right)$ is canonically isomorphic to the pullback of $\left({ }^{\odot} \vec{G}_{\mathrm{j}},{ }^{\odot} \vec{\lambda}_{\mathrm{j}}\right.$, $\left.{ }^{\odot} \vec{i}_{\mathrm{j}},{ }^{\odot} \vec{\alpha}_{\mathcal{H}_{\mathrm{j}}}\right)$. Since such extensions over affine open subschemes of $U$ are unique up to canonical isomorphism, they are compatible with each other and define a degenerating family $\left(G_{\mathrm{j}, U}, \lambda_{\mathrm{j}, U}, i_{\mathrm{j}, U}, \alpha_{\mathcal{H}_{\mathrm{j}}, U}\right)$ of $\mathrm{M}_{\mathcal{H}_{\mathrm{j}}}$ over $U$, as in the statement of the lemma.

THEOREM 11.2. For each $\mathrm{j} \in \mathrm{J}$, there is a degenerating family $\left(\vec{G}_{\mathrm{j}}, \vec{\lambda}_{\mathrm{j}}, \vec{i}_{\mathrm{j}}, \vec{\alpha}_{\mathcal{H}_{\mathrm{j}}}\right)$ of type $\mathrm{M}_{\mathcal{H}_{\mathrm{j}}}$ over $\overrightarrow{\mathrm{M}}_{\mathcal{H}, \Sigma}^{\text {tor }}$ (see [30, Definition 5.3.2.1]), whose pullback to $\mathrm{M}_{\mathcal{H}} \cong \mathrm{M}_{\mathcal{H}_{\mathrm{j}}}$ (see (2.1)) is isomorphic to the tautological object $\left(A_{\mathrm{j}}, \lambda_{\mathrm{j}}, i_{\mathrm{j}}, \alpha_{\mathcal{H}_{\mathrm{j}}}\right)$ over $\mathrm{M}_{\mathcal{H}_{\mathrm{j}}}$, and whose pullback to $\overrightarrow{\mathrm{M}}_{\mathcal{H}}$ is isomorphic to the degenerating family of type $\mathrm{M}_{\mathcal{H}_{\mathrm{j}}}$ over 
$\overrightarrow{\mathrm{M}}_{\mathcal{H}}$ which was denoted $\left(\vec{A}_{\mathrm{j}}, \vec{\lambda}_{\mathrm{j}}, \vec{i}_{\mathrm{j}}, \vec{\alpha}_{\mathcal{H}_{\mathrm{j}}}\right)$ in Proposition 6.1 (the notations for $\vec{\lambda}_{\mathrm{j}}$, $\vec{i}_{\mathrm{j}}$, and $\vec{\alpha}_{\mathcal{H}_{\mathrm{j}}}$ have been, unfortunately, overloaded and dependent on the context). For each $\left(\Phi_{\mathcal{H}}, \delta_{\mathcal{H}}, \sigma\right)$, the pullback of $\left(\vec{G}_{\mathrm{j}}, \vec{\lambda}_{\mathrm{j}}, \vec{i}_{\mathrm{j}}, \vec{\alpha}_{\mathcal{H}_{\mathrm{j}}}\right)$ to $\overrightarrow{\mathfrak{X}}_{\Phi_{\mathcal{H}}, \delta_{\mathcal{H}}, \sigma}$ via (10.3) (see Theorem 10.13) is canonically isomorphic to the Mumford family (8.29).

Proof. Using the incidence relation among the locally closed strata of $\overrightarrow{\mathrm{M}}_{\mathcal{H}, \Sigma}^{\text {tor }}$ in Theorem 9.13, we can write $\overrightarrow{\mathrm{M}}_{\mathcal{H}, \Sigma}^{\text {tor }}$ as a finite increasing union $U_{0} \subset U_{1} \subset \cdots$ of open subschemes such that $U_{0}=\overrightarrow{\mathrm{M}}_{\mathcal{H}}$ and such that, for each $i \geqslant 0$, the complement of $U_{i}$ in $U_{i+1}$ is some stratum $\vec{Z}_{\left[\left(\Phi_{\mathcal{H}}, \delta_{\mathcal{H}}, \sigma\right)\right]}$ closed in $U_{i+1}$. Then the theorem follows by repeatedly applying Lemma 11.1, with $\left(U, U^{\prime}\right)=\left(U_{i+1}, U_{i}\right)$ for (finitely many) increasing $i \geqslant 0$.

REMARK 11.3. The usual approximation and gluing arguments in [10, Ch. VI] and [30, Section 6.3] play no role in the proofs of Lemma 11.1 and Theorem 11.2. This is because we constructed the base scheme $\overrightarrow{\mathrm{M}}_{\mathcal{H}, \Sigma}^{\text {tor }}$ by taking normalizations in certain auxiliary models of proper smooth toroidal compactifications, and the approximation and gluing arguments are already used in the construction of such auxiliary models. On the contrary, since the approximation and gluing arguments require the extended Kodaira-Spencer morphisms to be defined, it is not even clear how they should work for the generally very singular local charts constructed in Section 8.

ThEOREM 11.4 (Cf. [30, Theorem 6.4.1.1(6)] and Theorem 7.14). With the setting as in Theorem 7.14, let $S$ be an irreducible noetherian normal scheme over $\overrightarrow{\mathrm{S}}_{0}=\operatorname{Spec}\left(\mathcal{O}_{F_{0},(p)}\right)$, with generic point $\eta$, which is equipped with a morphism

$$
\eta \rightarrow \mathrm{M}_{\mathcal{H}}
$$

Let $\left(A_{\eta}, \lambda_{\eta}, i_{\eta}, \alpha_{\mathcal{H}, \eta}\right)$ denote the pullback of the tautological object of $\mathrm{M}_{\mathcal{H}}$ to $\eta$ under (7.15). Suppose that, for each $\mathrm{j} \in \mathrm{J}$, we have a degenerating family $\left(G_{\mathrm{j}}^{\dagger}\right.$, $\left.\lambda_{\mathrm{j}}^{\dagger}, i_{\mathrm{j}}^{\dagger}, \alpha_{\mathcal{H}_{\mathrm{j}}}^{\dagger}\right)$ of type $\mathrm{M}_{\mathcal{H}_{\mathrm{j}}}$ over $S$, whose pullback $\left(G_{\mathrm{j}, \eta}, \lambda_{\mathrm{j}, \eta}, i_{\mathrm{j}, \eta}, \alpha_{\mathcal{H}_{\mathrm{j}}, \eta}\right)$ to $\eta$ defines a morphism

$$
\eta \rightarrow \mathrm{M}_{\mathcal{H}_{\mathrm{j}}}
$$

by the universal property of $\mathrm{M}_{\mathcal{H}_{\mathrm{j}}}$, which we assume to coincide with the composition of (11.5) with (2.1). Then (11.5) (necessarily uniquely) extends to a morphism $S \rightarrow \overrightarrow{\mathrm{M}}_{\mathcal{H}, \Sigma}^{\text {tor }}$ (over $\overrightarrow{\mathrm{S}}_{0}$ ) if and only if the same condition in the second last paragraph of Theorem 7.14 is satisfied at each geometric point $\bar{s}$ of $S$. 
Proof. For each $\mathrm{j} \in \mathrm{J}$, by the same construction as in the proof of Proposition 5.24, the degenerating family $\left(G_{\mathrm{j}}^{\dagger}, \lambda_{\mathrm{j}}^{\dagger}, i_{\mathrm{j}}^{\dagger}, \alpha_{\mathcal{H}_{\mathrm{j}}}^{\dagger}\right)$ of type $\mathrm{M}_{\mathcal{H}_{\mathrm{j}}}$ over $S$ induces a degenerating family $\left(G_{\mathrm{j}, \text { aux }}^{\dagger}, \lambda_{\mathrm{j} \text {,aux }}^{\dagger}, i_{\mathrm{j}, \text { aux }}^{\dagger}, \alpha_{\mathcal{H}_{\mathrm{j}, \text { aux }}}^{\dagger}\right)$ of type $\mathrm{M}_{\mathcal{H}_{\mathrm{j} \text {,aux }}}$ over $S$, which is determined up to unique isomorphism by its restriction to $\eta$, by the noetherian normality of $S$ and by [52, IX, 1.4], [10, Ch. I, Proposition 2.7], or [30, Proposition 3.3.1.5]. Hence this theorem follows from Theorems 7.14 and 11.2.

COROLlaRY 11.7. Up to canonical isomorphism, the scheme $\overrightarrow{\mathrm{M}}_{\mathcal{H}, \Sigma}^{\text {tor }}$ constructed in Section 7 depends only on the choices of the linear algebraic data in Section 2, but not on the auxiliary choices in Sections 4 and 5.

Proof. This is because the improved universal property of $\overrightarrow{\mathrm{M}}_{\mathcal{H}, \Sigma}^{\text {tor }}$ in Theorem 11.4 does not involve the auxiliary choices in Sections 4 and 5 at all.

COROLlaRY 11.8. In Lemma 9.8, if $\Sigma$ is already smooth (and satisfies [30, Condition 6.3.3.2]) as in [30, Definition 6.3.3.4], and if we take $\Sigma^{\prime}=\Sigma$ there, then the canonical morphism (9.9) is an isomorphism, identifying the subschemes $\mathrm{Z}_{\left[\left(\Phi_{\mathcal{H}}, \delta_{\mathcal{H}}, \sigma\right)\right]}$ on both sides, so that the stratification of $\mathrm{M}_{\mathcal{H}, \Sigma}^{\text {tor }}$ there coincides with the one in [30, Theorem 6.4.1.1(2)] (this finally justifies the notation of $\mathrm{M}_{\mathcal{H}, \Sigma}^{\text {tor }}$ and $\mathrm{Z}_{\left[\left(\Phi_{\mathcal{H}}, \delta \mathcal{H}, \sigma\right)\right]}$ there $)$.

Proof. This is because, by Lemma 3.21, the universal properties of $\mathrm{M}_{\mathcal{H}, \Sigma^{\prime}}^{\text {tor }}$ and $\mathrm{M}_{\mathcal{H}, \Sigma}^{\text {tor }}$ in [30, Theorem 6.4.1.1(6)] and Theorem 11.4, respectively, imply each other.

COROLlary 11.9 (Generalization of Corollary 10.18). If $\left[\left(\Phi_{\mathcal{H}}^{\prime}, \delta_{\mathcal{H}}^{\prime}, \sigma^{\prime}\right)\right]$ is a face of $\left[\left(\Phi_{\mathcal{H}}, \delta_{\mathcal{H}}, \sigma\right)\right]$ (see [30, Definition 6.3.2.14 and Theorem 6.4.1.1(2)]), in which case $\overrightarrow{\mathrm{Z}}_{\left[\left(\Phi_{\mathcal{H}}, \delta_{\mathcal{H}}, \sigma\right)\right]}$ is contained in the closure of $\overrightarrow{\mathrm{Z}}_{\left[\left(\Phi_{\mathcal{H}}^{\prime}, \delta_{\mathcal{H}}^{\prime}, \sigma^{\prime}\right)\right]}$ in $\overrightarrow{\mathrm{M}}_{\mathcal{H}, \Sigma}^{\text {tor }}$, then $\vec{Z}_{\left[\left(\Phi_{\mathcal{H}}, \delta_{\mathcal{H}}, \sigma\right)\right]} \otimes_{\mathbb{Z}} \mathbb{F}_{p}$ is also contained in the closure of $\vec{Z}_{\left[\left(\Phi_{\mathcal{H}}^{\prime}, \delta_{\mathcal{H}}^{\prime}, \sigma^{\prime}\right)\right]} \otimes_{\mathbb{Z}} \mathbb{F}_{p}$ in $\overrightarrow{\mathrm{M}}_{\mathcal{H}, \Sigma}^{\text {tor }} \otimes_{\mathbb{Z}} \mathbb{F}_{p}$.

Proof. Suppose $s$ is a point of $\vec{Z}_{\left[\left(\Phi_{\mathcal{H}}, \delta_{\mathcal{H}}, \sigma\right)\right]} \otimes_{\mathbb{Z}} \mathbb{F}_{p}$. By Corollary 10.15, $s$ can be identified with a point $t$ of $\vec{\Xi}_{\Phi_{\mathcal{H}}, \delta_{\mathcal{H}}, \sigma} \otimes_{\mathbb{Z}} \mathbb{F}_{p}$, where $\left(\Phi_{\mathcal{H}}, \delta_{\mathcal{H}}, \sigma\right)$ is some representative of $\left[\left(\Phi_{\mathcal{H}}, \delta_{\mathcal{H}}, \sigma\right)\right]$. Let $\left(\Phi_{\mathcal{H}}^{\prime}, \delta_{\mathcal{H}}^{\prime}, \sigma^{\prime}\right)$ be a representative of $\left[\left(\Phi_{\mathcal{H}}^{\prime}\right.\right.$, $\left.\left.\delta_{\mathcal{H}}^{\prime}, \sigma^{\prime}\right)\right]$. By assumption, there is a surjection from $\left(\Phi_{\mathcal{H}}^{\prime}, \delta_{\mathcal{H}}^{\prime}\right)$ to $\left(\Phi_{\mathcal{H}}, \delta_{\mathcal{H}}\right)$ such that the induced morphism $\mathbf{P}_{\Phi_{\mathcal{H}^{\prime}}} \rightarrow \mathbf{P}_{\Phi_{\mathcal{H}}}$ maps $\sigma^{\prime}$ to a face $\tau$ of $\sigma . \operatorname{Let} \operatorname{Spf}(R, I)$ be an affine open subscheme of $\overrightarrow{\mathfrak{X}}_{\Phi_{\mathcal{H}}, \delta_{\mathcal{H}}, \sigma}$ whose underlying topological space 
contains $t$. Let $t^{\prime}$ be any point in the preimage of $\vec{\Xi}_{\Phi_{\mathcal{H}}, \delta_{\mathcal{H}}, \tau} \otimes_{\mathbb{Z}} \mathbb{F}_{p}$ under the canonical morphism $\operatorname{Spec}(R) \rightarrow \vec{\Xi}_{\Phi_{\mathcal{H}}, \delta_{\mathcal{H}}}(\sigma)$ such that $t$ is contained in the closure of $t^{\prime}$. By considering the pullback of the Mumford family to the localization of $\operatorname{Spec}(R)$ at $t^{\prime}$, by the theory of two-step degenerations (see [10, Ch. III, Section 10] and [30, Section 4.5.6]), by the defining property of $\vec{Z}_{\left[\left(\Phi_{\mathcal{H}}^{\prime}, \delta_{\mathcal{H}}^{\prime}, \sigma^{\prime}\right)\right]}$ as in Definition 9.1, and by the universal property of $\overrightarrow{\mathrm{M}}_{\mathcal{H}, \Sigma}^{\text {tor }}$ as in Theorems 7.14 and 11.4 , the canonical morphism $\operatorname{Spec}(R) \rightarrow \overrightarrow{\mathrm{M}}_{\mathcal{H}, \Sigma}^{\text {tor }}$ maps $t^{\prime}$ to a point $s^{\prime}$ of $\vec{Z}_{\left[\left(\Phi_{\mathcal{H}}^{\prime}, \delta_{\mathcal{H}}^{\prime}, \sigma^{\prime}\right)\right]} \otimes_{\mathbb{Z}} \mathbb{F}_{p}$, so that $s$ is contained in the closure of $s^{\prime}$. Since $s$ is arbitrary, $\vec{Z}_{\left[\left(\Phi_{\mathcal{H}}, \delta_{\mathcal{H}}, \sigma\right)\right]} \otimes_{\mathbb{Z}} \mathbb{F}_{p}$ is contained in the closure of $\vec{Z}_{\left[\left(\Phi_{\mathcal{H}}^{\prime}, \delta_{\mathcal{H}}^{\prime}, \sigma^{\prime}\right)\right]} \otimes_{\mathbb{Z}} \mathbb{F}_{p}$, as desired.

\section{Stratifications of minimal compactifications}

The main goal of this section is to show that analogs of [30, Theorem 7.2.4.1 (4) and (5)] are true for $\vec{M}_{\mathcal{H}}^{\min }$ (see Theorems 12.1 and 12.16 below).

THEOREM 12.1 (Cf. [30, Theorem 7.2.4.1 (4) and (5)]). With the setting as above, the locally closed subschemes $\vec{Z}_{\left[\left(\Phi_{\mathcal{H}}, \delta_{\mathcal{H}}\right)\right]}$ of $\overrightarrow{\mathrm{M}}_{\mathcal{H}}^{\min }$ (see (6.8); cf. Lemma 6.9 and Remark 6.10), with $\left[\left(\Phi_{\mathcal{H}}, \delta_{\mathcal{H}}\right)\right]$ running through a complete set of cusp labels, form a stratification

$$
\overrightarrow{\mathrm{M}}_{\mathcal{H}}^{\min }=\coprod_{\left[\left(\Phi_{\mathcal{H}}, \delta_{\mathcal{H}}\right)\right]} \overrightarrow{\mathrm{Z}}_{\left[\left(\Phi_{\mathcal{H}}, \delta_{\mathcal{H}}\right)\right]}
$$

of $\overrightarrow{\mathrm{M}}_{\mathcal{H}}^{\min }$, such that the $\left[\left(\Phi_{\mathcal{H}}^{\prime}, \delta_{\mathcal{H}}^{\prime}\right)\right]$-stratum $\overrightarrow{\mathrm{Z}}_{\left[\left(\Phi_{\mathcal{H}}^{\prime}, \delta_{\mathcal{H}}^{\prime}\right)\right]}$ lies in the closure of the $\left[\left(\Phi_{\mathcal{H}}, \delta_{\mathcal{H}}\right)\right]$-stratum $\vec{Z}_{\left[\left(\Phi_{\mathcal{H}}, \delta_{\mathcal{H}}\right)\right]}$ if and only if there is a surjection from the cusp label $\left[\left(\Phi_{\mathcal{H}}^{\prime}, \delta_{\mathcal{H}}^{\prime}\right)\right]$ to the cusp label $\left[\left(\Phi_{\mathcal{H}}, \delta_{\mathcal{H}}\right)\right]$ as in [30, Definition 5.4.2.13] (the notation ' $\amalg$ ' only means a set-theoretic disjoint union. The algebro-geometric structure is still that of $\overrightarrow{\mathrm{M}}_{\mathcal{H}}^{\text {min }}$ ). The open dense subscheme $\overrightarrow{\mathrm{M}}_{\mathcal{H}}$ of $\overrightarrow{\mathrm{M}}_{\mathcal{H}}^{\text {min }}$ (see Proposition 6.4) coincides with the $[(0,0)]$-stratum $\vec{Z}_{[(0,0)]}$.

For each representative $\left(\mathrm{Z}_{\mathcal{H}}, \Phi_{\mathcal{H}}, \delta_{\mathcal{H}}\right)$ of cusp label and for each $\sigma \in \Sigma_{\Phi_{\mathcal{H}}}$ such that $\sigma \subset \mathbf{P}_{\Phi_{\mathcal{H}}}^{+}$, the restriction of $(7.12)$ to the $\left[\left(\Phi_{\mathcal{H}}, \delta_{\mathcal{H}}, \sigma\right)\right]$-stratum $\vec{Z}_{\left[\left(\Phi_{\mathcal{H}}, \delta_{\mathcal{H}}, \sigma\right)\right]}$ of $\overrightarrow{\mathrm{M}}_{\mathcal{H}, \Sigma}^{\text {tor }}$ as in Theorem 9.13 induces a canonical surjection

$$
\overrightarrow{\mathrm{Z}}_{\left[\left(\Phi_{\mathcal{H}}, \delta_{\mathcal{H}}, \sigma\right)\right]} \rightarrow \overrightarrow{\mathrm{Z}}_{\left[\left(\Phi_{\mathcal{H}}, \delta_{\mathcal{H}}\right)\right]} .
$$

Proof. The morphism (7.12) is proper and surjective because $\overrightarrow{\mathrm{M}}_{\mathcal{H}, \Sigma}^{\text {tor }}$ is proper over $\vec{S}_{0}$, and because the restriction of (7.12) induces the identity morphism on $\overrightarrow{\mathrm{M}}_{\mathcal{H}}$. By Lemma 9.8, for any smooth refinement $\Sigma^{\prime}$ of $\Sigma$, and for each $\left(\mathrm{Z}_{\mathcal{H}}, \Phi_{\mathcal{H}}, \delta_{\mathcal{H}}\right)$ 
and $\sigma$ as in the statement of the proposition, $Z_{\left[\left(\Phi_{\mathcal{H}}, \delta_{\mathcal{H}}, \sigma\right)\right]}=\vec{Z}_{\left[\left(\Phi_{\mathcal{H}}, \delta_{\mathcal{H}}, \sigma\right)\right]} \otimes_{\mathbb{Z}} \mathbb{Q}$ is the union of the images of the strata $Z_{\left[\left(\Phi_{\mathcal{H}}, \delta_{\mathcal{H}}, \tau\right)\right]}$ under (9.9), with $\tau$ running through the cones in $\Sigma_{\Phi_{\mathcal{H}}}^{\prime}$ that are contained in $\sigma$.

Let $\omega_{\mathrm{M}_{\mathcal{H}, \Sigma}^{\text {tor }}}$ denote the pullback of $\omega_{\mathrm{M}_{\mathcal{H}}^{\min }}$. By Propositions 6.4 and 7.11, $\omega_{\mathrm{M}_{\mathcal{H}, \Sigma}^{\text {tor }}}^{\otimes a_{\mathrm{J}}}$ is canonically isomorphic to the pullback of $\omega_{\overrightarrow{\mathrm{M}}_{\mathcal{H}, \Sigma}^{\text {tor }}}$, where $a_{\mathrm{J}}=\sum_{\mathrm{j} \in \mathrm{J}} a_{\mathrm{j}}$ is as in Proposition 6.1. Moreover, since (9.9) is defined by the universal property of $\mathrm{M}_{\mathcal{H}, \Sigma}^{\text {tor }}$ (by Proposition 5.24 and Theorem 7.14), the invertible sheaf $\omega_{\mathrm{M}_{\mathcal{H}, \Sigma^{\prime}}^{\text {tor }}}^{\otimes a_{\mathrm{J}}}$ over $\mathrm{M}_{\mathcal{H}, \Sigma^{\prime}}^{\text {tor }}$ is canonically isomorphic to the pullback of $\omega_{\mathrm{M}_{\mathcal{H}, \Sigma}^{\text {to }}}^{\otimes a_{\mathrm{J}}}$. For the sake of clarity, let us denote by $\oint_{\mathcal{H}, \Sigma}: \mathrm{M}_{\mathcal{H}, \Sigma}^{\text {tor }} \rightarrow \mathrm{M}_{\mathcal{H}}^{\text {min }}$ the morphism induced by (7.12), and denote by $\oint_{\mathcal{H}, \Sigma^{\prime}}: \mathrm{M}_{\mathcal{H}, \Sigma^{\prime}}^{\text {tor }} \rightarrow \mathrm{M}_{\mathcal{H}}^{\text {min }}$ the morphism (for $\mathrm{M}_{\mathcal{H}, \Sigma^{\prime}}^{\text {tor }}$ ) in [30, Theorem 7.2.4.1(3)]. Then it follows from Proposition 7.11 and from [30, Theorem 7.2.4.1(3)] that $\oint_{\mathcal{H}, \Sigma^{\prime}}$ coincides with the composition of (9.9) with $\oint_{\mathcal{H}, \Sigma}$. Since the restriction of $\oint_{\mathcal{H}, \Sigma^{\prime}}$ induces a surjective morphism from each $Z_{\left[\left(\Phi_{\mathcal{H}}, \delta_{\mathcal{H}}, \tau\right)\right]}$ as in the first paragraph to the stratum $Z_{\left[\left(\Phi_{\mathcal{H}}, \delta_{\mathcal{H}}\right)\right]}$ of $\mathrm{M}_{\mathcal{H}}^{\min }$ (see [30, Theorem 7.2.4.1(5)]), it follows that $\oint_{\mathcal{H}, \Sigma}$ induces a surjective morphism from $Z_{\left[\left(\Phi_{\mathcal{H}}, \delta_{\mathcal{H}}, \sigma\right)\right]}$ to $Z_{\left[\left(\Phi_{\mathcal{H}}, \delta_{\mathcal{H}}\right)\right]}$.

Since $Z_{\left[\left(\Phi_{\mathcal{H}}, \delta_{\mathcal{H}}, \sigma\right)\right]}=\vec{Z}_{\left[\left(\Phi_{\mathcal{H}}, \delta_{\mathcal{H}}, \sigma\right)\right]} \otimes_{\mathbb{Z}} \mathbb{Q}$ is dense in $\vec{Z}_{\left[\left(\Phi_{\mathcal{H}}, \delta_{\mathcal{H}}, \sigma\right)\right]}$ by Lemma 9.11, and since (7.12) is proper and surjective, it follows from the above (with $\vec{Z}_{\left[\left(\Phi_{\mathcal{H}}, \delta_{\mathcal{H}}, \sigma\right)\right]}$ running through all strata of $\left.\overrightarrow{\mathrm{M}}_{\mathcal{H}, \Sigma}^{\text {tor }}\right)$ that $Z_{\left[\left(\Phi_{\mathcal{H}}, \delta_{\mathcal{H}}\right)\right]}$ is isomorphic to $\vec{Z}_{\left[\left(\Phi_{\mathcal{H}}, \delta_{\mathcal{H}}\right)\right]} \otimes_{\mathbb{Z}} \mathbb{Q}$ and is dense in $\vec{Z}_{\left[\left(\Phi_{\mathcal{H}}, \delta_{\mathcal{H}}\right)\right]}$, that $\overrightarrow{\mathrm{M}}_{\mathcal{H}}^{\text {min }}$ is the union of $\vec{Z}_{\left[\left(\Phi_{\mathcal{H}}, \delta_{\mathcal{H}}\right)\right]}$ with $\left[\left(\Phi_{\mathcal{H}}, \delta_{\mathcal{H}}\right)\right]$ running through all cusp labels of $\mathrm{M}_{\mathcal{H}}$, and that this union defines a stratification of $\overrightarrow{\mathrm{M}}_{\mathcal{H}}^{\min }$ (then the incidence relations as in the statement of the proposition are forced by those of the stratification of $\mathrm{M}_{\mathcal{H}}^{\min }$ as in [30, Theorem 7.2.4.1(4)]).

By combining all of these, the last paragraph of the theorem also follows.

REMARK 12.3. Theorem 12.1 is rather incomplete compared with [30, Theorem 7.2.4.1 (4) and (5)]. It will be complemented by Theorem 12.16 below.

Nevertheless, there are already several useful consequences of Theorem 12.1.

COROLlaRy 12.4 (Cf. Corollary 11.9). If there is a surjection from $\left[\left(\Phi_{\mathcal{H}}, \delta_{\mathcal{H}}\right)\right]$ to $\left[\left(\Phi_{\mathcal{H}}^{\prime}, \delta_{\mathcal{H}}^{\prime}\right)\right]$ (see [30, Definition 5.4.2.13 and Theorem 7.2.4.1(4)]), in which case $\vec{Z}_{\left[\left(\Phi_{\mathcal{H}}, \delta_{\mathcal{H}}\right)\right]}$ is contained in the closure of $\overrightarrow{\mathrm{Z}}_{\left[\left(\Phi_{\mathcal{H}}^{\prime}, \delta_{\mathcal{H}}^{\prime}\right)\right]}$ in $\overrightarrow{\mathrm{M}}_{\mathcal{H}}^{\min }$, then $\overrightarrow{\mathrm{Z}}_{\left[\left(\Phi_{\mathcal{H}}, \delta_{\mathcal{H}}\right)\right]} \otimes_{\mathbb{Z}}$ $\mathbb{F}_{p}$ is also contained in the closure of $\vec{Z}_{\left[\left(\Phi_{\mathcal{H}}^{\prime}, \delta_{\mathcal{H}}^{\prime}\right)\right]} \otimes_{\mathbb{Z}} \mathbb{F}_{p}$ in $\overrightarrow{\mathrm{M}}_{\mathcal{H}}^{\min } \otimes_{\mathbb{Z}} \mathbb{F}_{p}$.

Proof. This follows from Corollary 11.9 and Theorem 12.1. 
Corollary 12.5. $\overrightarrow{\mathrm{M}}_{\mathcal{H}} \otimes_{\mathbb{Z}} \mathbb{F}_{p}$ is dense in $\overrightarrow{\mathrm{M}}_{\mathcal{H}}^{\min } \otimes_{\mathbb{Z}} \mathbb{F}_{p}$.

Proof. This is a special case of Corollary 12.4. One can also deduce this more directly from Corollary 10.18 and Theorem 12.1.

REMARK 12.6. Corollary 12.5 can also be proved by constructing elevators as in [27], which can be viewed as a minimalistic analog of the boundary charts constructed in Section 8. See the proof of [31, Proposition 2.2.1.7] for the special case where $\mathbf{J}=\left\{\mathrm{j}_{0}\right\}$ and $\left(g_{\mathrm{j}_{0}}, L_{\mathrm{j}_{0}},\langle\cdot, \cdot\rangle_{\mathrm{j}_{0}}\right)=(1, L,\langle\cdot, \cdot\rangle)$ are as in Example 2.3.

COROLlary 12.7. Up to canonical isomorphism, the scheme $\overrightarrow{\mathrm{M}}_{\mathcal{H}}^{\min }$ constructed in Proposition 6.4 depends only on the choices of the linear algebraic data in Section 2, but not on the auxiliary choices in Sections 4 and 5.

Proof. By Proposition 6.4 and Corollary $12.5, \overrightarrow{\mathrm{M}}_{\mathcal{H}}^{\min }$ is flat over $\mathbb{Z}_{(p)}$ and is noetherian normal, and the complement of $\overrightarrow{\mathrm{M}}_{\mathcal{H}} \cup \mathrm{M}_{\mathcal{H}}^{\min }$ in $\overrightarrow{\mathrm{M}}_{\mathcal{H}}^{\text {min }}$ is of codimension at least two. Hence the canonical restriction morphism

$$
\Gamma\left(\overrightarrow{\mathrm{M}}_{\mathcal{H}}^{\min }, \omega_{\overrightarrow{\mathrm{M}}_{\mathcal{H}}^{\min , \mathrm{J}}}^{\otimes k}\right) \rightarrow \Gamma\left(\overrightarrow{\mathrm{M}}_{\mathcal{H}} \cup \mathrm{M}_{\mathcal{H}}^{\min },\left.\omega_{\overrightarrow{\mathrm{M}}_{\mathcal{H}}^{\min }, \mathrm{J}}^{\otimes k}\right|_{\overrightarrow{\mathrm{M}}_{\mathcal{H}} \cup \mathrm{M}_{\mathcal{H}}^{\min }}\right)
$$

is an isomorphism for each $k \geqslant 0$. By Propositions 6.1 and 6.4, the right-hand side of (12.8) depends only on the choices of linear algebraic data in Section 2. Since $\overrightarrow{\mathrm{M}}_{\mathcal{H}}^{\min } \cong \operatorname{Proj}\left(\bigoplus_{k \geqslant 0} \Gamma\left(\overrightarrow{\mathrm{M}}_{\mathcal{H}}^{\min }, \omega_{\overrightarrow{\mathrm{M}}_{\mathcal{H}}^{\min }, \mathrm{J}}^{\otimes}\right)\right)$ by Proposition 6.4, the corollary follows.

By Proposition 7.11, $\omega_{\overrightarrow{\mathrm{M}}_{\mathcal{H}, \Sigma}^{\text {tor }}, \mathrm{J}}$ is canonically isomorphic to the pullback of $\bigotimes_{\mathrm{j} \in \mathrm{J}} \omega_{\mathrm{M}_{\mathcal{H}_{\mathrm{j}, \text { aux }}, \Sigma_{\mathrm{j}, \text { aux }}}^{\otimes a_{\mathrm{j}, 0}}}^{\text {under }}$ (7.8). By [52, IX, 1.4], [10, Ch. I, Proposition 2.7], or [30, Proposition 3.3.1.5], for each $\mathrm{j} \in \mathrm{J}$, the degenerating family $\left(\vec{G}_{\mathrm{j}}, \vec{\lambda}_{\mathrm{j}}, \vec{i}_{\mathrm{j}}, \vec{\alpha}_{\mathcal{H}_{\mathrm{j}}}\right)$ in Theorem 11.2 induces (up to canonical isomorphism) the pullback of the tautological tuple $\left(G_{\mathrm{j}, \text { aux }}, \lambda_{\mathrm{j} \text {,aux }}, i_{\mathrm{j}, \text { aux }}, \alpha_{\mathcal{H}_{\mathrm{j}, \text { aux }}}\right)$ over $\mathrm{M}_{\mathcal{H}_{\mathrm{j}, \text { aux }}, \Sigma_{\mathrm{j}, \text { aux }}}^{\text {to }}$ to $\overrightarrow{\mathrm{M}}_{\mathcal{H}, \Sigma}^{\text {tor }}$, because it is so over $\mathrm{M}_{\mathcal{H}}$. Hence, by the same argument as in the proof of Lemma 5.30, based on [39, IX, 2.4, and its proof], we have

$$
\omega_{\overrightarrow{\mathrm{M}}_{\mathcal{H}, \Sigma}^{\text {tor }}, \mathrm{J}} \cong \bigotimes_{\mathrm{j} \in \mathrm{J}} \omega_{\overrightarrow{\mathrm{j}}_{\mathrm{j}} / \mathrm{M}_{\mathcal{H}, \Sigma}^{\text {tor }}}^{\otimes a_{\mathrm{j}}}
$$

where

$$
\omega_{\vec{G}_{\mathrm{j}} / \overline{\mathrm{M}}_{\mathcal{H}, \Sigma}^{\text {tor }}}:=\wedge^{\text {top }} \underline{\mathrm{Lie}}_{\vec{G}_{\mathrm{j}} / \overrightarrow{\mathrm{M}}_{\mathcal{H}, \Sigma}^{\mathrm{tor}}}^{\vee}
$$


for each $\mathrm{j} \in \mathrm{J}$. Thus, if we set

$$
\vec{G}_{\mathrm{J}}:=\prod_{\mathrm{j} \in \mathrm{J}} \vec{G}_{\mathrm{j}}^{\times a_{\mathrm{j}}}
$$

where the products are fiber products over $\overrightarrow{\mathrm{M}}_{\mathcal{H}, \Sigma}^{\text {tor }}$, then we have

$$
\omega_{\overrightarrow{\mathrm{M}}_{\mathcal{H}, \Sigma}^{\text {tor }}, \mathrm{J}} \cong \wedge^{\text {top }} \underline{\mathrm{Lie}}_{\vec{G}_{\mathrm{J}} / \overrightarrow{\mathrm{M}}_{\mathcal{H}, \Sigma}^{\mathrm{tor}}}^{\vee}
$$

LEMMA 12.9. The fibers of (7.12) are all geometrically connected. The isomorphism class of the abelian part of $\vec{G}_{\mathrm{J}}$ is constant on each geometric fiber of (7.12).

Proof. The first assertion is because $\omega_{\overrightarrow{\mathrm{M}}_{\mathcal{H}, \Sigma}^{\text {tor }}, \mathrm{J}}$ is the pullback of the ample invertible sheaf $\omega_{\overrightarrow{\mathrm{M}}_{\mathcal{H}}^{\text {min }}, \mathrm{J}}$ (see Propositions 6.4 and 7.11), so that (7.12) is its own Stein factorization (see [14, III-1, 4.3.3 and 4.3.4]), by the normality of $\overrightarrow{\mathrm{M}}_{\mathcal{H}}^{\min }$ and by Zariski's main theorem (see [14, III-1, 4.4.3, 4.4.11]). The second assertion then follows from [30, Proposition 7.2.1.2], by the same arguments as in the beginning of [30, Section 7.2.3].

In the remainder of this section, our goal is to prove Theorem 12.16, which complements Theorem 12.1 and gives a more precise description of the strata.

For each $\left(\Phi_{\mathcal{H}}, \delta_{\mathcal{H}}, \sigma\right)$ (as in [30, Definition 6.2.6.1]) such that $\sigma \subset \mathbf{P}_{\Phi_{\mathcal{H}}}^{+}$and $\sigma \in \Sigma_{\Phi_{\mathcal{H}}} \in \Sigma$, let ${ }^{\circ} \omega_{\mathrm{J}}$ denote the pullback of $\omega_{\overrightarrow{\mathrm{M}}_{\mathcal{H}, \Sigma}^{\text {tor }}, \mathrm{J}}$ under (8.33). Let

$$
\vec{B}_{\mathrm{J}}:=\prod_{\mathrm{j} \in \mathrm{J}} \vec{B}_{\mathrm{j}}^{\times a_{\mathrm{j}}}
$$

where the products are fiber products over $\overrightarrow{\mathrm{M}}_{\mathcal{H}}^{\mathrm{Z}_{\mathcal{H}}}$. Consider the invertible sheaf

$$
{ }^{\ominus} \omega_{\mathrm{J}}^{\natural}:=\left(\bigotimes_{\mathrm{j} \in \mathrm{J}}\left(\wedge_{\mathbb{Z}}^{\text {top }} X_{\mathrm{j}}\right)^{\otimes a_{\mathrm{j}}}\right) \underset{\mathbb{Z}}{\otimes}\left(\wedge^{\text {top }} \underline{\mathrm{Lie}}_{\vec{B}_{\mathrm{J}} / \overrightarrow{\mathrm{M}}_{\mathcal{H}, \Sigma}^{\text {tor }}}\right)
$$

over $\overrightarrow{\mathrm{M}}_{\mathcal{H}}^{\mathrm{ZH}}$. By the same argument as in the proof of [30, Lemma 7.1.2.1], ${ }^{\circ} \omega_{\mathrm{J}}$ is canonically isomorphic to the pullback of ${ }^{\ominus} \omega_{\mathrm{J}}^{\natural}$. By considering the FourierJacobi expansions and by the same arguments as in [30, Section 7.1.2], we obtain the following. 
Proposition 12.10 (Cf. [30, Proposition 7.1.2.13]). For each $k \geqslant 0$, and for each $f \in \Gamma\left(\overrightarrow{\mathrm{M}}_{\mathcal{H}, \Sigma}^{\text {tor }}, \omega_{\overrightarrow{\mathrm{M}}_{\mathcal{H}, \Sigma}^{\text {tor }}, \mathrm{J}}^{\otimes k}\right)$, the pullback of $f$ to the $\left[\left(\Phi_{\mathcal{H}}, \delta_{\mathcal{H}}, \sigma\right)\right]$-stratum $\overrightarrow{\mathrm{Z}}_{\left[\left(\Phi_{\mathcal{H}}, \delta_{\mathcal{H}}, \sigma\right)\right]}$ of $\overrightarrow{\mathrm{M}}_{\mathcal{H}, \Sigma}^{\text {tor }}$ is constant along the fibers of the structural morphism

$$
\overrightarrow{\mathrm{Z}}_{\left[\left(\Phi_{\mathcal{H}}, \delta_{\mathcal{H}}, \sigma\right)\right]} \cong \vec{\Xi}_{\Phi_{\mathcal{H}}, \delta_{\mathcal{H}}, \sigma} \rightarrow \overrightarrow{\mathrm{M}}_{\mathcal{H}}^{\mathrm{Z}_{\mathcal{H}}}
$$

(see Corollary 10.15 for the first isomorphism).

COROllary 12.12 (Cf. [30, Corollary 7.2.3.12]). The morphism (12.2) factors through (12.11) and induces a canonical surjection

$$
\overrightarrow{\mathrm{M}}_{\mathcal{H}}^{\mathrm{Z}_{\mathcal{H}}} \rightarrow \overrightarrow{\mathrm{Z}}_{\left[\left(\Phi_{\mathcal{H}}, \delta_{\mathcal{H}}\right)\right]}
$$

This surjection is finite and induces a canonical isomorphism from $\overrightarrow{\mathrm{M}}_{\mathcal{H}}^{Z \mathcal{H}}$ to the normalization of $\vec{Z}_{\left[\left(\Phi_{\mathcal{H}}, \delta_{\mathcal{H}}\right)\right]}$.

Proof. The first assertion follows from Proposition 12.10. Since the isomorphism class of the abelian part of $\vec{G}_{\mathrm{J}}$ is constant on each geometric fiber of (7.12) (see Lemma 12.9), it follows from the finiteness of (7.6) that the induced morphism (12.13) is quasifinite. Since (12.13) induces the canonical isomorphism $\mathrm{M}_{\mathcal{H}}^{Z_{\mathcal{H}}} \stackrel{\sim}{\rightarrow}$ $\mathrm{Z}_{\left[\left(\Phi_{\mathcal{H}}, \delta_{\mathcal{H}}\right)\right]}$ in characteristic zero (see [30, Corollary 7.2.3.18]), the second assertion follows from Zariski's main theorem (see [14, III-1, 4.4.3, 4.4.11]).

Proposition 12.14 (Cf. [30, Proposition 7.2.3.16]). Let $\bar{x}$ be a geometric point of $\overrightarrow{\mathrm{M}}_{\mathcal{H}}^{\min }$ over the $\left[\left(\Phi_{\mathcal{H}}, \delta_{\mathcal{H}}\right)\right]$-stratum $\mathrm{Z}_{\left[\left(\Phi_{\mathcal{H}}, \delta_{\mathcal{H}}\right)\right]}$. Let $\left(\overrightarrow{\mathrm{M}}_{\mathcal{H}}^{\min }\right)_{\bar{x}}^{\wedge}$ denote the completion of the strict localization of $\overrightarrow{\mathrm{M}}_{\mathcal{H}}^{\min }$ at $\bar{x}$, let

$$
\left(\vec{Z}_{\left[\left(\Phi_{\mathcal{H}}, \delta_{\mathcal{H}}\right)\right]}\right)_{\bar{x}}^{\wedge}:=\overrightarrow{\mathrm{Z}}_{\left[\left(\Phi_{\mathcal{H}}, \delta_{\mathcal{H}}\right)\right]} \underset{\overrightarrow{\mathrm{M}}_{\mathcal{H}}^{\min }}{\times}\left(\overrightarrow{\mathrm{M}}_{\mathcal{H}}^{\min }\right)_{\overline{\bar{x}}}^{\wedge},
$$

and let

$$
\left(\overrightarrow{\mathrm{M}}_{\mathcal{H}}^{Z_{\mathcal{H}}}\right)_{\bar{x}}^{\wedge}:=\overrightarrow{\mathrm{M}}_{\mathcal{H}}^{Z_{\mathcal{H}}} \underset{\overrightarrow{\mathrm{Z}}_{\left[\left(\Phi_{\mathcal{H}}, \delta_{\mathcal{H}}\right)\right]}}{\times}\left(\overrightarrow{\mathrm{Z}}_{\left[\left(\Phi_{\mathcal{H}}, \delta_{\mathcal{H}}\right)\right]}\right)_{\overline{\bar{x}}}^{\wedge} .
$$

For each $\ell \in \mathbf{S}_{\Phi_{\mathcal{H}}}$, let $\left(\overrightarrow{\mathrm{FJ}}_{\Phi_{\mathcal{H}}, \delta_{\mathcal{H}}}^{(\ell)}\right)_{\bar{x}}^{\wedge}$ denote the pullback of

$$
\overrightarrow{\mathrm{F}}_{\Phi_{\mathcal{H}}, \delta_{\mathcal{H}}}^{(\ell)}:=\left(\vec{C}_{\Phi_{\mathcal{H}}, \delta_{\mathcal{H}}} \rightarrow \overrightarrow{\mathrm{M}}_{\mathcal{H}}^{\mathrm{Z} \mathcal{H}}\right)_{*}\left(\vec{\Psi}_{\Phi_{\mathcal{H}}, \delta_{\mathcal{H}}}(\ell)\right)
$$

under the canonical morphism $\left(\overrightarrow{\mathrm{M}}_{\mathcal{H}}^{\mathrm{ZH}}\right)_{\bar{x}}^{\wedge} \rightarrow \overrightarrow{\mathrm{M}}_{\mathcal{H}}^{\mathrm{Z}_{\mathcal{H}}}$. Then we have a canonical isomorphism

$$
\mathscr{O}_{\left(\overrightarrow{\mathrm{M}}_{\mathcal{H}}^{\min }\right)_{\bar{x}}^{\wedge}} \cong\left(\prod_{\ell \in \mathbf{P}_{\Phi_{\mathcal{H}}}^{\vee}}\left(\underline{\overrightarrow{\mathrm{F}}}_{\Phi_{\mathcal{H}}, \delta_{\mathcal{H}}}^{(\ell)}\right)_{\bar{x}}^{\wedge}\right)^{\Gamma_{\Phi_{\mathcal{H}}}}
$$


where

$$
\mathbf{P}_{\Phi_{\mathcal{H}}}^{\vee}:=\left\{\ell \in \mathbf{S}_{\Phi_{\mathcal{H}}}:\langle\ell, y\rangle \geqslant 0 \forall y \in \mathbf{P}_{\Phi_{\mathcal{H}}}\right\}
$$

as usual, which is adic if we interpret the product on the right-hand side as the completion of the elements that are finite sums with respect to the ideal generated by the elements without constant terms (that is, with trivial projection to $\left.\left(\overrightarrow{\mathrm{FJ}}_{\Phi_{\mathcal{H}}, \delta_{\mathcal{H}}}^{(0)}\right)_{\bar{x}}^{\wedge}\right)$. Then $(12.15)$ induces a homomorphism

$$
\left(\left(\underline{\mathrm{FJ}}_{\Phi_{\mathcal{H}}, \delta \mathcal{H}}^{(0)}\right)_{\bar{x}}^{\wedge}\right)^{\Gamma_{\Phi_{\mathcal{H}}}} \rightarrow \mathscr{O}_{\left(\overrightarrow{\mathrm{M}}_{\mathcal{H}}^{\min }\right)_{\bar{x}}^{\wedge}}
$$

whose source is canonically isomorphic to $\mathscr{O}_{\left(\overrightarrow{\mathrm{M}}_{\mathcal{H}}^{z}\right)_{\hat{x}}}$ (by Corollary 12.12 and Zariski's main theorem; see [14, III-1, 4.4.3, 4.4.11]). This defines a structural morphism

$$
\left(\overrightarrow{\mathrm{M}}_{\mathcal{H}}^{\min }\right)_{\bar{x}}^{\wedge} \rightarrow\left(\overrightarrow{\mathrm{M}}_{\mathcal{H}}^{\mathrm{Z}_{\mathcal{H}}}\right)_{\bar{x}}^{\wedge}
$$

whose precomposition with the canonical morphism $\left(\overrightarrow{\mathrm{Z}}_{\left[\left(\Phi_{\mathcal{H}}, \delta_{\mathcal{H}}\right)\right]}\right)_{\bar{x}}^{\wedge} \rightarrow\left(\overrightarrow{\mathrm{M}}_{\mathcal{H}}^{\min }\right)_{\bar{x}}^{\wedge}$ defines a canonical morphism

$$
\left(\overrightarrow{\mathrm{Z}}_{\left[\left(\Phi_{\mathcal{H}}, \delta \mathcal{H}\right)\right]}\right)_{\bar{x}}^{\wedge} \rightarrow\left(\overrightarrow{\mathrm{M}}_{\mathcal{H}}^{\mathrm{Z}_{\mathcal{H}}}\right)_{\bar{x}}^{\wedge}
$$

which is an isomorphism because its precomposition with the completion

$$
\left(\overrightarrow{\mathrm{M}}_{\mathcal{H}}^{\mathrm{Z}_{\mathcal{H}}}\right)_{\bar{x}}^{\wedge} \rightarrow\left(\overrightarrow{\mathrm{Z}}_{\left[\left(\Phi_{\mathcal{H}}, \delta_{\mathcal{H}}\right)\right]}\right)_{\bar{x}}^{\wedge}
$$

of (12.13) is the identity morphism on $\left(\overrightarrow{\mathrm{M}}_{\mathcal{H}}^{z_{\mathcal{H}}}\right)_{\bar{x}}^{\wedge}$. So, this last completion of (12.13) is also an isomorphism.

Proof. The same argument as in the proof of [30, Proposition 7.2.3.16] works here (we do not need to know a priori that (12.13) induces a bijection on geometric points).

THEOREM 12.16 (Cf. [30, Theorem 7.2.4.1 (4) and (5)]; continuation of Theorem 12.1). In the first paragraph of Theorem 12.1 , each $\left[\left(\Phi_{\mathcal{H}}, \delta_{\mathcal{H}}\right)\right]$ stratum $\vec{Z}_{\left[\left(\Phi_{\mathcal{H}}, \delta_{\mathcal{H}}\right)\right]}$ is canonically isomorphic to $\overrightarrow{\mathrm{M}}_{\mathcal{H}}^{\mathrm{Z}_{\mathcal{H}}}$. In the second paragraph of Theorem 12.1, the canonical surjection (12.2) can be identified with the composition of the canonical isomorphism $\vec{Z}_{\left[\left(\Phi_{\mathcal{H}}, \delta_{\mathcal{H}}, \sigma\right)\right]} \stackrel{\sim}{\rightarrow} \vec{\Xi}_{\Phi_{\mathcal{H}}, \delta_{\mathcal{H}}, \sigma}$ in Corollary 10.15, the structural morphism $\vec{\Xi}_{\Phi_{\mathcal{H}}, \delta_{\mathcal{H}}, \sigma} \rightarrow \overrightarrow{\mathrm{M}}_{\mathcal{H}}^{\mathrm{Z}_{\mathcal{H}}}$, and the isomorphism $\overrightarrow{\mathrm{M}}_{\mathcal{H}}^{\mathrm{Z}_{\mathcal{H}}} \stackrel{\sim}{\rightarrow} \overrightarrow{\mathrm{Z}}_{\left[\left(\Phi_{\mathcal{H}}, \delta_{\mathcal{H}}\right)\right]}$ mentioned above. In particular, it is proper if $\sigma$ is topdimensional in $\mathbf{P}_{\Phi_{\mathcal{H}}}^{+} \subset\left(\mathbf{S}_{\Phi_{\mathcal{H}}}\right)_{\mathbb{R}}^{\vee}$. 
Proof. Since (12.2) is the composition of (12.11) with (12.13), it suffices to show that (12.13) is an isomorphism. Since this assertion can be verified over formal completions of strict local rings, it follows from Proposition 12.14.

\section{Functorial properties and Hecke twists}

In this section, for the sake of clarity, we shall abusively denote all objects constructed using $\left\{\left(g_{\mathrm{j}}, L_{\mathrm{j}},\langle\cdot, \cdot\rangle_{\mathrm{j}}\right)\right\}_{\mathrm{j} \in \mathrm{J}}$ by an additional subscript J, such as $\overrightarrow{\mathrm{M}}_{\mathcal{H}, \mathrm{J}}$.

Proposition 13.1. Suppose that $\mathcal{H} \subset \mathcal{H}^{\prime}$ and $\mathbf{J}^{\prime} \subset \mathrm{J}$, and that the subcollection $\left\{\left(g_{\mathrm{j}}, L_{\mathrm{j}},\langle\cdot, \cdot\rangle_{\mathrm{j}}\right)\right\}_{\mathrm{j} \in \mathrm{J}^{\prime}}$ of $\left\{\left(g_{\mathrm{j}}, L_{\mathrm{j}},\langle\cdot, \cdot\rangle_{\mathrm{j}}\right)\right\}_{\mathrm{j} \in \mathrm{J}}$ satisfies the analogous conditions defined by $\mathcal{H}^{\prime}$ as in Section 2. Then there is a canonical morphism

$$
\overrightarrow{\mathrm{M}}_{\mathcal{H}, \mathrm{J}} \rightarrow \overrightarrow{\mathrm{M}}_{\mathcal{H}^{\prime}, \mathrm{J}^{\prime}}
$$

extending the canonical morphism

$$
\mathrm{M}_{\mathcal{H}} \rightarrow \mathrm{M}_{\mathcal{H}^{\prime}}
$$

Proof. This follows from the proof of Proposition 6.1, because $\overrightarrow{\mathrm{M}}_{\mathcal{H}, \mathrm{J}}$ (respectively $\left.\overrightarrow{\mathrm{M}}_{\mathcal{H}^{\prime}, J^{\prime}}\right)$ is the normalization of $\prod_{\mathrm{j} \in \mathrm{J}} \mathcal{A}_{g, d_{\mathrm{j}}} \otimes_{\mathbb{Z}} \mathbb{Z}_{(p)}$ (respectively $\prod_{\mathrm{j} \in \mathrm{J}^{\prime}} \mathcal{A}_{g, d_{\mathrm{j}}} \otimes_{\mathbb{Z}}$ $\mathbb{Z}_{(p)}$ ) under the canonical morphism $\mathrm{M}_{\mathcal{H}} \rightarrow \prod_{\mathrm{j} \in \mathrm{J}} \mathcal{A}_{g, d_{\mathrm{j}}} \otimes_{\mathbb{Z}} \mathbb{Z}_{(p)}$ (respectively $\left.\mathrm{M}_{\mathcal{H}^{\prime}} \rightarrow \prod_{\mathrm{j} \in \mathrm{J}^{\prime}} \mathcal{A}_{g, d_{\mathrm{j}}} \otimes_{\mathbb{Z}} \mathbb{Z}_{(p)}\right)$, and because $\mathrm{M}_{\mathcal{H}} \rightarrow \prod_{\mathrm{j} \in \mathrm{J}} \mathcal{A}_{g, d_{\mathrm{j}}} \otimes_{\mathbb{Z}} \mathbb{Z}_{(p)}$ and $\mathrm{M}_{\mathcal{H}^{\prime}} \rightarrow$ $\prod_{\mathrm{j} \in \mathrm{J}^{\prime}} \mathcal{A}_{g, d_{\mathrm{j}}} \otimes_{\mathbb{Z}} \mathbb{Z}_{(p)}$ are compatible with the canonical morphisms (13.3) and $\prod_{\mathrm{j} \in \mathrm{J}} \mathcal{A}_{g, d_{\mathrm{j}}} \otimes_{\mathbb{Z}} \mathbb{Z}_{(p)} \rightarrow \prod_{\mathrm{j} \in \mathrm{J}^{\prime}} \mathcal{A}_{g, d_{\mathrm{j}}} \otimes_{\mathbb{Z}} \mathbb{Z}_{(p)}$.

PROPOSITION 13.4. With the setting as in Proposition 13.1, there is a canonical morphism

$$
\overrightarrow{\mathrm{M}}_{\mathcal{H}, \mathrm{J}}^{\min } \rightarrow \overrightarrow{\mathrm{M}}_{\mathcal{H}^{\prime}, \mathrm{J}^{\prime}}^{\min }
$$

extending the canonical morphisms (13.2) and

$$
\mathrm{M}_{\mathcal{H}}^{\min } \rightarrow \mathrm{M}_{\mathcal{H}^{\prime}}^{\min }
$$

Proof. This is because, by Corollary 12.7, we may assume that $\mathcal{H}_{\mathrm{j} \text {, aux }} \subset \mathcal{H}_{\mathrm{j} \text {, aux }}^{\prime}$ and that the morphisms $\mathrm{M}_{\mathcal{H}}^{\min } \rightarrow \prod_{\mathrm{j} \in \mathrm{J}} \mathrm{M}_{\mathcal{H}_{\mathrm{j}, \text { aux }}}^{\min }$ and $\mathrm{M}_{\mathcal{H}^{\prime}}^{\min } \rightarrow \prod_{\mathrm{j} \in \mathrm{J}^{\prime}} \mathrm{M}_{\mathcal{H}_{\mathrm{j}, \text { aux }}^{\prime}}^{\min }$ used in the constructions of $\overrightarrow{\mathrm{M}}_{\mathcal{H}, \mathrm{J}}^{\min }$ and $\overrightarrow{\mathrm{M}}_{\mathcal{H}^{\prime}, \mathrm{J}^{\prime}}^{\min }$ (see Proposition 6.4) are compatible with the canonical morphisms (13.6) and $\prod_{\mathrm{j} \in \mathrm{J}} \mathrm{M}_{\mathcal{H}_{\mathrm{j}, \text { aux }}^{\min }}^{\operatorname{mo}} \rightarrow \prod_{\mathrm{j} \in \mathrm{J}^{\prime}} \mathrm{M}_{\mathcal{H}_{\mathrm{j}, \text { aux }}^{\prime}}^{\min }$. 
Proposition 13.7. With the setting as in Proposition 13.1, suppose moreover that $\Sigma$ and $\Sigma^{\prime}$ are compatible choices of admissible rational polyhedral cone decomposition data for $\mathrm{M}_{\mathcal{H}}$ and $\mathrm{M}_{\mathcal{H}}$, respectively, which are induced by certain auxiliary collections $\left\{\Sigma_{\mathrm{j}, \text { aux }}\right\}_{\mathrm{j} \in \mathrm{J}}$ and $\left\{\Sigma_{\mathrm{j}, \text { aux }}^{\prime}\right\}_{\mathrm{j} \in \mathrm{J}^{\prime}}$, as in Section 7 , such that $\Sigma$ is a 1-refinement of $\Sigma^{\prime}$ as in [30, Definition 6.4.3.3] (the definition there naturally generalizes to the case of nonsmooth cone decompositions). Then there is a canonical morphism

$$
\overrightarrow{\mathrm{M}}_{\mathcal{H}, \Sigma, \mathrm{J}}^{\text {tor }} \rightarrow \overrightarrow{\mathrm{M}}_{\mathcal{H}^{\prime}, \Sigma^{\prime}, \mathrm{J}^{\prime}}^{\text {tor }}
$$

extending the canonical morphisms (13.2) and is compatible with (13.5) under the canonical morphisms $\vec{\oint}_{\mathcal{H}, \mathrm{J}}: \overrightarrow{\mathrm{M}}_{\mathcal{H}, \Sigma, \mathrm{J}}^{\text {tor }} \rightarrow \overrightarrow{\mathrm{M}}_{\mathcal{H}, \mathrm{J}}^{\min }$ and $\vec{\oint}_{\mathcal{H}^{\prime}, \mathrm{J}^{\prime}}: \overrightarrow{\mathrm{M}}_{\mathcal{H}^{\prime}, \Sigma^{\prime}, \mathrm{J}^{\prime}}^{\text {tor }} \rightarrow \overrightarrow{\mathrm{M}}_{\mathcal{H}^{\prime}, \mathrm{J}^{\prime}}^{\text {min }}$.

Proof. The morphism (13.8) exists because, by Theorem 11.2, $\overrightarrow{\mathrm{M}}_{\mathcal{H}, \Sigma, \mathrm{J}}^{\text {tor }}$ carries the collection of degenerating families $\left\{\left(\vec{G}_{\mathrm{j}}, \vec{\lambda}_{\mathrm{j}}, \vec{i}_{\mathrm{j}}, \vec{\alpha}_{\mathcal{H}_{\mathrm{j}}}\right)\right\}_{\mathrm{j} \in \mathrm{J}}$, which induces a collection $\left\{\left(\vec{G}_{\mathrm{j}}, \vec{\lambda}_{\mathrm{j}}, \vec{i}_{\mathrm{j}}, \vec{\alpha}_{\mathcal{H}_{\mathrm{j}}^{\prime}}\right)\right\}_{\mathrm{j} \in \mathrm{J}^{\prime}}$ satisfying the universal property of $\overrightarrow{\mathrm{M}}_{\mathcal{H}^{\prime}, \Sigma^{\prime}, J^{\prime}}$ as in Theorems 7.14 and 11.4. To show that (13.8) is compatible with (13.5), it suffices to note that, by Corollary 11.7, we may assume that $\mathcal{H}_{\mathrm{j} \text {,aux }} \subset \mathcal{H}_{\mathrm{j} \text {,aux }}^{\prime}$ and that $\Sigma_{\mathrm{j} \text {,aux }}$ is a 1-refinement of $\Sigma_{\mathrm{j} \text {,aux }}^{\prime}$, for each $\mathrm{j} \in \mathrm{J}^{\prime}$, so that (13.8) and (13.5), together with the morphisms $\vec{\oint}_{\mathcal{H}, \mathrm{J}}$ and $\vec{\oint}_{\mathcal{H}^{\prime}, \mathrm{J}^{\prime}}$, are compatibly induced by the canonical morphisms $\prod_{\mathrm{j} \in \mathrm{J}} \mathrm{M}_{\mathcal{H}_{\mathrm{j}, \text { aux }}, \Sigma_{\mathrm{j}, \text { aux }}}^{\text {tor }} \rightarrow \prod_{\mathrm{j} \in \mathrm{J}^{\prime}} \mathrm{M}_{\mathcal{H}_{\mathrm{j}, \text { aux }}^{\prime} \Sigma_{\mathrm{j}, \text { aux }}^{\prime}}^{\text {tor }}$ and $\prod_{\mathrm{j} \in \mathrm{J}} \mathrm{M}_{\mathcal{H}_{\mathrm{j}, \text { aux }}}^{\min } \rightarrow \prod_{\mathrm{j} \in \mathrm{J}^{\prime}} \mathrm{M}_{\mathcal{H}_{\mathrm{j}, \text { aux }}^{\prime}}^{\min }$.

Proposition 13.9. Under (13.8), the $\left[\left(\Phi_{\mathcal{H}}, \delta_{\mathcal{H}}, \sigma\right)\right]$-stratum $\vec{Z}_{\left[\left(\Phi_{\mathcal{H}}, \delta_{\mathcal{H}}, \sigma\right)\right], \mathrm{J}}$ of $\overrightarrow{\mathrm{M}}_{\mathcal{H}, \Sigma, \mathrm{J}}^{\text {tor }}$ is mapped to the $\left[\left(\Phi_{\mathcal{H}^{\prime}}^{\prime}, \delta_{\mathcal{H}^{\prime}}, \sigma^{\prime}\right)\right]$-stratum $\overrightarrow{\mathrm{Z}}_{\left[\left(\Phi_{\mathcal{H}^{\prime}}^{\prime}, \delta_{\mathcal{H}^{\prime}}, \sigma^{\prime}\right)\right], J^{\prime}}$ of $\overrightarrow{\mathrm{M}}_{\mathcal{H}^{\prime}, \Sigma^{\prime}, J^{\prime}}^{\text {tor }}$ if and only if there are representatives $\left(\Phi_{\mathcal{H}}, \delta_{\mathcal{H}}, \sigma\right)$ and $\left(\Phi_{\mathcal{H}^{\prime}}^{\prime}, \delta_{\mathcal{H}^{\prime}}^{\prime}, \sigma^{\prime}\right)$ of the equivalence classes $\left[\left(\Phi_{\mathcal{H}}, \delta_{\mathcal{H}}, \sigma\right)\right]$ and $\left[\left(\Phi_{\mathcal{H}^{\prime}}^{\prime}, \delta_{\mathcal{H}^{\prime}}^{\prime}, \sigma^{\prime}\right)\right]$, respectively, such that $\left(\Phi_{\mathcal{H}}, \delta_{\mathcal{H}}, \sigma\right)$ is a 1-refinement of $\left(\Phi_{\mathcal{H}^{\prime}}^{\prime}, \delta_{\mathcal{H}^{\prime}}^{\prime}, \sigma^{\prime}\right)$ as in [30, Definition 6.4.3.1]. Accordingly (cf. Theorem 12.1), under (13.5), the $\left[\left(\Phi_{\mathcal{H}}, \delta_{\mathcal{H}}\right)\right]$-stratum $\vec{Z}_{\left[\left(\Phi_{\mathcal{H}}, \delta_{\mathcal{H}}\right)\right], \mathrm{J}}$ of $\overrightarrow{\mathrm{M}}_{\mathcal{H}, \mathrm{J}}^{\min }$ is mapped to the $\left[\left(\Phi_{\mathcal{H}^{\prime}}^{\prime}, \delta_{\mathcal{H}^{\prime}}^{\prime}\right)\right]$-stratum $\overrightarrow{\mathrm{Z}}_{\left[\left(\Phi_{\mathcal{H}^{\prime}}^{\prime}, \delta_{\mathcal{H}^{\prime}}^{\prime}\right)\right], \mathrm{J}^{\prime}}$ of $\overrightarrow{\mathrm{M}}_{\mathcal{H}^{\prime}, J^{\prime}}^{\min }$ if and only if there are representatives $\left(\Phi_{\mathcal{H}}, \delta_{\mathcal{H}}\right)$ and $\left(\Phi_{\mathcal{H}^{\prime}}^{\prime}, \delta_{\mathcal{H}^{\prime}}^{\prime}\right)$ of the cusp labels $\left[\left(\Phi_{\mathcal{H}}, \delta_{\mathcal{H}}\right)\right]$ and $\left[\left(\Phi_{\mathcal{H}^{\prime}}^{\prime}, \delta_{\mathcal{H}^{\prime}}^{\prime}\right)\right]$, respectively, such that $\left(\Phi_{\mathcal{H}^{\prime}}^{\prime}, \delta_{\mathcal{H}^{\prime}}^{\prime}\right)$ is 1-assigned to $\left(\Phi_{\mathcal{H}}, \delta_{\mathcal{H}}\right)$ as in [30, Definition 5.4.3.9]. Consequently, the morphism (13.2) is projective, because it is the pullback of the projective morphism (13.5) to the $[(0,0)]$-stratum $\overrightarrow{\mathrm{Z}}_{\left[(0,0], \mathrm{J}^{\prime}\right.}=\overrightarrow{\mathrm{M}}_{\mathcal{H}^{\prime}, \mathrm{J}^{\prime}}$ of $\overrightarrow{\mathrm{M}}_{\mathcal{H}^{\prime}, \mathrm{J}^{\prime}}^{\min }$.

Proof. The statement for the morphism (13.8) follows from the defining property of the strata in Definition 9.1, by comparing the universal properties of $\overrightarrow{\mathrm{M}}_{\mathcal{H}, \Sigma, \mathrm{J}}^{\text {tor }}$ and $\overrightarrow{\mathrm{M}}_{\mathcal{H}^{\prime}, \Sigma^{\prime}, J^{\prime}}^{\text {tor }}$ as in Theorems 7.14 and 11.4. By Theorem 12.1, the statement for 
the morphism (13.5) then follows from the one for (13.8). The statement for the morphism (13.2) is self-explanatory.

REMARK 13.10. Although the morphisms (13.3) and (13.6) in characteristic zero are always finite, the extended morphisms (13.2) and (13.5) in mixed characteristics are projective but not finite in general (even when $\mathcal{H}=\mathcal{H}^{\prime}$ ).

EXAMPLE 13.11 (Simplest case; continuation of Example 2.3). Suppose $J=\left\{\mathrm{j}_{0}\right\}$ and $\left(g_{\mathrm{j}_{0}}, L_{\mathrm{j}_{0}},\langle\cdot, \cdot\rangle_{\mathrm{j}_{0}}\right)=(1, L,\langle\cdot, \cdot\rangle)$ are as in Example 2.3 (we shall suppress the subscript $\mathbf{J}$ for simplicity). Let $\mathcal{H}^{p}$ be the image of $\mathcal{H}$ under the canonical homomorphism $\mathrm{G}(\hat{\mathbb{Z}}) \rightarrow \mathrm{G}\left(\hat{\mathbb{Z}}^{p}\right)$ as usual. Consider the naive moduli problem $\dddot{\mathrm{M}}_{\mathcal{H}^{p}}$ parameterizing tuples $\left(A, \lambda, i, \alpha_{\mathcal{H}^{p}}\right)$ over schemes $S$ over $\operatorname{Spec}\left(\mathcal{O}_{F_{0},(p)}\right)$, where:

(1) $A \rightarrow S$ is an abelian scheme of relative dimension $g:=\frac{1}{2} \operatorname{rk}_{\mathbb{Z}}(L)$.

(2) $\lambda: A \rightarrow A^{\vee}$ is a polarization of degree $d^{2}:=\left[L^{\#}: L\right]$.

(3) $i: \mathcal{O} \hookrightarrow \operatorname{End}_{S}(A)$ is an $\mathcal{O}$-endomorphism structure as in [30, Definition 1.3.3.1].

(4) $\underline{\mathrm{Lie}}_{A / S}$ with its $\mathcal{O} \otimes_{\mathbb{Z}} \mathbb{Z}_{(p)}$-module structure given by $i$ satisfies the determinantal condition in [30, Definition 1.3.4.1] given by $\left(L \otimes_{\mathbb{Z}} \mathbb{R}\right.$, $\left.\langle\cdot, \cdot\rangle, h_{0}\right)$.

(5) $\alpha_{\mathcal{H}^{p}}$ is an (integral) level- $\mathcal{H}^{p}$ structure of $(A, \lambda, i)$ of type $\left(L \otimes_{\mathbb{Z}} \hat{\mathbb{Z}}^{p},\langle\cdot, \cdot\rangle\right)$ as in [30, Definition 1.3.7.6], except that we do not require the degree of $\lambda$ to be prime to $p$ (see [31, Definition 3.3.1.4] for a similar consideration).

We consider this moduli problem naive, because there is no level structure at $p$, and so there is little control on the finite locally free group scheme $\operatorname{ker}(\lambda)\left[p^{\infty}\right]$ beyond its rank. Nevertheless, the canonical morphism $\mathrm{M}_{\mathcal{H}} \rightarrow \mathcal{A}_{g, d} \otimes_{\mathbb{Z}} \mathbb{Z}_{(p)}$ as in the proof of Proposition 6.1 factors as a composition $\mathrm{M}_{\mathcal{H}} \rightarrow \dddot{\mathrm{M}}_{\mathcal{H}^{p}} \rightarrow \mathcal{A}_{g, d} \otimes_{\mathbb{Z}} \mathbb{Z}_{(p)}$ of canonical forgetful morphisms, where the second one is schematic and finite by [30, Proposition 1.3.3.7] (for the endomorphism structures), and by the fact that the level structures (away from $p$ ) are defined by isomorphisms between finite étale group schemes. Therefore, $\overrightarrow{\mathrm{M}}_{\mathcal{H}}$ is canonically isomorphic to the normalization of $\dddot{\mathrm{M}}_{\mathcal{H}^{p}}$ under the canonical morphism $\mathrm{M}_{\mathcal{H}} \rightarrow \dddot{\mathrm{M}}_{\mathcal{H}^{p}}$. Moreover, the tautological tuple $(\vec{A}, \vec{\lambda}, \vec{i})$ over $\overrightarrow{\mathrm{M}}_{\mathcal{H}}$ (see Proposition 6.1 ) is canonically isomorphic to the pullback of the tautological tuple $(A, \lambda, i)$ over $\dddot{\mathrm{M}}_{\mathcal{H}^{p}}$ under the induced morphism $\overrightarrow{\mathrm{M}}_{\mathcal{H}} \rightarrow \dddot{\mathrm{M}}_{\mathcal{H}^{p}}$. If $\mathcal{H}=\mathcal{H}^{p} \mathrm{G}\left(\mathbb{Z}_{p}\right)$, then the canonical morphism 
$\mathrm{M}_{\mathcal{H}} \rightarrow \dddot{\mathrm{M}}_{\mathcal{H}^{p}} \otimes_{\mathbb{Z}_{(p)}} \mathbb{Q}$ is open and closed by the same argument as in the proof of [30, Lemma 1.4.4.2]. In this case, if $\dddot{M}_{\mathcal{H}^{p}}$ is flat over $\operatorname{Spec}\left(\mathbb{Z}_{(p)}\right)$ and normal, or if the schematic closure $\dddot{M}_{\mathcal{H}^{p}}^{+}$of $\dddot{\mathrm{M}}_{\mathcal{H}^{p}} \otimes_{\mathbb{Z}} \mathbb{Q}$ in $\dddot{\mathrm{M}}_{\mathcal{H}^{p}}$ is normal, then the induced morphism $\overrightarrow{\mathrm{M}}_{\mathcal{H}} \rightarrow \dddot{\mathrm{M}}_{\mathcal{H}^{p}}^{+}$is also an open and closed immersion, which implies that $\overrightarrow{\mathrm{M}}_{\mathcal{H}}$ is just the schematic closure of $\mathrm{M}_{\mathcal{H}}$ in $\dddot{\mathrm{M}}_{\mathcal{H}^{p}}$ (or rather $\dddot{\mathrm{M}}_{\mathcal{H}^{p}}^{+}$). In this case, we can deduce the local properties of $\overrightarrow{\mathrm{M}}_{\mathcal{H}}$ (in additional to normality) from those of the local model of $\dddot{\mathrm{M}}_{\mathcal{H}^{p}}^{+}$(for example, see the proofs of Lemmas 14.6 and 14.7 below). Then $\overrightarrow{\mathrm{M}}_{\mathcal{H}}^{\text {min }}$ and $\overrightarrow{\mathrm{M}}_{\mathcal{H}, \Sigma}^{\text {tor }}$ give compactifications of the largest 'relevant' open and closed subscheme of $\dddot{\mathrm{M}}_{\mathcal{H}^{p}}^{+}$, enjoying the various good features we have shown in this article.

EXAMPLE 13.12 (Parahoric levels at $p$; continuation of Example 2.4). Let us resume the context of Example 2.4. For each $0 \leqslant i \leqslant m$, let $\mathbf{J}_{i}:=\left\{\mathrm{j}_{i}\right\}$, and let $\mathcal{H}_{i}:=\mathcal{H}^{p} \mathrm{G}_{\mathrm{j} i}\left(\mathbb{Z}_{p}\right)$. By Propositions 13.1 and 13.9, for each such $i$, we have a canonical projective morphism $\overrightarrow{\mathrm{M}}_{\mathcal{H}, \mathrm{J}} \rightarrow \overrightarrow{\mathrm{M}}_{\mathcal{H}_{i}, \mathrm{~J}_{i}}$ (which is not finite in general). As explained in Example 13.11, the target space $\vec{M}_{\mathcal{H}_{i}, \mathrm{~J}_{i}}$ is canonically isomorphic to the normalization of some naive moduli problem $\ddot{M}_{\mathcal{H}^{p}, \mathrm{~J}_{i}}$ under the canonical morphism $\mathrm{M}_{\mathcal{H}_{i}} \rightarrow \dddot{\mathrm{M}}_{\mathcal{H}^{p}, \mathrm{~J}_{i}}$, and the argument there also implies that $\overrightarrow{\mathrm{M}}_{\mathcal{H}, \mathrm{J}}$ is canonically isomorphic to the normalization of $\prod_{0 \leqslant i \leqslant m} \dddot{\mathrm{M}}_{\mathcal{H}^{p}, \mathrm{~J}_{i}}$ under the canonical morphism $\mathrm{M}_{\mathcal{H}} \rightarrow \prod_{0 \leqslant i \leqslant m} \mathrm{M}_{\mathcal{H}_{i}} \rightarrow \prod_{0 \leqslant i \leqslant m} \dddot{\mathrm{M}}_{\mathcal{H}^{p}, \mathrm{~J}_{i}}$ (we introduce the subscript $\mathrm{J}_{i}$ to emphasize that its definition uses the lattice $L_{\mathrm{j}_{i}}$ ). Therefore, we have canonical finite morphisms $\overrightarrow{\mathrm{M}}_{\mathcal{H}, \mathrm{J}} \rightarrow \prod_{0 \leqslant i \leqslant m} \overrightarrow{\mathrm{M}}_{\mathcal{H}_{\mathrm{j}}, \mathrm{J}_{\mathrm{j}}} \rightarrow \prod_{0 \leqslant i \leqslant m} \dddot{\mathrm{M}}_{\mathcal{H}^{p}, \mathrm{~J}_{i}}$. Moreover, for each $0 \leqslant i \leqslant m$, the tautological tuple $\left(\vec{A}_{\mathrm{j}_{i}}, \vec{\lambda}_{\mathrm{j}_{i}}, \vec{i}_{\mathrm{j}_{i}}\right)$ over $\overrightarrow{\mathrm{M}}_{\mathcal{H}, \mathrm{J}_{i}}$ (see Proposition 6.1) is canonically isomorphic to the pullback of the tautological tuple $\left(A_{\mathrm{j}_{i}}, \lambda_{\mathrm{j}_{i}}, i_{\mathrm{j}_{i}}\right)$ over $\dddot{\mathrm{M}}_{\mathcal{H}^{p}, \mathrm{~J}_{i}}$ under the induced morphism $\overrightarrow{\mathrm{M}}_{\mathcal{H}, \mathrm{J}_{i}} \rightarrow \dddot{\mathrm{M}}_{\mathcal{H}^{p}, \mathrm{~J}_{i}}$, whose further pullback to $\overrightarrow{\mathrm{M}}_{\mathcal{H}, \mathrm{J}}$ is canonically isomorphic to the tautological tuple $\left(\vec{A}_{\mathrm{j}_{i}}, \vec{\lambda}_{\mathrm{j}_{i}}, \vec{i}_{\mathrm{j}_{i}}\right)$ (abusively denoted by the same symbols) over $\overrightarrow{\mathrm{M}}_{\mathcal{H}, \mathrm{J}}$. On the other hand, by considering (periodic) isogeny chains of polarized abelian schemes (with compatible additional structures) as in [51, Definition 6.9], we obtain a naive moduli problem $\dddot{\mathrm{M}}_{\mathcal{H}^{p}, \mathrm{~J}}$, which carries (up to periodicity) a tautological isogeny chain

$$
A=A_{\mathrm{j}_{0}} \rightarrow A_{\mathrm{j}_{1}} \rightarrow \cdots \rightarrow A_{\mathrm{j}_{m}} \rightarrow A
$$

(with compatible additional structures) and admits a canonical forgetful morphism $\ddot{\mathrm{M}}_{\mathcal{H}^{p}, \mathrm{~J}} \rightarrow \dddot{\mathrm{M}}_{\mathcal{H}^{p}, \mathrm{~J}_{i}}$, for each $0 \leqslant i \leqslant m$, under which the tautological $\left(A_{\mathrm{j}_{i}}, \lambda_{\mathrm{j}_{i}}, i_{\mathrm{j}_{i}}\right)$ over $\dddot{\mathrm{M}}_{\mathcal{H}^{p}, \mathrm{~J}}$ is canonically isomorphic to the pullback of the 
tautological $\left(A_{\mathrm{j}_{i}}, \lambda_{\mathrm{j}_{i}}, i_{\mathrm{j}_{i}}\right)$ over $\dddot{\mathrm{M}}_{\mathcal{H}^{p}, \mathrm{~J}_{i}}$. Since $\overrightarrow{\mathrm{M}}_{\mathcal{H}, \mathrm{J}}$ is noetherian normal, the tautological isogeny chain over $\mathrm{M}_{\mathcal{H}}$ (see Example 2.4) canonically extends to an isogeny chain

$$
\vec{A}=\vec{A}_{\mathrm{j}_{0}} \rightarrow \vec{A}_{\mathrm{j}_{1}} \rightarrow \cdots \rightarrow \vec{A}_{\mathrm{j}_{m}} \rightarrow \vec{A}
$$

(with compatible additional structures) over $\overrightarrow{\mathrm{M}}_{\mathcal{H}, \mathrm{J}}$ (see [52, IX, 1.4], [10, Ch. I, Proposition 2.7], or [30, Proposition 3.3.1.5]), where each $\left(\vec{A}_{\mathrm{j}_{i}}, \vec{\lambda}_{\mathrm{j}_{i}}, \vec{i}_{\mathrm{j}_{i}}\right)$ is the tautological one over $\overrightarrow{\mathrm{M}}_{\mathcal{H}, \mathrm{J}}$. By the universal property of $\ddot{\mathrm{M}}_{\mathcal{H}^{p}, \mathrm{~J}}$, we obtain a canonical morphism $\overrightarrow{\mathrm{M}}_{\mathcal{H}, \mathrm{J}} \rightarrow \dddot{\mathrm{M}}_{\mathcal{H}^{p}, \mathrm{~J}}$ under which the above extended isogeny chain (with its compatible additional structures) over $\overrightarrow{\mathrm{M}}_{\mathcal{H}, \mathrm{J}}$ is canonically isomorphic to the pullback of the tautological one over $\dddot{\mathrm{M}}_{\mathcal{H}^{p}, \mathrm{~J}}$. Now, consider the composition $\overrightarrow{\mathrm{M}}_{\mathcal{H}, \mathrm{J}} \rightarrow \dddot{\mathrm{M}}_{\mathcal{H}^{p}, \mathrm{~J}} \rightarrow \prod_{0 \leqslant i \leqslant m} \dddot{\mathrm{M}}_{\mathcal{H}^{p}, \mathrm{~J}_{i}}$ of the morphisms we have just defined. By definition, the tautological tuple $\left(\vec{A}_{\mathrm{j}_{i}}, \vec{\lambda}_{\mathrm{j}_{i}}, \vec{i}_{\mathrm{j}_{i}}\right)$ over $\overrightarrow{\mathrm{M}}_{\mathcal{H}, \mathrm{J}}$ is canonically isomorphic to the pullback of the tautological tuple $\left(A_{\mathrm{j}_{i}}, \lambda_{\mathrm{j}_{i}}, i_{\mathrm{j}_{i}}\right)$ over $\dddot{\mathrm{M}}_{\mathcal{H}^{p}, \mathrm{~J}_{i}}$ under the induced morphism $\overrightarrow{\mathrm{M}}_{\mathcal{H}, \mathrm{J}} \rightarrow \dddot{\mathrm{M}}_{\mathcal{H}^{p}, \mathrm{~J}_{i}}$. Hence, the last composition coincides with the earlier composition $\overrightarrow{\mathrm{M}}_{\mathcal{H}, \mathrm{J}} \rightarrow \prod_{0 \leqslant i \leqslant m} \overrightarrow{\mathrm{M}}_{\mathcal{H}_{\mathrm{j}}, \mathrm{J}_{\mathrm{j}}} \rightarrow \prod_{0 \leqslant i \leqslant m} \dddot{\mathrm{M}}_{\mathcal{H}^{p}, \mathrm{~J}_{i}}$, which is finite (it is crucial that the product runs over all indices $0 \leqslant i \leqslant m$ ). Consequently, the above canonical morphism $\overrightarrow{\mathrm{M}}_{\mathcal{H}, \mathrm{J}} \rightarrow \dddot{\mathrm{M}}_{\mathcal{H}^{p}, \mathrm{~J}}$ is finite, and so $\overrightarrow{\mathrm{M}}_{\mathcal{H}, \mathrm{J}}$ is canonically isomorphic to the normalization of $\dddot{\mathrm{M}}_{\mathcal{H}^{p}, \mathrm{~J}}$ under the canonical morphism $\mathrm{M}_{\mathcal{H}} \rightarrow \ddot{\mathrm{M}}_{\mathcal{H}^{p}, \mathrm{~J}}$. Moreover, since $\mathcal{H}=\mathcal{H}^{p} \mathcal{H}_{p}$, where $\mathcal{H}_{p}=\bigcap_{0 \leqslant i \leqslant m} \mathrm{G}_{\mathrm{j}_{i}}\left(\mathbb{Z}_{p}\right)$, the canonical morphism $\mathrm{M}_{\mathcal{H}} \rightarrow \dddot{\mathrm{M}}_{\mathcal{H}^{p}, \mathrm{~J}} \otimes_{\mathbb{Z}} \mathbb{Q}$ is open and closed by the same argument as in the proof of [30, Lemma 1.4.4.2] (cf. Example 13.11). If $\dddot{\mathrm{M}}_{\mathcal{H}^{p}, \mathrm{~J}}$ is flat over $\operatorname{Spec}\left(\mathbb{Z}_{(p)}\right)$ and normal (see, for example, [12, Theorem 2.1] and [33, Lemma 4.1.18]), or if the schematic closure $\dddot{\mathrm{M}}_{\mathcal{H}^{p}, \mathrm{~J}}^{+}$ of $\dddot{\mathrm{M}}_{\mathcal{H}^{p}, \mathrm{~J}} \otimes_{\mathbb{Z}} \mathbb{Q}$ in $\dddot{\mathrm{M}}_{\mathcal{H}^{p}, \mathrm{~J}}$ is normal (see, for example, [45, Theorem B], [46, Theorem 12.2], and [47, Theorems 1.1 and 1.2]), then the induced morphism $\overrightarrow{\mathrm{M}}_{\mathcal{H}, \mathrm{J}} \rightarrow \dddot{\mathrm{M}}_{\mathcal{H}^{p}, \mathrm{~J}}^{+}$is also an open and closed immersion. In particular, we can deduce the local properties of $\overrightarrow{\mathrm{M}}_{\mathcal{H}, \mathrm{J}}$ (in additional to normality) from those of the local model of $\ddot{M}_{\mathcal{H}^{p}, \mathrm{~J}}^{+}$. Therefore, $\overrightarrow{\mathrm{M}}_{\mathcal{H}, \mathrm{J}}^{\min }$ and $\overrightarrow{\mathrm{M}}_{\mathcal{H}, \Sigma, \mathrm{J}}^{\text {tor }}$ give compactifications of the largest 'relevant' open and closed subscheme of $\ddot{\mathrm{M}}_{\mathcal{H}^{p}, \mathrm{~J}}^{+}$(cf. Example 13.11).

LEMmA 13.13. Given any collection $\left\{\left(g_{\mathrm{j}}, L_{\mathrm{j}},\langle\cdot, \cdot\rangle_{\mathrm{j}}\right)\right\}_{\mathrm{j} \in \mathrm{J}}$ satisfying the conditions imposed by an open compact subgroup $\mathcal{H} \subset \mathrm{G}(\hat{\mathbb{Z}})$ as in Section 2, suppose $g \in \mathrm{G}\left(\mathbb{A}^{\infty}\right)$ satisfies $g^{-1} \mathcal{H} g \subset \mathrm{G}(\hat{\mathbb{Z}})$. Let us formally set $\mathrm{J}^{\prime}:=\mathrm{J}$, so that $\left\{\left(g^{-1} g_{\mathrm{j}}, L_{\mathrm{j}},\langle\cdot, \cdot\rangle_{\mathrm{j}}\right\}_{\mathrm{j} \in \mathrm{J}^{\prime}}\right.$ is a collection with respect to $g^{-1} \mathcal{H} g$ as in Section 2 . Then we have a canonical isomorphism $\overrightarrow{\mathrm{M}}_{\mathcal{H}, \mathrm{J}} \stackrel{\sim}{\rightarrow} \overrightarrow{\mathrm{M}}_{g^{-1} \mathcal{H} g, \mathrm{~J}^{\prime}}$ extending the canonical 
isomorphism $\mathrm{M}_{\mathcal{H}} \stackrel{\sim}{\rightarrow} \mathrm{M}_{g^{-1}}$ Hg realized by sending objects parameterized by $\mathrm{M}_{\mathcal{H}}$ to their Hecke twists by g (see [30, Section 6.4.3]; cf. [30, Proposition 1.4.3.4 and Remark 1.4.3.11]).

Proof. This is because both $\overrightarrow{\mathrm{M}}_{\mathcal{H}, \mathrm{J}}$ and $\overrightarrow{\mathrm{M}}_{g^{-1}} \mathcal{H}_{g, \mathrm{~J}^{\prime}}$ are constructed by normalization using the same collection of auxiliary models $M_{\mathcal{H}_{\mathrm{j}, \text { aux }}}$ for $\mathrm{M}_{\mathcal{H}_{\mathrm{j}}}$, for $\mathrm{j} \in \mathrm{J}=\mathrm{J}^{\prime}$.

EXAMPLE 13.14 (Hecke twists; continuation of Example 2.5). First let us suppose $\mathbf{J}=\left\{\mathrm{j}_{0}, \mathrm{j}_{1}\right\}$ has two elements, with $\left(g_{\mathrm{j}_{0}}, L_{\mathrm{j}_{0}},\langle\cdot, \cdot\rangle_{\mathrm{j}_{0}}\right)=(1, L,\langle\cdot, \cdot\rangle)$ and $\left(g_{\mathrm{j}_{1}}, L_{\mathrm{j}_{1}},\langle\cdot, \cdot\rangle_{\mathrm{j}_{1}}\right)=(g, L,\langle\cdot, \cdot\rangle)$ for some $g \in \mathrm{G}(\hat{\mathbb{Z}})$, as in the first half of Example 2.5. Let $\mathrm{J}_{0}:=\left\{\mathrm{j}_{0}\right\}$ and $\mathrm{J}_{1}:=\left\{\mathrm{j}_{1}\right\}$. Suppose $\mathcal{H}^{\prime} \subset \mathrm{G}(\hat{\mathbb{Z}})$ contains both $\mathcal{H}$ and $g^{-1} \mathcal{H} g$. By Proposition 13.1, we have two canonical morphisms

$$
\overrightarrow{\mathrm{M}}_{\mathcal{H}, \mathrm{J}} \rightarrow \overrightarrow{\mathrm{M}}_{\mathcal{H}, \mathrm{J}_{0}}
$$

and

$$
\overrightarrow{\mathrm{M}}_{\mathcal{H}, \mathrm{J}} \rightarrow \overrightarrow{\mathrm{M}}_{\mathcal{H}, \mathrm{J}_{1}} \cong \overrightarrow{\mathrm{M}}_{g^{-1} \mathcal{H} g, \mathrm{~J}_{0}}
$$

(where the last isomorphism uses Lemma 13.13, since $g^{-1} g_{\mathrm{j}_{1}}=1=g_{\mathrm{j}_{0}}$ ), which induce two canonical morphisms

$$
[\overrightarrow{1}],[\vec{g}]: \overrightarrow{\mathrm{M}}_{\mathcal{H}, \mathrm{J}} \rightarrow \overrightarrow{\mathrm{M}}_{\mathcal{H}^{\prime}, \mathrm{J}_{0}}
$$

extending the two finite morphisms

$$
[1]: \mathrm{M}_{\mathcal{H}} \rightarrow \mathrm{M}_{\mathcal{H}^{\prime}}
$$

and

$$
[g]: \mathrm{M}_{\mathcal{H}} \stackrel{\sim}{\rightarrow} \mathrm{M}_{g^{-1} \mathcal{H g}} \rightarrow \mathrm{M}_{\mathcal{H}^{\prime}}
$$

defining the Hecke correspondence defined by $g$ in characteristic zero. By Proposition 13.9 , the two morphisms $[\overrightarrow{1}]$ and $[\vec{g}]$ are projective, but they are not finite in general (nevertheless, they are finite when $g \in \mathrm{G}\left(\mathbb{A}^{\infty, p}\right) \times \mathrm{G}\left(\mathbb{Z}_{p}\right)$. See Proposition 13.19 below). By Propositions 13.4 and 13.7, they extend to two canonical morphisms

$$
[\overrightarrow{1}]^{\min },[\vec{g}]^{\min }: \overrightarrow{\mathrm{M}}_{\mathcal{H}, \mathrm{J}}^{\min } \rightarrow \overrightarrow{\mathrm{M}}_{\mathcal{H}^{\prime}, \mathrm{J}_{0}}^{\min }
$$

which lift to two canonical morphisms

$$
[\overrightarrow{1}]^{\text {tor }},[\vec{g}]^{\text {tor }}: \overrightarrow{\mathrm{M}}_{\mathcal{H}, \Sigma, \mathrm{J}}^{\text {tor }} \rightarrow \overrightarrow{\mathrm{M}}_{\mathcal{H}^{\prime}, \Sigma^{\prime}, \mathrm{J}_{0}}^{\text {tor }}
$$

(Part of the assertion is that there do exist the compatible choices $\Sigma$ and $\Sigma^{\prime}$ of cone decompositions. This is because we can just take $\Sigma$ to be induced by $\Sigma^{\prime}$ and the twist of $\Sigma^{\prime}$ by $g$. Therefore, up to refinements, we always have enough 
compatible choices of cone decompositions for defining morphisms extending the Hecke correspondence defined by $g$ in characteristic zero.) More generally, for any collection $\left\{\left(g^{\epsilon} g_{\mathrm{j}}, L_{\mathrm{j}},\langle\cdot, \cdot\rangle_{\mathrm{j}}\right)\right\}_{(\epsilon, \mathrm{j}) \in\{0,1\} \times \mathrm{J}^{\prime}}$ attached to a collection $\left\{\left(g_{\mathrm{j}}, L_{\mathrm{j}}\right.\right.$, $\left.\left.\langle\cdot, \cdot\rangle_{\mathrm{j}}\right)\right\}_{\mathrm{j} \in \mathrm{J}^{\prime}}$ as in the second half of Example 2.5, we also have similar morphisms extending the Hecke correspondence defined by $g$ in characteristic zero. Given the importance of these morphisms, let us spell out the precise statements, with some minor modifications of notation:

Proposition 13.15. Given any collection $\left\{\left(g_{\mathrm{j}}, L_{\mathrm{j}},\langle\cdot, \cdot\rangle_{\mathrm{j}}\right)\right\}_{\mathrm{j} \in \mathrm{J}}$ satisfying the conditions imposed by an open compact subgroup $\mathcal{H} \subset \mathrm{G}(\hat{\mathbb{Z}})$ as in Section 2, suppose that $\mathcal{H}^{\prime} \subset \mathrm{G}(\hat{\mathbb{Z}})$ contains both $\mathcal{H}$ and $g^{-1} \mathcal{H} g$, and that $g_{\mathrm{j}}^{-1} \mathcal{H}^{\prime} g_{\mathrm{j}}$ stabilizes $L_{\mathrm{j}} \otimes_{\mathbb{Z}} \hat{\mathbb{Z}}$ for all $\mathrm{j} \in \mathrm{J}$, so that $\left\{\left(g_{\mathrm{j}}, L_{\mathrm{j}},\langle\cdot, \cdot\rangle_{\mathrm{j}}\right)\right\}_{\mathrm{j} \in \mathrm{J}}$ also satisfies the condition imposed by $\mathcal{H}^{\prime}$ as in Section 2. Then the collection $\left\{\left(g^{\epsilon} g_{\mathrm{j}}, L_{\mathrm{j}},\langle\cdot, \cdot\rangle_{\mathrm{j}}\right)\right\}_{(\epsilon, \mathrm{j}) \in\{0,1\} \times \mathrm{J}}$ satisfies the condition imposed by $\mathcal{H}$ as well, and we have two canonical projective morphisms

$$
[\overrightarrow{1}],[\vec{g}]: \vec{M}_{\mathcal{H},\{0,1\} \times \mathrm{J}} \rightarrow \overrightarrow{\mathrm{M}}_{\mathcal{H}^{\prime}, \mathrm{J}}
$$

extending the two canonical finite morphisms

$$
[1]: \mathrm{M}_{\mathcal{H}} \rightarrow \mathrm{M}_{\mathcal{H}^{\prime}}
$$

and

$$
[g]: \mathrm{M}_{\mathcal{H}} \stackrel{\sim}{\rightarrow} \mathrm{M}_{g^{-1} \mathcal{H} g} \rightarrow \mathrm{M}_{\mathcal{H}^{\prime}}
$$

defining the Hecke correspondence defined by $g$ in characteristic zero, which extend to two canonical projective morphisms

$$
[\overrightarrow{1}]^{\min },[\vec{g}]^{\min }: \overrightarrow{\mathrm{M}}_{\mathcal{H},\{0,1\} \times \mathrm{J}}^{\min } \rightarrow \overrightarrow{\mathrm{M}}_{\mathcal{H}^{\prime}, \mathrm{J}}^{\min }
$$

Given any $\Sigma^{\prime}$ such that $\overrightarrow{\mathrm{M}}_{\mathcal{H}^{\prime}, \Sigma^{\prime}, \mathrm{J}}^{\text {tor }}$ is defined, there also exist some $\Sigma$ such that $\overrightarrow{\mathrm{M}}_{\mathcal{H}, \Sigma, \mathrm{J}}^{\mathrm{tor}}$ is defined, and such that the two canonical projective morphisms (13.16) extend to two canonical projective morphisms

$$
[\overrightarrow{1}]^{\text {tor }},[\vec{g}]^{\text {tor }}: \overrightarrow{\mathrm{M}}_{\mathcal{H}, \Sigma,\{0,1\} \times \mathrm{J}}^{\text {tor }} \rightarrow \overrightarrow{\mathrm{M}}_{\mathcal{H}^{\prime}, \Sigma^{\prime}, \mathrm{J}}^{\text {tor }}
$$

compatible with the two canonical projective morphisms (13.17).

The morphism $[\overrightarrow{1}]^{\text {tor }}$ (respectively $[\vec{g}]^{\text {tor }}$ ) in (13.18) maps the $\left[\left(\Phi_{\mathcal{H}}, \delta_{\mathcal{H}}\right.\right.$, $\sigma)]$-stratum $\overrightarrow{\mathrm{Z}}_{\left[\left(\Phi_{\mathcal{H}}, \delta_{\mathcal{H}}, \sigma\right)\right],\{0,1\} \times \mathrm{J}}$ of $\overrightarrow{\mathrm{M}}_{\mathcal{H}, \Sigma,\{0,1\} \times \mathrm{J}}^{\text {tor }}$ to the $\left[\left(\Phi_{\mathcal{H}^{\prime}}^{\prime}, \delta_{\mathcal{H}^{\prime}}, \sigma^{\prime}\right)\right]$-stratum $\vec{Z}_{\left[\left(\Phi_{\mathcal{H}^{\prime}}^{\prime}, \delta_{\mathcal{H}^{\prime}}, \sigma^{\prime}\right)\right], \mathrm{J}}$ of $\overrightarrow{\mathrm{M}}_{\mathcal{H}^{\prime}, \Sigma^{\prime}, \mathrm{J}}^{\text {tor }}$ if and only if there are representatives $\left(\Phi_{\mathcal{H}}, \delta_{\mathcal{H}}, \sigma\right)$ and $\left(\Phi_{\mathcal{H}^{\prime}}^{\prime}, \delta_{\mathcal{H}^{\prime}}^{\prime}, \sigma^{\prime}\right)$ of the equivalence classes $\left[\left(\Phi_{\mathcal{H}}, \delta_{\mathcal{H}}, \sigma\right)\right]$ and $\left[\left(\Phi_{\mathcal{H}^{\prime}}^{\prime}, \delta_{\mathcal{H}^{\prime}}^{\prime}, \sigma^{\prime}\right)\right]$, respectively, such that $\left(\Phi_{\mathcal{H}}, \delta_{\mathcal{H}}, \sigma\right)$ is a 1-refinement (respectively g-refinement) 
of $\left(\Phi_{\mathcal{H}^{\prime}}^{\prime}, \delta_{\mathcal{H}^{\prime}}^{\prime}, \sigma^{\prime}\right)$ as in [30, Definition 6.4.3.1]. Accordingly (cf. Theorem 12.1), the morphism $[1]^{\mathrm{min}}$ (respectively $[g]^{\mathrm{min}}$ ) in (13.17) maps the $\left[\left(\Phi_{\mathcal{H}}, \delta_{\mathcal{H}}\right)\right]$-stratum $\overrightarrow{\mathrm{Z}}_{\left[\left(\Phi_{\mathcal{H}}, \delta_{\mathcal{H}}\right)\right],\{0,1\} \times \mathrm{J}}$ of $\overrightarrow{\mathrm{M}}_{\mathcal{H},\{0,1\} \times \mathrm{J}}^{\min }$ to the $\left[\left(\Phi_{\mathcal{H}^{\prime}}^{\prime}, \delta_{\mathcal{H}^{\prime}}^{\prime}\right)\right]$-stratum $\overrightarrow{\mathrm{Z}}_{\left[\left(\Phi_{\mathcal{H}^{\prime}}^{\prime}, \delta_{\mathcal{H}^{\prime}}^{\prime}\right)\right], \mathrm{J}}$ of $\overrightarrow{\mathrm{M}}_{\mathcal{H}^{\prime}, \mathrm{J}}^{\min }$ if and only if there are representatives $\left(\Phi_{\mathcal{H}}, \delta_{\mathcal{H}}\right)$ and $\left(\Phi_{\mathcal{H}^{\prime}}^{\prime}, \delta_{\mathcal{H}^{\prime}}^{\prime}\right)$ of the cusp labels $\left[\left(\Phi_{\mathcal{H}}, \delta_{\mathcal{H}}\right)\right]$ and $\left[\left(\Phi_{\mathcal{H}^{\prime}}^{\prime}, \delta_{\mathcal{H}^{\prime}}^{\prime}\right)\right]$, respectively, such that $\left(\Phi_{\mathcal{H}^{\prime}}^{\prime}, \delta_{\mathcal{H}^{\prime}}^{\prime}\right)$ is 1 assigned (respectively g-assigned) to $\left(\Phi_{\mathcal{H}}, \delta_{\mathcal{H}}\right)$ as in [30, Definition 5.4.3.9]. Consequently, the morphism [ $\overrightarrow{1}]$ (respectively $[\vec{g}]$ ) in (13.16) is the pullback of the projective morphism $[\overrightarrow{1}]^{\min }$ (respectively $[\vec{g}]^{\mathrm{min}}$ ) in $(13.17)$ to the $[(0,0)]$-stratum $\overrightarrow{\mathrm{Z}}_{[(0,0], \mathrm{J}}=\overrightarrow{\mathrm{M}}_{\mathcal{H}^{\prime}, \mathrm{J}}$ of $\overrightarrow{\mathrm{M}}_{\mathcal{H}^{\prime}, \mathrm{J}}^{\min }$.

Proof. These follow from Propositions 13.1, 13.4, 13.7, and 13.9, by the same arguments as in Example 13.14 (with $\left(\mathbf{J}, \mathbf{J}_{0}\right)$ there replaced with $(\{0,1\} \times \mathbf{J}, \mathbf{J})$ here).

Proposition 13.19. In Proposition 13.15, if $g \in \mathrm{G}\left(\mathbb{A}^{\infty, p}\right) \times\left(\bigcap_{0 \leqslant i \leqslant m} \mathrm{G}_{\mathrm{j}_{i}}\left(\mathbb{Z}_{p}\right)\right)$, then the morphisms in (13.16) and (13.17) are finite.

Proof. Under the morphism $[\overrightarrow{1}]$ (respectively $[\vec{g}]$ ) in (13.16), the pullback of the tautological tuple $\left(\vec{A}_{\mathrm{j}}, \vec{\lambda}_{\mathrm{j}}, \vec{i}_{\mathrm{j}}, \vec{\alpha}_{\mathcal{H}_{\mathrm{j}}}\right)$ over $\overrightarrow{\mathrm{M}}_{\mathcal{H}^{\prime}, \mathrm{J}}$ is canonically isomorphic to the tautological tuples $\left(\vec{A}_{(\epsilon, \mathrm{j})}, \vec{\lambda}_{(\epsilon, \mathrm{j})}, \vec{i}_{(\epsilon, \mathrm{j})}, \vec{\alpha}_{\left.\mathcal{H}_{(\epsilon, \mathrm{j}}\right)}\right)$ over $\overrightarrow{\mathrm{M}}_{\mathcal{H},\{0,1\} \times \mathrm{J}}$, where $\epsilon=0$ (respectively $\epsilon=1$ ), for each $\mathrm{j} \in \mathrm{J}$, and where the level structures are only required to be defined over the characteristic zero fibers. Since $\vec{M}_{\mathcal{H},\{0,1\} \times \mathrm{J}}$ is noetherian normal, for each $\mathrm{j} \in \mathrm{J}$, the $\mathbb{Q}^{\times}$-isogeny

$$
f_{\mathrm{j}}:\left(A_{\mathrm{j}}, \lambda_{\mathrm{j}}, i_{\mathrm{j}}, \alpha_{\mathcal{H}_{\mathrm{j}}}\right)=\left(A_{(0, \mathrm{j})}, \lambda_{(0, \mathrm{j})}, i_{(0, \mathrm{j})}, \alpha_{\mathcal{H}_{(0, \mathrm{j})}}\right) \rightarrow\left(A_{\mathrm{j}}^{\prime}, \lambda_{\mathrm{j}}^{\prime}, i_{\mathrm{j}}^{\prime}, \alpha_{g^{-1} \mathcal{H}_{\mathrm{j}} g}^{\prime}\right)
$$

over $\mathrm{M}_{\mathcal{H}} \cong \mathrm{M}_{\mathcal{H}_{\mathrm{j}}}=\mathrm{M}_{\mathcal{H}_{(0, \mathrm{j})}}$ realizing the Hecke twists of by $g$ (see Example 2.5) canonically extends to a $\mathbb{Q}^{\times}$-isogeny

$$
\begin{aligned}
\vec{f}_{\mathrm{j}}:\left(\vec{A}_{\mathrm{j}}, \vec{\lambda}_{\mathrm{j}}, \vec{i}_{\mathrm{j}}, \vec{\alpha}_{\mathcal{H}_{\mathrm{j}}}\right) & =\left(\vec{A}_{(0, \mathrm{j})}, \vec{\lambda}_{(0, \mathrm{j})}, \vec{i}_{(0, \mathrm{j})}, \vec{\alpha}_{\mathcal{H}_{(0, \mathrm{j})}}\right) \rightarrow\left(\vec{A}_{\mathrm{j}}^{\prime}, \vec{\lambda}_{\mathrm{j}}^{\prime}, \vec{i}_{\mathrm{j}}^{\prime}, \vec{\alpha}_{g^{-1} \mathcal{H}_{\mathrm{j}} g}\right) \\
& =\left(\vec{A}_{(1, \mathrm{j})}, \vec{\lambda}_{(1, \mathrm{j})}, \vec{i}_{(1, \mathrm{j})}, \vec{\alpha}_{\mathcal{H}_{(1, \mathrm{j})}}\right)
\end{aligned}
$$

over $\vec{M}_{\mathcal{H},\{0,1\} \times \mathrm{J}}$ (see [52, IX, 1.4], [10, Ch. I, Proposition 2.7], or [30, Proposition 3.3.1.5]) (so far we have not used the assumption on $g$. In this generality, $\overrightarrow{\mathrm{M}}_{\mathcal{H}, \mathrm{J}}$ carries the degenerating family $\left(\vec{A}_{\mathrm{j}}, \vec{\lambda}_{\mathrm{j}}, \vec{i}_{\mathrm{j}}, \vec{\alpha}_{\mathcal{H}_{\mathrm{j}}}\right)$ of type $\mathrm{M}_{\mathcal{H}_{\mathrm{j}}}$ extending the tautological tuple $\left(A_{\mathrm{j}}, \lambda_{\mathrm{j}}, i_{\mathrm{j}}, \alpha_{\mathcal{H}_{\mathrm{j}}}\right)$ over $\mathrm{M}_{\mathcal{H}} \cong \mathrm{M}_{\mathcal{H}_{\mathrm{j}}}$, for each $\mathrm{j} \in \mathrm{J}$, but not necessarily any extension of the Hecke twist $\left(A_{\mathrm{j}}^{\prime}, \lambda_{\mathrm{j}}^{\prime}, i_{\mathrm{j}}^{\prime}, \alpha_{g^{-1} \mathcal{H}_{\mathrm{j} g} g}^{\prime}\right)$.

For each $\mathrm{j} \in \mathrm{J}$, under the assumption that $g \in \mathrm{G}\left(\mathbb{A}^{\infty, p}\right) \times \mathrm{G}_{\mathrm{j}_{i}}\left(\mathbb{Z}_{p}\right)$, the $\mathbb{Q}^{\times}-$ isogeny $f_{\mathrm{j}}$ is in fact a $\mathbb{Z}_{(p)}^{\times}$-isogeny. Hence, there exists an integer $N_{\mathrm{j}}$ prime to $p$ 
such that $N_{\mathrm{j}} f_{\mathrm{j}}$ is an isogeny prime to $p$. Let us fix the choice of such an $N_{\mathrm{j}}$, and consider $K_{\mathrm{j}}:=\operatorname{ker}\left(N_{\mathrm{j}} f_{\mathrm{j}}\right)$, which is contained in $A_{\mathrm{j}}\left[N_{\mathrm{j}}^{\prime}\right]$ for some integer $N_{\mathrm{j}}^{\prime}$ prime to $p$. Since $\overrightarrow{\mathrm{M}}_{\mathcal{H}, \mathrm{J}}$ is noetherian normal, the schematic closure $\vec{K}_{\mathrm{j}}$ of $K_{\mathrm{j}}$ in the finite étale group scheme $\vec{A}_{\mathrm{j}}\left[N_{\mathrm{j}}^{\prime}\right]$ is also finite étale, and so the isogeny $N_{\mathrm{j}} f_{\mathrm{j}}: A_{\mathrm{j}} \rightarrow A_{\mathrm{j}}^{\prime}$ extends to an isogeny $\vec{A}_{\mathrm{j}} \rightarrow \vec{A}_{\mathrm{j}}^{\prime}:=\vec{A}_{\mathrm{j}} / \vec{K}_{\mathrm{j}}$, whose multiplication by $N_{\mathrm{j}}^{-1}$ defines a $\mathbb{Z}_{(p)}^{\times}$-isogeny $\vec{f}_{\mathrm{j}}: \vec{A}_{\mathrm{j}} \rightarrow \vec{A}_{\mathrm{j}}^{\prime}$ extending $f_{\mathrm{j}}$. Moreover, $\vec{A}_{\mathrm{j}}^{\prime}$ is equipped with the additional structures $\vec{\lambda}_{\mathrm{j}}^{\prime}, \vec{i}_{\mathrm{j}}^{\prime}$, and $\vec{\alpha}_{g^{-1} \mathcal{H}_{\mathrm{j} g}}$ over $\overrightarrow{\mathrm{M}}_{\mathcal{H}, \mathrm{J}}$ (see [52, IX, 1.4], [10, Ch. I, Proposition 2.7], or [30, Proposition 3.3.1.5]), where the level structure $\vec{\alpha}_{g^{-1}} \mathcal{H}_{\mathrm{j} g}$ is only required to be defined over the characteristic zero fiber. By the construction of $\overrightarrow{\mathrm{M}}_{\mathcal{H},\{1\} \times \mathrm{J}}$, there is a canonical morphism

$$
s: \overrightarrow{\mathrm{M}}_{\mathcal{H}, \mathrm{J}}=\overrightarrow{\mathrm{M}}_{\mathcal{H},\{0\} \times \mathrm{J}} \rightarrow \overrightarrow{\mathrm{M}}_{\mathcal{H},\{1\} \times \mathrm{J}}
$$

such that the tuple $\left(\vec{A}_{\mathrm{j}}^{\prime}, \vec{\lambda}_{\mathrm{j}}^{\prime}, \vec{i}_{\mathrm{j}}^{\prime}, \vec{\alpha}_{g^{-1} \mathcal{H}_{\mathrm{j} g}}\right)$ over $\overrightarrow{\mathrm{M}}_{\mathcal{H}, \mathrm{J}}$ is canonically isomorphic to the pullback of $\left(\vec{A}_{(1, \mathrm{j})}, \vec{\lambda}_{(1, \mathrm{j})}, \vec{i}_{(1, \mathrm{j})}, \vec{\alpha}_{\mathcal{H}_{(1, j)}}\right)$ over $\overrightarrow{\mathrm{M}}_{\mathcal{H},\{1\} \times \mathrm{J}}$. Since the induced morphism (Id, $s$ ) : $\overrightarrow{\mathrm{M}}_{\mathcal{H}, \mathrm{J}}=\overrightarrow{\mathrm{M}}_{\mathcal{H},\{0\} \times \mathrm{J}} \rightarrow \overrightarrow{\mathrm{M}}_{\mathcal{H},\{0\} \times \mathrm{J}} \times \overrightarrow{\mathrm{M}}_{\mathcal{H},\{1\} \times \mathrm{J}}$ is trivially finite, $\overrightarrow{\mathrm{M}}_{\mathcal{H}, \mathrm{J}}$ is canonically isomorphic to the normalization of $\overrightarrow{\mathrm{M}}_{\mathcal{H},\{0\} \times \mathrm{J}} \times \overrightarrow{\mathrm{M}}_{\mathcal{H},\{1\} \times \mathrm{J}}$ under the canonical morphism $\mathrm{M}_{\mathcal{H}} \rightarrow \overrightarrow{\mathrm{M}}_{\mathcal{H},\{0\} \times \mathrm{J}} \times \overrightarrow{\mathrm{M}}_{\mathcal{H},\{1\} \times \mathrm{J}}$, and hence the canonical morphism $\overrightarrow{\mathrm{M}}_{\mathcal{H},\{0,1\} \times \mathrm{J}} \rightarrow \overrightarrow{\mathrm{M}}_{\mathcal{H}, \mathrm{J}}$ is an isomorphism. Therefore, the morphism [1] : $\overrightarrow{\mathrm{M}}_{\mathcal{H},\{0,1\} \times \mathrm{J}} \rightarrow \overrightarrow{\mathrm{M}}_{\mathcal{H}^{\prime}, \mathrm{J}}$ in (13.16) is finite, because it is the composition of the canonical isomorphism $\overrightarrow{\mathrm{M}}_{\mathcal{H},\{0,1\} \times \mathrm{J}} \rightarrow \overrightarrow{\mathrm{M}}_{\mathcal{H}, \mathrm{J}}$ with the canonical finite morphism $\overrightarrow{\mathrm{M}}_{\mathcal{H}, \mathrm{J}} \rightarrow \overrightarrow{\mathrm{M}}_{\mathcal{H}^{\prime}, \mathrm{J}}$. A similar argument also shows that the morphism $[\vec{g}]: \vec{M}_{\mathcal{H},\{0,1\} \times \mathrm{J}} \rightarrow \overrightarrow{\mathrm{M}}_{\mathcal{H}^{\prime}, \mathrm{J}}$ in (13.16) is finite.

For each $\mathrm{j} \in \mathrm{J}$, consider the degenerating family $\left(\vec{G}_{\mathrm{j}}, \vec{\lambda}_{\mathrm{j}}, \vec{i}_{\mathrm{j}}, \vec{\alpha}_{\mathcal{H}_{\mathrm{j}}}\right)$ over $\overrightarrow{\mathrm{M}}_{\mathcal{H}, \Sigma, \mathrm{J}}^{\text {tor }}$ (see Theorem 11.2), which extends the tautological tuple $\left(\vec{A}_{\mathrm{j}}, \vec{\lambda}_{\mathrm{j}}, \vec{i}_{\mathrm{j}}, \vec{\alpha}_{\mathcal{H}_{\mathrm{j}}}\right)$ over $\overrightarrow{\mathrm{M}}_{\mathcal{H}, \mathrm{J}}$, where $\Sigma$ denotes (temporarily) any compatible collection of cone decompositions that is a 1-refinement of $\Sigma^{\prime}$. Since $g \in \mathrm{G}\left(\mathbb{A}^{\infty, p}\right) \times \mathrm{G}_{\mathrm{j}_{i}}\left(\mathbb{Z}_{p}\right)$, by essentially the same argument as above, the $\mathbb{Z}_{(p)}^{\times}$-isogeny

$$
\vec{f}_{\mathrm{j}}:\left(\vec{A}_{\mathrm{j}}, \vec{\lambda}_{\mathrm{j}}, \vec{i}_{\mathrm{j}}, \vec{\alpha}_{\mathrm{j}}\right) \rightarrow\left(\vec{A}_{\mathrm{j}}^{\prime}, \vec{\lambda}_{\mathrm{j}}^{\prime}, \vec{i}_{\mathrm{j}}^{\prime}, \vec{\alpha}_{g^{-1}} \mathcal{H}_{\mathrm{j}} g\right)
$$

over $\overrightarrow{\mathrm{M}}_{\mathcal{H}, \mathrm{J}}$ extends to a $\mathbb{Z}_{(p)}^{\times}$-isogeny

$$
\vec{f}_{\mathrm{j}}^{\text {tor }}:\left(\vec{G}_{\mathrm{j}}, \vec{\lambda}_{\mathrm{j}}, \vec{i}_{\mathrm{j}}, \vec{\alpha}_{\mathrm{j}}\right) \rightarrow\left(\vec{G}_{\mathrm{j}}^{\prime}, \vec{\lambda}_{\mathrm{j}}^{\prime}, \vec{i}_{\mathrm{j}}^{\prime}, \vec{\alpha}_{g^{-1} \mathcal{H}_{\mathrm{j}} g}\right)
$$

over $\overrightarrow{\mathrm{M}}_{\mathcal{H}, \Sigma, \mathrm{J}}^{\text {tor }}$. By Theorem 11.4, up to replacing $\Sigma$ with a further refinement, there exists a canonical morphism

$$
s^{\text {tor }}: \overrightarrow{\mathrm{M}}_{\mathcal{H}, \Sigma, \mathrm{J}}^{\text {tor }}=\overrightarrow{\mathrm{M}}_{\mathcal{H}, \Sigma,\{0\} \times \mathrm{J}}^{\text {tor }} \rightarrow \overrightarrow{\mathrm{M}}_{\mathcal{H}, \Sigma,\{1\} \times \mathrm{J}}^{\text {tor }}
$$


(extending the morphism $s$ above) such that the tuple $\left(\vec{G}_{\mathrm{j}}^{\prime}, \vec{\lambda}_{\mathrm{j}}^{\prime}, \vec{i}_{\mathrm{j}}^{\prime}, \vec{\alpha}_{g^{-1} \mathcal{H}_{\mathrm{j} g}}\right)$ over $\overrightarrow{\mathrm{M}}_{\mathcal{H}, \Sigma, \mathrm{J}}^{\text {tor }}$ is canonically isomorphic to the pullback of the tautological degenerating family $\left(\vec{G}_{(1, \mathrm{j})}, \vec{\lambda}_{(1, \mathrm{j})}, \vec{i}_{(1, \mathrm{j})}, \vec{\alpha}_{\left.\mathcal{H}_{(1, \mathrm{j})}\right)}\right)$ over $\overrightarrow{\mathrm{M}}_{\mathcal{H},\{1\} \times \mathrm{J}}^{\text {tor }}$. This morphism $s^{\text {tor }}$ then induces a canonical morphism

$$
s^{\min }: \overrightarrow{\mathrm{M}}_{\mathcal{H}, \mathrm{J}}^{\min }=\overrightarrow{\mathrm{M}}_{\mathcal{H},\{0\} \times \mathrm{J}}^{\min } \rightarrow \overrightarrow{\mathrm{M}}_{\mathcal{H},\{1\} \times \mathrm{J}}^{\min }
$$

(also extending the morphism $s$ above). Hence, by essentially the same normalization argument as before, the canonical morphism $\overrightarrow{\mathrm{M}}_{\mathcal{H},\{0,1\} \times \mathrm{J}}^{\min } \rightarrow \overrightarrow{\mathrm{M}}_{\mathcal{H}, \mathrm{J}}^{\min }$ is an isomorphism, and the morphism [ $[\overrightarrow{1}]^{\text {min }}$ in (13.17) is finite. A similar argument also shows that the morphism $[\vec{g}]^{\min }: \overrightarrow{\mathrm{M}}_{\mathcal{H},\{0,1\} \times \mathrm{J}}^{\min } \rightarrow \overrightarrow{\mathrm{M}}_{\mathcal{H}^{\prime}, \mathrm{J}}^{\min }$ in (13.17) is finite.

\section{Local properties}

Proposition 14.1. Suppose $\Sigma$ is smooth as in [30, Definition 6.3.3.4]. Then $\overrightarrow{\mathrm{M}}_{\mathcal{H}}$ is regular if and only if $\overrightarrow{\mathrm{M}}_{\mathcal{H}, \Sigma}^{\text {tor }}$ is.

Proof. Suppose $\sigma \in \Sigma_{\Phi_{\mathcal{H}}}$ and $\sigma \subset \mathbf{P}_{\Phi_{\mathcal{H}}}^{+}$for some representative $\left(\mathrm{Z}_{\mathcal{H}}, \Phi_{\mathcal{H}}\right.$, $\left.\delta_{\mathcal{H}}\right)$ of cusp label. Since $\overrightarrow{\mathrm{M}}_{\mathcal{H}, \Sigma}^{\text {tor }}$ and $\vec{\Xi}_{\Phi_{\mathcal{H}}, \delta_{\mathcal{H}}}(\sigma)$ are excellent, by Theorems 9.13 and 10.13 , by Corollary 10.16, and by [14, IV-2, 7.8.3.1], it suffices to show that, if $\sigma$ is smooth, then $\overrightarrow{\vec{\Xi}}_{\Phi_{\mathcal{H}}, \delta_{\mathcal{H}}}(\sigma)$ is regular if and only if $\vec{\Xi}_{\Phi_{\mathcal{H}}, \delta_{\mathcal{H}}}$ is. By Proposition 8.14, under the assumption that $\sigma$ is smooth, $\vec{\Xi}_{\Phi_{\mathcal{H}}, \delta_{\mathcal{H}}}(\sigma) \rightarrow \vec{C}_{\Phi_{\mathcal{H}}, \delta_{\mathcal{H}}}$ is smooth and surjective, which is faithfully flat and has geometrically regular fibers by definition. In this case, by [35, 21.D], the open subscheme $\vec{\Xi}_{\Phi_{\mathcal{H}}, \delta_{\mathcal{H}}}$ of $\vec{\Xi}_{\Phi_{\mathcal{H}}, \delta_{\mathcal{H}}}(\sigma)$ is regular if and only if the base scheme $\vec{C}_{\Phi_{\mathcal{H}}, \delta_{\mathcal{H}}}$ is, and if and only if the whole scheme $\vec{\Xi}_{\Phi_{\mathcal{H}}, \delta_{\mathcal{H}}}(\sigma)$ is.

The argument above can be slightly improved and show much more:

PROPOSITION 14.2. Let $\mathrm{P}$ be the property of being one of the following: reduced, geometrically reduced, normal, geometrically normal, Cohen-Macaulay, $\left(R_{0}\right)$, geometric $\left(R_{0}\right)$, $\left(R_{1}\right)$, geometric $\left(R_{1}\right)$, and $\left(S_{i}\right)$, one property for each $i \geqslant 0$ (see [14, IV-2, 5.7.2 and 5.8.2]). Then the fiber of $\overrightarrow{\mathrm{M}}_{\mathcal{H}, \Sigma}^{\text {tor }} \rightarrow \overrightarrow{\mathrm{S}}_{0}=\operatorname{Spec}\left(\mathcal{O}_{F_{0},(p)}\right)$ over some point s of $\overrightarrow{\mathrm{S}}_{0}$ satisfies property $\mathrm{P}$ if and only if the corresponding fiber of the open subscheme $\overrightarrow{\mathrm{M}}_{\mathcal{H}} \rightarrow \overrightarrow{\mathrm{S}}_{0}$ over $s$ does. If $\Sigma$ is smooth as in [30, Definition 6.3.3.4], then $\mathrm{P}$ can also be the property of being one of the following: regular, geometrically regular, $\left(R_{i}\right)$, and geometrically $\left(R_{i}\right)$, one property for each $i \geqslant 0$. 
Proof. Suppose $\sigma \in \Sigma_{\Phi_{\mathcal{H}}}$ and $\sigma \subset \mathbf{P}_{\Phi_{\mathcal{H}}}^{+}$for some representative $\left(\mathrm{Z}_{\mathcal{H}}, \Phi_{\mathcal{H}}\right.$, $\left.\delta_{\mathcal{H}}\right)$ of cusp label. Since $\overrightarrow{\mathrm{M}}_{\mathcal{H}, \Sigma}^{\text {tor }}$ and $\vec{\Xi}_{\Phi_{\mathcal{H}}, \delta_{\mathcal{H}}}(\sigma)$ are excellent, by Theorems 9.13 and 10.13, by Corollary 10.16, and by [14, IV-2, 7.8.3.1], it suffices to show that the fiber of $\vec{\Xi}_{\Phi_{\mathcal{H}}, \delta_{\mathcal{H}}} \rightarrow \overrightarrow{\mathrm{S}}_{0}$ above $s$ satisfies property $\mathrm{P}$ if and only if the corresponding fiber of $\vec{\Xi}_{\Phi_{\mathcal{H}}, \delta_{\mathcal{H}}}(\sigma) \rightarrow \overrightarrow{\mathrm{S}}_{0}$ above $s$ does. By [35, 21.C, 21.D, and 21.E], since the torus torsor $\vec{\Xi}_{\Phi_{\mathcal{H}}, \delta_{\mathcal{H}}} \rightarrow \vec{C}_{\Phi_{\mathcal{H}}, \delta_{\mathcal{H}}}$ is smooth and surjective, and since $\vec{\Xi}_{\Phi_{\mathcal{H}}, \delta_{\mathcal{H}}}(\sigma) \rightarrow \vec{C}_{\Phi_{\mathcal{H}}, \delta_{\mathcal{H}}}$ is flat and has geometrically normal and Cohen-Macaulay fibers by Proposition 8.14, which is smooth when $\Sigma$ (or rather $\sigma$ ) is smooth, the fiber of $\vec{\Xi}_{\Phi_{\mathcal{H}}, \delta_{\mathcal{H}}} \rightarrow \overrightarrow{\mathrm{S}}_{0}$ above $s$ satisfies property $\mathrm{P}$ if and only if the fiber of $\vec{C}_{\Phi_{\mathcal{H}}, \delta_{\mathcal{H}}} \rightarrow \overrightarrow{\mathrm{S}}_{0}$ above $s$ does, and so if and only if the fiber of $\vec{\Xi}_{\Phi_{\mathcal{H}}, \delta_{\mathcal{H}}}(\sigma) \rightarrow \overrightarrow{\mathrm{S}}_{0}$ over $s$ also does, as desired.

REMARK 14.3. By [35, 21.C], since $\overrightarrow{\mathrm{M}}_{\mathcal{H}}$ is noetherian and since $\overrightarrow{\mathrm{S}}_{0}=$ $\operatorname{Spec}\left(\mathcal{O}_{F_{0},(p)}\right)$ is Cohen-Macaulay, the flat morphism $\overrightarrow{\mathrm{M}}_{\mathcal{H}} \rightarrow \overrightarrow{\mathrm{S}}_{0}$ has CohenMacaulay fibers if and only if the scheme $\overrightarrow{\mathrm{M}}_{\mathcal{H}}$ is Cohen-Macaulay.

COROLlary 14.4. Suppose that $\overrightarrow{\mathrm{M}}_{\mathcal{H}} \rightarrow \overrightarrow{\mathrm{S}}_{0}$ has geometrically normal fibers. Then all geometric fibers of $\overrightarrow{\mathrm{M}}_{\mathcal{H}, \Sigma}^{\text {tor }} \rightarrow \overrightarrow{\mathrm{S}}_{0}$ have the same number of connected components, and the same is true for $\overrightarrow{\mathrm{M}}_{\mathcal{H}} \rightarrow \overrightarrow{\mathrm{S}}_{0}$ and $\overrightarrow{\mathrm{M}}_{\mathcal{H}}^{\text {min }} \rightarrow \overrightarrow{\mathrm{S}}_{0}$ (the analogous statements are true if we consider irreducible components instead of connected components).

Proof. By the analog of Zariski's connectedness theorem in [8, Theorem 4.17], the assertions for $\overrightarrow{\mathrm{M}}_{\mathcal{H}, \Sigma}^{\text {tor }}$ follow from Lemma 7.9 and Proposition 14.2. Then the assertions for $\vec{M}_{\mathcal{H}}$ follow by Corollary 10.18. The assertions for $\vec{M}_{\mathcal{H}}^{\min }$ follow from those for $\overrightarrow{\mathrm{M}}_{\mathcal{H}, \Sigma}^{\text {tor }}$ and $\overrightarrow{\mathrm{M}}_{\mathcal{H}}$, and from Lemma 12.9, and Corollaries 10.18 and 12.5.

Let us record some examples where $\overrightarrow{\mathrm{M}}_{\mathcal{H}} \rightarrow \overrightarrow{\mathrm{S}}_{0}$ has geometrically normal and Cohen-Macaulay fibers. For these examples, we shall take $\mathrm{J}=\left\{\mathrm{j}_{0}\right\}$ and $\left(g_{\mathrm{j}_{0}}, L_{\mathrm{j}_{0}}\right.$, $\left.\langle\cdot, \cdot\rangle_{\mathrm{j}_{0}}\right)=(1, L,\langle\cdot, \cdot\rangle)$ as in Example 2.3. As a sanity check, let us begin with the following.

REMARK 14.5. Suppose $p$ is a good prime for $\left(\mathcal{O}, \star, L,\langle\cdot, \cdot\rangle, h_{0}\right)$ as in [30, Definition 1.4.1.1]. Then Zarhin's trick is not needed because $p \nmid\left[L^{\#}: L\right]$. Suppose $\mathcal{H}=\mathcal{H}^{p} \mathcal{H}_{p}$ with $\mathcal{H}^{p} \subset \mathrm{G}\left(\hat{\mathbb{Z}}^{p}\right)$ and $\mathcal{H}_{p}=\mathrm{G}\left(\mathbb{Z}_{p}\right)$. Then the moduli problem $\dddot{\mathrm{M}}_{\mathcal{H}^{p}}$ in Example 13.11 is no longer naive, and gives the same $\mathrm{M}_{\mathcal{H}^{p}}$ 
as in [30, Definition 1.4.1.4]. By [30, Lemma 1.4.4.2], we have an open and closed immersion $\overrightarrow{\mathrm{M}}_{\mathcal{H}} \rightarrow \mathrm{M}_{\mathcal{H}^{p}}$, and it follows from the smoothness of $\mathrm{M}_{\mathcal{H}^{p}} \rightarrow$ $\overrightarrow{\mathrm{S}}_{0}$ that $\overrightarrow{\mathrm{M}}_{\mathcal{H}} \rightarrow \overrightarrow{\mathrm{S}}_{0}$ is also smooth, and hence satisfies all the properties in Proposition 14.2.

We will need some deep inputs from the theory of local models in the following nontrivial examples. The following is an important special case considered in, for example, [19] (the actual assumptions there are more restrictive):

LEMMA 14.6. Suppose $\mathcal{H}=\mathcal{H}^{p} \mathcal{H}_{p}$ with $\mathcal{H}^{p} \subset \mathrm{G}\left(\hat{\mathbb{Z}}^{p}\right)$ and $\mathcal{H}_{p}=\mathrm{G}\left(\mathbb{Z}_{p}\right)$. Then $\overrightarrow{\mathrm{M}}_{\mathcal{H}} \rightarrow \overrightarrow{\mathrm{S}}_{0}$ has geometrically normal and Cohen-Macaulay fibers if the integral PEL datum $\left(\mathcal{O}, \star, L,\langle\cdot, \cdot\rangle, h_{0}\right)$ is defined as follows, and if $\mathrm{J}=\left\{\mathrm{j}_{0}\right\}$ and $\left(g_{\mathrm{j}_{0}}, L_{\mathrm{j}_{0}}\right.$, $\left.\langle\cdot, \cdot\rangle_{\mathrm{j}_{0}}\right)=(1, L,\langle\cdot, \cdot\rangle)$ are as in Example 2.3:

(1) $\mathcal{O}=\mathcal{O}_{F}$ is the maximal order given by the ring of integers in a CM number field $F$, with totally real subfield $F^{+}$and with the nontrivial involution * induced by the complex conjugation, such that every prime of $F^{+}$above $p$ is unramified in $F$ (but p may still be arbitrarily ramified in $F^{+}$).

(2) $L=\left(\operatorname{Diff}_{\mathcal{O} / \mathbb{Z}}^{-1}(1)\right)^{\oplus n} \oplus \mathcal{O}^{\oplus n}$ for some integer $n \geqslant 0$.

(3) $\langle\cdot, \cdot\rangle: L \times L \rightarrow \mathbb{Z}(1)$ is the self-dual pairing defined by the composition of the trace map $\operatorname{Tr}_{\mathcal{O} / \mathbb{Z}}: \operatorname{Diff}_{\mathcal{O} / \mathbb{Z}}^{-1}(1) \rightarrow \mathbb{Z}(1)$ with the skew-Hermitian pairing $L \times L \rightarrow \operatorname{Diff}_{\mathcal{O} / \mathbb{Z}}^{-1}(1)$ defined by the matrix $\left(\begin{array}{cc}0 & \mathrm{Id}_{n} \\ -\mathrm{Id}_{n} & 0\end{array}\right)$.

(4) $h_{0}: \mathbb{C} \rightarrow \operatorname{End}_{\mathcal{O} \otimes_{\mathbb{Z}} \mathbb{R}}\left(L \otimes_{\mathbb{Z}} \mathbb{R}\right)$ is defined by

$z=z_{1}+\sqrt{-1} z_{2} \mapsto h_{0}(z):=\left(\begin{array}{cc}z_{1} \operatorname{Id}_{n} & -z_{2}\left((2 \pi \sqrt{-1}) \circ \operatorname{Id}_{n}\right) \\ z_{2}\left(\operatorname{Id}_{n} \circ(2 \pi \sqrt{-1})^{-1}\right) & z_{1} \operatorname{Id}_{n}\end{array}\right)$,

where $2 \pi \sqrt{-1}: \mathbb{Z} \stackrel{\sim}{\rightarrow} \mathbb{Z}(1)$ and $(2 \pi \sqrt{-1})^{-1}: \mathbb{Z}(1) \stackrel{\sim}{\rightarrow} \mathbb{Z}$ stand for the isomorphisms defined by the choice of $\sqrt{-1}$ in $\mathbb{C}$, and where the matrix acts (symbolically) on elements $\left(\begin{array}{l}x \\ y\end{array}\right)$ of $L \otimes_{\mathbb{Z}} \mathbb{R}$ by left multiplication.

Proof. As explained in Example 13.11, by the construction of $\overrightarrow{\mathrm{M}}_{\mathcal{H}}$, it admits an open and closed immersion to the (schematic) closure of the characteristic zero fiber in a naive moduli problem, as soon as this closure is known to be normal. Since the base changes of $\mathrm{G}$ and $\left(\operatorname{Res}_{\mathcal{O}_{F^{+}} / \mathbb{Z}} \mathrm{GL}_{2 n}\right) \times \mathbf{G}_{\mathrm{m}}$ from $\mathbb{Z}$ to the ring $W\left(\overline{\mathbb{F}}_{p}\right)$ of Witt vectors are isomorphic to each other, by the theory of local models (see [51, pages 88-95]), it follows from [45, Theorem B] that $\vec{M}_{\mathcal{H}}$ is normal and that the fibers of $\overrightarrow{\mathrm{M}}_{\mathcal{H}} \rightarrow \overrightarrow{\mathrm{S}}_{0}$ are geometrically normal and Cohen-Macaulay, as desired. 
LEMma 14.7. Suppose $\mathcal{H}=\mathcal{H}^{p} \mathcal{H}_{p}$ with $\mathcal{H}^{p} \subset \mathrm{G}\left(\hat{\mathbb{Z}}^{p}\right)$ and $\mathcal{H}_{p}=\mathrm{G}\left(\mathbb{Z}_{p}\right)$. Then $\overrightarrow{\mathrm{M}}_{\mathcal{H}} \rightarrow \overrightarrow{\mathrm{S}}_{0}$ has geometrically normal and Cohen-Macaulay fibers if the integral PEL datum $\left(\mathcal{O}, \star, L,\langle\cdot, \cdot\rangle, h_{0}\right)$ is defined as follows, and if $\mathbf{J}=\left\{\mathrm{j}_{0}\right\}$ and $\left(g_{\mathrm{j}_{0}}, L_{\mathrm{j}_{0}}\right.$, $\left.\langle\cdot, \cdot\rangle_{\mathrm{j}_{0}}\right)=(1, L,\langle\cdot, \cdot\rangle)$ are as in Example 2.3:

(1) $\mathcal{O}=\mathcal{O}_{F}$ is the maximal order given by the ring of integers in a totally real number field $F$, with the trivial involution *.

(2) $L,\langle\cdot, \cdot\rangle$, and $h_{0}$ are defined as in Lemma 14.6.

Proof. Since base changes of $\mathrm{G}$ and $\operatorname{Res}_{\mathcal{O}_{F} / \mathbb{Z}} \mathrm{GSp}_{2 n}$ from $\mathbb{Z}$ to the ring $W\left(\overline{\mathbb{F}}_{p}\right)$ of Witt vectors are isomorphic to each other up to center, by the same argument as in the proof of Lemma 14.6, and by the theory of local models (see [51, pages 88-95]), it follows from [46, Theorem 12.2] that $\overrightarrow{\mathrm{M}}_{\mathcal{H}}$ is normal and that the fibers of $\overrightarrow{\mathrm{M}}_{\mathcal{H}} \rightarrow \overrightarrow{\mathrm{S}}_{0}$ are geometrically normal and Cohen-Macaulay, as desired.

\section{Density of ordinary loci}

In this section, we will need some input from [31] (we will freely cite definitions and basic results in [31] without repeating them in detail). We shall assume that $\mathbf{J}=\left\{\mathrm{j}_{0}\right\}$ and $\left(g_{\mathrm{j}_{0}}, L_{\mathrm{j}_{0}},\langle\cdot, \cdot\rangle_{\mathrm{j}_{0}}\right)=(1, L,\langle\cdot, \cdot\rangle)$ as in Example 2.3. In this case, the definitions of $\overrightarrow{\mathrm{M}}_{\mathcal{H}}, \overrightarrow{\mathrm{M}}_{\mathcal{H}}^{\text {min }}$, and so on in this article agree with those in [31, in particular Section 2.2.1]. Let $\left(\overrightarrow{\mathrm{M}}_{\mathcal{H}}^{\min } \otimes_{\mathbb{Z}} \mathbb{F}_{p}\right)^{\text {full-ord }}$ and $\left(\overrightarrow{\mathrm{M}}_{\mathcal{H}} \otimes_{\mathbb{Z}} \mathbb{F}_{p}\right)^{\text {full-ord }}$ be defined as in [31, Section 6.3.2].

Proposition 15.1. Suppose that there exists a fully symplectic admissible filtration $\mathrm{Z}$ on $L \otimes_{\mathbb{Z}} \hat{\mathbb{Z}}$ with respect to $(L,\langle\cdot, \cdot\rangle)$ such that $\mathrm{Gr}_{-1}^{\mathrm{Z}}=\mathrm{Z}_{-1} / \mathrm{Z}_{-2}=\{0\}$; that the morphism $\overrightarrow{\mathrm{M}}_{\mathcal{H}^{p} \mathrm{G}\left(\mathbb{Z}_{p}\right)} \rightarrow \overrightarrow{\mathrm{S}}_{0}$ has geometrically normal fibers; and that the algebra $\mathcal{O} \otimes_{\mathbb{Z}} \mathbb{Q}$ involves no simple factor of type $\mathrm{D}$ as in [30, Definition 1.2.1.15]. Then $\left(\overrightarrow{\mathrm{M}}_{\mathcal{H}}^{\min } \otimes_{\mathbb{Z}} \mathbb{F}_{p}\right)^{\text {full-ord }}$ and $\left(\overrightarrow{\mathrm{M}}_{\mathcal{H}} \otimes_{\mathbb{Z}} \mathbb{F}_{p}\right)^{\text {full-ord }}$ are nonempty and dense in $\overrightarrow{\mathrm{M}}_{\mathcal{H}}^{\min } \otimes_{\mathbb{Z}} \mathbb{F}_{p}$ and $\overrightarrow{\mathrm{M}}_{\mathcal{H}} \otimes_{\mathbb{Z}} \mathbb{F}_{p}$, respectively.

Proof. Without loss of generality, we may replace $\mathcal{H}$ with $\mathcal{H}^{p} \mathrm{G}\left(\mathbb{Z}_{p}\right)$ and assume that $\overrightarrow{\mathrm{M}}_{\mathcal{H}} \rightarrow \overrightarrow{\mathrm{S}}_{0}$ has geometrically normal fibers, because the nonemptiness and density assertions are preserved under the finite morphism $\overrightarrow{\mathrm{M}}_{\mathcal{H}} \rightarrow \overrightarrow{\mathrm{M}}_{\mathcal{H}^{p} \mathrm{G}\left(\mathbb{Z}_{p}\right)}$ in this case (for the density assertion, see, for example, [35, 5.E, Theorem 5v)]). Moreover, by Corollary 12.5 , the assertion for $\left(\overrightarrow{\mathrm{M}}_{\mathcal{H}} \otimes_{\mathbb{Z}} \mathbb{F}_{p}\right)^{\text {full-ord }}$ and $\overrightarrow{\mathrm{M}}_{\mathcal{H}} \otimes_{\mathbb{Z}} \mathbb{F}_{p}$ follows form the one for $\left(\overrightarrow{\mathrm{M}}_{\mathcal{H}}^{\min } \otimes_{\mathbb{Z}} \mathbb{F}_{p}\right)^{\text {full-ord }}$ and $\overrightarrow{\mathrm{M}}_{\mathcal{H}}^{\min } \otimes_{\mathbb{Z}} \mathbb{F}_{p}$, and so it suffices to prove the latter. 
Consider any $\overrightarrow{\mathrm{M}}_{\mathcal{H}, \Sigma}^{\text {tor }}$ as in Section 7, and let $\left(\overrightarrow{\mathrm{M}}_{\mathcal{H}, \Sigma}^{\text {tor }}\right)^{\text {full-ord }}$ denote the preimage of $\left(\overrightarrow{\mathrm{M}}_{\mathcal{H}}^{\min } \otimes_{\mathbb{Z}} \mathbb{F}_{p}\right)^{\text {full-ord }}$ under (7.12). By the argument of [31, Sections 6.3.1 and 6.3.2], $\left(\overrightarrow{\mathrm{M}}_{\mathcal{H}, \Sigma}^{\text {tor }}\right)^{\text {full-ord }}$ is also the locus where the pullback $\vec{G}_{j_{0} \text {,aux }}$ of the

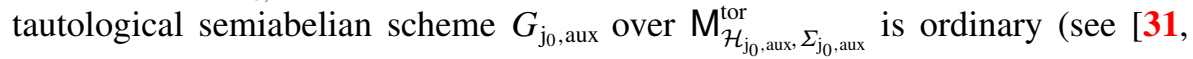
Definition 3.1.1.2]).

Let $\left(Z_{\mathcal{H}}, \Phi_{\mathcal{H}}, \delta_{\mathcal{H}}\right)$ be a representative of cusp label such that $Z_{\mathcal{H}}$ is the $\mathcal{H}$-orbit of some $Z$ such that $\mathrm{Gr}_{-1}^{\mathrm{Z}}=\{0\}$ as in the statement of the proposition. By Theorem 10.13, for any $\sigma \in \Sigma_{\Phi_{\mathcal{H}}}$ such that $\sigma \subset \mathbf{P}_{\Phi_{\mathcal{H}}}^{+}$, since (10.3) is induced by (8.33) (whose composition with (7.8) is (8.32)), the pullback of $\vec{G}_{\mathrm{j}_{0} \text {,aux }}$ to the $\left[\left(\Phi_{\mathcal{H}}, \delta_{\mathcal{H}}, \sigma\right)\right]$-stratum $\vec{Z}_{\left[\left(\Phi_{\mathcal{H}}, \delta_{\mathcal{H}}, \sigma\right)\right]}$ of $\overrightarrow{\mathrm{M}}_{\mathcal{H}, \Sigma}^{\text {tor }}$ is a split torus with character group $X_{\mathrm{j}_{0} \text {, aux }}$, which is ordinary by definition. So $\overrightarrow{\mathrm{Z}}_{\left[\left(\Phi_{\mathcal{H}}, \delta_{\mathcal{H}}, \sigma\right)\right]} \otimes_{\mathbb{Z}} \mathbb{F}_{p} \subset\left(\overrightarrow{\mathrm{M}}_{\mathcal{H}, \Sigma}^{\text {tor }} \otimes_{\mathbb{Z}} \mathbb{F}_{p}\right)^{\text {full-ord }}$, and therefore $\vec{Z}_{\left[\left(\Phi_{\mathcal{H}}, \delta_{\mathcal{H}}\right)\right]} \otimes_{\mathbb{Z}} \mathbb{F}_{p} \subset\left(\overrightarrow{\mathrm{M}}_{\mathcal{H}}^{\min } \otimes_{\mathbb{Z}} \mathbb{F}_{p}\right)^{\text {full-ord }}$ (by Theorem 12.1) (this is essentially the same argument as in the proof of [31, Corollary 6.3.3.2]). Thus, in order to show that $\left(\overrightarrow{\mathrm{M}}_{\mathcal{H}}^{\min } \otimes_{\mathbb{Z}} \mathbb{F}_{p}\right)^{\text {full-ord }}$ is dense in $\overrightarrow{\mathrm{M}}_{\mathcal{H}}^{\text {min }} \otimes_{\mathbb{Z}} \mathbb{F}_{p}$, it suffices to show that every irreducible component of $\vec{M}_{\mathcal{H}}^{\text {min }} \otimes_{\mathbb{Z}} \overline{\mathbb{F}}_{p}$ has a nonempty intersection with $\vec{Z}_{\left[\left(\Phi_{\mathcal{H}}, \delta_{\mathcal{H}}\right)\right]} \otimes_{\mathbb{Z}} \overline{\mathbb{F}}_{p}$ for some $\left(Z_{\mathcal{H}}, \Phi_{\mathcal{H}}, \delta_{\mathcal{H}}\right)$ as above. Since $\vec{Z}_{\left[\left(\Phi_{\mathcal{H}}, \delta_{\mathcal{H}}\right)\right]}$ is closed in $\overrightarrow{\mathrm{M}}_{\mathcal{H}}^{\min }$ because $\mathrm{Gr}_{-1}^{\mathrm{Z}}=\{0\}$ (see Theorem 12.1), by Corollary 14.4, it suffices to show that each irreducible component of $\mathrm{M}_{\mathcal{H}}^{\min } \otimes_{\mathbb{Z}} \mathbb{C} \cong \overrightarrow{\mathrm{M}}_{\mathcal{H}}^{\min } \otimes_{\mathbb{Z}} \mathbb{C}$ has a nonempty intersection with $Z_{\left[\left(\Phi_{\mathcal{H}}, \delta_{\mathcal{H}}\right)\right]} \otimes_{\mathbb{Z}} \mathbb{C} \cong \vec{Z}_{\left[\left(\Phi_{\mathcal{H}}, \delta_{\mathcal{H}}\right)\right]} \otimes_{\mathbb{Z}} \mathbb{C}$ for some $\left(\mathrm{Z}_{\mathcal{H}}, \Phi_{\mathcal{H}}, \delta_{\mathcal{H}}\right)$ as above.

Since $\mathcal{O} \otimes_{\mathbb{Z}} \mathbb{Q}$ involves no simple factor of type $\mathrm{D}$, and since the condition $\mathrm{Gr}_{-1}^{\mathrm{Z}}=\{0\}$ forces the rank of each $\mathbb{Q}$-simple factor of $L$ to be even, it follows from the calculation in [26, Section 8, page 400] that the so-called failure of Hasse's principle does not occur for $\mathrm{M}_{\mathcal{H}}$. Hence every Z such that $\mathrm{Gr}_{-1}^{\mathrm{Z}}=\{0\}$ must come from some filtration $V$ of $L \otimes_{\mathbb{Z}} \mathbb{Q}$ as in [31, Lemma 1.2.3.1 and (1.2.3.2)] such that $\mathrm{Gr}_{-1}^{\mathrm{V}}=\{0\}$. This implies the desired assertion in the previous paragraph by comparison with the complex analytic construction in [3, Theorem 10.11] and [49, Section 6.2], as in [28, Theorem 5.1.1].

COROLlaRY 15.2. Suppose we are in the setting of either of Remark 14.5, Lemma 14.6, or Lemma 14.7. In the setting of Remark 14.5, we assume moreover that $p$ splits completely in the reflex field $F_{0}$ defined by the integral PEL datum $\left(\mathcal{O}, \star, L,\langle\cdot, \cdot\rangle, h_{0}\right)$ as in [30, Definition 1.2.5.4]. Then $\left(\overrightarrow{\mathrm{M}}_{\mathcal{H}}^{\min } \otimes_{\mathbb{Z}} \mathbb{F}_{p}\right)^{\text {full-ord }}$ and $\left(\overrightarrow{\mathrm{M}}_{\mathcal{H}} \otimes_{\mathbb{Z}} \mathbb{F}_{p}\right)^{\text {full-ord }}$ are nonempty and dense in $\overrightarrow{\mathrm{M}}_{\mathcal{H}}^{\mathrm{min}} \otimes_{\mathbb{Z}} \mathbb{F}_{p}$ and $\overrightarrow{\mathrm{M}}_{\mathcal{H}} \otimes_{\mathbb{Z}} \mathbb{F}_{p}$, respectively. 
Proof. In the setting of either of Remark 14.5, Lemma 14.6, or Lemma 14.7, we have $\mathcal{H}=\mathcal{H}^{p} \mathrm{G}\left(\mathbb{Z}_{p}\right)$ for some neat open compact subgroup $\mathcal{H}^{p}$ of $\mathrm{G}\left(\hat{\mathbb{Z}}^{p}\right)$ (nevertheless, as explained in the proof of Proposition 15.1, the cases of other levels can be reduced to this case). If $p$ is a good prime as in Remark 14.5, then it follows from [60] (as explained in the beginning of [31, Section 6.3.3]) that $\left(\overrightarrow{\mathrm{M}}_{\mathcal{H}} \otimes_{\mathbb{Z}} \mathbb{F}_{p}\right)^{\text {full-ord }}$ is nonempty and dense in $\overrightarrow{\mathrm{M}}_{\mathcal{H}} \otimes_{\mathbb{Z}} \mathbb{F}_{p}$, and that $\left(\overrightarrow{\mathrm{M}}_{\mathcal{H}}^{\min } \otimes_{\mathbb{Z}} \mathbb{F}_{p}\right)^{\text {full-ord }}$ is also dense in $\overrightarrow{\mathrm{M}}_{\mathcal{H}}^{\min } \otimes_{\mathbb{Z}} \mathbb{F}_{p}$, because $\overrightarrow{\mathrm{M}}_{\mathcal{H}} \otimes_{\mathbb{Z}} \mathbb{F}_{p}$ is dense in $\overrightarrow{\mathrm{M}}_{\mathcal{H}}^{\text {min }} \otimes_{\mathbb{Z}} \mathbb{F}_{p}$ by Corollary 12.5. In the setting of either of Lemma 14.6 or Lemma 14.7, the desired assertions follow from Proposition 15.1.

REMARK 15.3. When \#J $>1$, we do not expect $\left(\overrightarrow{\mathrm{M}}_{\mathcal{H}} \otimes_{\mathbb{Z}} \mathbb{F}_{p}\right)^{\text {full-ord }}$ to be dense in $\overrightarrow{\mathrm{M}}_{\mathcal{H}} \otimes_{\mathbb{Z}} \mathbb{F}_{p}$ in general. See [54] and [20, Corollary 3.11.3 and Section 3.12] for examples where the ordinary loci are nonempty but not dense.

\section{Concluding remarks}

Let us compare the results obtained in this article with the main results in [30] in the good reduction case (we shall not compare our results in this article with those in works earlier than [30]. See the introduction of [30] for an indirect comparison).

REMARK 16.1. Compared with [30, Theorem 6.4.1.1], which is the main result on integral models of toroidal compactifications in the good reduction case, the results obtained in this article achieved the following.

(1) For sufficiently many compatible choices $\Sigma$ of admissible (possibly nonsmooth) rational polyhedral cone decompositions allowing the consideration of Hecke actions (in a somewhat subtle sense; see Examples 2.5 and 13.14, and Proposition 13.15), we can define the toroidal compactifications $\overrightarrow{\mathrm{M}}_{\mathcal{H}, \Sigma}^{\text {tor }}$ of $\overrightarrow{\mathrm{M}}_{\mathcal{H}}$, which carry a collection of degeneration families $\left(\vec{G}_{\mathrm{j}}, \vec{\lambda}_{\mathrm{j}}, \vec{i}_{\mathrm{j}}, \vec{\alpha}_{\mathcal{H}_{\mathrm{j}}}\right)$ of types $\mathrm{M}_{\mathcal{H}_{\mathrm{j}}}$, for all $\mathrm{j} \in \mathrm{J}$, extending the tautological tuples over $\mathrm{M}_{\mathcal{H}_{\mathrm{j}}} \cong \mathrm{M}_{\mathcal{H}}$, by Theorem 11.2. This generalizes [30, Theorem 6.4.1.1(1)].

(2) The scheme $\overrightarrow{\mathrm{M}}_{\mathcal{H}, \Sigma}^{\text {tor }}$ is proper but certainly not smooth in general. By Proposition 14.2, the local property of $\overrightarrow{\mathrm{M}}_{\mathcal{H}, \Sigma}^{\text {tor }}$ is as nice as the one of $\overrightarrow{\mathrm{M}}_{\mathcal{H}}$, in terms of normality (by construction), geometric normality of fibers, and Cohen-Macaulayness (or more generally the properties of fibers considered there). 
(3) The stratification we obtained in Theorem 9.13 generalizes [30, Theorem 6.4.1.1(2)]. Since the strata are generally not smooth over $\overrightarrow{\mathrm{S}}_{0}$, there are some subtleties that needs to be-and can be-addressed. For example, by Corollary 10.15 , each stratum is flat over $\overrightarrow{\mathrm{S}}_{0}$ and normal; by Corollary 10.18 , $\overrightarrow{\mathrm{M}}_{\mathcal{H}} \otimes_{\mathbb{Z}} \mathbb{F}_{p}$ is open and dense in $\overrightarrow{\mathrm{M}}_{\mathcal{H}, \Sigma}^{\text {tor }} \otimes_{\mathbb{Z}} \mathbb{F}_{p}$.

(4) While we cannot assert that the boundary $\overrightarrow{\mathrm{M}}_{\mathcal{H}, \Sigma}^{\text {tor }}-\overrightarrow{\mathrm{M}}_{\mathcal{H}}$ (with its reduced structure) is a normal crossing divisor as in [30, Theorem 6.4.1.1(3)], the formal local description along the boundary strata will still be given by the analog of [30, Theorem 6.4.1.1(5)] below (one can also introduce the language of log structures, but we have not spelled that out).

(5) We do not have an analog of [30, Theorem 6.4.1.1(4)] because the extended Kodaira-Spencer morphism is generally undefined in our context.

(6) The comparison of formal completions we obtained in Theorem 10.13 generalizes [30, Theorem 6.4.1.1(5)].

(7) The universal property of $\overrightarrow{\mathrm{M}}_{\mathcal{H}, \Sigma}^{\text {tor }}$ we obtained in Theorems 7.14 and 11.4 generalizes [30, Theorem 6.4.1.1(6)].

(8) While the very construction of $\overrightarrow{\mathrm{M}}_{\mathcal{H}, \Sigma}^{\text {tor }}$ in Section 7 depends on the auxiliary choices in Sections 4 and 5, by Corollary 11.7, up to canonical isomorphism, the resulted $\overrightarrow{\mathrm{M}}_{\mathcal{H}, \Sigma}^{\text {tor }}$ is in fact independent of the auxiliary choices.

REMARK 16.2. Compared with [30, Theorem 7.2.4.1], which is the main result on integral models of minimal compactifications in the good reduction case, the results obtained in this article achieved the following.

(1) Essentially by construction, the scheme $\overrightarrow{\mathrm{M}}_{\mathcal{H}}^{\text {min }}$ is projective and flat over $\overrightarrow{\mathrm{S}}_{0}:=\operatorname{Spec}\left(\mathcal{O}_{F_{0},(p)}\right)$, and $\overrightarrow{\mathrm{M}}_{\mathcal{H}}^{\text {min }}$ contains $\overrightarrow{\mathrm{M}}_{\mathcal{H}}$ as an open dense subscheme. This generalizes [30, Theorem 7.2.4.1(1)].

(2) Also essentially by construction, some power of the Hodge invertible sheaf $\omega_{\mathrm{M}_{\mathcal{H}}}$ over $\mathrm{M}_{\mathcal{H}}$ extends to an ample invertible sheaf $\omega_{\overrightarrow{\mathrm{M}}_{\mathcal{H}}^{\mathrm{min}}}$ over $\overrightarrow{\mathrm{M}}_{\mathcal{H}}^{\min }$. This generalizes [30, Theorem 7.2.4.1(2)].

(3) The assertion in Proposition 7.11 that $\overrightarrow{\mathrm{M}}_{\mathcal{H}}^{\min } \cong \operatorname{Proj}\left(\bigoplus_{k \geqslant 0} \Gamma\left(\overrightarrow{\mathrm{M}}_{\mathcal{H}, \Sigma}^{\text {tor }}\right.\right.$, $\left.\left.\omega_{\overrightarrow{\mathrm{M}}_{\mathcal{H}, \Sigma}^{\text {tor }}, \mathrm{J}}^{\otimes k}\right)\right)$ generalizes part of [30, Theorem 7.2.4.1(3)]. 
(4) The stratification we obtained in Theorems 12.1 and 12.16 generalizes [30, Theorem 7.2.4.1 (4) and (5)]. By Corollary 12.5 (see also Remark 12.6), $\overrightarrow{\mathrm{M}}_{\mathcal{H}} \otimes_{\mathbb{Z}} \mathbb{F}_{p}$ is open and dense in $\overrightarrow{\mathrm{M}}_{\mathcal{H}}^{\min } \otimes_{\mathbb{Z}} \mathbb{F}_{p}$.

(5) While the very construction of $\overrightarrow{\mathrm{M}}_{\mathcal{H}}^{\min }$ in Proposition 7.11 depends on the auxiliary choices in Sections 4 and 5, by Corollary 12.7, up to canonical isomorphism, the resulted $\overrightarrow{\mathrm{M}}_{\mathcal{H}}^{\min }$ is in fact independent of the auxiliary choices.

Thus, perhaps surprisingly, many features of the 'good reduction' theory in [30] extend to the 'bad reduction' theory for constructions by normalization, regardless of the ramification, levels, and polarization degrees involved (nevertheless, for this to be useful, the input from the theory of local models is often crucial, as we have seen in the examples in Sections 14 and 15. See Remarks 16.4 and 16.5 below).

REMARK 16.3. (This remark was updated after we received the latest revision of [34] on February 25, 2015.) The same constructions by taking normalizations of good reduction auxiliary models have been considered in [34] for general Hodge-type Shimura varieties, and results similar to ours have been obtained under the additional assumption that the level $\mathcal{H}$ is exactly the preimage of $\prod_{\mathrm{j} \in \mathrm{J}} \mathcal{H}_{\mathrm{j} \text {,aux }}$ under the homomorphism $\mathrm{G}\left(\mathbb{A}^{\infty}\right) \rightarrow \prod_{\mathrm{j} \in \mathrm{J}} \mathrm{G}_{\mathrm{j} \text {,aux }}\left(\mathbb{A}^{\infty}\right)$ induced by (4.10), using a rather different method based on rationality properties of Hodge tensors. Nevertheless, our methods are closer to those in [10] and [30], and hence are logically simpler (because the Hodge-type methods in [34] also depend on [10] and [30]). Moreover, we allow the level $\mathcal{H}$ to be arbitrarily high at $p$, and we have shown that all the geometric objects constructed by normalization are independent of the auxiliary choices (such independence seems rather subtleand perhaps not meaningful-in general Hodge-type cases). In these regards, our results are not yet subsumed by the latest version of [34], and the semiabelian degenerations parameterized by our integral models of toroidal compactifications might be useful in certain applications.

REMARK 16.4. The toroidal and minimal compactifications constructed in [55] and [56] are for the Siegel moduli with parahoric levels at $p$ defined by linear algebraic data that are otherwise split, in which case the naive moduli problems as in Example 13.12 are not naive and define good integral models. The constructions rely crucially on the assertion that the integral models (before compactification) are normal, which is shown there using results of [44] and [12]. The Siegel moduli with pro- $p$-Iwahoric levels at $p$ have also been considered in [57]. Again, the constructions crucially rely on the assertion that the 
integral models before compactification are normal, which is shown there using results of [44], [12], and [21]. In fact, these integral models with Iwahoric and pro- $p$-Iwahoric levels at $p$ have been shown to be normal and Cohen-Macaulay. If we use the constructions in this article instead, then we obtain the same (projective normal) minimal compactifications as in [56] and [57], and sufficiently many (but not all) normal and Cohen-Macaulay toroidal compactifications as in [55] and [57], which admit stratifications and formal local descriptions compatible with those in [10] and [30] in characteristic zero.

REMARK 16.5. Local models for moduli problems of abelian schemes with PEL structures at parahoric levels at $p$ (as in Examples 2.4 and 13.12) have been extensively studied in the last two decades (see the survey articles [50], [16], and [48], and see [62] and [47] for some important recent developments). In all cases where the local models are known to be flat and normal, our constructions give toroidal and minimal compactifications for them, with local properties of the toroidal compactifications such as the normality of geometric fibers and Cohen-Macaulayness (or more generally the properties of fibers considered in Proposition 14.2) as nice as the integral models before compactification, and with stratifications and formal local descriptions compatible with those in [10] and [30] in characteristic zero.

REMARK 16.6. It remains unclear what one can really say about substantially higher levels. The simple-minded but indirect constructions by normalization do produce reasonably good models for arbitrary levels, as we have shown in this article. But for many applications one will still need to relate them to some more meaningful or direct constructions. We do not have a good strategy at this moment.

\section{Acknowledgments}

The author was partially supported by the National Science Foundation under agreement No. DMS-1352216, and by an Alfred P. Sloan Research Fellowship. He would like to thank Keerthi Madapusi Pera, George Pappas, and Xinwen Zhu for very helpful discussions, and the anonymous referee for many thoughtful suggestions.

\section{References}

[1] J. Arthur, D. Ellwood and R. Kottwitz (Eds.), Harmonic Analysis, the Trace Formula, and Shimura Varieties. Proceedings of the Clay Mathematics Institute 2003 Summer School, 
The Fields Institute, Toronto, Canada, June 2-27, 2003, Clay Mathematics Proceedings, 4 (American Mathematical Society, Providence, Rhode Island, 2005), Clay Mathematics Institute, Cambridge, MA.

[2] A. Ash, D. Mumford, M. Rapoport and Y. Tai, Smooth Compactification of Locally Symmetric Varieties, Lie Groups: History Frontiers and Applications, 4 (Mathematical Science Press, Brookline, MA, 1975).

[3] W. L. Baily Jr. and A. Borel, 'Compactification of arithmetic quotients of bounded symmetric domains', Ann. of Math. (2) 84(3) (1966), 442-528.

[4] S. Bosch, W. Lütkebohmert and M. Raybaud, Néron Models, Ergebnisse der Mathematik und ihrer Grenzgebiete, 3. Folge, 21 (Springer, Berlin, Heidelberg, New York, 1990).

[5] J.-B. Bost, P. Boyer, A. Genestier, L. Lafforgue, S. Lysenko, S. Morel and B. C. Ngô (Eds.), De la Géometrie Algébrique aux Formes Automorphes (II): Une collection d'articles en l'honneur du soixantième Anniversaire de Gérard Laumon, Astérisque, 370 (Société Mathématique de France, Paris, 2015).

[6] A. J. de Jong, 'The moduli spaces of polarized abelian varieties', Math. Ann. 295 (1993), 485-503.

[7] P. Deligne and W. Kuyk (Eds.), Modular Functions of One Variable II, Lecture Notes in Mathematics, 349 (Springer, Berlin, Heidelberg, New York, 1973).

[8] P. Deligne and D. Mumford, 'The irreducibility of the space of curves of given genus', Publ. Math. Inst. Hautes Études Sci. 36 (1969), 75-109.

[9] P. Deligne and M. Rapoport, Les schémas de modules de courbes elliptiques, in Deligne and Kuyk [7], 143-316.

[10] G. Faltings and C.-L. Chai, Degeneration of Abelian Varieties, Ergebnisse der Mathematik und ihrer Grenzgebiete, 3. Folge, 22 (Springer, Berlin, Heidelberg, New York, 1990).

[11] G. Farkas and I. Morrison (Eds.), Handbook of Moduli: Volume III, Advanced Lectures in Mathematics, 24 (International Press, Somerville, MA, 2013), Higher Education Press, Beijing.

[12] U. Görtz, 'On the flatness of local models for the symplectic group', Adv. Math. 176 (2003), 89-115.

[13] A. Grothendieck (Ed.), Revêtements Étales et Groupe Fondamental (SGA 1), Lecture Notes in Mathematics, 224 (Springer, Berlin, Heidelberg, New York, 1971).

[14] A. Grothendieck and J. Dieudonné, Eléments de Géométrie Algébrique, Publications Mathématiques de Institut des Hautes Etudes Scientifiques, 4, 8, 11, 17, 20, 24, 28, 32 (Institut des Hautes Etudes Scientifiques, Paris, 1960, 1961, 1963, 1964, 1965, 1966, 1967).

[15] A. Grothendieck and J. Dieudonné, Eléments de Géométrie Algébrique I: Le Langage des Schémas, Grundlehren der Mathematischen Wissenschaften, 166 (Springer, Berlin, Heidelberg, New York, 1971).

[16] T. J. Haines, Introduction to Shimura varieties with bad reductions of parahoric type, in Arthur et al. [1], 583-658.

[17] M. Hakim, Topos Annelés et Schémas Relatifs, Ergebnisse der Mathematik und ihrer Grenzgebiete, 64 (Springer, Berlin, Heidelberg, New York, 1972).

[18] M. Harris, 'Functorial properties of toroidal compactifications of locally symmetric varieties', Proc. Lond. Math. Soc. (3) 59 (1989), 1-22.

[19] M. Harris, K.-W. Lan, R. Taylor and J. Thorne, On the rigid cohomology of certain Shimura varieties, Preprint, 2013. 
[20] P. Hartwig, Kottwitz-Rapoport and $p$-rank strata in the reduction of Shimura varieties of PEL type, Preprint, 2012.

[21] X. He, 'Normality and Cohen-Macaulayness of local models of Shimura varieties', Duke Math. J. 162(13) (2013), 2509-2523.

[22] M. Hochster, 'Rings of invariants of tori, Cohen-Macaulay rings generated by monomials, and polytopes', Ann. of Math. (2) 96(2) (1972), 318-337.

[23] K. Kato, 'Toric singularities', Amer. J. Math. 116(5) (1994), 1073-1099.

[24] G. Kempf, F. Knudsen, D. Mumford and B. Saint-Donat, Toroidal Embeddings I, Lecture Notes in Mathematics, 339 (Springer, Berlin, Heidelberg, New York, 1973).

[25] M. Kisin, 'Integral models for Shimura varieties of abelian type', J. Amer. Math. Soc. 23(4) (2010), 967-1012.

[26] R. E. Kottwitz, 'Points on some Shimura varieties over finite fields', J. Amer. Math. Soc. 5(2) (1992), 373-444.

[27] K.-W. Lan, 'Elevators for degenerations of PEL structures', Math. Res. Lett. 18(5) (2011), 889-907.

[28] K.-W. Lan, 'Comparison between analytic and algebraic constructions of toroidal compactifications of PEL-type Shimura varieties', J. Reine Angew. Math. 664 (2012), 163-228. errata available online at the author's website.

[29] K.-W. Lan, 'Toroidal compactifications of PEL-type Kuga families', Algebra Number Theory 6(5) (2012), 885-966. errata available online at the author's website.

[30] K.-W. Lan, Arithmetic Compactification of PEL-type Shimura Varieties, London Mathematical Society Monographs, 36 (Princeton University Press, Princeton, 2013), errata and revision available online at the author's website.

[31] K.-W. Lan, Compactifications of PEL-type Shimura varieties and Kuga families with ordinary loci, Preprint, 2013.

[32] R. P. Langlands and D. Ramakrishnan (Eds.), The Zeta Functions of Picard Modular Surfaces: Based on Lectures Delivered at a CRM Workshop in the Spring of 1988, (Les Publications CRM, Montréal, 1992).

[33] Q. Liu, Algebraic Geometry and Arithmetic Curves, Oxford Graduate Texts in Mathematics, 6 (Oxford University Press, Oxford, 2002), translated by Reinie Erné.

[34] K. Madapusi Pera, Toroidal compactifications of integral models of Shimura varieties of Hodge type, Preprint, 2015.

[35] H. Matsumura, Commutative Algebra, 2nd edn, Mathematics Lecture Note Series (The Benjamin/Cummings Publishing Company, Inc., 1980).

[36] H. Matsumura, Commutative Ring Theory, Cambridge Studies in Advanced Mathematics, 8 (Cambridge University Press, Cambridge, New York, 1986).

[37] J. S. Milne, The points on a Shimura variety modulo a prime of good reduction, in Langlands and Ramakrishnan [32], 151-253.

[38] B. Moonen, 'Models of Shimura varieties in mixed characteristic', in Galois Representations in Arithmetic Algebraic Geometry, [53], 267-350.

[39] L. Moret-Bailly, Pinceaux de Variétés Abéliennes, Astérisque, 129 (Société Mathématique de France, Paris, 1985).

[40] L. Moret-Bailly, 'Un problème de descente', Bull. Soc. Math. France 124 (1996), 559-585.

[41] D. Mumford, Abelian Varieties, Tata Institute of Fundamental Research Studies in Mathematics, 5 (Oxford University Press, Oxford, 1970), with appendices by C. P. Ramanujam and Y. Manin.

[42] D. Mumford, 'An analytic construction of degenerating abelian varieties over complete rings', Compos. Math. 24(3) (1972), 239-272. 
[43] D. Mumford, J. Fogarty and F. Kirwan, Geometric Invariant Theory, 3rd edn, Ergebnisse der Mathematik und ihrer Grenzgebiete, 34 (Springer, Berlin, Heidelberg, New York, 1994).

[44] B. C. Ngô and A. Genestier, 'Alcôves et p-rang des variétés abéliennes', Ann. Inst. Fourier. Grenoble 52(6) (2002), 1665-1680.

[45] G. Pappas and M. Rapoport, 'Local models in the ramified case, I. The EL-case', J. Algebraic Geom. 12(1) (2003), 107-145.

[46] G. Pappas and M. Rapoport, 'Local models in the ramified case, II. Splitting models', Duke Math. J. 127(2) (2005), 193-250.

[47] G. Pappas and X. Zhu, 'Local models of Shimura varieties and a conjecture of Kottwitz', Invent. Math. 194 (2013), 147-254.

[48] G. Pappas, M. Rapoport and B. Smithling, 'Local models of Shimura varieties, I. Geometry and combinatorics', in Farkas and Morrison [11], 135-217.

[49] R. Pink, 'Arithmetic compactification of mixed Shimura varieties', PhD Thesis, Rheinischen Friedrich-Wilhelms-Universität, Bonn, 1989.

[50] M. Rapoport, 'A guide to the reduction modulo $p$ of Shimura varieties', in Tilouine et al. [58], 271-318.

[51] M. Rapoport and T. Zink, Period Spaces for p-Divisible Groups, Annals of Mathematics Studies, 141 (Princeton University Press, Princeton, 1996).

[52] M. Raynaud, Faisceaux amples sur les schémas en groupes et les espaces homogènes, Lecture Notes in Mathematics, 119 (Springer, Berlin, Heidelberg, New York, 1970).

[53] A. J. Scholl and R. L. Taylor, Galois Representations in Arithmetic Algebraic Geometry, London Mathematical Society Lecture Note Series, 254 (Cambridge University Press, Cambridge, New York, 1998).

[54] H. Stamm, 'On the reduction of the Hilbert-Blumenthal-moduli scheme with $\Gamma_{0}(p)$-level structure', Forum Math. 9(4) (1997), 405-455.

[55] B. Stroh, 'Compactification de variétés de Siegel aux places de mauvaise réduction', Bull. Soc. Math. France 138(2) (2010), 259-315.

[56] B. Stroh, 'Compactification minimale et mauvaise réduction', Ann. Inst. Fourier. Grenoble 60(3) (2010), 1035-1055.

[57] B. Stroh, 'Mauvaise réduction au bord', in Bost et al. [5], 269-304.

[58] J. Tilouine, H. Carayol, M. Harris and M.-F. Vignéras (Eds.), Formes automorphes (I): actes du Semestre du Centre Émile Borel, printemps 2000, Astérisque, 298 (Société Mathématique de France, Paris, 2005).

[59] A. Vasiu, 'Integral canonical models of Shimura varieties of preabelian type', Asian J. Math. 3 (1999), 401-518.

[60] T. Wedhorn, 'Ordinariness in good reductions of Shimura varieties of PEL-type', Ann. Sci. Éc. Norm. Supér. (4) 32 (1999), 575-618.

[61] Y. G. Zarhin, 'Endomorphisms of abelian varieties and points of finite order in characteristic p', Math. Notes 21(6) (1977), 415-419.

[62] X. Zhu, 'On the coherence conjecture of Pappas and Rapoport', Ann. of Math. (2) 180(1) (2014), 1-85. 